\title{
The elephant in the room: What matters cognitively in cumulative technological culture
}

\section{François Osiurak ${ }^{\mathrm{a}, \mathrm{b}} \odot$ and Emanuelle Reynaud ${ }^{\mathrm{a}}$}

${ }^{a}$ Department of Psychology, University of Lyon, 69007 Lyon, France and ${ }^{\mathrm{b}}$ French University Institute, 75231 Paris, France.

francois.osiurak@univ-lyon2.fr

https://emc.univ-lyon2.fr/fr/equipes/equipe-cognition-outils-systemes/francois-osiurak/francois-osiurak-610797.kjsp emanuelle.reynaud@univ-lyon2.fr

https://emc.univ-lyon2.fr/fr/equipes/equipe-cognition-outils-systemes/emanuelle-reynaud/

\section{Abstract}

Cumulative technological culture (CTC) refers to the increase in the efficiency and complexity of tools and techniques in human populations over generations. A fascinating question is to understand the cognitive origins of this phenomenon. Because CTC is definitely a social phenomenon, most accounts have suggested a series of cognitive mechanisms oriented toward the social dimension (e.g., teaching, imitation, theory of mind, and metacognition), thereby minimizing the technical dimension and the potential influence of non-social, cognitive skills. What if we have failed to see the elephant in the room? What if social cognitive mechanisms were only catalyzing factors and not the sufficient and necessary conditions for the emergence of CTC? In this article, we offer an alternative, unified cognitive approach to this phenomenon by assuming that CTC originates in non-social cognitive skills, namely technical-reasoning skills which enable humans to develop the technical potential necessary to constantly acquire and improve technical information. This leads us to discuss how theory of mind and metacognition, in concert with technical reasoning, can help boost CTC. The cognitive approach developed here opens up promising new avenues for reinterpreting classical issues (e.g., innovation, emulation vs. imitation, social vs. asocial learning, cooperation, teaching, and overimitation) in a field that has so far been largely dominated by other disciplines, such as evolutionary biology, mathematics, anthropology, archeology, economics, and philosophy.

\section{Introduction}

The term cumulative technological culture (CTC) is used to describe how, over time, human populations have gradually accumulated techniques and tools that are too complex to be invented by a single individual (Boyd \& Richerson 1996; Boyd et al. 2011; Richerson \& Boyd 2005; Tomasello 1999; Tomasello et al. 1993), a phenomenon that could be restricted to humans (Boyd \& Richerson 1996; Galef 1992; Tomasello 1999; but see Boesch \& Tomasello 1998; Hunt \& Gray 2003; Whiten et al. 2003). CTC has been said to be driven by two engines (Legare \& Nielsen 2015): imitation (faithful copying of a trait) and innovation (improvement of a trait). ${ }^{1}$ To date, the emphasis has been placed mainly on the imitative component (Boyd \& Richerson 1985; Dean et al. 2012; Derex et al. 2013b; Galef 1992; Henrich \& Gil-White 2001; Lewis \& Laland 2012; Schillinger et al. 2015; Tennie et al. 2009; 2012; Tomasello 1999; Tomasello et al. 1993; 2005). The rationale is that faithful social transmission can work as a ratchet to prevent slippage backward so that the newly invented technique can be subsequently improved (i.e., ratchet effect; Tomasello 1999; Tomasello et al. 1993). In line with this, the literature has suggested a series of cognitive mechanisms that are oriented toward the social dimension of CTC (e.g., teaching, imitation, theory of mind, and metacognition). The emphasis on the social dimension is obviously justified: Social learning ${ }^{2}$ (whatever it may be) is a far more effective catalyst than asocial learning when it comes to passing on the content of technical information possessed by individuals in a group. However, this does not necessarily imply that CTC originates, first and foremost, in social cognitive skills. A viable alternative explanation is that CTC has emerged because of a non-social cognitive structure that allows humans to acquire and develop this content.

To illustrate this content-structure distinction, let us consider the following assumption: "[H]umans may be smarter than other creatures, but none of us is nearly smart enough to acquire all the information necessary to survive in any single habitat [...] We owe our success to our uniquely developed ability to learn from others" (Boyd et al. 2011, p. 10918). This assumption emphasizes the social dimension of CTC and how the content can be acquired. Let us propose a revised version of this assumption: None of us is nearly smart enough to acquire all the information necessary to survive in any single habitat, but all of us are smart enough to acquire each piece of information - as well as to produce any kind of 
innovation - necessary to survive in any single habitat. This revised assumption allows us to escape from the social dimension and to focus instead on the non-social, technical dimension.

This assumption has two corollaries. The first is that all humans possess a non-social cognitive structure enabling them to acquire and generate a great - perhaps infinite - amount of technical information (i.e., the content). This is what we call the technical potential. This potential is fundamental for CTC because without it, we would not have observed an almost systematic, and sometimes very fast (e.g., digital technology revolution), increase in accumulated expertise over time. An individual may live in a very rich technical environment (i.e., one with rich content). However, if this individual does not possess the ability (i.e., the necessary cognitive structure) to acquire and improve this content, this rich environment is of no interest and it is unlikely that imitation and innovation will be observed. Our room for technical improvement revealed by CTC is necessarily based on our extensive individual cognitive ability to acquire and improve techniques. The second corollary is that this technical potential is influenced by several factors: non-social environmental opportunities (e.g., seeing lightning striking a tree), social environmental opportunities (e.g., seeing another individual making fire), and idiosyncratic characteristics (e.g., age, personality, and motivation). Thus, even if all humans possess the cognitive skills (i.e., the structure) allowing them to acquire and generate a vast amount of technical information (i.e., the content), the exploitation of this technical potential depends on orthogonal factors (i.e., each individual's specific historical trajectory resulting from non-social and social opportunities as well as idiosyncratic characteristics, see immediately above) and this leads to interindividual variation in terms of technical expertise.

This article aims to lay the foundations for a cognitive approach to CTC. Our working hypothesis is that CTC originates in uniquely human cognitive skills, namely technical-reasoning skills. Technical reasoning is the necessary cognitive structure that enables humans to constantly acquire and develop new techniques (also called mechanical knowledge). In section 2, we will present the theoretical framework of the technical-reasoning

François Osiurak is Professor of Psychology at the University of Lyon, France. He earned his Ph.D at the University of Angers, France, in 2007. He is the author of over 120 scientific publications in psychology and cognitive science. His research addresses the neurocognitive origins of human tool use and cumulative technological culture. His published articles include "Grasping the affordances, understanding the reasoning: Toward a dialectical theory of human tool use" and "Tool use and affordance: Manipulation-based versus reasoning-based approaches" in the journal Psychological Review. He was appointed as Junior Member of the French University Institute in 2014.

Emanuelle Reynaud is an Associate Professor in cognitive science at the University of Lyon, France. She earned her Ph.D at the Institute of Engineering of Grenoble, France, in 2002. She is a computational cognitive neuroscientist, especially interested in the miracle by which the brain represents an infinite number of objects of thought with a finite number of elements. Computational models and brain imaging help her to solve this great puzzle. She loves interdisciplinary and quantitative analyses of behavior and has worked on a wide variety of methods, including functional magnetic resonance imaging, multi-agent simulations, or eye-tracking. hypothesis based on recent advances in neuropsychology and neuroimaging. We will defend the thesis stating that technicalreasoning skills are the ideal underpinning for both imitation and innovation (the so-called dual engines of CTC). Such a thesis represents an epistemological break with the traditional approach, which assumes that imitation and innovation are based on distinct kinds of mechanisms (i.e., social vs. asocial/individual learning, respectively; Boyd et al. 2011; Dean et al. 2012; Hernik \& Csibra 2009; Tennie et al. 2009; Tomasello 1999), as well as with approaches that reject the potential role of causal understanding (i.e., technical reasoning) in CTC (e.g., Boyd et al. 2011; Derex et al. 2019). In sections 3 and 4, we will then discuss the idea that technical reasoning is also necessary to represent one's own and other people's technical skills, leading us to revise the theory-of-mind and metacognition hypotheses of CTC. In these three sections, we will point to promising new avenues for interpreting classical issues related to CTC. To sum up, this article offers a unified cognitive approach to CTC in a field that has so far been largely dominated by other disciplines, such as evolutionary biology, mathematics, anthropology, archeology, economics, and philosophy (Heyes 2018).

\section{Technical reasoning}

\subsection{Theoretical framework}

Humans are not unique in using and making tools. Many other species also show tool behavior (Bentley-Condit \& Smith 2010; Shumaker et al. 2011). For instance, wild chimpanzees can use and learn how to use stones as hammers to crack nuts in a fashion similar to humans (Boesch et al. 2017; 2019). Wild chimpanzees and capuchin monkeys can also use tools efficiently. They can select and transport different tools according to the properties of the task (e.g., heavier stone hammers to open fresh nuts vs. lighter stone hammers for dry nuts; Luncz et al. 2016; see also Boesch \& Boesch 1984; Deblauwe et al. 2006; Schrauf et al. 2008; Sirianni et al. 2015; 2018; Spagnoletti et al. 2011; Visalberghi et al. 2009a; 2009b; 2015). They can plan economical motor actions when performing tool behavior (Liu et al. 2009; for a review, see Visalberghi et al. 2015). In some cases, they can exhibit multifunctional tool use by using the same tool for different tasks (e.g., using sticks as probes to access small preys and also as combs for grooming; Falótico \& Ottoni 2014; see also Mannu \& Ottoni 2009). New Caledonian crows can also make different types of hook-shaped tools to help them capture their prey (Hunt 1996). In broad terms, a high level of cognitive sophistication can be reflected in nonhuman tool behavior.

However, human tool behavior differs from that known to occur in nonhumans in various respects (Osiurak 2017). For instance, unlike most tool-using species, we can use not only simple tools that amplify motor actions of the upper limbs (e.g., a stick to extend reach and a rock to increase pounding force), but also complex tools that transform our motor actions into qualitatively different mechanical actions (e.g., a knife to cut and a pencil to write; Frey 2007). We are also the only species that exploits "natural" forces (e.g., water, wind, and fire; Shumaker et al. 2011) and exhibits prospective diagnostic skills (Povinelli \& Frey 2016) and transfer skills (Martin-Ordas et al. 2008). ${ }^{3}$ These findings indicate that tool use and making - as well as construction behavior - might be based on distinct cognitive processes in nonhumans and humans. Even if nonhumans including tool-users - may exhibit signs of causal understanding 
(Taylor et al. 2009; van Horik \& Emery 2016), they would not be able to understand unobservable causal properties. By contrast, humans can "reinterpret" the world in terms of unobservable, hypothetical entities such as causal forces (Penn \& Povinelli 2007; Penn et al. 2008b). This non-social cognitive structure might allow us to acquire and develop a vast amount of technical information. This is what we call technical reasoning.

The technical-reasoning hypothesis is akin to previous proposals stressing the uniqueness of humans in terms of causal reasoning ${ }^{4}$ as well as the key role of this kind of reasoning in CTC (e.g., Pinker 2010; Vaesen 2012; Wolpert 2003; see also Penn \& Povinelli 2007; Penn et al. 2008b). It nevertheless differs from some of them in positing that technical reasoning is domain-specific and not domain-general (for a similar viewpoint, see Goldenberg 2013; Hegarty 2004; McCloskey 1983). The technical-reasoning hypothesis is at odds with some of the assumptions of the cultural niche hypothesis (e.g., Boyd et al. 2011). Although this latter hypothesis does not ignore the role of causal reasoning in CTC (notably in its innovative component), it posits that this kind of reasoning plays a minimal role, because humans commonly learn technical content from social interactions without understanding it. This hypothesis is based on what we call the omniscient view of technical reasoning (see sect. 2.3). Moreover, the corollary of this is that our technicalreasoning skills are a product of cultural transmission and not a species-specific difference-maker from which CTC originates. By contrast, the technical-reasoning hypothesis proposes a Bayesian approach to how technical content is acquired through experience, and suggests that technical reasoning is critical for the learning of technical content from social interactions as well as for improving this content (see sects. 2.2 and 2.4). In this view, technical reasoning is central to CTC.

Technical reasoning can be defined as the ability to reason about physical object properties (Osiurak 2014; Osiurak \& Badets 2016; 2017; Osiurak et al. 2010; 2017). It involves the left inferior parietal lobe (and particularly the area PF; Reynaud et al. 2016) and possesses five key characteristics (see Fig. 1):

(1) It is both analogical (i.e., transfer of what is learnt from one situation to another; e.g., using a knife to cut a tomato $\rightarrow$ using a saw to cut a wooden board) and causal (i.e., predicting the effects on the environment; e.g., the tomato is cut in half). This is a specific form of reasoning directed toward the physical world, which must not be confounded with other forms of non-verbal analogical reasoning, particularly fluid cognition. Indeed, fluid cognition is useful for the development of logical thought, which does not need a deep understanding of physical constraints (e.g., Raven's progressive matrices test). In addition, dissociations can be found between technical reasoning and fluid cognition not only at a behavioral level (e.g., De Oliveira et al. 2019), but also at a neural level (left inferior parietal lobe/technical reasoning vs. prefrontal cortex/fluid cognition; Blair 2006).

(2) It is based on mechanical knowledge, that is, knowledge about physical principles (intuitive theories or know-how for Pinker 2010; called content above; e.g., the cutting action requires the relative opposition between one thing possessing the properties Sharpness+, Hardness+, and Width- vs. another thing possessing the properties Sharpness-, Hardness-, and Width+; see Osiurak 2014). This knowledge, which is acquired through experience, is non-declarative. Consequently, when we reason about a physical problem, it is difficult for many of us to make explicit what we understand about the physical principle at work (e.g., just think about how it is difficult to describe a cutting action in physical terms; for a similar viewpoint, see Gatewood 1985; Wynn \& Coolidge 2014). In this way, mechanical knowledge must not be confounded with certain types of semantic knowledge, such as functional knowledge (e.g., van Elk et al. 2014) or folk theories (Keller \& Keller 1996). A significant body of neuropsychological evidence has confirmed this dissociation, indicating that the ability to use familiar or novel tools, on the one hand, and functional knowledge, on the other, can be impaired independently of one another (e.g., Bartolo et al. 2007; Goldenberg \& Spatt 2009; Negri et al. 2007; Osiurak et al. 2008; 2009; Silveri \& Ciccarelli 2009; for a discussion, see Osiurak \& Badets 2016). Functional knowledge is also preferentially impaired after damage to the temporal lobes, as in patients with semantic dementia, who can nevertheless have relatively spared tool-use skills because of the integrity of the left inferior parietal lobe (Baumard et al. 2016; Lesourd et al. 2016). In addition, mechanical knowledge is long-term knowledge that supports technical-reasoning skills and which, if present in sufficient quantity, allow humans to progressively develop technical expertise. In a way, any human is an apprentice or a potential expert-in-the-making, "with bits of knowing accumulating inexorably" (Wynn et al. 2017, p. 47). This view is in line with models of expert cognition that stress the key role of long-term memory in expertise (Ericsson \& Delaney 1999; Ericsson \& Kintsch 1995; Gobet 2016; for a discussion about the link between expert and technical cognition, see Wynn \& Coolidge 2014; Wynn et al. 2017).

(3) The outcome of technical reasoning is a mental simulation of the mechanical action to be performed and this simulation needs to be temporarily maintained to guide the overtly performed action. Technical reasoning allows humans to solve physical problems in everyday life (e.g., to cut a tomato; see Fig. 1). It starts by using mechanical knowledge to find a physical principle that is thought to be (see sect. 2.3) appropriate for solving the targeted physical problem (e.g., cutting) as well as the associated physical object properties (i.e., sharp, and so on). This abstract principle is then transposed to the present situation, which is constrained by certain elements (i.e., the physical properties of the tomato). The individual therefore needs to select a tool that is sharp enough relative to the tomato. During this step, the reasoning ends with the generation of a mental simulation of the mechanical action to be performed (i.e., the motion of the knife on the tomato).

(4) Technical reasoning concerns mechanical actions (i.e., toolobject relationships) and non-motor actions (i.e., hand-tool relationships). Technical reasoning does not deal with the selection and on-line control of the most appropriate motor actions to realize the mentally generated mechanical action. This is the role of the motor-control system, which involves more superior structures of the parietal lobes (e.g., intraparietal sulci; see Osiurak \& Badets 2017; Reynaud et al. 2016; for a neurocognitive framework of how technical reasoning and the motor-control system can interact, see also Orban \& Caruana 2014; Fig. 1). Importantly, the motor-control system is unaware of the goal of the action (i.e., tool use, tool making, or object transport). If someone has the idea of performing back-and-forth movements with the knife on the tomato, this is the expected effect, which will constrain the motor actions chosen within the motor-control system. Similarly, if someone intends to move an object from one location to 

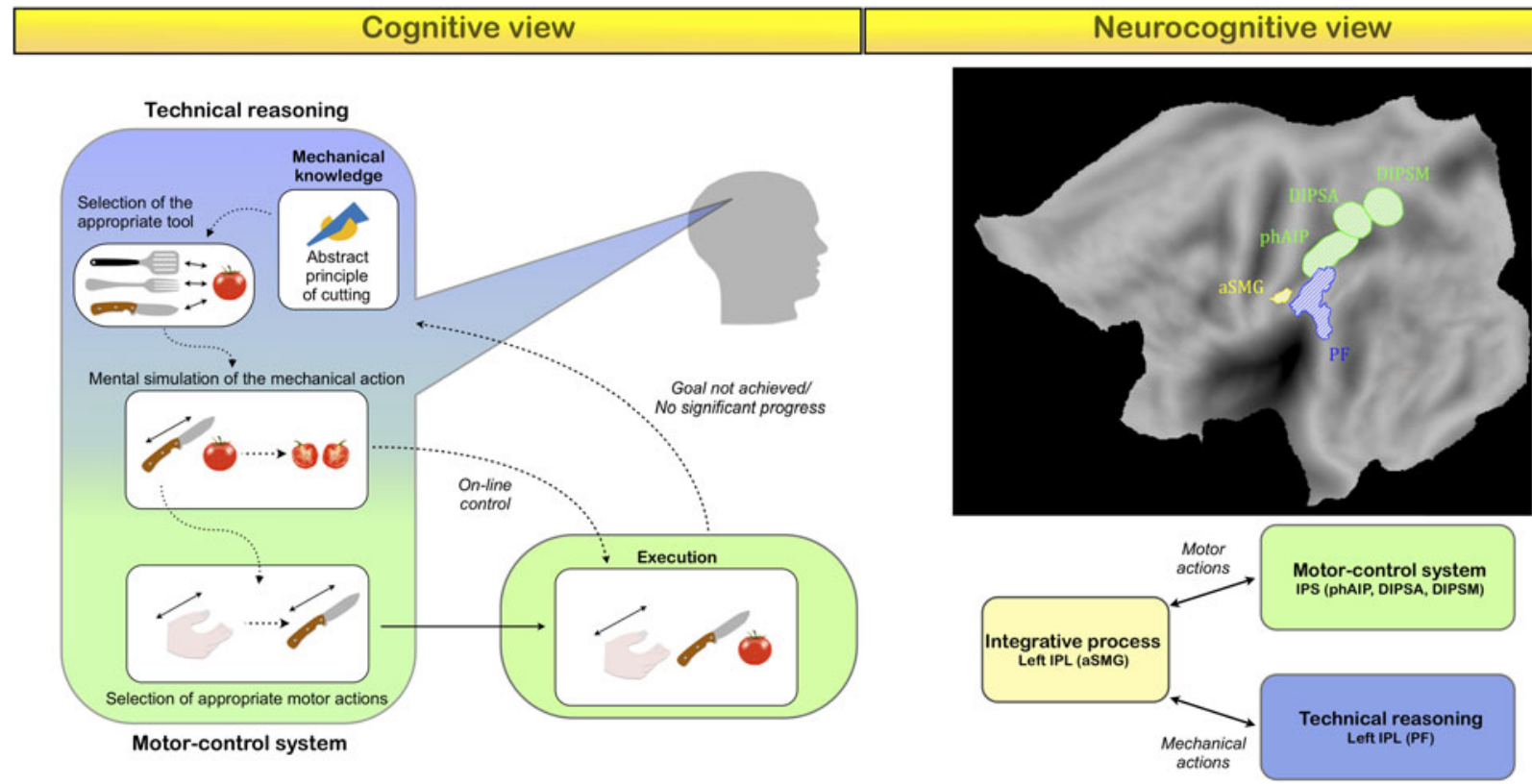

Figure 1. The technical-reasoning hypothesis. The left panel (cognitive view) illustrates the dynamics of technical reasoning (in blue) and its interaction with the motor-control system (in green). This illustration is based on an instance of use of a familiar tool (i.e., a knife). However, technical reasoning is not specific to familiar tool use because it also concerns any situations in which it is necessary to solve a physical problem, such as when making tools or during construction behavior, or in any instances requiring the understanding of the mechanics of the physical world (e.g., use of novel tools and innovation). More details about this dynamic are given in the main text. The right panel (neurocognitive view) corresponds to the neurocognitive model of the technical-reasoning hypothesis. As shown, technical reasoning might mainly involve the cytoarchitectonic area PF within the left inferior parietal lobe (IPL). The motor-control system might instead be supported by more superior parietal structures such as the intraparietal sulcus (IPS; phAIP, putative human anterior IPS; DIPSA, anterior dorsal intraparietal sulcus; DIPSM, medial dorsal intraparietal sulcus). The anterior portion of the left supramarginal gyrus (aSMG) could play a key role by biasing signals to phAIP to favor the selection of the motor actions that best suit the realization of the mechanical action generated by technical reasoning (see Orban \& Caruana 2014).

another, the expected effect is the motion of the object, which will also constrain the motor actions chosen. In other words, the technical-reasoning hypothesis assumes that no specific tool-use motor program is needed to specify how to manipulate familiar tools (see also Goldenberg 2013). In addition, if the goal is not achieved or if the action appears to be ineffective, the process returns to technical reasoning to adapt the mental simulation of the mechanical action to the present situation (e.g., change of the point of incision or orientation of the blade) or to select another tool, for instance (Fig. 1).

(5) Technical reasoning - and the dynamics of the process described above (see also Fig. 1) - is not specific to the use of familiar tools (e.g., a knife), but also concerns any situation in which a physical problem has to be solved, such as when making tools or during construction behavior, or in any situation requiring the understanding of the mechanics of the physical world (e.g., use of novel tools and innovation; Osiurak \& Heinke 2018).

The technical-reasoning hypothesis is an alternative to the longstanding tool-use motor-program hypothesis initially developed on the basis of neuropsychological findings (Buxbaum 2001; Heilman et al. 1982; Rothi et al. 1991; van Elk et al. 2014). This hypothesis focuses on the key role of motor actions (i.e., handtool relationships) in tool use and assumes that the use of familiar tools is based on specific tool-use motor programs, which are also referred to as manipulation or procedural knowledge (e.g., "knowing that a hammer is grasped at the handle and used with a back-and-forth swinging movement"; van Elk et al. 2014, p. 237). According to this hypothesis, these motor programs are concerned only with familiar tool use, but not with novel tool use, tool making, or construction. In addition, these motor programs are thought to be stored within the left inferior parietal lobe. Three lines of evidence seem to favor the technical-reasoning hypothesis over the tool-use motor-program hypothesis.

First, there is a strong link between familiar and novel tool use (e.g., unusual use of familiar tools and mechanical problem-solving including tool making) in left brain-damaged patients, who can exhibit severe difficulties when using familiar tools (Bartolo et al. 2007; Goldenberg \& Hagmann 1998; Goldenberg et al. 2007; Hartmann et al. 2005; Heilman et al. 1997; Jarry et al. 2013; Osiurak et al. 2009; see Baumard et al. 2014; for additional evidence in neurodegenerative diseases and normal aging, see also Baumard et al. 2016; Lesourd et al. 2017; Fig. 2A). Second, voxel-based lesion-symptom mapping studies have demonstrated that the same brain structure (the area PF within the left inferior parietal lobe) is systematically damaged in left brain-damaged patients with deficits in both familiar and novel tool use (Goldenberg \& Spatt 2009; Martin et al. 2016; Salazar-Lopez et al. 2016; Fig. 2B). Taken together, these findings confirm that one and the same neurocognitive process (i.e., technical reasoning, possibly supported by the left area PF) is at work whatever the familiarity of the tool-use activity, thereby ruling out the tool-use motor-program hypothesis, which assumes that only familiar tool use is supported by the left inferior parietal lobe because of the presence of preexisting tool-use motor programs. Third, we conducted a neuroimaging metaanalysis of tool use (Reynaud et al. 2016), including studies in which participants had to focus on the appropriateness of mechanical actions (i.e., tool-object relationships). The results revealed 


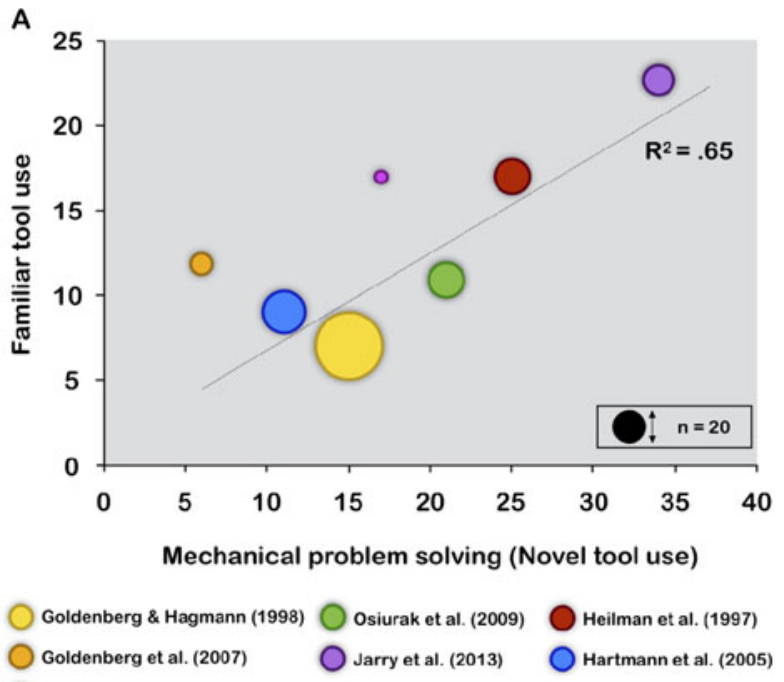

Bartolo et al. (2007)

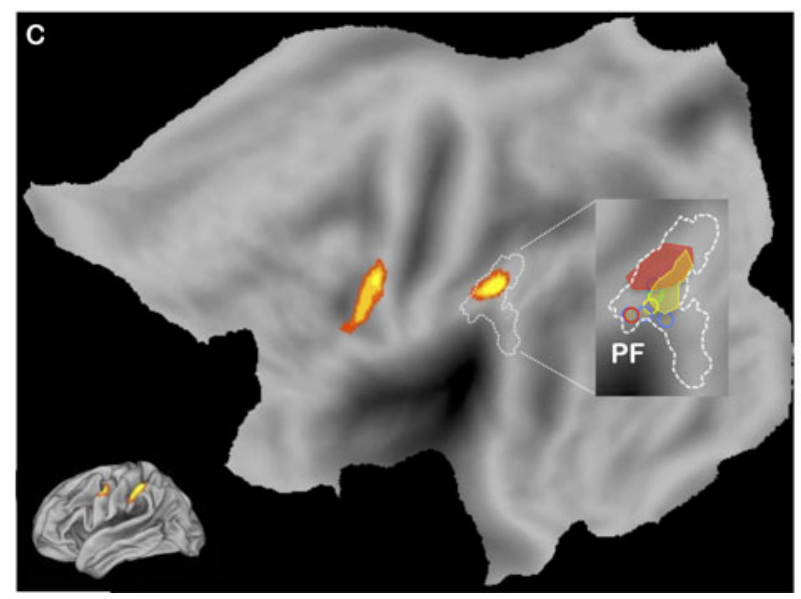

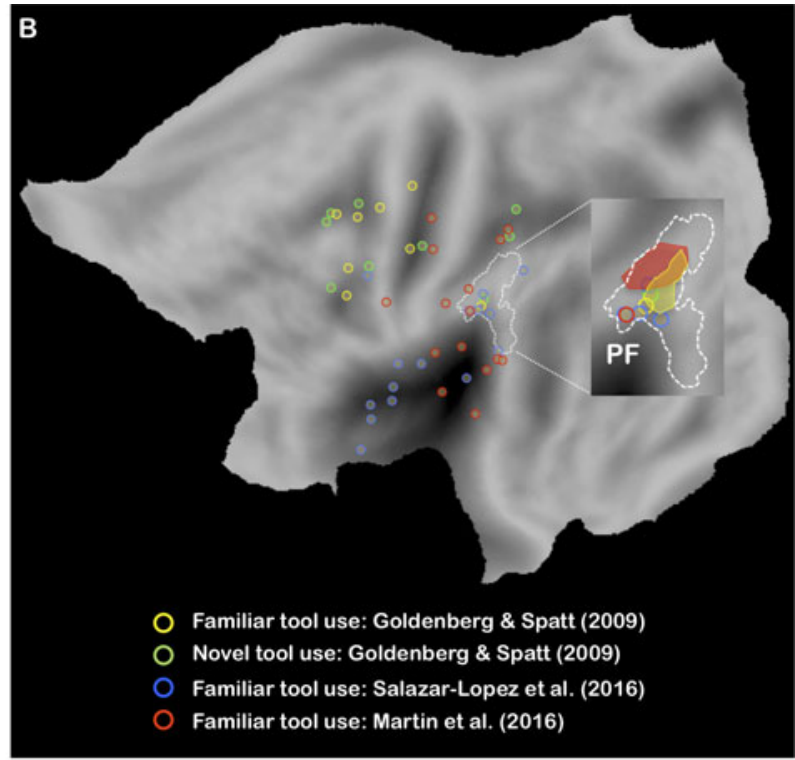

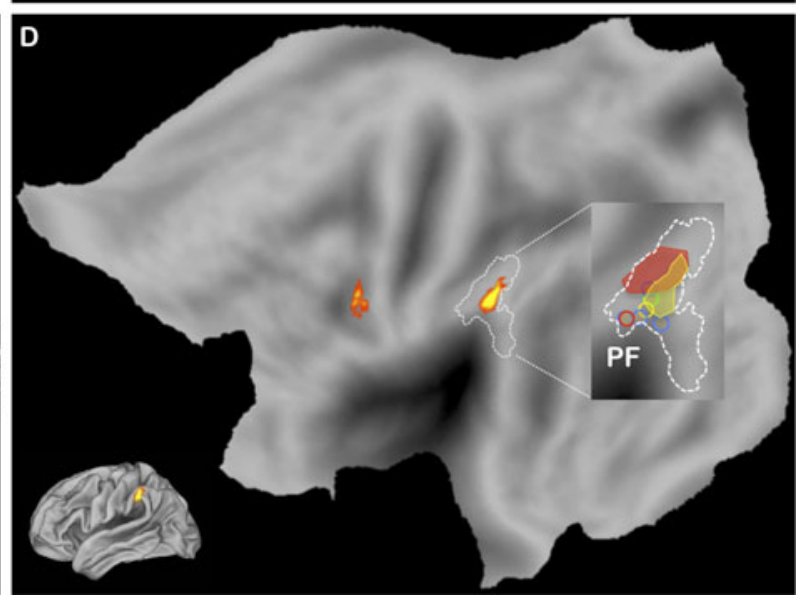

Figure 2. Evidence for the technical-reasoning hypothesis. (A) The figure depicts the strong link between familiar tool use and novel tool use in left brain-damaged patients, confirming that one and the same cognitive process (i.e., technical reasoning) is at work whatever the familiarity of the task. Each point refers to a study in which both left brain-damaged patients and healthy controls were assessed on both tasks. Patients' deficit is expressed in terms of percentage of impairment as compared to healthy controls ( $\left.\mathrm{M}_{\text {Controls }}-\mathrm{M}_{\text {Patients }}\right)$. (B) Lesion sites reported in voxel-based lesion-symptom mapping studies investigating familiar tool use and novel tool use in left brain-damaged patients. The area PF within the left inferior parietal lobe is the only brain area identified in all the studies. (C) Key finding of a recent neuroimaging meta-analysis on tool use (Reynaud et al. 2016). The analysis included studies in which healthy participants had to focus on the appropriateness of the mechanical action (tool-object relationship). Results revealed activation of the left area PF (in red in the zoomed picture), suggesting that this area is deeply involved in understanding mechanical actions (i.e., technical reasoning). (D) Key finding from a recent neuroimaging meta-analysis on tool-use observation (Reynaud et al. 2019). The results relate to the contrast between studies in which healthy participants had to observe tool-use actions minus non-tool-use actions. Again, a preferential activation of the left area PF is found (in yellow in the zoomed picture), indicating that people reason technically not only to conceive mechanical actions with tools themselves (aforementioned results) but also when watching others use tools.

activation of the left area PF (Fig. 2C), confirming that this brain area is involved in the understanding of mechanical actions (i.e., tool-object relationships) but not of motor actions (i.e., handtool relationships) as is suggested by the tool-use motor-program hypothesis.

More relevant to our purpose is the fact that we recently conducted another neuroimaging meta-analysis on action observation, in which we distinguished between studies focusing on either the observation of non-tool-use actions (e.g., grasping a ball) or the observation of tool-use actions (e.g., using a knife; Reynaud et al. 2019; Fig. 2D). The main difference between these two categories of studies is that the former include only the observation of a motor action (tool-hand relationship), whereas the latter include the observation of both mechanical (tool-object relationship) and motor actions (hand-tool relationship). Our findings indicated that the left area PF is specifically involved in the observation of tool-use actions and, as a result, of mechanical actions performed by others. In broad terms, the same neurocognitive process (i.e., technical-reasoning skills/left area $\mathrm{PF}$ ) might be at work not only when humans carry out mechanical actions, but also when they watch others doing so.

\subsection{Technical reasoning and cumulative technological culture}

The focus on the social dimension of CTC has led scientists to consider that distinct cognitive processes are at work during social (e.g., imitation) versus asocial (e.g., trial and error) learning (e.g., Tomasello 1999; Whiten et al. 2009). We acknowledge that 
specific cognitive processes can be involved in some instances of social learning because of the social dimension of the situation (see below). However, there is no reason to posit that different cognitive skills are involved in understanding the physical causalities of a situation depending on whether the situation is social or not (for a similar view, see Heyes 2012; 2016). Support for this view comes from the aforementioned neuropsychological and neuroimaging findings that have indicated that the same brain area - the left area PF - is involved not only when humans use tools, solve mechanical problems, sometimes by making novel tools, or focus on mechanical actions, but also when they observe others using tools. Further support comes from a recent series of experimental studies with micro-society paradigms in which participants had to build as high as possible a tower with metal wires (De Oliveira et al. 2019; Osiurak et al. 2020a) or a paper airplane that would fly as far as possible (Osiurak et al. 2016). In these studies, the participants performed the task as members of a chain of 10 participants, each of whom completed the task one after the other. In the observation conditions, the participants could observe other members of the group building an airplane before performing the task themselves. No communication was permitted. In the communication conditions, they could help the subsequent participant by communicating verbally with her or him. The participants' technical-reasoning and theory-of-mind skills were also assessed in additional testing sessions. The results indicated that learners' technical-reasoning skills are the best predictor of cumulative performance over generations irrespective of the kind of social learning studied (i.e., observation and communication). The technical-reasoning hypothesis of CTC represents an epistemological break with the state-of-the-art in various respects.

\subsubsection{A dormant, technical potential}

The concept of technical potential is close to that of "zone of latent solutions” (Tennie et al. 2009; 2016a; see also Bandini \& Tennie 2017). “The term 'latent solution' refers to a behavior that lies 'dormant' or 'latent' in an individual until triggered by a particular set of social or environmental cues and sufficient motivation on the part of the learner" (Tennie et al. 2016a, p. 125). In this respect, the zone of latent solutions corresponds to the room for improvement that a given species possesses in light of its cognitive skills. For instance, Tennie et al. (2009) showed that chimpanzees cannot learn a "loop technique" through social learning, simply because they cannot acquire it on their own (i.e., this technique is beyond their zone of latent solutions). Whiten et al. (2009) criticized this concept, suggesting that chimpanzees' and humans' very different CTC capacities can be understood only if the task proposed falls within the scope of a species' cognitive skills. We agree with Whiten et al. (2009) that the study of social learning and culture in any species needs to be based on tasks that are within the scope of the cognitive skills of the species. Many elegant studies have thus shown that chimpanzees, for instance, can develop cultural traditions (Horner et al. 2006; Whiten et al. 1999; 2005; 2007). However, if we acknowledge that CTC is absent or, at best, minimal in nonhumans, it is reasonable to wonder whether these difficulties might not be because of the fact that the cognitive skills they do possess are too limited to enable them to acquire technical information. In this respect, we posit that CTC emerges in humans because we possess the "dormant" technical potential (i.e., technical-reasoning skills) to acquire and improve on a vast amount of technical information. This provides the room required for technical improvement, which is a key characteristic of CTC.

\subsubsection{Imitation and innovation: Two sides of the same coin}

The distinction between imitation and innovation is commonly considered to reflect the involvement of distinct forms of learning and, as a result, of cognitive processes (social learning vs. asocial/ individual learning including trial and error, respectively; e.g., Tomasello 1999). Here, we posit that technical-reasoning skills allow humans to extract relevant technical information from a "social demonstration" (imitation), to reject irrelevant information, as well as to generate new ideas to improve the end-product (innovation). In this context, the ability to extract/reject information in an effective way is subject to interindividual differences so that people with good technical-reasoning skills are better at doing this, as evidenced by studies using micro-society paradigms (De Oliveira et al. 2019; Osiurak et al. 2016; 2020a; in press).

\subsubsection{An integrative process for social and asocial learning}

In the micro-society paradigms employed to investigate CTC in humans, the periods of social transmission and production are generally very short (e.g., 5-min period of social learning and 5 -min period of production) and there is no alternation between periods of social and individual learning (e.g., Caldwell \& Millen 2008; 2009; Morgan et al. 2015; Osiurak et al. 2016; Zwirner \& Thornton 2015). Indeed, it is very remarkable that cumulative performance can be found in such conditions, because they do not reflect what happens in everyday life, during which people generally alternate between periods of social and individual learning (i.e., exploration and practice; Whiten 2015; 2019b; see also Ingold 1998). After all, we do not assess students just 5 min after a teaching course. The risk is that we may draw conclusions that might be different if more time were given to individuals to digest what they have acquired from social learning. For instance, Morgan et al. (2015) investigated the efficacy of transmission of stone tool-making skills along the chains of participants in five different social-learning conditions (e.g., reverse engineering, observation, communication, and so on). They found that less information was lost in the communication condition than, for instance, in the reverse-engineering condition. Based on these findings, one might consider that stone tool-making skills can be transmitted only by communication, but not by observation or reverse engineering. For our part, we are not saying that communication is a less effective mode of social transmission than observation or reverse engineering (see below), merely that it remains possible that stone tool-making skills can also be transmitted via observation or reverse engineering if learners can alternate between periods of social and individual learning. Evidence for this comes from the longitudinal study by Putt et al. (2014), who found no clear difference between a communication condition and an observation condition in the making of symmetric lithic bifaces. Interestingly, these authors noticed that the individuals in the observation condition were able to learn from their mistakes and improve their flake production each week, suggesting a kind of strategy based on reasoned trial and error. Other studies have demonstrated that expertise in stone knapping requires a considerable amount of time (more than $150 \mathrm{~h}$ of practice) to progressively acquire a clear technical understanding of the task (Bril et al. 2010; see also Stout et al. 2011; 2019). In this context, technical-reasoning skills can be viewed as an integrative process that makes it possible to extract information from social learning and to test and improve it via individual 
learning ${ }^{5}$ using trial-and-error strategies that are not random but reasoned (Vaesen 2012; see also Derex et al. 2019; Whiten $2019 b) .{ }^{6}$ In addition, these reasoned trial-and-error strategies can allow humans (1) to eventually reproduce - and indirectly imitate - a technique that has not been transmitted faithfully during social interaction or (2) to converge toward the same technical invention (i.e., without direct transmission; a kind of virtual imitation) simply because the same reasoning inevitably leads to the emergence of the same technical solution (for a similar view, see the cultural attraction hypothesis; Boyer 2001; Claidière \& Sperber 2007; Claidière et al. 2014a; Morin 2015; 2016; Sperber 1996; Sperber \& Hirschfeld 2004).

\subsubsection{The basis for innovation}

Innovation can be defined as the generation of a novel learned behavior that is not the consequence of social learning or environmental induction (Ramsey et al. 2007). Innovation has been documented in a range of species including nonhuman primates (Reader \& Laland 2002). Given that CTC is absent in these species, it has been suggested that innovation alone is not sufficient for CTC (e.g., Dean et al. 2014). This view, again, stresses the key role of social, but not of non-social, cognitive skills in CTC (e.g., see Dean et al. 2012; Herrmann et al. 2007; Tomasello 1999; for a similar view, see also Hernik \& Csibra 2009). This interpretation, which minimizes the role of innovation in CTC, can nevertheless be questioned if we consider that there are major differences in terms of innovation between humans and nonhumans (see Gruber 2016; Gruber et al. 2011; for evidence indicating difficulties in tool innovation in nonhuman primates). One of these differences concerns the uniquely human ability to combine techniques, as in the case of secondary tool use (i.e., the use of one tool to create another; Shumaker et al. 2011). Interestingly, modeling work has shown that innovation in humans might primarily result from technical combinations rather than from novel inventions or modifications (Lewis \& Laland 2012). Thus, even if innovation is not unique to humans, humans might be unique in exhibiting combinatory innovation. At a cognitive level, this ability clearly reflects analogical reasoning skills (Vaesen 2012). For instance, after learning a percussion technique to crack nuts (i.e., acquisition of the mechanical knowledge "percussion"), an individual can transfer it to the context of stone fracture to increase the production of flakes. One of the characteristics of technical reasoning is that it is analogical. Therefore, technical reasoning is an appropriate candidate to account for technical innovation in humans ${ }^{7}$ (for a similar view, see Haidle 2014; Penn et al. 2008b; Vaesen 2012; Wolpert 2003). Importantly, the analogical component of technical reasoning does not allow humans to systematically generate appropriate new technical solutions. Sometimes - or even often - the transfer of a technique to a new context can lead to failures, which can nevertheless be viewed as inventions at the individual level (for a distinction between technical invention/individual level and innovation/group level, see Wynn \& Coolidge 2014). Nevertheless, we assume that this reasoned trial-and-error strategy might be an important engine for innovation, whatever the outcome (innovation or "ineffective inventions"/failures).

\subsubsection{Technical reasoning is involved in all forms of social learning}

As shown in Table 1, we have identified three main forms of social learning: reverse engineering (indirect), observation (direct), and communication (direct). Any theory that assumes that CTC originates in uniquely human social cognitive skills (e.g., Tomasello et al. 1993) must also account for the fact that signs of CTC are also observed in reverse-engineering conditions in which models and learners cannot interact directly. Despite this, studies using micro-society paradigms have reported cumulative performance in such conditions (Caldwell \& Millen 2009;

Table 1. Behavioral classification of forms of social learning

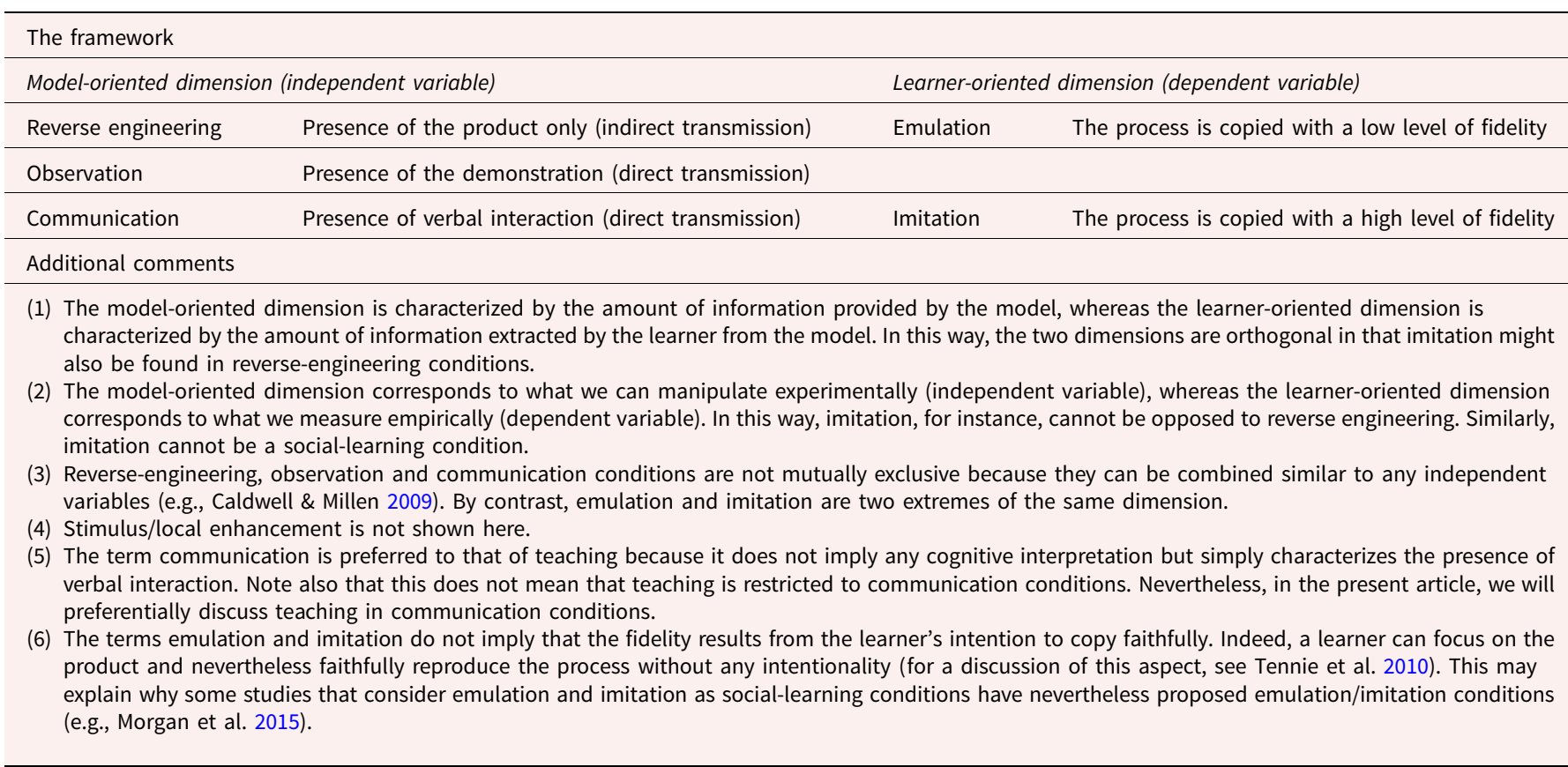


Derex et al. 2019; Zwirner \& Thornton 2015; see also Caldwell et al. 2012; 2018), although the degree of accumulation is generally lower than in observation or communication conditions (e.g., Caldwell \& Millen 2009). However, it should be remembered that it is already an achievement to observe cumulative performance in such experimental conditions in which there is very limited time and no alternation between periods of social and individual learning. Therefore, if signs of CTC in reverse-engineering conditions are observed in humans, then this suggests that human non-social cognitive skills are sufficient for the emergence of CTC (Vale et al. 2012). Interestingly, in the neuropsychological literature, the idea that people can infer how to use an artifact from its physical structure has already been linked to concepts similar to technical reasoning (e.g., Goldenberg 2013; Goldenberg \& Hagmann 1998; Hodges et al. 1999; 2000; Humphreys 2001). Thus, in line with this proposal, we assume that technical-reasoning skills can be useful in reverse-engineering conditions because they make it possible to infer the making process (see also Vaesen 2012; Zwirner \& Thornton 2015). In this respect, the technicalreasoning hypothesis offers a parsimonious framework for the cognitive study of CTC, suggesting that this reasoning is necessary for the emergence of CTC irrespective of the social-learning conditions (see also Vaesen 2012). It should be noted, however, that this assumption is consistent with the idea that social cognitive skills could also be involved in direct social-learning conditions, thus making it possible to boost CTC (see below).

\subsection{Mechanical knowledge is "a kind of magic"}

One false belief about technical-reasoning skills is that these skills should lead to a full understanding of any physical situation, permitting the extraction of everything that is relevant from a social interaction but no more than that (Boyd et al. 2011; 2013; Derex et al. 2019). The following quote provides a good illustration of this: "You see that your uncle's bow shoots farther than yours and notice that it is thicker, but less tapered, and uses a different plait for attaching the sinew. You copy all three traits, even though in reality it was just the plaiting that made the difference" (Boyd et al. 2011, p. 10922). According to Boyd et al. (2011; see also Derex et al. 2019), the fact that humans can also copy irrelevant traits (as illustrated by this quote) is at odds with the idea that technical-reasoning skills are fundamental to CTC - because otherwise humans would copy only the relevant trait (e.g., the plaiting). This omniscient view of technical reasoning is erroneous. It is not because we possess technical-reasoning skills that we are able to spontaneously understand all the dimensions of a physical phenomenon. The reason is that technical reasoning is based on mechanical knowledge, which, similar to any form of knowledge, is a belief but not necessarily true.

This aspect has been elegantly demonstrated by Baillargeon and colleagues, who have shown how the principle of support evolves in early childhood (6 months: Infants consider any amount of contact between the object and the support sufficient for the object to be supported; 6-9 months: Infants expect an object to remain stable if a significant portion of its surface is in contact with the support; 9 months: Infants understand that other features such as the mass distribution of an object is critical to determine whether a support is appropriate or not, and so on; Baillargeon \& Hanko-Summers 1990; Baillargeon et al. 1992; Needham \& Baillargeon 1993). These findings indicate that mechanical knowledge can be acquired relatively early in childhood and can continue to grow over time (Remigereau et al. 2016), even during adulthood. In this respect, mechanical knowledge is never completely accurate and can be the basis for some "magical" beliefs such as thinking that any amount of contact between an object and a support is sufficient for the object to be supported. Nevertheless, even such magical beliefs can be useful for inferring potential outcomes in the environment (i.e., causal and analogical reasoning, the two dimensions of technical reasoning). However, over time, these inferences can be invalidated through a kind of Bayesian learning, leading the individual to progressively acquire more accurate knowledge about physical phenomena (i.e., technical expertise).

Recently, Derex et al. (2019) reported a micro-society paradigm in which participants had to improve a physical system (i.e., a wheel that traveled down a 1-m-long inclined track). They found cumulative performance in that the wheel traveled farther in late than in early generations. However, there was no increase over generations in terms of "causal understanding," which was assessed with an additional decision task in which the participants had to choose the best wheel configurations. For the authors, these findings demonstrate that $\mathrm{CTC}^{8}$ can emerge without causal understanding (i.e., technical reasoning). This conclusion is subject to considerable reservations. The authors' main argument was that CTC occurred even though the participants did not develop a full understanding of the physical system, with most of them producing simplistic models related to a salient dimension (i.e., inertia and center of mass) rather than multidimensional causal theories. This is clearly at odds with an omniscient view of technical reasoning. However, it is consistent with the aforementioned idea that mechanical knowledge is the basis for some magical beliefs and, as a result, is not completely accurate. Thus, based on this inaccurate knowledge, the participants were able to produce effective - but not the most effective - solutions and this explains the presence of CTC over generations. It is also consistent with the fact that Derex et al. (2019) themselves reported signs of causal understanding, stressing that their "participants did not randomly explore the parameter space" (p. 448; i.e., reasoned trial and error, see above; see also Kendal 2019). In broad terms, these findings do not provide clear evidence against the link between CTC and technical-reasoning skills.

\subsection{Emulation versus imitation}

As shown in Table 1, we suggest a revised classification of the different forms of social learning that diverges from previous proposals (Heyes 1994; Hoppitt \& Laland 2013; Whiten \& Ham 1992; Whiten et al. 2003; 2009). This new classification is organized around two dimensions, namely the information provided by the model to the learner (i.e., model-oriented dimension) and the amount of information extracted by the learner from the model (i.e., learner-oriented dimension). In the experimental context, these two dimensions can be viewed as the independent variable and the dependent variable, respectively: We experimentally manipulate the amount of information provided by the model to investigate the amount of information extracted by the learner. In this classification, emulation (copying of "results") and imitation (copying of "actions" and "results") are distinguished on the basis of the correspondence between the model's behavior and the learner's behavior. In addition, there is no a priori knowledge concerning the model-oriented 
dimension in that a learner may "imitate" a model even in a reverse-engineering condition if she or he reproduces absolutely all the same actions that are performed by the model. In light of this reservation, a condition should not be considered $a$ priori as imitative or emulative because this presupposes that participants are already ready to copy the different actions (e.g., Morgan et al. 2015; Schillinger et al. 2015; Zwirner \& Thornton 2015). Terms such as "reverse engineering/end-product" or "observation/action" conditions allow us to overcome this classification issue (e.g., Caldwell \& Millen 2009; Wasielewski 2014).

The direct link between imitation and CTC has been repeatedly stressed in the field (Boyd \& Richerson 1996; Derex et al. 2013b; Galef 1992; Henrich \& Gil-White 2001; Tennie et al. 2009; 2012; Tomasello 1999; Tomasello et al. 1993; 2005). The critical question, however, is what is imitated? To tackle this question, we propose to distinguish between what we have called mechanical actions (i.e., tool-object relationships) and motor actions (i.e., hand-tool or, more generally, body-tool relationships; also called bodily actions).

The first possibility is to focus on motor actions. This would lead us to consider that humans alone copy the motor actions of the model (e.g., Henrich \& Gil-White 2001; Tennie et al. 2009; Tomasello et al. 1987; see also Köhler 1959; Lotem et al. 2017). This possibility has two main limitations. First, it implies experimental paradigms in which only one mechanical action is proposed and can be solved using different motor actions, preferentially performed by different body parts (e.g., head vs. hand; see Gergely et al. 2002). However, even with such a simple methodology, scoring issues can arise. For instance, what if a model uses the right hand and the learner the left hand (see Wohlschläger et al. 2003)? Is this an instance of imitation or emulation? This methodological issue necessarily grows in importance as the number of mechanical actions increases, thereby limiting the study of the link between imitation and CTC. Second, in line with the ideomotor principle (Brass \& Heyes 2005; Wohlschläger et al. 2003; see also Osiurak \& Badets 2014; 2016), the learner can attempt to reproduce the same mechanical action as the model, and then indeed perform the same motor action, simply because this motor action is the most economical for both of them at a biomechanical level (see Claidière et al. $2014 \mathrm{~b}$ for a convergent cultural evolution in baboons based on biomechanical constraints). This is a kind of "sunflower effect": All the sunflowers follow the sun throughout the course of the day, leading them to perform the same rotation; and yet, sunflowers do not imitate each other. In broad terms, this first possibility, which focuses on motor actions, is hard to demonstrate experimentally and is also partly ruled out by evidence indicating that children reproduce behaviors better when information about the mechanical rather than the motor action is provided (Flynn \& Whiten 2013). In other words, mechanical actions and non-motor actions might be the vital basis for any social transmission.

This leads us to a second possibility: the focus has to be on mechanical actions (Whiten et al. 2009). Therefore, a learner can be considered to be imitating a model if she or he reproduces the same mechanical action as the model. A good way to explore this possibility is to use a "two-action" paradigm consisting of offering the choice between two mechanical actions that can be used to achieve the same goal (e.g., Horner \& Whiten 2005; Horner et al. 2006; Whiten et al. 2005). A significant body of evidence has been gathered using this paradigm and clearly demonstrates that nonhumans can not only exhibit imitation by reproducing the same mechanical action as that performed by a model, but also establish this as a tradition within their group (Bonnie et al. 2007; Whiten et al. 2005; for review, see Whiten \& van de Waal 2017). In other words, these results invalidate the idea that (1) only humans can imitate and (2) CTC is necessarily based on imitation. More interestingly, it has also been shown that both humans and nonhumans can exhibit flexibility by alternating between imitation and emulation (e.g., Horner \& Whiten 2005). More generally, these findings question the idea that humans are imitators and nonhumans are - at best emulators (Whiten et al. 2009; see also Caldwell et al. 2012; Dunstone \& Caldwell 2018).

A third possibility needs to be considered. In nonhumans, imitation generally concerns one mechanical action (e.g., using either a slide or a lift method to open a door, Horner et al. 2006; making a composite tool from two components, Price et al. 2009) or a sequence of independent mechanical actions (hereafter called sequential mechanical actions; e.g., twisting a bolt, spinning a pin, and turning a handle; Whiten 1998), but not a combination of interdependent mechanical actions (hereafter called combined mechanical actions) that make it possible to achieve a technical goal (e.g., making a simple spear by producing a flake tool to cut down a tree and carve the spear, Haidle 2010). By contrast, in humans, micro-society paradigms have shown that cumulative performance can emerge over generations even when participants have to build complex artifacts by means of combined mechanical actions (e.g., Caldwell \& Millen 2009; Osiurak et al. 2016; Zwirner \& Thornton 2015). Thus, the main difference between humans and nonhumans may lie in the ability to learn such combined mechanical actions from a model (for a similar view, see Whiten et al. 2003).

Some cognitive archeologists have suggested that the complexification of human technology over time might be explained by a specific enhancement of working memory (Wynn \& Coolidge 2007; see also Haidle 2010; 2014). In line with this idea, it can be hypothesized that humans might be able to learn combined mechanical actions from models because of their enhanced working-memory skills. This hypothesis does not explain the qualitative difference between sequential and combined mechanical actions. Being able to maintain multiple mechanical actions in memory for a period of time is cognitively different from being able to combine them into a purposeful chain of interdependent mechanical actions. Furthermore, working memory is not a cognitive mechanism that is used to generate content, but instead temporarily stores content that is processed by other cognitive mechanisms (see Osiurak 2017). Therefore, another hypothesis is that nonhumans might have difficulties understanding the physical principles underlying mechanical actions because of their lack of technical-reasoning skills. This would mean that they can, at best, imitate sequential mechanical actions but not combined mechanical actions. By contrast, the human ability to understand mechanical actions could enable us to store these more easily in working memory and thus permit us to reproduce the combined mechanical actions viewed in a model. The corollary is that the degree of technical expertise (i.e., the amount of mechanical knowledge possessed by an individual) modulates the level of copying, as shown in other domains such as chess where masters can quickly recall a very high number of "logical" chess configurations (e.g., Chase \& Simon 1973). If we adopt this viewpoint then the degree of technical expertise is not a bias for CTC that needs to be controlled (e.g., Derex et al. 2019; Reindle \& Tennie 2018; Tennie et al. 2012), but rather a key aspect to be studied. 
In addition, the fact that technical-reasoning skills can modulate the amount of information extracted in the case of combined mechanical actions can also explain why imitation and emulation are also flexible in humans, sometimes leading to innovation (for a similar view concerning technical expertise based on long-term working memory models, see Wynn \& Coolidge 2014). To illustrate this, imagine Einstein explaining to you his theory of relativity. If you possess absolutely no knowledge about physics and mathematics, you may be able to reproduce at best $1 \%$ of his theory, even though you make a considerable effort to maintain a lot of information in working memory. If you are a physics graduate student, you may reproduce $20 \%$. However, if you are the new Einstein, you may reproduce $80 \%$, the remaining $20 \%$ corresponding to the improvement you make to the theory (i.e., innovation). However, the fact remains that if someone asks you to explain the entirety of the theory, you will be able to do so.

In sum, this third possibility, which focuses on combined mechanical actions, offers a revised view of the distinction between emulation and imitation, thereby departing from the idea that copying in humans is both process- and product-oriented because we alone are able to infer the mental states of others and represent others' actions as intentional (e.g., Tennie et al. 2009; 2012; Tomasello et al. 2005; for a similar view, see Csibra \& Gergely 2006; 2009). Indeed, it is one thing to have the intention to imitate but another to be able to do so. After all, even if you intend to reproduce what Einstein is explaining, you may still copy no more that $1 \%$ if you are a novice in physics. Therefore, beyond the question of whether humans alone can imitate or whether imitation is the basis for CTC, a more critical question is whether humans are able to copy a great number of mechanical actions without possessing the technical ability to implement them. Our answer is that they probably do not.

\subsection{Interim conclusion}

Non-social cognitive skills are commonly excluded from the discussion about the origins of CTC. The technical-reasoning hypothesis offers a viable alternative to this view by assuming that CTC could not emerge without a specific technical potential based on non-social cognitive skills that allow us to reason about our physical world. These skills are involved in (1) both asocial/ individual and social learning, (2) imitation and innovation, (3) combinations of mechanical actions, and (4) can develop over time, leading to interindividual differences in terms of technical expertise. In this perspective, social learning is not the cause, but a catalyst of CTC, enabling humans to acquire technical information more quickly than through asocial learning. In the next two sections, we will discuss the potential role of two - apparently distinct cognitive skills (i.e., theory-of-mind and metacognitive skills) that can also contribute to CTC by facilitating social learning but without being necessary for its emergence.

\section{Theory of mind}

\subsection{Theoretical framework}

Theory of mind refers to the ability to attribute mental states to oneself and others (Bruner 1983; Harris 1991; Mead 1934; Piaget 1932; Premack 1988; Tomasello et al. 1993; Whiten 1991). ${ }^{9}$ The fact that this ability also concerns one's own mental states has led some authors to question the cognitive independence between theory-of-mind and metacognitive skills (e.g.,
Carruthers 2009; Dunstone \& Caldwell 2018; Flavell 2000; Shea et al. 2014; Tomasello et al. 1993). We will address this relationship in more detail below. It has been suggested that CTC may originate in uniquely human theory-of-mind skills (i.e., the theory-of-mind hypothesis of CTC; Boyd \& Richerson 1996; Herrmann et al. 2007; Tomasello 1999; Tomasello \& Call 1997; Tomasello et al. 1993; 2005). ${ }^{10}$ There are four main hypothesized manifestations of this involvement. The first is imitation. According to the theory-of-mind hypothesis, humans imitate others because their theory-of-mind skills might help them to "conceive" that others behave intentionally and, as a result, that they can be a source of information (see also Csibra \& Gergely 2009). Support for this idea comes initially from studies indicating that humans imitate whereas nonhumans emulate (e.g., Nagell et al. 1993; Tomasello et al. 1987). However, as stressed above, the humans/imitation versus nonhumans/emulation distinction is controversial and we will therefore not discuss it further (but see below for the question of overimitation in children). The other three manifestations are: cooperation, teaching, and opacity. In the next sections, we address these manifestations in turn.

\subsection{Cooperation}

Resource-sharing-based division of labor characterizes all human cultures (Gibson 1993; Ingold 1993; Reynolds 1993; Whiten \& Erdal 2012). This may concern, for instance, foraging (huntinggathering) or childcare (Whiten \& Erdal 2012). This cooperative dimension may be unique to humans, with nonhumans generally being more engaged in competition during their social interactions (Moll \& Tomasello 2007; Tennie et al. 2016b). The theory-of-mind hypothesis of CTC offers a theoretical framework for a narrower definition of cooperation as "shared or joint cooperative activities" during which participants (1) share a joint goal, (2) take on reciprocal and complementary roles to reach this joint goal, and (3) are motivated to help one another accomplish their roles if needed (Moll \& Tomasello 2007). A corollary is that working together allows individuals to pool their knowledge, thereby offering (4) the opportunity for separate solutions to be combined (Tomasello 1999; Tomasello \& Call 1997; see also Dean et al. 2014). Indeed, theory-of-mind skills might be a key factor favoring the development of cooperation in humans. This can explain how two individuals can share a joint goal and be motivated to help one another (points 1 and 3 above, respectively). However, the question is whether theory-of-mind skills are sufficient to support the emergence of complementary roles (point 2 above) as well as the combination of solutions (point 4 above).

Let us begin with the question of complementary roles. Cooperation can be defined in different ways depending on the role played by each participant (for a classification, see Boesch $\&$ Boesch 1989). In this respect, a joint cooperative activity is an instance of collaboration (Boesch \& Boesch 1989), also called heterotechnic cooperation (Reynolds 1993), in that the different participants do not perform similar actions in time and space (i.e., coordination) but different and complementary actions. More rudimentary forms of cooperation have been documented in nonhumans (see Boesch 2002). However, although heterotechnic cooperation may be characteristic of all human cultures, evidence in favor of such forms of cooperation is rare in nonhumans (Boesch 2002; Boesch \& Boesch 1989; Boesch \& Boesch-Achermann 2000) and subject to debate (see Moll \& Tomasello 2007). Heterotechnic cooperation can clearly contribute to CTC by allowing humans to engage in and develop effective 
technical activities they could not perform alone. Imagine, for instance, a group of seven individuals intending to lift a heavy piece of wood to strengthen the top of a cabin. To do so, they use a pulley technique. The piece of wood is tied with a rope and the rope passes over a strong branch situated above the cabin. Five individuals are needed to pull the rope to lift the piece of wood. Two additional individuals are also needed (1) to direct the piece of wood toward the top of the cabin using a pole and (2) to take hold of it and attach it to the roof. The theory-of-mind hypothesis can explain the motivation that these individuals have to act cooperatively, but not how the idea of dividing this activity into different mechanical actions has arisen.

Our assumption is that this type of cooperative activity is based on a combination of mechanical actions (e.g., tying and pulley) intended to solve a physical problem. It is necessarily the ability to conceive these different mechanical actions (through technical reasoning) that can transform any cooperative activity into a heterotechnic cooperative activity. Additionally, the cooperation between humans is anecdotal here. A workhorse or a tractor could have pulled the rope so that even a single individual could have solved this problem alone and our example of heterotechnic cooperation could simply be motivated by necessity because of the available technical resources (i.e., no workhorse or no tractor). This also leads us to address another aspect, which is that humans can also be viewed by their conspecifics as technical resources (e.g., workforce) in the same way as nonhumans. The history of mankind has clearly demonstrated that humans can, unfortunately, exploit nonhuman animals and other humans (e.g., slavery and colonization) and sometimes use them as technical resources to build exceptional constructions (e.g., Egyptian pyramids).

The same rationale can be applied to combinations of solutions. Although engaging in cooperative activities can lead to the sharing of technical solutions and, potentially, new combinations of solutions, theory-of-mind skills do not adequately account for the integration of these solutions within a coherent representation of mechanical actions (see above for a discussion of combination in the context of imitation and innovation). In sum, although theory-of-mind skills could favor cooperation, technical-reasoning skills remain necessary to transform this cooperation into heterotechnic cooperation or to use it to produce new combinations of solutions.

\subsection{Teaching}

Teaching can be broadly defined as behavior that facilitates learning in others (Kline 2015; Thornton \& Raihani 2008). In recent years, evidence has accumulated for teaching behavior in nonhumans (e.g., Franks \& Richardson 2006; Thornton \& McAuliffe 2006). Although these instances correspond to more rudimentary forms of teaching (e.g., scaffolding; Csibra 2007), they do not satisfy the strict definition of "direct active teaching," during which an individual engages in teaching as a consequence of her or his own belief (which may or may not be substantiated) about the likely effect on the learner (Caldwell et al. 2018; Caro \& Hauser 1992; Csibra \& Gergely 2006; Kline 2015). To date, no clear examples of active direct teaching have been identified in nonhumans (Csibra 2007; Galef 1998; Kline 2015; Tennie et al. 2009; but see Boesch 1991). For this reason, the term teaching will be used in the following to refer to direct active teaching. Similar to imitation, it has been suggested that teaching is a precursor of CTC (Tennie et al. 2016a; Tomasello 1999; Tomasello et al. 1993; see also Dean et al. 2012; 2014). Indeed, it can be hypothesized that theory-of-mind skills might be critical for forming a representation of what others understand about a given task. Based on this, an individual can become a teacher by orienting the attention of another individual (i.e., the learner) toward relevant information and providing appropriate feedback during the task (Csibra \& Gergely 2006; Dunstone \& Caldwell 2018). Strictly speaking, teaching can occur in both communication and observation conditions (Table 1).

However, studies that have included observation conditions have not generally reported data indicating that the model is clearly engaged in teaching (e.g., by slowing down the demonstration). By contrast, the use of verbal information in communication conditions makes it easier to see that the model is engaged in teaching based on her or his hypothesized theory-of-mind skills. The idea of a direct link between CTC and teaching is supported by a significant body of evidence acquired using microsociety paradigms, which has shown that cumulative performance is better (or that less information is lost over generations; Morgan et al. 2015) in communication conditions than in observation or reverse-engineering conditions (Caldwell \& Millen 2009; De Oliveira et al. 2019; Osiurak et al. 2016; Zwirner \& Thornton 2015; see also Caldwell et al. 2018). Despite these findings, the teaching/theory-of-mind hypothesis remains unsatisfactory as an explanation of CTC for several reasons.

First, even if communication conditions favor CTC in micro-society paradigms, CTC can also be found in observation conditions and, more problematically, in reverse-engineering conditions, in which models and learners do not interact at all. Although the complexity of the technical information that could be transmitted in such conditions may be limited, the fact remains that less complex forms of CTC could emerge without theory-of-mind skills. Consequently, given that technicalreasoning skills may be involved in all forms of social learning within a technical context, CTC could instead originate from these skills whereas nevertheless still being boosted by the different contributions of theory-of-mind skills (i.e., cooperation and teaching; for a similar view, see Caldwell et al. 2018). Second, the impact of teaching on CTC might also be overestimated in that the ethnological record of hunter-gatherer cultures reports that teaching plays a minimal role compared to observational learning (Hewlett et al. 2011; MacDonald 2007). Third, the idea that teaching is critical to CTC also implies that teachers are reliable sources of information for learners. However, this depends on several factors, such as the teacher's theory-of-mind skills, which are necessarily subject to interindividual differences in the same way as any other cognitive skills. In other words, even if a teacher is motivated to orient the learner's attention toward relevant information, this orientation can be untimely and slow down the learning process by preventing the learner from focusing on other aspects of the task that are actually more relevant to her or him (Putt et al. 2014; for a similar conclusion in a written communication condition, see Derex et al. 2019). Fourth, recent evidence from micro-society paradigms indicates that learners' technical-reasoning skills are a better predictor of cumulative performance than teachers' theory-of-mind skills not only in observation but also in communication conditions (De Oliveira et al. 2019; Osiurak et al. 2016; 2020a). Even if teaching can facilitate the transmission of technical information, what a learner can acquire remains first and foremost determined by her or his technical-reasoning skills. 
Although teaching certainly remains a very effective way of transmitting information, it might nevertheless not be the necessary condition for CTC. Interestingly, teaching could play a key role in certain specific situations in which the learner lacks information. This can be the case when someone attempts to reproduce a tool whose making process is opaque (see below). More generally, teachers' theory-of-mind skills can be particularly relevant - and even necessary - when the learner and the teacher cannot directly share information about a specific technical task (i.e., nothing-is-present situations; see Osiurak et al. 2020a). For instance, imagine a teacher explaining to a learner how to build a shelter in the absence of any raw material. In this situation, the teacher has to guide the learner through the building process using only her or his words. Theory-of-mind skills might play a critical role here in allowing the teacher to form an accurate representation of what the learner already knows as well as in permitting the use of terms that are comprehensible to the learner.

We recently tested this hypothesis in a micro-society paradigm that included a communication condition in which learners and teachers could communicate without sharing visual information (i.e., they were sitting on two chairs positioned back to back; Osiurak et al. 2020a). We confirmed the hypothesis by observing that teachers' theory-of-mind skills were the best predictor of cumulative performance, along with learners' technical-reasoning skills. These findings indicate that teaching could help boost CTC by freeing humans from the need to be in concrete situations (i.e., presence of materials and demonstration) to acquire and transmit new technical information. It should be noted that this supportive role is far from unimportant given the adaptive value of being able to transmit information in the absence of concrete situations. However, this boosting role might be systematically conditioned by learners' technical-reasoning skills.

\subsection{Opacity}

The terms opacity/transparency refer to the amount of information that an individual can extract from scrutinizing an artifact. An artifact is transparent if it provides adequate information about how it is built (e.g., a simple spear), and opaque if not (e.g., a spear with a split-based bone point). ${ }^{11}$ Given the lack of information directly provided by opaque artifacts, it has been repeatedly argued that more complex forms of social learning (e.g., imitation and teaching) might be required to transmit this (Boyd \& Richerson 1985; Caldwell \& Millen 2009; Caldwell et al. 2018; Csibra \& Gergely 2006; 2011; Derex et al. 2013b; Schillinger et al. 2015; Tennie et al. 2012; Wasielewski 2014). Support for this view comes from studies indicating that the building of transparent artifacts can be transmitted through many forms of social learning, including reverse engineering, whereas the building of opaque artifacts can be transmitted in communication and observation conditions, but to a lesser extent in reverse-engineering conditions (e.g., Caldwell et al. 2018; Derex et al. 2013b; Morgan et al. 2015; Wasielewski 2014). The theory-of-mind hypothesis of CTC provides an appropriate framework for interpreting these findings in that teachers' theory-of-mind skills might play a critical role when the learner lacks information, for example in nothing-is-present situations or when an artifact is opaque. Nevertheless, this hypothesis might place exaggerated emphasis on what the teacher can provide (based on theory-of-mind skills) compared to what the learner can understand (based on technical-reasoning skills). More specifically, the successful transmission of an opaque artifact may depend first and foremost on the learner's technical expertise, meaning that a technical expert might be able to reproduce an opaque artifact in all social-learning conditions (i.e., reverse engineering, observation, and communication), whereas an individual with little technical expertise might encounter difficulties whatever the condition. Let us discuss this aspect in more detail.

The notions of opacity/transparency commonly refer to an essential property of an artifact. An artifact is either opaque or transparent, and this is true for any individual. In line with James (1890/2007), we propose an epistemological shift by considering that "there is no property that is absolutely essential to any one thing" (see also Osiurak et al. 2010). The corollary is that the opacity of an artifact is phenomenologically based on the learner's technical expertise. For instance, it has been suggested that CTC was observed in a reverse-engineering condition in which the task required the participants to make a paper airplane, that is, a transparent artifact (e.g., Derex et al. 2013b). Our experience with this task has taught us that many participants can find it difficult to reproduce the folding sequence by observation or to extract it by scrutinizing airplanes built by their predecessors. In other words, the assumption that the paper-airplane building task is fundamentally a transparent task for any individual is questionable. Given the link we found between cumulative performance and learners' technical-reasoning skills in such a task (Osiurak et al. 2016), this leads us to consider that the level of technical expertise can make the artifact transparent for some participants and opaque for others. It would therefore be interesting to propose a similar task to origami experts. Because of their expertise in folding techniques, all such artifacts should be transparent and they should achieve cumulative performance whatever the social-learning condition.

In broad terms, the major issue an individual faces when attempting to reproduce an artifact is to extract information from it. We posit that this extraction can be mediated by two factors. The first is the level of technical expertise, which can make an artifact opaque for some individuals, but transparent for others. The second is the information provided by the model. In cases where learners possess little technical expertise that is relevant to the task, teachers' theory-of-mind skills may help to compensate for this lack. This learner-centered perspective diverges from the position according to which the level of expertise is viewed as a confound in the study of CTC (Derex et al. 2019; Reindle \& Tennie 2018; Tennie et al. 2012). Taken to its logical conclusion, this position implies that CTC should be studied only with inexperienced participants who have no previous knowledge about the task. This position is difficult to sustain because it would exclude the ethnological record on hunter-gatherer cultures in which individuals are never inexperienced. In addition, it suggests that the best way to explore CTC would be to study only children (Reindle \& Tennie 2018), presupposing that they have no prior knowledge about the task - which is always difficult to demonstrate.

The motivation for this methodological choice is also debatable because it is more than likely that the innovative component of CTC does not develop in children but in adults, who are necessarily not inexperienced. In line with this idea, Reindle and Tennie (2018) failed to obtain cumulative performance in a micro-society paradigm with children. Nevertheless, they observed the presence of cultural lineages in the different transmission chains, a finding very similar to those obtained in nonhumans. This study is instructive in showing that it is precisely by 
controlling for the so-called confound (i.e., technical reasoning) that CTC, and its innovative component in particular, can be suppressed. The technical-reasoning hypothesis offers a clear interpretation for these findings. Children do not possess a level of technical expertise that is sufficient to allow them to detect irrelevant information in a model and this makes it difficult for them to generate more effective solutions. Even if their level of technical-reasoning skills can allow them to reproduce a solution (even in reverse-engineering conditions; Reindle et al. 2017), their innovative skills remain limited (Beck et al. 2011; Cutting et al. 2014). In sum, technical-reasoning skills are not a confound, but perhaps the key cognitive skills explaining CTC and its innovative component. This is an aspect that has commonly been overlooked in the literature (for a similar viewpoint, see Caldwell et al. 2018).

\subsection{Interim conclusion}

The emphasis on the social dimension of CTC has led researchers to assume that social cognitive skills are the precursor of CTC. Teachers' theory-of-mind skills can favor cooperation or play a boosting role in situations where learners lack information (e.g., nothing-is-present situations or situations in which the process used to make an artifact is opaque for the learner). Nevertheless, the emergence of heterotechnic cooperation or the amount of information that can be extracted from social transmission is fundamentally dependent on (learners') technicalreasoning skills. The learner-centered perspective defended here has the merit of refining previous proposals based on a teachercentered perspective. For instance, Csibra and Gergely (2006) stressed that teaching (or pedagogy) is characterized by the explicit manifestation of generalizable knowledge by a teacher, a key aspect that could distinguish active direct teaching in humans from other forms of teaching in nonhumans (see Csibra 2007). However, even if a teacher can help a learner to generalize knowledge, the ability to generalize does not depend on the teacher's cognitive architecture but is intrinsic to that of the learner. We therefore suggest that although theory-of-mind skills can favor the transmission of technical information, the potential to acquire, generalize (i.e., analogical reasoning), and improve it is orthogonal to theory of mind because it is based on technicalreasoning skills.

This leads us to propose the virtuous circle hypothesis of CTC, which is intended to solve the question of how a learner can improve her or his technical-reasoning skills if, during each learning phase, the role of the teacher is minimal (Osiurak et al. 2020a). The idea is as follows. First, models - and teachers - remain fundamental for social transmission. Even if they do not possess outstanding theory-of-mind skills, they still remain a potential source of information, so that everyone can learn even when taught by poor teachers. Second, the role of teachers might be limited to helping learners reach the upper limit of what they can learn in a given situation, an idea very similar to the concept of proximal developmental area (Vygotsky 1978). In the mid-/long-term, this may result in a progressive increase in learners' technicalreasoning skills, thus favoring the emergence of CTC. ${ }^{12}$ Consequently, with regard to the claim that technical expertise is a confound and necessarily depends on previous social transmission (thereby emphasizing the social dimension of CTC), the virtual circle hypothesis holds that: (1) This is certainly right (although not systematic because people can also learn from asocial learning) and (2) without technical reasoning, the progressive accumulation of mechanical knowledge in a single individual via social transmission - i.e., the necessary condition for CTC - could not occur.

\section{Metacognition}

\subsection{Theoretical framework}

Metacognition can be broadly defined as cognition about cognition (Flavell 2000; Shea et al. 2014). Initially, Flavell (1979) distinguished between metacognitive knowledge (i.e., knowledge about variables that can affect the course and outcome of a cognitive activity) and metacognitive experience (i.e., cognitive experiences that accompany and pertain to any cognitive activity). The former includes knowledge about one's own but also others' cognitive capacities, thus establishing a clear link with theory of mind. The latter belongs to the personal level and, as a result, cannot be directly linked to what others can experience - except, perhaps, in the form of a kind of inferential mechanism. More recently, Shea et al. (2014) proposed a revised version of this framework based on two systems referred to as System 1 and System 2 (Heyes 2016; see also Dunstone \& Caldwell 2018). System 1 is very similar to Flavell's concept of metacognitive experience. It does not appear to be specific to humans. System 2 is responsible for forming interpersonal representations about what oneself and others know in a given situation. This second system is hypothesized to be unique to humans (for a similar view, see Gruber et al. 2015), and could differ from theory of mind in that it relates primarily to one's own cognitive processes and is the preferred basis for communication during cooperative activities as well as teaching $^{13}$ (Shea et al. 2014). In this regard, the metacognition hypothesis of CTC suggests that System 2 (simply referred to as metacognition below) provides the main underpinning for sociallearning strategies (i.e., when and whom to copy), providing an adaptive value for humans in terms of CTC (Heyes 2016; 2018). This hypothesis provides an interesting cognitive framework for exploring key questions about social-learning strategies (i.e., transmission biases, flexibility, and overimitation) that we will discuss in turn below.

\subsection{Transmission biases}

Humans live in groups, thus giving them the opportunity to learn from many conspecifics. Evidence shows that group size is critical for CTC in that the presence of a high number of models is beneficial for the stability of a trait as well as for innovation through the combination of solutions produced by the different models (Derex \& Boyd 2015; Derex et al. 2013a; Kemp \& Mesoudi 2014; Muthukrishna et al. 2014). Other evidence has been reported, highlighting an absence of or even an inverse relationship between population size and cumulative performance (Caldwell \& Millen 2010; Collard et al. 2005; 2016; Fay et al. 2019; Vaesen et al. 2016). This discrepancy indicates that deciding whom to copy is a complicated task (Fay et al. 2019). One possibility is that humans copy random things from random people. This form of transmission is called unbiased copying (e.g., individuals have a $60 \%$ probability of copying a trait that $60 \%$ of people possess; Boyd \& Richerson 1985). However, this kind of transmission is not adaptive (Boyd \& Richerson 1985; Giraldeau et al. 2002; Rogers 1988) and does not account satisfactorily for the diffusion of innovation (Rogers 1995). 
Consequently, another more likely possibility is that social transmission is necessarily biased by a certain number of rules, such as the prestige bias ${ }^{14}$ or the conformist bias (Henrich 2001; Henrich \& Gil-White 2001; see also Atkinson et al. 2012; Morgan et al. 2012; Osiurak et al., in press). For a cognitive scientist, the key question is to determine the cognitive skills underlying these kinds of bias. As pointed out by Heyes (2018), interpretations in terms of cognitive processes are rare, because research on social-learning strategies has been dominated by disciplines concerned with what individuals do, not what they think (for a similar viewpoint, see Gruber 2016). Nevertheless, some interpretations have been proposed. For instance, Henrich and Gil-White (2001) suggested that the prestige bias necessarily has its roots in the uniquely human ability of infocopying (a concept similar to imitation). Indeed, emulation leads individuals to focus only on the result and not on the process. The corollary is that if an individual only focuses on the result, she or he is unable to detect, among a number of different models, those who can achieve the expected result the most efficiently (i.e., the prestigious individual). This emphasis on imitation is consistent with the theory-of-mind hypothesis of CTC, even if the purpose differs. Whatever the case may be, this interpretation is difficult to support for the same reasons as evoked above: Nonhumans can exhibit imitation but not CTC. More problematically, the evidence indicates that conformist and prestige biases can also be observed in nonhumans (e.g., Horner et al. 2006; 2010; Kendal et al. 2015; Whiten et al. 2005). Another interpretation is that these biases may favor CTC by allowing humans to learn complex technologies more quickly from their conspecifics via technical reasoning. The distinction between humans and nonhumans would reside more in the complexity of the transmitted technique (see sect. 2.4) than in the kind of social-learning strategies employed (for a similar view, see Whiten et al. 2003).

\subsection{Flexibility}

There are many forms of social-learning strategies that can be distinguished depending on when (e.g., copy-when-uncertain and copy-when-unsatisfied) and whom (e.g., copy-the-majority and copy-successful-individuals, i.e., two strategies that lead to the above-mentioned conformist and prestige biases, respectively) to copy (Kendal et al. 2018; Laland 2004). Models have shown that populations of flexible learners who switch between social and asocial learning at key moments outperform populations of purely social or purely asocial learners (Ehn \& Laland 2012; Enquist et al. 2007; Rendell et al. 2010). In line with this finding, it has been suggested that flexibility not only between social and asocial learning but also in social-learning strategies might be particularly adaptive and critical for CTC (Galef 1995; Heyes 2016; Kendal et al. 2005; 2018; Laland 2004). Henrich and Gil-White (2001) had already suggested that humans are default infocopiers (i.e., imitators) who try first to learn from others instead of "reinventing the wheel" and then seeking improvements via asocial learning. The metacognition hypothesis of CTC posits that this flexibility is based on the ability to form explicit strategies about when and whom to copy (Heyes 2016; 2018; Shea et al. 2014). Even if evidence is still needed to demonstrate that this flexibility is unique to humans (Kendal et al. 2018), this hypothesis is theoretically viable within a limited scope. We say "within a limited scope" because it provides an adequate account of the "when" and "who" strategies. By accessing one's own cognitive activity, an individual can explicitly decide to learn from others because she or he is uncertain or unsatisfied about her or his performance (the "when" strategies). By representing others' cognitive skills, the same individual can also explicitly decide to learn from successful individuals (the "who" strategy). Nevertheless, this hypothesis does not specify the domain of cognition targeted.

Metacognition is cognition about cognition. Therefore, given that we are concerned with technical skills, all these strategies whether explicit or not - are necessarily based on a technical, cognitive content, and it seems most probable that this content takes the form of technical-reasoning skills. To some extent, this is consistent with Henrich and Gil-White's (2001) proposal according to which social-learning strategies cannot be fully effective if individuals cannot accurately represent others' skills. When we observe someone else who is able to fix shelves very quickly by using a series of effective mechanical actions, the prestige we attribute to this individual results from our appraisal of her or his technical-reasoning skills, which differ from our own. In other words, the quality of our metacognitive representations could depend to a very great extent on the content of the targeted knowledge, with the result that the main difference between humans and nonhumans might not relate to metacognition but rather to the quality of the content processed by metacognition (i.e., technical-reasoning skills).

\subsection{Overimitation}

Overimitation refers to the imitation of actions that are causally irrelevant to the achievement of a goal (Lyons et al. 2007; 2011; see also Nagell et al. 1993). This phenomenon has aroused interest in the CTC research community because of the commonly made link between imitation and CTC (Legare \& Nielsen 2015; Shipton \& Nielsen 2015). Evidence indicates a trend toward increasing overimitation with age, with most 2 -year-olds exhibiting emulation, and most 5-year-olds exhibiting overimitation (Horner \& Whiten 2005; McGuigan \& Whiten 2009; McGuigan et al. 2007; Nielsen \& Tomaselli 2010). Overimitation might be even greater in adults (McGuigan 2012; McGuigan et al. 2011; see also Caldwell et al. 2012). A potential interpretation of this phenomenon is that humans may ontogenetically develop specific social-learning strategies, progressively leading them to copy prestigious and competent individuals (i.e., prestige bias; McGuigan 2012). In line with this idea, a significant body of evidence has indicated that children can exhibit two classes of biases, the first being to display a preference for the information provided by a familiar caregiver versus a stranger and the second being to display a preference for someone who has proven to be a reliable source of information in the past (Harris \& Corriveau 2011). The corollary is that children may prefer to learn from individuals who provide reliable information and care, namely parents (Hewlett et al. 2011). However, because their parents may be less accurate and reliable than other individuals, children may progressively opt for more accurate informants, leading to a shift from vertical to oblique or horizontal transmission (Henrich \& Broesch 2011; see also Harris \& Corriveau 2011). This perspective can explain why the aforementioned presence of overimitation found in dyadic interactions (one model: an adult; one learner: a child) tends to disappear in micro-society paradigms in which children interact with one another (e.g., Flynn 2008).

To account for the developmental trajectory of overimitation, McGuigan (2012) suggested that young children may lack the cognitive "sophistication or motivation" needed to overimitate, 
thereby drawing a parallel between the emergence of overimitation and the development of social, cognitive skills. This proposal is consistent with the metacognition hypothesis of CTC in that children may progressively develop social-learning strategies based on their ability to accurately represent what they know, what others know, as well as whether what others know is reliable. However, to be complete, this hypothesis needs to specify the aspect of cognition at which these metacognitive skills are directed. As mentioned above, we assume that metacognitive skills necessarily work in concert with technical-reasoning skills, which are the only appropriate candidate for explaining how an individual can form representations about her or his own and others' technical skills. In other words, social-learning strategies such as the prestige bias cannot be the result of reflecting on cognitive skills in the abstract, but must instead be the result of thought focused on specific cognitive skills. In this perspective, the technical-reasoning hypothesis of CTC offers a revised interpretation of overimitation by stressing that this phenomenon could not occur in the absence of technical-reasoning skills. This hypothesis could be tested in left brain-damaged patients with tool-use disorders. The prediction is that these patients might not exhibit certain social-learning strategies (e.g., a prestige bias) because of their inability to anticipate the effectiveness of their mechanical actions as well as of those performed by others.

\subsection{Interim conclusion}

The metacognition hypothesis of CTC is based on the parallel between the apparently uniquely human ability to exhibit complex metacognition and the fact that CTC is present only in humans. As we have emphasized, this parallel needs to be proven. Nevertheless, this hypothesis offers a viable theoretical framework in which to interpret social-learning strategies, such as the conformist bias or the prestige bias. Nevertheless, it is subject to the same limitations as the theory-of-mind hypothesis. First, it cannot explain why signs of CTC are observed in reverse-engineering conditions in which social transmission is not direct. This observation is inconsistent with the metacognition hypothesis of CTC, which assumes that some strategies can be developed based on the detection of the most frequent variants or the variants proposed by prestigious and competent individuals, namely information that is lacking in reverse-engineering conditions. Second, metacognition is cognition about cognition. However, this hypothesis tends to overlook the domain of cognition in question and therefore offers an incomplete view of how metacognitive skills might operate. In the face of these limitations, we propose a revised interpretation according to which technical-reasoning skills can play a key role by providing humans with useful content for representing not only their own but also others' technical skills. In line with this, we recently demonstrated with a closed-group micro-society paradigm that participants tend to progressively copy the most prestigious individual of the group, that is to say the one with the best technical-reasoning skills (Osiurak et al., in press). In other words, this study shows a direct link between social-learning strategies - and potentially metacognitive skills - and technical-reasoning skills.

\section{Conclusion}

CTC is both a social and technological phenomenon. By focusing on the social dimension, most accounts of CTC have tended to minimize the technical dimension and the potential influence of non-social cognitive skills, perhaps failing to see the elephant in the room. Here, we offer a cognitive view that contrasts with these accounts by assuming that CTC originates in uniquely human technical-reasoning skills. In broad terms, we assume that technical reasoning is a difference-maker that has allowed humans to become cumulative and this to a greater extent than in other species, thereby explaining why this phenomenon is unique to humans. This view acknowledges the important role played by social-learning mechanisms, which are crucial in that they enrich the technical content. As illustrated in our example of someone improving upon Einsteinian physics, social learning is undoubtedly a mechanism through which an incredible amount of information can be transmitted, and the technical solution that is created is novel only up to a point. Nevertheless, humans are not "passive" technical learners because they can also transform the acquired technique into new technical solutions. In this way, the technical-reasoning hypothesis is akin to the cultural attraction hypothesis, which stresses the key role of individual cognition in cultural transmission (e.g., Sperber 1996; Sperber \& Hirschfeld 2004).

The technical-reasoning hypothesis of CTC also does not rule out the potential boosting role played by other cognitive skills, such as theory-of-mind or metacognitive skills. The former might be particularly useful when information is lacking and the latter might help in the selection of reliable models. Nevertheless, they are not necessary conditions for the emergence of CTC. In addition, one outstanding question is whether they should be considered as cognitively distinct or not, given the clear apparent link they share at a theoretical level. They also share other features, such as their close relationships with language skills, also considered as another potential factor of CTC (Pinker 2010; see also Dean et al. 2014; Whiten \& Erdal 2012). Some authors have also stressed potential interactions with future-planning skills (Vale et al. 2012). The question is whether all these cognitive processes are different labels for a single cognitive capacity (or even module), which consists of thinking about oneself or others in different spaces and times. At a phylogenetic level, we can hypothesize that technical-reasoning skills might have played a key role in the development of these skills, a viewpoint that resembles the cognitive niche hypothesis (Pinker 2010; Tooby \& DeVore 1987; see also Osiurak 2017). In other words, the sophistication of hominin technology could have led to cognitive adaptations, such as the emergence of theory-of-mind skills. ${ }^{15}$ However, the small but important difference here is that the level of sophistication of these other cognitive "modules" could not have been reached without technical-reasoning skills.

This leads us to address an overlooked, yet fundamental, question concerning CTC: Why do we continue to improve our technologies? Given that CTC is characterized by constant innovation, research on the topic should attempt to address the question of why we seem to want to constantly improve our technologies. One potential answer is that early hominin tool use could have been developed by necessity in response to environmental pressures (i.e., survival; Boesch \& Tomasello 1998; Wynn 1993). This hypothesis has also been proposed for nonhumans, but has received no empirical support (e.g., Koops et al. 2014; Sanz \& Morgan 2013; but see Gruber 2016). However, this does not explain why we continue to make tools that have no apparent benefits for survival (e.g., TV remote control). ${ }^{16}$

Another answer consists of focusing not on external incentives but rather on the intrinsic characteristics of the cognitive system. In this Lorentzian view, the answer is simple: We constantly improve our tools because we generate our own physical problems 
through technical-reasoning skills. As extensively discussed above, technical-reasoning skills allow us to produce technical solutions that increase our effectiveness and efficiency. In this way, they are a generator of solutions. However, our technical solutions are never fully effective/efficient, even if they can give us the illusion of constantly having to do less. It is not sufficient to think of an environmental effect (e.g., switching on the TV) to make it happen: motor actions are always necessary (pressing a button). There is therefore a gap here between the benefits provided by any technical solution and its ultimate goal (i.e., appearance of an environmental effect without any motor action). This gap represents a self-generated problem space, leading humans to find more effective/efficient solutions, as illustrated by the fantasies regarding teleportation and telekinesis that are present in human cultures (Hubert \& Mauss 1902; see Osiurak 2017; Osiurak et al. 2010). It should be noted that some of these fantasies almost become reality. We can take the train to transport ourselves without moving (teleportation) or use a crane to move objects far too heavy for us (telekinesis). However, the fact that we are far from fulfilling them - and that we will certainly never fulfill them -, provides an increasing room for technical improvement. In this respect, the two sides of technical reasoning (i.e., as a generator of both problems and solutions) form an ideal, theoretical framework in which to understand the emergence and the cognitive origins of CTC in humans.

To conclude, we emphasize the implications of the technicalreasoning hypothesis for our understanding of CTC. To date, this hypothesis has received support from neuropsychological and neuroimaging research. It has recently been applied to the study of CTC by means of micro-society paradigms, which are useful for investigating the role of individuals' cognitive skills in CTC or comparing the quality of social transmission in different social-learning conditions (for a discussion of the strengths and limitations of micro-society paradigms, see Caldwell et al. 2019). These paradigms also allow us to collect empirical data that can be compared to theoretical data generated by modeling approaches to test their predictions. However, they also suffer from the limitation that they do not strictly reflect how technical transmission occurs in everyday life, such as in the case of an apprenticeship, in which the apprentice alternates between periods of social learning and individual learning and in which teaching - and particularly verbal teaching - might play a minor role (Gatewood 1985; see also Wynn \& Coolidge 2014). Therefore, we recommend that future micro-society studies introduce this temporal component (i.e., alternation between social and individual learning) to be more faithful to what really happens in technical transmission situations.

One related point concerns the undoubted interest of the technical-reasoning hypothesis for cognitive archeology or neuroarcheology, two disciplines that aim to investigate stone knapping using experimental psychology or neuroscientific methods, respectively. Some attempts have been made to do this and have emphasized the role of certain parietal structures in stone knapping (Hecht et al. 2015; Stout \& Chaminade 2007; Stout et al. 2011; see also Stout \& Hecht 2017). This research is valuable because of the groundbreaking nature of its methodology. However, it does not provide a model of technical cognition that can be applied to other disciplines (Wynn et al. 2017), in particular because it originates in classical neuropsychological models that ignore the important contribution of reasoning in tool behavior (see sect. 2.1). In this respect, the technicalreasoning hypothesis offers an original theoretical framework that can contribute to our insight into the importance of the understanding of functional parameters (i.e., physical principles) in stone knapping when compared to motor procedures (e.g., Bril et al. 2010).

Finally, we have to acknowledge that the technical-reasoning hypothesis is still a new hypothesis, which has been developed by only a few researchers. This is surprising if we consider how dramatically our technical skills have modified the surface of the Earth. Nevertheless, well-known handbooks of cognitive psychology or the cognitive sciences contain no sections on technical cognition. Considerable effort is therefore needed to develop a comprehensive, computational model that could be useful for modeling work, and in particular for testing the predictions of this hypothesis versus those derived from other hypotheses of CTC.

\section{Notes}

1 Mesoudi and Thornton (2018) recently proposed a more dynamic description of CTC, suggesting that the minimal requirements for a population to exhibit CTC are: innovation (based on asocial learning), imitation (based on social learning), improvement in performance at the group level (cultural fitness), and repetition of the previous three steps (improvement over time).

2 Social learning refers to learning that is influenced by observation of, or interaction with, another individual (typically a conspecific) or its product (Galef 1988; Heyes 1994). Individual learning refers to individual "practice" periods that occur in between social-learning periods. By contrast, asocial learning refers to behavior that is learned individually without being the consequence of social learning.

3 Povinelli and Frey (2016) conducted a series of experiments in which they presented chimpanzees with two visually identical rakes. Only one rake was functional (i.e., rigid). They explored whether chimpanzees manually tested the rake's rigidity before using it. They found no evidence for such behavior, suggesting that chimpanzees do not exhibit prospective diagnostic skills. Martin-Ordas et al. (2008) investigated the performance of apes (chimpanzees, bonobos, orangutans, and gorillas) on two versions of the trap-tube task. In this task, animals are presented with a transparent tube from which they have to extract a reward by using a stick, without pushing the reward inside a trap located in the center of the tube. There was no correlation in the level of performance between the two tasks, suggesting an absence of transfer between functionally equivalent tasks. Taken together, these findings demonstrate that nonhuman primates have difficulty understanding unobservable causal properties.

4 For the sake of clarity, we will use hereafter the term technical reasoning even if authors have used the term causal reasoning in their original papers. 5 In a way, all imitation is emulative, as suggested by Ingold (1998).

6 In line with this idea, we found that left brain-damaged patients with technical-reasoning disorders exhibit severe difficulties in initiating any strategy that includes trial and error to solve mechanical problems (Osiurak et al. 2013). By contrast, even healthy controls with poor technical-reasoning skills have been found to be able to follow trial-and-error strategies, leading them to use irrelevant tools to interact with the problems, and sometimes to find the solution (i.e., reasoned trial and error). Their solutions were not complete but at least partially complete, so that the interactions enabled them to improve the initially generated solutions. Finally, only healthy controls with good technical-reasoning skills spontaneously selected the appropriate tools to perform the intended mechanical actions (i.e., full understanding of the task; for additional evidence in Alzheimer's disease and semantic dementia, see Lesourd et al. 2016).

7 Creativity - and particularly its divergent-thinking dimension - is thought of as the key ability in the production of new and original ideas (Guilford 1967). The link between innovation and creativity is straightforward. We recently explored this link by assessing the divergent-thinking skills of participants in a micro-society paradigm (De Oliveira et al. 2019). Participants' technical-reasoning skills were also measured. As mentioned above, participants' technical-reasoning skills were the best predictor of cumulative 
performance. By contrast, divergent-thinking skills were negatively linked to cumulative performance, suggesting that participants with high creativity tended to produce ineffective productions. This finding suggests that innovation is based on technical expertise, corroborating previous work on creativity that has shown that knowledge is one of the most important factors of creativity (Simonton 2000). In sum, the hypothesis that technical reasoning supports innovation is somewhat counterintuitive because it stresses that expertise matters much more in innovation than talent.

8 However, the presence of CTC in this study remains to be demonstrated given the absence of an asocial-learning control condition in which a single participant performed the 25 trials instead of five participants performing five trials each (Mesoudi \& Whiten 2008).

9 The development of theory-of-mind skills during childhood is not binary (i.e., presence vs. absence) but follows a series of steps that have been well documented by Tomasello and colleagues (Tomasello et al. 1993; 2005). For this reason, Tomasello and colleagues preferred not to use the term theory of mind, which can refer to this binary dimension. Here, we will nevertheless use the generic term of theory of mind.

10 The theory-of-mind hypothesis of CTC posits that humans and some great apes might possess relatively similar cognitive skills for dealing with the physical world, but not the social world. Support for this idea comes, for instance, from the study by Herrmann et al. (2007), who gave a battery of physical (i.e., space, quantities, and causality) and social (i.e., social learning, communication, and theory of mind) cognitive tests to 2.5 -year-old children and great apes (i.e., chimpanzees and orangutans). Taken as a whole, findings indicated that children outperformed apes only on social, cognitive tasks. However, a thorough scrutinization of the data reveals that children were better than great apes on three of the four causality tests, and particularly a test investigating the understanding of the physical properties of tools (e.g., selecting between a stick cut in two pieces vs. an intact stick to retrieve an out-of-reach object/food). The only causality test in which chimpanzees (but not orangutans) outperformed children was a tool manipulation test, consisting of using a stick to retrieve an out-of-reach object/food. This was also the only test in the battery with only one trial. Indeed, the authors themselves acknowledged in this study that 2.5 -year-old children might be more skillful than the apes in causality tests, but interpreted these findings as the potential generalization of theory-of-mind skills to the physical domain.

11 The opaque/transparent nature of an artifact should undoubtedly be thought of as a continuous and not a dichotomic variable. However, for the sake of clarity, we will consider it as dichotomic.

12 This virtuous circle can occur, in particular, when a single teacher accompanies a learner over time. The micro-society paradigms commonly used to investigate CTC might not be appropriate for testing this virtuous circle hypothesis, because social interactions are generally limited (i.e., a single interaction). A more appropriate design might be to propose repeated interactions between a teacher and a learner separated by individual-learning periods. This could help demonstrate that teachers' theory-of-mind skills play an increasingly important role in social transmission over time.

13 We will not address here the link between metacognition and teaching because it is very similar to the above discussion about theory of mind and teaching. 14 Henrich and Gil-White (2001) suggested that the formation of prestige hierarchies could have emerged from the copy-successful-individuals strategy. Individuals following this strategy may be selected to pay deference to successful individuals in exchange for assistance and access in learning. Interestingly, this deference behavior is a reliable clue as to which individuals possess adaptive knowledge (Laland 2004). Given that we will not discuss this deference behavior in detail, we will use the terms prestigious and successful (particularly in the technical domain) interchangeably.

15 Gergely and Csibra (2006) proposed a similar view, suggesting that the sophistication of technology has progressively led to the building of artifacts whose construction has become opaque for most individuals in the group. This might therefore have provoked the emergence of "pedagogical" skills to allow humans to transmit technical information even in the case of sophisticated artifacts (see the author's just-so-story).

16 In line with this idea, recent evidence shows that humans prefer to use tools even when this is less effective than doing without them (Osiurak et al. 2014; Virgo et al. 2017), a bias that has not been found in nonhuman tool-using species (e.g., New Caledonian crows; Danel et al. 2017).
Acknowledgments. We thank Tim Pownall for the English proofreading of this manuscript. This work was supported by grants from ANR (Agence Nationale pour la Recherche; Project "Cognition and tool-use economy" ECOTOOL; ANR-14-CE30-0015-01), and was performed within the framework of the LABEX CORTEX (ANR-11-LABX-0042) of Universite de Lyon, within the program "Investissements d'Avenir" (ANR-11-IDEX-0007) operated by the French National Research Agency (ANR).

\section{Open Peer Commentary}

\section{Implications for technological reserve development in advancing age, cognitive impairment, and dementia}

\section{Jared F. Benge ${ }^{a}$ and Michael K. Scullin ${ }^{b}$ e}

${ }^{\mathrm{a} D e p a r t m e n t}$ of Neurology and Plummer Movement Disorders Center, Baylor Scott and White Health, Texas A\&M College of Medicine, Temple, TX 76508 and ${ }^{\mathrm{b}}$ Department of Psychology and Neuroscience, Baylor University, Waco, TX 76798.

Jared.Benge@BSWHealth.org

Michael_Scullin@Baylor.edu

sites.baylor.edu/scullin

\section{doi:10.1017/S0140525X20000126, e157}

\section{Abstract}

This commentary draws connections between technological culture emergence and recent trends in using assistive technology to reduce the burden of Alzheimer's disease. By the technicalreasoning hypothesis, cognitively-impaired individuals will lack the cognitive ability to employ technologies. By the technological reserve hypothesis, social-motivational and cultural transmissibility factors can provide foundations for using technology as cognitive prosthetics even during neurodegenerative illnesses.

According to Osiurak and Reynaud (O\&R), cognitive rather than social mechanisms lead to technological culture emergence. Their theory, which is focused on psychological and socio-cultural explanations for technological culture development, also happens to come at a critical time for addressing the real-world burden of Alzheimer's disease and related dementias.

It is estimated that by the year 2050, as many as 152 million individuals across the world will be suffering from dementia (Alzheimer's Disease International 2018), causing substantial impairments to daily life. For example, persons with dementia may be unable to live safely alone, travel within their community, or manage medications and finances. Each of these impairments requires a concomitant increase in effort by informal caregivers (typically, the spouse or child), a burden that comes at the cost of caregivers' physical and mental health (e.g., Gao et al. 2019). However, at the same time as dementia prevalence and burden are increasing, humanity's cumulative technological culture is producing innovative technologies that may help individuals compensate for their impairments. Self-driving cars and ride share apps might allow persons with dementia to travel relatively 
independently even when driving restrictions are in place; video messaging and social media may relieve feelings of social isolation and depression; smartphone devices can provide reminders for prospective memory tasks such as taking medications and paying bills; and smart home technology and wearables can monitor the patient to mitigate safety issues such as falls and wandering (Benge et al. 2020; Neves et al. 2019).

O\&R's theory on technological culture is thus timely for considering a concept we have termed technological reserve. Technological reserve refers to the development of a culture and environment of technology use in older adults that can buffer against the impact of cognitive decline on day to day activities. In a very tangible way, a technologically-rich environment and culture may lessen the impact of neuropathological changes on day to day activities for those afflicted with neurodegenerative conditions, even without directly altering the disease itself. Though O\&R's theory was intended to explain the development of cumulative technological culture as a whole, at the level of the individual, their theory provides a framework for understanding both the challenges and the opportunities for technology and dementia research.

O\&R's theory provides an explanation for the phenomenon of reduced technology use with aging and age-related cognitive decline (Span et al. 2013). Some have argued that social/attitudinal processes are core to engaging older adults in technological culture (Charness \& Boot 2009). O\&R though provided a stark contrasting perspective that cognitive rather than social mechanisms impact technological culture development, a view that we believe explains trends in the technology and dementia literature. For example, although social skills tend not to be grossly impaired with normal aging - or even with cognitively-impairing conditions - basic executive processes decline and this decline is associated with a decreased use of common everyday technologies (Wu et al. 2019). Furthermore, older adults who have greater levels of cognitive impairment show less use (but not zero use) of the breadth of digital technology available to them (Czaja et al. 2006). By this interpretation of their theory, cognitive decline will relentlessly undermine technical reasoning, thereby leading to futility in using assistive technology to improve the lives of persons with dementia.

However, there is another, perhaps more optimistic, possibility. As the authors noted, cumulative technological culture is transmissible. This implies that as technologically-savvy cohorts age (i.e., people with early and long-instilled experience and motor memories for smart systems), their established technology habits can provide jumping off points for rehabilitative strategies even in the midst of cognitive decline. In this way, vestiges of technical skills may exist as their disease progresses, which can be leveraged into technology-based compensatory tools in clinical settings. After all, the field of cognitive rehabilitation has long noted that the most effective compensatory strategies tend to build on skills and habits previously attained by the patient (Sohlberg \& Mateer 2017).

We need not wait though for the most technology-savvy cohorts to reach older age to begin testing the concept of technological reserve (Lee et al. 2019). Two critical points have already emerged on how to foster and optimize technology use for persons impacted by neurodegenerative diseases. The first is evidence that patients with cognitive disorders and their care partners spontaneously report using the compensatory features on their smartphones more than demographically-matched peers (Benge et al. 2020). Thus, in the face of progressive cognitive decline, individuals are already reaching toward technology to help them compensate. Second, opportunities for social networking may serve as an incentive for older adults to engage with digital technology on a daily basis. Approximately half of patients report using email every day (Benge et al. 2020) and 30-60\% of older adults have a social media account (Pew Research Center 2018). These social features of technology help to reinforce engagement with devices and cognitive-assisting systems. These positive trajectories signal the development of a subculture in the aged that remains technologically-engaged even in the face of cognitive decline.

O\&R's astutely noted that people are "generators of solutions." As persons with dementia, their caregivers, and the broader research field struggle with the oncoming wave of neurodegenerative illness, we are in need of humanity's technical skills now more than ever. There is an urgent need to understand how to capitalize on technical-reasoning skills to build an environment where individuals and their care partners can live well, even in the face of diseases that rob the skills that gave rise to technological culture to begin with.

Financial support. This work was supported by the National Institutes of Health (AG053161).

Conflict of interest. None.

\section{Chimpanzees' technical reasoning: Taking fieldwork and ontogeny seriously}

\section{Christophe Boesch $(0)$}

Max Planck Institute for Evolutionary Anthropology, 04103 Leipzig, Germany. boesch@eva.mpg.de

https://www.eva.mpg.de/primat/staff/boesch/index.html

\section{doi:10.1017/S0140525X2000028X, e158}

\section{Abstract}

Following the tradition of comparing humans with chimpanzees placed under unfavorable conditions, the authors suggest many uniquely human technological abilities. However, chimpanzees use spontaneously tools in nature to achieve many different goals demonstrating technological skills and reasoning contradicting the authors contrast. Chimpanzees and humans develop skills through the experiences faced during their upbringing and neglecting this leads to fake conclusions.

The authors in their review of technological culture are proposing a series of unique human abilities that seem to distinguish humans from other animals. By doing this, they are following an unfortunate tendency to erect "Golden Barriers," as said Stephen Jay Gould, to set us apart from the rest of the animal kingdom. This is commonly done with a simple trick: Namely, place the species compared with humans under unfavorable conditions (Allen 2002; Bard \& Leavens 2014; Rowe \& Healy 2014). The result being that humans always fare better. Thus, the real "elephant in the room" is if this is science! 


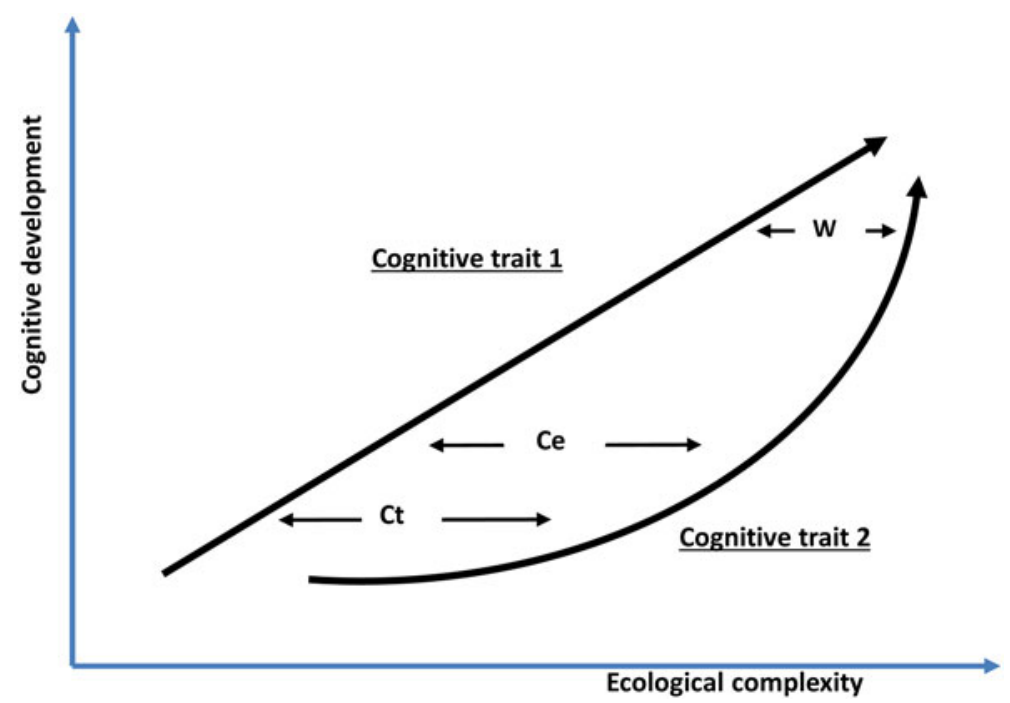

Figure 1 (Boesch). The eco-cultural model for cognitive development: Four main factors have been shown to affect the development of cognition in animals, including age, ecological complexity, social, and cultural complexity. The potential effects of ecological complexity on two different cognitive traits when animals develop in traditional captive conditions $(\mathrm{Ct})$, in environmental enriched captive conditions (Ce), or in the wild (W) are shown.
In their otherwise thorough review, the authors base all their comparative arguments on a few captive chimpanzee studies. Whenever they mention results on wild chimpanzees they regularly contradict them with captive experimental studies to which they give priority. Why is such a comparison unfair?

First, all leading hypotheses about the evolution of cognition have proposed the importance of ontogeny and socio-ecological factors. This is true for the ecological-intelligence hypothesis (Byrne 1997; Clutton-Brock \& Harvey 1980), the social intelligence (Dunbar 1992; Humphrey 1976), the cultural intelligence (Byrne \& Whiten 1989), as well as the eco-cultural hypotheses (Boesch 2007; Carpendale \& Lewis 2004; Segall et al. 1999). Second, all studies evaluating the effects on cognition of the physical or social environment, as well as of experience, agreed to conclude that all these factors have a measurable positive effect on its development (e.g., Bard \& Leavens 2014; Buchanan et al. 2013; Fares et al. 2013; Hackman \& Farah 2009; Lambourne \& Tomporowski 2010; Leeuwen et al. 2014; Middleton et al. 2008; Noble et al. 2015; Pike et al. 2018; Russell et al. 2011; Sallet et al. 2011; Salvanes et al. 2013; Thornton \& Lukas 2012; Toyoshima et al. 2018).

Figure 1 illustrates how two different cognitive traits, such as technical skills (trait 1, Sirianni et al. 2015; 2018) or spatial knowledge (trait 2, Normand et al. 2009), could be affected by the ecological complexity in which the individual lives. The strongly limited access to objects in captivity has been shown to reduce the development of an understanding of the function of tools that could partially be improved with increased access to tools (see Bania et al. 2009; Furlong et al. 2008), and will further be favored in the wild where tools are of a real value (Sirianni et al. 2015; 2018). Although, spatial skills will remain very limited in captivity because of the strong space restriction of the environment (Premack \& Premack 1983), they can develop in the wild when foraging in kilometers-wide territories (Normand \& Boesch 2009).

The chimpanzees studied by Povinelli and Tomasello cited extensively by the authors have for the first had only very limited access to objects in an artificial small peer-group without any adult models, whereas the second ones started in a medical research center subject to invasive experiments before experiencing better living conditions at the Leipzig zoo. As could have been expected both groups of chimpanzees show striking deficiencies in their understanding of tool function and integrity, notion of weight, space as well as in the social cognition, like the absence of cooperation and imitations (reviewed in Povinelli 2000; 2012; Tomasello 2019b). Basing themselves on such human-shaped chimpanzees and rejecting field observations if they disagree with the formers, the authors built some nice dichotomy endorsing so-called human superiorities.

How would a fair comparison look like? What if free-ranging socially natural living chimpanzees were compared with humans living under similar conditions? In this way, natural differences would be identified to understand the evolution of human uniqueness. To restrict myself to technology, all chimpanzee groups studied throughout Africa use flexibly extended tool kits and often fashion the tools before use. Furthermore, they often plan the future needs of tools resulting in tool transports, with precise selection and/or modification of tools as a function of distance transported (Boesch \& Boesch 1983; 1984; 1990; Boesch et al. 2009; Estienne et al. 2019a; 2019b; Goodall 1968; 1970; Luncz et al. 2012; Sanz \& Morgan 2007; 2009; Sanz et al. 2004; 2009; Sirianni et al. 2015; 2018; Sugiyama 1994; Sugiyama \& Koman 1979). Mothers teach youngsters to stimulate the learning of tools in different populations of chimpanzees (Boesch 1991; Boesch et al. 2019; Musgrave et al. 2016; 2020). None of these complex characteristics of tool use have been found in captive chimpanzees. Just one example, Taï chimpanzees select an optimal hammer out of an average of 16 available ones by considering five factors, including weight, hardness, distance to the anvil, and nut species, just by looking at them (Boesch \& Boesch 1984; Sirianni et al. 2015) and this selection is condition-dependent on the distance to the nut-cracking site. This requires a complex form of conditional reasoning combining visible and imperceptible properties of the objects. New selections are repeated in numerous new situations throughout the 5 months of the two nut-cracking seasons (Sirianni et al. 2015). Sure, in the forest, tools contribute to the survival of the animals, which is never the case in a captive environment. The value of captive experiments without any ecological validity has been amply critically discussed in the literature (e.g., Boesch 2012; Rowe and Healy 2014; Smulders et al. 2010), but the authors seem not aware of this. 
Comparisons with ecological and ontogenic validity would lead to totally different results than the target paper. As a reviewer of the paper, I informed the authors about the problem, but they were allowed by the journal to ignore this and perpetuating the simple trick to make humans look superior.

Financial support. This research received no specific grant from any funding agency, commercial, or not-for-profit sectors.

Conflict of interest. None.

\section{A cognitive developmental approach is essential to understanding cumulative technological culture}

\section{Emily Rachel Reed Burdett ${ }^{\mathrm{a}}$ (1) and Samuel Ronfard ${ }^{\mathrm{b}}$ ()}

a University of Nottingham, School of Psychology, Nottingham NG7 2RD, UK and

b University of Toronto, Psychology UTMCCT4059, Mississauga, Ontario L5L1C6, Canada.

Emily.burdett@nottingham.ac.uk

samuel.ronfard@utoronto.ca

https://www.nottingham.ac.uk/psychology/people/emily.burdett

https://www.utm.utoronto.ca/psychology/faculty-staff/ronfard-samuel

doi:10.1017/S0140525X20000175, e159

\section{Abstract}

Osiurak and Reynaud argue that children are not a good methodological choice to examine cumulative technological culture (CTC). However, the paper ignores other current work that suggests that young children do display some aspects of creative problem-solving. We argue that using multiple methodologies and examining how technical-reasoning develops in children will provide crucial support for a cognitive approach to CTC.

Osiurak and Reynaud (O\&R) claim that children do not possess the technological expertise required to innovate new solutions to problems and conclude it is debatable whether children are a good "methodological choice" to examine cumulative technological culture (CTC) (section 3.4). Indeed, children do struggle with tasks requiring creative problem-solving and we agree that sufficient technical-reasoning is required for innovation. However, the paper ignores a growing body of research that suggests some early innovative capacities and does not give adequate discussion to the early development of technical-reasoning skills. Indeed, based on recent developmental evidence, we argue that young children display some aspects of creative problem solving under limited conditions. Understanding these constraints on innovation in early childhood is key to understanding what is developing.

O\&R argue based on four developmental studies (Beck et al. 2014; Cutting et al. 2014; Reindle \& Tennie 2018; Reindle et al. 2017) that young children are poor innovators. It is true that young children's innovation is limited when they have to innovate over a short period of time (Beck et al. 2014; Cutting et al. 2014) and with limited materials (e.g., a pipecleaner and string, or water and cup) (Beck et al. 2014; Cutting et al. 2014; 2019; Ebel et al.
2019). However, young children can innovate new and effective solutions when working in small groups (McGuigan et al. 2017), when they have prior experience with the task (Whalley et al. 2017), when the task is open-ended and allows them to use multiple manufacturing methods (i.e., reshaping, adding, subtracting, and detaching) (McGuigan et al. 2017; Voigt et al. 2019), and when they have plenty of time (McGuigan et al. 2017; Voigt et al. 2019). In sum, young children appear to be able to explore their way to a solution but seem restricted in their ability to come up with the "right" solution in tasks that are more constrained both in terms of time, materials, and manufacturing methods (e.g., Beck et al. 2014).

Intriguingly, a similar pattern has been observed when examining the development of children's hypothesis testing. When faced with a surprising event or with surprising data, young children deploy sophisticated exploration and search strategies, make appropriate inferences, and test these hypotheses (e.g., Gopnik 2012; Gopnik et al. 2015; 2017). However, children struggle until middle childhood (and even adulthood in some contexts) to design controlled experiments that isolate causal factors (Chen \& Klahr 1999). Explicitly testing a hypothesis and solving a specific technical problem are analogous in important ways and children seem to solve both tasks around the same time. Around 8-years-old, their problem solving in both contexts is more flexible and targeted and less reliant on imitation and exploration (Chen \& Klahr 1999; Carr et al. 2016; Lucas et al. 2017). Given the cognitive overlap between designing an experiment and developing an innovative solution to a technical problem, the fact that scientific problem solving and innovation follow similar developmental trajectories suggests that domaingeneral developments (in addition to domain-specific knowledge) may play an important role in constraining innovation in childhood.

Some domain-general factors presumed to increase technological reasoning can be tentatively ruled out. On more constrained tasks (like the hook task) executive functioning (Chappell et al. 2013) including inhibitory demands, working memory, attentional flexibility (Beck et al. 2016), and divergent thinking (Beck et al. 2016) are not associated with innovation success rates. By implication, young children are not failing to innovate because of limits in their abilities to process information. Instead, their ability to innovate may be constrained by their ability to make connections between their prior knowledge and current tasks' constraints (analogical reasoning, e.g., Gentner et al. 2016), by their ability to consider how different steps could be taken to solve a problem (advanced planning, Tecwyn et al. 2014), and by improvements in children's metacognition - their ability to represent their own technical skills. This latter skill may be particularly important in allowing children to engage in more targeted forms of innovation and thus may allow children to not only explore their way to innovation but also to direct their way to innovation (see Carr et al. 2016 for a similar proposal).

In conclusion, we agree with O\&R that a suite of non-social cognitive factors contribute to technological reasoning and innovative thinking. We think that more work into understanding the development of these cognitive factors in children is promising. Specifically, we propose that further work examines the development of cognitive factors in both open-ended and constrained tasks. The cognitive skills required for either task may reveal multiple developmental pathways to innovation, such as via exploration or through a more directive, analogical approach. 
Financial support. This research received no specific grant from any funding agency, commercial, or not-for-profit sectors.

Conflict of interest. None.

\section{Taking into account the wider evolutionary context of cumulative cultural evolution}

\section{Nicolas Claidière (i)}

Aix Marseille Université, CNRS, LPC UMR 7290, 13331 Marseille, France. nicolas.claidiere@normalesup.org

http://www.nicolas.claidiere.fr

doi:10.1017/S0140525X20000254, e160

\section{Abstract}

The target article reviews evidence showing that technological reasoning is crucial to cumulative technological culture but it fails to discuss the implications for the emergence of cumulative cultural evolution (CCE) in general. The target article supports the social view of CCE against the more ecological alternative and suggests that CCE appears when specialised individuallearning mechanisms evolve.

The target article offers a refreshing view on the question of the origin of cumulative technological culture (CTC) by providing evidence that CTC crucially depends on technological reasoning and not so much on developed social-learning capacities. However, the authors did not make explicit the wider evolutionary consequences implied by their discussion. The origin of cumulative cultural evolution (CCE) has been widely debated and the subject of renewed research efforts, leading to two broad categories of theories, namely the ecological and the social views of CCE. Let me outline them here briefly.

Under the ecological view, humans' ancestors faced strong selection pressure, potentially in the form of large and frequent climatic changes (Richerson \& Boyd 2005), to adapt to diverse environments. This selection pressure resulted in the evolution of enhanced technological-reasoning skills but also in more tolerant societies based on cooperation and more developed sociallearning mechanisms (e.g., imitation, teaching, and language) that were necessary to acquire the skills to survive in these environments. Crucially, although the ecological view recognises the importance of technological reasoning, the adaptation to varied environments (spatially and/or temporally variable) is realised when technologies can spread and evolve through cultural transmission (Boyd 2013). According to the ecological hypothesis, CCE evolved in order to acquire new adapted behaviour rapidly and efficiently, leading to the emergence of tools and technologies that were beyond what a single individual could produce. Under the ecological hypothesis then, CTC is the driving force behind our success as a species capable of surviving almost any environment and it is the source and the consequence of dedicated social-learning mechanisms such as our unique communication system based on language. This view receives support, in particular, from experiments showing that more complex forms of communication are essential to transmit complex technological skills (e.g., Dean et al. 2012; Derex et al. 2019; Morgan et al. 2015) and from modelling showing that cultural transmission can evolve in certain unpredictable environments (e.g., Rendell et al. 2010).

In contrast, the social view emphasises the importance and complexity of human and non-human primate social relationships (I am lumping here together the social brain hypothesis [Dunbar 1992; 2012] and the Machiavellian-social-cultural intelligence hypotheses [Byrne \& Whiten 1988; Whiten \& van Schaik 2007)]. According to the social view, under, maybe the same, pression from the environment, humans evolved larger groups with more relaxed social relationships based on cooperation and coordination but also more complex forms of relationships and ways of communicating about them, giving rise to theory of mind and language. Under this scenario, social learning in humans evolved for "gossiping," that is, for dealing with relationships in our large hierarchical groups. For the social view, CTC is a by-product of the evolution of social learning: technological reasoning evolved independently to solve concrete problems, and cultural transmission did not evolve to spread technological knowledge. Rather, the evolution of social-learning capacities and a more relaxed sociality evolved for social reasons and created an environment in which technology became cumulative, as did many other behaviours in other social and nonsocial domains. The social view stresses the complexification of social cognition and social relationships among primates and the natural continuity between communication in non-human primates, used mainly to deal with social relationships, and theory of mind and language in humans.

Is CTC the driving force behind the emergence of complex forms of social learning? Or is it a by-product of the evolution of social communication? The target article reviews evidence showing that (i) technological reasoning is critical to produce CTC, (ii) technological reasoning is a specialised cognitive mechanism in humans, and that (iii) minimal forms of social learning (such as the exposure to the products of other individuals) are sufficient to generate CTC. And although one may criticise the difficulty of the experimental tasks (e.g., building spaghetti towers, paper planes, and so on) used to test technological reasoning, it is noteworthy that these simple tasks are already well beyond what non-human primates can achieve. The target article's conclusion, that a difference in technological reasoning is at the origin of the absence of CTC in non-human primates, not a difference in social-learning mechanisms, therefore support the social view of CCE. Furthermore, the fact that complex forms of social learning do not seem to be necessary to produce CCE, combined to the observation that CCE in non-human animals are in domains in which they excel at learning (travelling routes for pigeons [Sasaki \& Biro 2017], songs in birds [Feher et al. 2009], memory task of baboons [Claidière et al. 2014b], for instance), could suggest that CCE appears when specialised individual-learning mechanisms evolve.

Financial support. This research received no specific grant from any funding agency, commercial, or not-for-profit sectors.

Conflict of interest. None. 


\section{The blind men and the elephant: What is missing cognitively in the study of cumulative technological evolution}

\section{Bernard J. Crespi (i)}

Department of Biological Sciences, Simon Fraser University, Burnaby, British Columbia V5A 1S6, Canada.

crespi@sfu.ca

https://www.sfu.ca/biology/faculty/crespi/

doi:10.1017/S0140525X20000151, e161

\begin{abstract}
I describe and explain (1) evidence regarding a key role for autism spectrum cognition in human technology; (2) tradeoffs of autistic cognition with social skills; and (3) a model of how cumulative technological culture evolves. This model involves positive feedback whereby increased technical complexity selects for enhanced social learning of mechanistic concepts and skills, leading to further advances in technology.
\end{abstract}

For a person on the autism spectrum, our elephantine world is populated by sensations, systems, and objects, including other humans considered as mechanistic "things" rather than animate, mindful subjects. For a person on the psychotic spectrum, by contrast, lifeless things become animated with human thoughts and intentions, whereas other humans induce paranoia through mentalistic imagining run amok. Both of these conditions represent extremes. But of what?

I argue here that the evolution of human cumulative technological culture has been driven by synergism of asocial, mechanistic, systemizing cognition, epitomized by autism spectrum traits, with social, mentalizing, imaginative cognition, epitomized in the extreme by psychosis. This argument represents an extension, elaboration, and constructive criticism of Osiurak and Reynaud's (O\&R's) suggestion that human technicalreasoning skills have been crucial for cumulative technological culture.

I first describe the evidence that autism spectrum cognition is associated with engineering, physics, and mathematics, whereas psychotic spectrum cognition is associated with social imagination, the arts, and the humanities. In this context, I also explain how and why traits on these two spectra tend to trade off with one another, such that enhanced skills in one domain involve reduced abilities in the other. Second, I present a model whereby synergism between mentalistic and mechanistic cognition and skills drives technological complexification though positive feedback.

Evidence for positive associations of the autism spectrum with STEM disciplines comes from several independent lines of inquiry. Epidemiological studies have thus shown that persons with professions in, or persons who plan to enter, STEM areas show relatively high rates of autism among their relatives, compared to controls (Baron-Cohen 1998; Baron-Cohen et al. 1997; 2007; Campbell \& Wang 2012; Roelfsema et al. 2012; Spek \&
Velderman 2013; Wei et al. 2013; Windham et al. 2009). By contrast, psychotic-affective disorders are linked with professions and abilities in the arts and humanities (Kyaga et al. 2011; Nettle 2006; review in Crespi et al. 2016).

Physical-engineering reasoning abilities, which align with O\&R's conception of technical skills, have been quantified using the Intuitive Physics Test (Baron-Cohen et al. 2001). Scores on this task are higher among individuals with ASD than in controls (Baron-Cohen et al. 2001; Binnie \& Williams 2003; Jack \& Gabriel 2013), and such individuals also show stronger "explanatory drive" regarding physical systems than do controls (Rutherford \& Subiaul 2016).

These findings, coupled with relatively high fluid (compared to crystallized) intelligence (Hayashi et al. 2008) and high "systemizing" cognition (Baron-Cohen et al. 2011) in autism, positive genetic correlations of autism risk with general intelligence (reviewed in Crespi 2016), and the mechanistic nature of autistic savant skills (Happé \& Frith 2010), indicate that "highfunctioning" autism spectrum cognition is closely aligned with human technical abilities. Indeed, autism is the only psychiatricpsychological condition and variation to do so, to my knowledge. So might individuals or groups with relatively more autismrelated traits have driven cumulative human technological change?

Probably not, because technical expertise usually comes with costs in social skills that would stymie social transmission. Indeed, "folk physics" shows clear evidence of tradeoffs with "folk psychology" (Baron-Cohen 2000; Baron-Cohen et al. 2001), technical and visual-spatial skills trade off with social abilities more generally in autistic as well as non-autistic individuals (Crespi \& Go 2015, Table 1), and autism is well known to engender reductions in ability and/or motivation to imitate, emulate, innovate, engage in joint attention, and, most broadly, enculturate (e.g., Baron-Cohen 1993; Charman 2003; Colombi et al. 2009). The existence of such tradeoffs vitiates the direct connection of technical reasoning with imitation and innovation posited by $\mathrm{O} \& \mathrm{R}$ as key to their model. The neurological sources of mechanistic-mentalistic tradeoffs appear to stem in part from the anticorrelated nature of activation of the task-negative, default, social, "imagination" brain activation mode with the task-positive, problem-solving, mechanistic, "intelligence-associated" mode, such that these two systems are incompatible (Jack et al. 2013), and individuals (and the two sexes; Baron-Cohen et al. 2011) specialize in one cognitive domain at each point in time. Cognitive tradeoffs are, crucially, missing from O\&R's conceptual scheme. How might they be incorporated?

Synergism between technical and social abilities provides a useful model for the evolution of cumulative technological culture. By this paradigm (Fig. 1), mechanistic and mentalistic abilities complement one another within humans and in human groups of sizes from two to many. Increases in human technical skills, as may follow from greater fluid intelligence, complexify and diversify human technology. These changes, in turn, generate natural selection for better social-learning and socialcommunication abilities, to transfer the improved technology via teaching. Enhanced social learning, and enhanced technology development, can thus coevolve via a positive feedback loop, through synergisms between mechanistic and mentalistic thought. Conceptually comparable positive feedbacks have been proposed to explain many of the other major transitions in ecology, evolution, and behavior (Crespi 2004). 


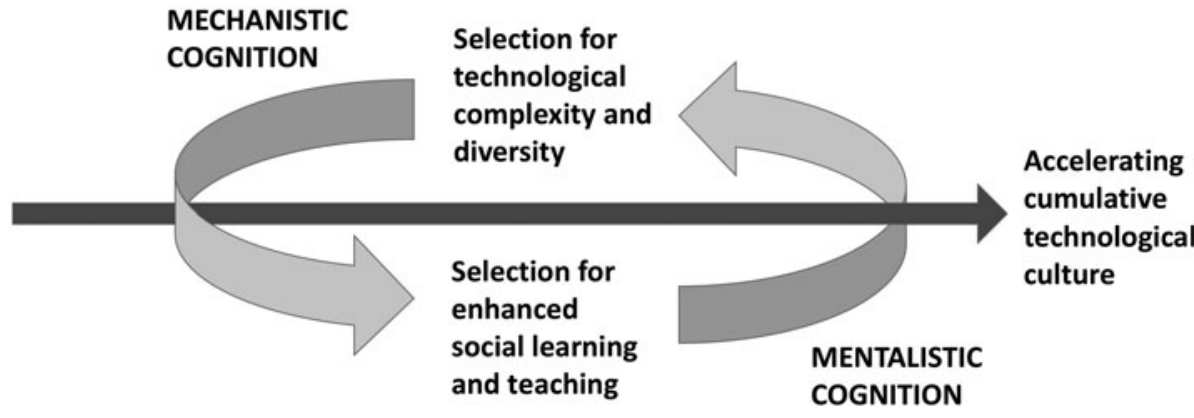

Figure 1 (Crespi). Model for the cognitive evolution of cumulative technological culture.
The synergism model is supported by: (1) a recent study showing that social learning in young chimpanzees is more highly scaffolded and structured in a more-technologically advanced chimp population (Musgrave et al. 2020; Whiten 2020); (2) the human micro-society studies that show enhancement of technology transfer when technical and theory of mind skills are combined (e.g., Osiurak et al. 2020a); (3) a body of work showing that combinations of autistic-like and schizophrenia-like traits can optimize task performance (e.g., Abu-Akel et al. 2015); and (4) recent cases of technological change in human societies, such as the synergism between Steve Wozniak, who showed many Asperger-like traits (Wozniak 2007) and Steve Jobs, who has been ascribed a suite of borderline and narcissistic traits, as well as a tendency toward "reality distortion" more generally (Isaacson 2011).

This model should motivate micro-society work that tests for effects of variation within groups in mechanistic (and autistic) and mentalistic (and positively schizotypal) cognition and skills in the origin and cultural evolution of technology. Such analyses should also provide novel insights into the nature of cognitive tradeoffs, their roles in psychiatric conditions, and their impacts on how humans have evolved to reach pinnacles of both technology and the arts.

Financial support. This work was supported by the Natural Sciences and Engineering Research Council of Canada (2019-04208).

Conflict of interest. None.

\section{Technical reasoning alone does not take humans this far}

\section{Maxime Derex ${ }^{a}\left([)\right.$ and Robert Boyd ${ }^{b}$}

\footnotetext{
anstitute for Advanced Study in Toulouse, 31080 Toulouse Cedex 06, France and ${ }^{b}$ School of Human Evolution and Social Change, Arizona State University, Tempe, AZ 85287-4101.

maxime.derex@iast.fr

robert.Boyd.1@asu.edu
}

doi:10.1017/S0140525X20000266, e162

\section{Abstract}

Although we see much utility in Osiurak and Reynaud's in-depth discussion on the role of what they term technical reasoning in cumulative culture, we argue that they neglect the time and energy costs that individuals would have to face to acquire skills in the absence of specific socio-cognitive abilities.

We commend Osiurak and Reynaud's (O\&R's) in-depth discussion of the role of what they call "technical reasoning" in cumulative culture (CC). There is no doubt that humans engage in complex forms of reasoning and a better appreciation of how this works is crucial for understanding what set humans apart from other animals. Contrary to what the authors suggest, Boyd et al. (2011) and Derex et al. (2019) never claimed that reasoning plays no role in CC. Rather they argued that the improvement of culturally evolving technology is not necessarily tied to individuals' level of understanding. Indeed, Derex et al.'s experiment shows that, over successive overlapping generations, participants produce increasingly efficient solutions despite exhibiting no improvement in causal understanding. This does not mean that causal reasoning cannot play a role in that process, but it shows that increases in efficiency are not necessarily accompanied (or even powered) by changes in individuals' understanding. These results illustrate the effects of the selective retention of beneficial modifications across generations and stress the roles of social learning and population-level processes in the emergence of adaptive cultural traits. That is not to say that the ability to reason has nothing to do with CC. Asking whether CC could occur in the absence of specific-reasoning abilities is an entirely different question. O\&R's proposal that CC necessarily depends on speciesspecific ability to technically reason about phenomena is both timely and welcome. Unfortunately, the evidence and arguments that the authors bring to bear in support of their hypothesis are weak.

First, Osiurak and Reynaud overestimate the ability of individuals to extract relevant information by observing artifacts alone. They base their claims on a few experimental studies that showed that even minimal social-learning mechanisms (such as reverse engineering) can result in cumulative improvements. According to them, "if signs of [CC] in reverse-engineering conditions are observed in humans, then this suggests that human non-social cognitive skills are sufficient for the emergence of [CC]." There are several problems with this argument. First, experimental investigations of CC rely on relatively simple tasks that participants can solve in a short period of time (Miton \& Charbonneau 2018). As a result, the amount of information that individuals can typically extract from the observation of such simple experimental artifacts is unrealistically high and whether technical reasoning skills allow individuals 
to infer substantial amounts of missing information about more ecologically valid artifacts remains to be demonstrated. Moreover, O\&R neglect the fact that experimental settings typically allow sustained and undisturbed observations maximizing the effectiveness of reverse engineering. Even if we assume that technical reasoning skills allow individuals to infer missing information, the usefulness of such capabilities in natural settings might highly limited in the absence of specific socio-cognitive abilities that give individuals appropriate access to cultural artifacts. Finally, we would like to point out that investigating information acquisition requires properly controlling for knowledge previously acquired by social learning. Indeed, O\&R's argument that information extraction is mediated by individuals' level of expertise suggests that they conflate information that was acquired during a specific learning event with information that was acquired prior to this learning event. To take O\&R's own example, showing that physics graduate students listening to Einstein retain more about the theory of relativity than individuals with no knowledge of physics might say less about what individuals actually learnt than what they previously knew.

Another problem with O\&R's argument is their claim that much can be learned by reverse engineering if learners can alternate between periods of social and individual learning and that socio-cognitive skills only boost CC. The fact that people can learn much through trial-and-error learning does not imply that socio-cognitive skills are unnecessary to CC. Even if we assume that individual learners could build a traditional Inuit kayak from a pile of driftwood and seal skins without learning from others (which is unlikely to say the least) that does not mean that they will do so in the absence of appropriate social support. More effective social-learning strategies do more than just change the rate of CC. Individuals constantly face intense tradeoffs and so must allocate their time and energy strategically. When learning costs are too high, individuals might not be able to afford to acquire complex skills by themselves. Experiments with children, for instance, show that they have difficulty innovating even simple tools even though they manufacture them easily after being exposed to social demonstrations (Beck et al. 2011). Moreover, being able to acquire a few skills through a combination of observational learning and individual leaning is not close to good enough. In the Arctic, kayaks are only useful if individuals can also develop warm clothes, harpoons and all other tools that their survival depends upon. Without appropriate socio-cognitive capabilities, the acquisition of these skills would require an investment in terms of time and energy that is way beyond what individuals can afford.

Finally, we would like to point out that experimental research has shown that toddlers are more likely to infer causal connections when sequences of events are the result of human actions rather than when they occur "naturally" without involving human interventions (Meltzoff et al. 2012). This means that reasoning skills are likely to at least partially result from socio-cognitive abilities that increase individuals' opportunities to witness valuable events. Furthermore, it suggests that hypotheses based on the role of reasoning skills should not be considered as alternative to those based on socio-cognitive skills. A more fertile approach may be to study how both types of abilities reinforce each other.

Financial support. This work was supported by the French Agence Nationale de la Recherche (under the Investissement d'Avenir programme ANR 17-EURE-0010).

Conflict of interest. None.

\section{A cognitive transition underlying both technological and social aspects of cumulative culture}

\author{
Liane Gabora ${ }^{\mathrm{a}}$ () and Cameron M. Smith ${ }^{\mathrm{b}}$
}

aDepartment of Psychology, University of British Columbia, Fipke Centre for
Innovative Research, Kelowna, BC V1V 1V7, Canada and ${ }^{b}$ Department of
Anthropology, Portland State University, Portland, OR 97207.
liane.gabora@ubc.ca
https://people.ok.ubc.ca/lgabora/
b5cs@pdx.edu
https://www.pdx.edu/anthropology/cameron-smith

doi:10.1017/S0140525X20000230, e163

\begin{abstract}
The argument that cumulative technological culture originates in technical-reasoning skills is not the only alternative to social accounts; another possibility is that accumulation of both technical-reasoning skills and enhanced social skills stemmed from the onset of a more basic cognitive ability such as recursive representational redescription. The paper confuses individual learning of pre-existing information with creative generation of new information.
\end{abstract}

The target paper's main thesis - that cumulative technological culture originates primarily not in social learning but in technicalreasoning skills - is consistent with results obtained with two computer models, both of which show that cumulative cultural evolution is possible in the absence of social learning (albeit at a slower pace) but not in the absence of mental operations akin to reasoning or creative cognition (Gabora 1995; 2008). Variants of the target paper's thesis have been proposed elsewhere (see Overmann \& Coolidge 2019). A competing theory not addressed in the target paper is that the cultural accumulation of both technical-reasoning skills and enhanced social skills stemmed from the emergence of some more basic cognitive ability. This theory enjoys support from psychology, anthropology, archeology, neuroscience, and genetics, and multiple versions of it (some not mutually inconsistent) have been proposed. Hauser et al. (2002) attribute cumulative culture to the capacity for recursion, as does Corballis (2011), who also emphasizes mental time travel: the capacity to think about events not occurring in the present. Others have argued that the general cognitive ability underlying cumulative culture was the onset of a self-triggered recall and rehearsal loop (Donald 1991), relational reinterpretation (Penn et al. 2008b), conceptual fluidity (Mithen 1996b), conceptual blending (Fauconnier \& Turner 2008), or something Chomsky (2008) called "merge." Our own two-step theory attributes cumulative culture to the onset of representational redescription followed by the capacity to shift between the convergent and divergent modes of thought, culminating in the emergence of an integrated internal model of the world (Gabora 2018; 2019; 2020; Gabora \& Smith 2018; 2019; Smith et al. 2018). Thus, although Osiurak and Reynaud (O\&R) position their technical-reasoning theory as the only alternative to social explanations for cumulative technological culture, they fail to consider theories that attribute it to the onset of a more general 
cognitive ability. Many of the arguments O\&R put forward in support of their theory are also compatible with, and supportive of, theories that attribute cumulative culture to a cognitive ability that paved the way for complex cognition in both the social and technological domains.

The authors highlight the distinction between sequential mechanical actions and combined mechanical actions, and between combined mechanical actions and genuine innovations (e.g., when they write "innovation in humans might primarily result from technical combinations rather than from novel inventions"). However, novelty does not depend on whether or not the elements are sequential (after all, notes of a song are sequential), nor is it something so simple as whether or not they are combined. The degree of novelty depends on the structure of the combination. The idea that nothing is truly new because innovation merely involves combining pre-existing elements was discredited decades ago with the discovery of emergent properties in concept (or word) combinations (Osherson \& Smith 1981), which have been shown to be not just present, but ubiquitous (Hampton 1987; Storms et al. 1998). Indeed, there is a field dedicated to studying, empirically (e.g., Scotney et al. 2020) and mathematically (e.g., Aerts \& Gabora 2005a; 2005b; Aerts \& Sozzo 2014; Bruza et al. 2012) the kinds of structure that emerge in combinations.

Throughout the paper, the authors espouse a sharp distinction between social and asocial learning (e.g., they write, "social $v s$. asocial learning"). However, consider the following scenarios for how a child learns to peel a banana: (1) by watching a sibling peel a banana, (2) by watching a monkey peel a banana, (3) by watching a cartoon monkey peel a banana, (4) by watching the petals of a smiley-faced cartoon tulip unfold, and (5) by watching the petals of a real tulip unfold. Where did we cross the line between social and asocial? One is forced to view social and asocial learning as ends on a continuum. The authors also assume that imitation and emulation are uniquely associated with social learning, but ask children in a theater or dance class to imitate leaves blowing in the wind and they know exactly what to do. (Indeed, efforts to emulate nature have given rise to much of what constitutes human culture.)

Related to this is a confusion in the paper between individual learning and creative cognition. Individual learning involves obtaining pre-existing information from the environment through asocial means (e.g., learning by oneself the distinctions between different kinds of butterflies), whereas creative cognition involves generating ideas, behavior, or artifacts that did not previously exist (Gabora \& Tseng 2017). This is important; supplying raw information is not the same as mental operations on this information. Individual learning and creative cognition contribute to cumulative culture in distinct, yet, complementary ways: the former (along with social learning) provides data about the world (e.g., discovery of electricity), and the latter brings something new into the world (e.g., invention of the flashlight). The distinction enables us to demarcate transition points in the evolution of complex cognition and in trajectories of actual technological lineages (Gabora \& Steel 2017; under review; Gabora et al. 2011; Veloz et al. 2012).

The authors curiously state that "working memory is not a cognitive mechanism that is used to generate content," but if so then where is content generated? Although incubation, intuition, and subconscious processing play a role in creative cognition (e.g., Bowers et al. 1995), the notion that generative capacities do not require working memory contradicts decades of research on the psychology of creativity. The authors also refer to "trial-and-error strategies that are not random but reasoned," but if the learning is "reasoned" then by definition it is not "trial and error."

It would be interesting to test the authors' hypothesis that "opaque" artifacts require more social learning for their transmission. Their notion of "opacity" is reminiscent of Bateson's (1979) notion of affordances, except that affordances arise dynamically in the interaction between observer and observed. We believe this distinction is important; those who contribute most to culture may be those who see possibilities that others miss.

Acknowledgments. This work was supported by a grant to Liane Gabora from grant 62R06523 from the Natural Sciences and Engineering Research Council of Canada.

Conflict of interest. None.

\section{Where does the elephant come from? The evolution of causal cognition is the key}

\author{
Peter Gärdenfors ${ }^{\mathrm{a}, \mathrm{b}, \mathrm{c}} \mathbb{\bullet}$, Anders Högberg ${ }^{\mathrm{b}, \mathrm{c}, \mathrm{d}}$ \\ and Marlize Lombard ${ }^{b, c}$
}

\begin{abstract}
a Department of Philosophy, LUX, Lund University, S-221 00 Lund, Sweden; ${ }^{b}$ Palaeo-Research Institute, University of Johannesburg, Johannesburg, South Africa; 'Stellenbosch Institute of Advanced Study, Stellenbosch 7600, South Africa and ${ }^{\mathrm{d}}$ Faculty of Arts and Humanities, Linnaeus University, 39182 Kalmar, Sweden.

Peter.Gardenfors@lucs.lu.se Anders.Hogberg@lnu.se mlombard@uj.ac.za

http://www.ht.lu.se/en/person/PeterGardenfors/

https://nu.se/en/staff/anders.hogberg/

https://www.uj.ac.za/contact/Pages/Marlize-Lombard.aspx
\end{abstract}

doi:10.1017/S0140525X20000059, e164

\begin{abstract}
Osiurak and Reynaud do not explain the evolutionary emergence and development of the elephant in the room, that is, technical cognition. We first argue that there is a tight correlation between the evolution of cumulative technological culture (CTC) and the evolution of reasoning about abstract forces. Second, intentional teaching plays a greater role in CTC evolution than acknowledged in the target article.
\end{abstract}

Boyd et al. (2011) created a divide between the "cognitive niche hypothesis" (e.g., Barrett et al. 2007; Pinker 2010) and the "cultural niche hypothesis." They define the cognitive niche in terms of evolutionary psychology as studied by Cosmides and Tooby (2001) and their followers. In our view, this delimitation amounts to a very restricted account of cognition. Thus, we agree with Osiurak and Reynaud (O\&R) that research concerning the so-called cultural niche has a strong tendency to ignore technical reasoning skills. In this respect, we also see the elephant in the room.

However, O\&R do not account for the evolutionary emergence and development of "the elephant." In section 2.2.1., paragraph 1, they write that "CTC emerges in humans because we possess the 
'dormant' technical potential" that other animals do not have, but they provide no explanation of how this dormant potential has arisen (with tongue in cheek we remind the authors of the "virtus dormitiva" in Molière's Le Malade imaginaire). Here, we provide a discussion on two topics that we see may help explain where the elephant comes from.

First, there is a tight correlation between the evolution of cumulative technological culture (CTC) and the evolution of causal cognition. We regret that O\&R (footnote 4) use the term "technical cognition" instead of "causal cognition," which is more common and more accurate for the topics they consider. There is co-evolution between technical engagement in the manufacturing and use of tools, and advanced forms of causal cognition in the human lineage. Human causal thinking has become detached from space and time, so that instead of just reacting to perceptual input, our minds can simulate actions and forces and their causal consequences. Unlike the situation for other primate species, an increasing emphasis on technical engagement made some hominins capable of reasoning about causal processes, for example, forces involved in tool use (Gärdenfors \& Lombard 2018). Tools extend the action field and action control of the users (Arbib et al. 2009). Because even the reactive fields of hand neurons are extended to the hand together with the tool, the use of tools is assimilated not only into the body schema, but also into neural circuits that effect cognition (Bruner \& Lozano Ruiz 2014; Maravita \& Iriki 2004). Thus, the way we think is profoundly rooted in and shaped by technical engagement. As an extension of these mechanisms, human causal cognition has evolved to become detached from what is present here and now, so that instead of simply reacting to perceptual input, our minds can simulate actions and forces and their causal consequences (Gärdenfors \& Lombard 2018; Penn \& Povinelli 2007). Such understanding of forces forms the core of what O\&R call technical-reasoning skills. Furthermore, causal thinking has become increasingly dependent on having a theory of mind (Lombard \& Gärdenfors 2017; Stuart-Fox 2014). In our opinion, an explanation of CTC should, at least in part, be based on the co-evolution between technical competence and causal cognition.

Second, intentional teaching plays a greater role in the evolution of CTC than acknowledged by O\&R, who claim that teaching might "not be the necessary condition for CTC" (sect. 3.3, para. 4) (in line with Derex et al. 2019). Several studies, however, emphasize the importance of intentional teaching - as opposed to learning by imitation only - in cultural transmission when learning complex, cognitively opaque skills such as the making of elaborate stone tools (d'Errico \& Banks 2015; Gärdenfors \& Högberg 2017). Indeed, intentional teaching is a fundamental human behavior, and from early childhood and onward humans cannot help teaching each other (Riede et al. 2018). Cross-cultural research underlines that it is indeed in economically and culturally highly valued domains where teaching is emphasized (Kline 2015), so that teaching is clearly beneficial for the transmission of technical knowledge.

Teaching is not only the presence of verbal interaction as in O\&R's Table 1, line 3. And there are two kinds of "observation" (Table 1, line 2): one where the learner is observing a model performing a task without directing itself to the learner. This is typically the case in chimpanzee nut-cracking (Lombard et al. 2019). The second type is where a learner is observing a teacher intentionally demonstrating or pantomiming how a tool is used or manufactured, which considerably improves the student's learning process (Gärdenfors \& Högberg 2017). Combined with verbal explanation of the hidden forces involved in performing the task, the learner can gain deeper understanding of the process and thereby be able to generalize and innovate. This process is also strengthened by the development of more advanced forms of a theory of mind in teachers and learners.

Many animal species have highly complex social structures, yet none has become obligatory stone tool users (Shea 2017; Zilhāo 2019), nor serial inventors and innovators of technologies as part of their survival mechanism the way humans have. For us, no separatist explanatory framework suffices for addressing "the elephant," or as O\&R put it "the increase in the efficiency and complexity of tools and techniques in human populations over generations." Instead, it is necessary to be increasingly aware of the fact that throughout our long evolutionary history there may have been different contextual emphases on a range of push and pull factors at play.

In short, we agree with O\&R that CTC is both a social and technological phenomenon. But it is our view that the technobehaviors that evolved along hominin lines cannot be maintained without intentional teaching, making them part of a cultural niche. At the same time, we argue that there is a co-evolution between technology and more and more advanced forms of causal cognition; and that theory of mind is a critical social cognition component of the causal cognition system so that in humans the cultural niche cannot be separated from the cognitive niche. From an evolutionary perspective, O\&R's assumption "that cumulative technological culture originates in non-social cognitive skills" is thus as limiting as the notion that causal understanding did not play a role in the development of culturally evolving technology (Derex et al. 2019).

Financial support. This research received no specific grant from any funding agency, commercial, or not-for-profit sectors.

Conflict of interest. None.

\section{A cognitive approach to cumulative technological culture is useful and necessary but only if it also applies to other species}

\section{Thibaud Gruber $\odot$}

Swiss Center for Affective Sciences, University of Geneva, 1202 Geneva, Switzerland.

thibaud.gruber@unige.ch

https://www.unige.ch/fapse/neuroemo/people/faculty/dr-thibaud-gruber

doi:10.1017/S0140525X2000014X, e165

\section{Abstract}

The debate on cumulative technological culture (CTC) is dominated by social-learning discussions, at the expense of other cognitive processes, leading to flawed circular arguments. I welcome the authors' approach to decouple CTC from social-learning processes without minimizing their impact. Yet, this model 
will only be informative to understand the evolution of CTC if tested in other cultural species.

Osiurak and Renaud defend the bold position that the invention of cumulative technological culture (CTC) does not depend so much on social processes than on intrinsic cognitive abilities, or technical-reasoning skills (TRS). This is clearly bold, because their position goes against the most dominant schools of thoughts, represented by Boyd and Richerson (Richerson \& Boyd 2005), Tomasello (e.g., Tomasello et al. 2005), their associates, and many others in the field, who explain the emergence of CTC through particular social-learning processes such as imitation and teaching (Tennie et al. 2009). I very much agree with the authors that too much weight has been given to sociallearning processes at the expense of other cognitive mechanisms, leading to circular arguments that only grant CTC to species displaying both imitation and teaching, and therefore excluding all nonhumans from CTC by definition (Gruber 2016). Yet, although I am supportive of decoupling CTC from its social components and generally agree that TRS must occupy a central place in the debate, this discussion should not be limited only to modern humans. Although the authors discuss intriguing findings in some nonhuman species at the beginning of section 2, they soon assert that (all) nonhumans have limited cognitive abilities connected to tool use, following Vaesen (2012), and evacuate rather than solve the problem of CTC in nonhumans. More problematically, the wording changes throughout the article, with CTC "at best, minimal in nonhumans" (sect. 2.2.1., para. 1) becoming completely "absent" (sect. 2.2.4., para. 1). There is, yet, a crucial difference between absence and acknowledging that some nonhuman behavioral variation is close to CTC, triggering discussion of limitations. In particular, such limitations probably applied to our early hominin ancestors, which are themselves seldomly discussed by the authors. This is problematic because these hominins likely built the foundations of our amazing and unparalleled CTC on the existing biological building blocks. In fact, the refocus on TRS could be a good way to approach behavioral continuity for CTC. My comment, thus, aims to extend the model of the authors to nonhumans, allowing sustaining an evolutionary continuity with our own ancestors.

Although impressive findings in animal tool cognition are discussed, they are only used to promote the idea that nonhumans are cognitively limited to understand their own tool use (sect. 2.1, para. 2). This position fails to acknowledge the large interand intra-specific variation between animal species, which is in fact suggestive of different cognitive skills, even within the same population, not so different than what the authors propose for modern humans. Failing to acknowledge differences in animal tool use from the most constrained and hardwired to the most "creative," as proposed by Call (2013), appears to me a biological mistake. In addition, starting from a null hypothesis where tool use might be cognitive to some extent in some species appears theoretically more sound in terms of evolutionary continuity, without hindering the fact that human tool use plays in a whole different category. I will exemplify this with one particular property of CTC: opacity, which the authors discuss in the context of theory of mind. Opacity, following Csibra and Gergely (2009; 2011), has been hailed as a marker of CTC in humans, as opposed to apparent "transparent" animal cultures. Yet, some animal cultural traits, such as termite-fishing in the Goualougou chimpanzees (Pan troglodytes, Sanz et al. 2009), combine complexity with limited information for the new learner to acquire visually (or "transparently"). Intriguingly, a recent report shows that this community displays more scaffolding of knowledge compared to a community with less complex tool use (Musgrave et al. 2020). In field experiments with wild chimpanzees in Uganda, stick-savvy Kanyawara chimpanzees did not evaluate sticks as opaquely as the stick-less Sonso chimpanzees (Gruber et al. 2009; 2011). Even more surprisingly, in the same experiment, male bearded capuchin monkeys (Sapajus libidinosus) do not evaluate sticks with the same opacity as females (Cardoso \& Ottoni 2016). Hence, there is evidence of variation in cognitive opacity between individuals across and within nonhuman species.

Given these results, it seems difficult to argue that modern humans are completely unique in having cognitive tool use, and subsequently, CTC. If this were true, then, an important question would be to determine when it occurred during our evolution. The classic view, heavily biased towards social learning, sees CTC mostly arising around the Acheulean period: It is argued that the complexity of bifaces makes it necessary for imitation to have been present to allow conserving the regularity in manufacturing these tools (Morgan et al. 2015). Yet, imitation is also used to justify the subsequent stasis that is observed for more than a million year (Tennie et al. 2017), being in fine responsible for both fostering and stalling technological progress. This illustrates that imitation or advanced social-learning skills remain mostly convenient one-fits-all explanations in the circular reasoning mentioned above. If, as proposed by the authors, one discards the idea that social mechanisms such as theory of mind or metacognition are essential to CTC and should rather be seen as "boosters"; and one agrees, as I argue here, that there is some kind of cognitive technological skills in nonhumans, there is much less of a theoretical conundrum regarding the appearance of sudden cognitive skills uniquely in the human lineage that allowed CTC to emerge. In addition, the problem of stasis mentioned above is no more about social learning, but rather about cognitive limitations. As illustrated by the authors, TRS also allows testing CTC with neurological data: If the endocasts of early possible human ancestors suggest that the parietal areas responsible for a cognitive appraisal of tools were already developed (Bruner 2018), we may conclude that CTC was already present in these species. The same reasoning could be applied to nonhumans that have controversially been granted CTC to assess whether homologous (Hopkins et al. 2017) or analogous brain structures (e.g., for corvid species) may indeed sustain CTC in nonhumans, offering an alternative but complementary evolutionary route to explain the unique rise in behavioral complexity in our lineage.

\section{Conflict of interest. None.}

Funding statement. This work was supported by the Swiss National Science Foundation grant CR13I1_162720/1. 


\section{Putting social cognitive mechanisms back into cumulative technological culture: Social interactions serve as a mechanism for children's early knowledge acquisition}

\section{Amanda S. Haber and Kathleen H. Corriveau (ㄷ)}

Boston University, Wheelock College of Education and Human Development, Boston, MA 02215.

haber317@bu.edu

kcorriv@bu.edu

doi:10.1017/S0140525X20000084, e166

\begin{abstract}
Osiurak and Reynaud offer a unified cognitive approach to cumulative technological culture, arguing that it begins with non-social cognitive skills that allow humans to learn and develop new technical information. Drawing on research focusing on how children acquire knowledge through interactions others, we argue that social learning is essential for humans to acquire technical information.
\end{abstract}

Drawing from research and theory from variety of disciplines, Osiurak and Reynaud (O\&R) offer a unified cognitive approach to cumulative technological culture, arguing that it begins with non-social cognitive skills that allow humans to learn and develop new technical information. We agree with the authors that technical reasoning can be considered a critical part of cumulative technological culture. However, although it is important to consider non-social cognitive skills, here, we argue that social learning is essential for humans to acquire technical information. Thus, an examination of developmental psychological research focusing on how children acquire knowledge through interactions with others is critical to understanding why social mechanisms are a central part of children's technical reasoning skills.

Children are curious learners who actively seek information from others, often caregivers. At an early age, infants have the capacity to engage in social referencing, using eye gaze (e.g., Brooks \& Meltzoff 2015), affective facial expressions and pointing to get an adult's attention (e.g., Butterworth 2003) and decide what to do in a situation. As children's linguistic abilities develop, children use questions and explanations from caregivers to acquire knowledge (e.g., Butler et al. 2020; Chouinard 2007; Frazier et al. 2009; Harris 2012; Harris et al. 2018; Kurkul \& Corriveau 2018; Ronfard et al. 2018). More specifically, children use testimony from adults to learn about a variety of topics including scientific (e.g., Callanan \& Oakes 1992; Tabors et al. 2001; Willard et al. 2019) and mathematical information (e.g., Hanner et al. 2019; Levine et al. 2010; Ramani et al. 2015). This is especially true when children are learning about scientific phenomena that are challenging to understand through first-hand observation alone such as the shape of the earth or the existence of germs. Similarly, acquiring an understanding of scientific processes such as electricity often require more than first hand-observation because the mechanisms are sometimes opaque or associated with a time delay (Corriveau et al. 2016; Harris \& Corriveau 2014; Harris \& Koenig 2006).

Moreover, children often rely on information from others to enhance their first-hand understanding of mechanisms underlying a causal process (Callanan \& Jipson 2001; Crowley \& Siegler 1999; Crowley et al. 2001a; 2001b; Haden 2010). To date, a substantial number of studies have found that through interactions with children, adult explanations serve as a source of information that significantly contribute to children's scientific understanding and learning (e.g., Jipson et al. 2016; Lombrozo et al. 2018; Vasilyeva \& Lombrozo 2018; Willard et al. 2019). Through parent-child conversations, children learn about causal mechanisms, demonstrate how to generate as well as test hypotheses and share important mechanistic information, all of which enhances 4- and 5-year-old children's ability to transfer knowledge to subsequent scientific activities (e.g., Kurkul et al. under review; Leech et al., in press). In their target article, $\mathrm{O} \& \mathrm{R}$ propose that cumulative technological culture emerges because of humans' non-social cognitive skills. Although technical reasoning, especially the ability to generalize what is learned from one situation to another one, is a fundamental part of the humans' capacity to develop new techniques or knowledge, we argue that these skills develop through interactions with others, suggesting that social mechanisms are integral to cumulative technological culture.

In sum, the unified cognitive approach to cumulative technological culture highlights the importance of technical reasoning skills in humans' ability to acquire and develop new content.

However, only focusing on the cognitive structures under this framework does not account for the fundamental role of social learning in the emergence of children's technical reasoning skills. We argue for a more integrated approach to cumulative technical culture, grounded in sociocultural theory (e.g., Vygotsky 1978). Such an approach should highlight the important cognitive skills children bring to bear to acquire technical information, yet also recognize that most learning situations occur in interactions with others. Thus, children's knowledge is strengthened through conversations with and observation of others.

Financial support. This work was supported by the National Science Foundation (grant number 1652224).

Conflict of interest. None.

\section{Human tool cognition relies on teleology}

\section{Mikołaj Hernik (1)}

UiT The Arctic University of Norway, N-9037 Tromsø, Norway. mikolaj.l.hernik@uit.no

doi:10.1017/S0140525X20000278, e167

\section{Abstract}

Osiurak and Reynaud's account of human tool cognition misses key element: human capacity for functional representations and teleological inferences. I argue that the teleofunctional approach 
accounts better for some features of human tool cognition and points to a viable candidate for the cognitive "difference-maker" behind human technological success.

Osiurak and Reynaud (O\&R) characterize human tool cognition that is, cognitive skills underpinning human tool use and cumulative technological culture - solely in terms of technical reasoning defined as physical-problem solving on the bases of mechanical knowledge. Here I argue that O\&R's account of human tool cognition is inadequate because of the omission of human propensity for functional representations.

Functional representations allow people to conceive of something (an object or an event) in relation to a future end-state, which they interpret both as effect of and explanatory factor for what they represent. This way actions and tools are interpreted as means toward ends, which are goals of actions. Such functional, or teleological, representations are present in the written record since antiquity (Aristotle), ubiquitous across cognitive domains in humans (Kelemen \& Rosset 2009; Rose \& Nichols 2019; Talanquer 2007) and evident from early on in human infancy (Gergely \& Csibra 2003; Hernik \& Csibra 2015; Liu et al. 2019).

Propensity for functional representations is not just a missing element in O\&R's presentation of human tool cognition. Teleofunctional account (Casler \& Kelemen 2007; Hernik \& Csibra 2009), which posits functional representations as the key element of human tool cognition, does overall a better job explaining some aspects of it, than O\&R's account focusing on technical reasoning alone does.

First, the teleological account allows for a more accurate characterization of everyday instances of tool-use. Technical-reasoning account presents them as instances of physical-problem solving, which results in selection of a tool and a way to operate it. Teleofunctional account allows for characterizing them simply as instances of goal-directed action involving tools. Some instances of goal-directed tool use certainly involve solving physical problems and decision processes described by O\&R (e.g., selecting the screwdriver with the tip small enough to fit the screw's aperture, or making do with a blade of a small knife instead). But remarkably, human tool use does not have to be always preceded by mental simulations and selection processes envisaged by $\mathrm{O} \& \mathrm{R}$. This is because humans form enduring functional representations of tools, where they conceive of any tool as being for doing something, that is, as a means to a certain type of goal. Humans indeed tend to use right tool for the job, but not because they go every time through the process of inferring the right means for the end. Rather, because they tend to use the tool for the job, that is, the tool that they already represent as the means to the goal that they intend to achieve. Propensity to rely on functional representations underlying tool-kinds is present also in cases of genuine physical-problem solving, as evidenced by so-called functional fixedness. Solving physical problem takes more time if it requires using an object to achieve a goal that differs from its already represented function (e.g., German \& Barrett 2005).

Second, the teleofunctional account allows for a fuller characterization of inferences that humans can draw in the domains of tools and actions. We do engage in reverse engineering in the sense discussed by $\mathrm{O} \& \mathrm{R}$, namely attempting to find out how does the tool do what it does. And we do engage in inferring means for the given ends, like in the tool-selection processes described by $\mathrm{O} \& \mathrm{R}$. In both cases $\mathrm{O} \& \mathrm{R}$ are right in pointing to the role of naïve mechanical principles in these processes. However, what O\&R's account does not recognize is that causal-mechanical principles alone do not provide sufficient constraints on such inferences. And for a simple reason: any state is compatible with a myriad of hypothetical causes and a myriad of hypothetical effects. Human cognition obviously resolves such inferences. However, it does so by relying on assumptions, which reach beyond the mere causal-mechanical principles, but are part and parcel of teleological representations, namely expectations of efficiency and optimality (Csibra \& Gergely 2007; Dennett 1990). Moreover, in addition to finding means for ends, humans engage also in the complementary inferential process and can find out the ends, when the means are given. A clear example of this in the action domain is goal anticipation (Southgate \& Csibra 2009). In the tool domain, this inverse teleological inference allows humans to infer what goals the object at hand facilitates achieving and thus to form hypothesis about its function (Csibra \& Gergely 2007).

Third, the teleofunctional account has better prospects of explaining development of tool knowledge, especially given the fundamental cognitive opacity (Gergely \& Csibra 2006) of tools and tool-use to the young observers. Rather than predicting that development of tool-knowledge has to track development of mechanical knowledge, teleological account predicts that it tracks capacity for teleological and functional representations. In other words, it predicts that even young human infants, despite poor capacity for tool-use and despite lack of detailed mechanical knowledge are nevertheless well equipped with the cognitive structures needed to learn about tool functions and tool kinds, because they are capable of representing means-end relations between actions and goals (Hernik \& Csibra 2015).

Recognition of the human capacity for functional representations and teleological inferences is missing from the O\&R's proposal, or perhaps it is only tacit in it. This is a striking omission for two reasons. First, these cognitive capacities are arguably prerequisites of the human technological success. They enable constrained inferences of means from ends and ends from means. They can support learning from observed instances of tool-use by extracting the key means-end-relations with only cursory understanding of the underlying causal relations. They enable ascribing lasting value to unused tools by representing them as means for future goals. Second, even though non-human primates may engage in teleological inferences (Rochat et al. 2008), there is only limited evidence that they may form end-from-means inferences, lasting functional representations of tools (Mulcahy \& Call 2006) and function-based tool categories (Gruber et al. 2019). Thus, the human teleofunctional representations are at least as good a candidate as the technical reasoning skills are for the cognitive "difference-maker" behind the human cumulative technological culture that O\&R are after (Hernik \& Csibra 2009).

Financial support. This research received no specific grant from any funding agency, commercial, or not-for-profit sectors.

Conflict of interest. None. 


\section{How will we find the elephant in the room?}

\section{Wybo Houkes and Krist Vaesen 1}

Philosophy \& Ethics, Eindhoven University of Technology, Eindhoven, The Netherlands.

w.n.houkes@tue.nl k.vaesen@tue.nl

doi:10.1017/S0140525X20000229, e168

\section{Abstract}

We argue that Osirak's and Reynaud's technological-reasoning hypothesis raises conceptual and methodological challenges. Interrelations between technical potential and expertise leave it unclear exactly what the technical-reasoning hypothesis encompasses. We submit that it is compatible with a range of hypotheses that are difficult to differentiate empirically.

Osiurak and Reynaud (O\&R) draw attention to "the elephant in the room": an overlooked set of cognitive mechanisms that could explain cumulative technological culture (CTC) in human populations. In particular, their technological-reasoning hypothesis states that all humans possess unique, non-social cognitive skills, dubbed their technical potential, "enabling them to acquire and generate a great - perhaps infinite - amount of technical information," dubbed their technical expertise (sect. 1, para. 3).

There is no doubt that technical-reasoning skills play a role in CTC. Knowing how to design or use artifacts crucially involves understanding how sequences of actions affect physical mechanisms: humans deliberately press buttons, stir mixtures, or flip switches in order to trigger, affect, or terminate processes in our devices. Such action sequences, codified in recipes or user manuals, resist specification in terms of separate motor actions; rather, the technical information or expertise involved in use and design can be analyzed in terms of interdependent sets of actions or plans (Houkes \& Vermaas 2010). There is, moreover, no doubt that one's repository of technical information may increase over time, for instance through the application of technical-reasoning skills to episodes of successful use: one may come to recognize which actions reliably lead to which results by means of which artifactual physical mechanisms. Conversely, expertise may improve technical-reasoning skills. Through (reflection on) repeated use, one may enhance skills for specific technologies (improved causal reasoning), or may discern similarities between technologies (improved analogical reasoning).

Accepting these interrelations between technical potential and expertise leaves it unclear, however, exactly what the technicalreasoning hypothesis encompasses. Conceptually, it is compatible with a range of hypotheses that, methodologically, are difficult to differentiate empirically.

At one extreme, one may hypothesize that technical reasoning (i.e., human technical potential) may be necessary for CTC, but that this potential remains elementary in virtually all of us, despite growing technical expertise. Both simple and sophisticated recipes, for instance, are comprised of relatively elementary mechanical actions such as "stirring" and "cutting," which are not too far above the level of motor actions. At the other extreme, one may hypothesize that technical potential grows in tandem with technical expertise: expert pianists may discern action-result patterns that are beyond the capacities of any novice, both in terms of describing higher-order mechanical actions and in terms of perceiving produced effects.

The range of hypotheses is increased further by considering the role of social (cognitive) mechanisms. Technical expertise grows in part because of mentoring and social interaction, as we guess O\&R would agree with. Still, it is conceivable that we may acquire great expertise in, say, cycling exclusively through individual use and technical reasoning skills - where such reasoning skills may, in turn, be relatively stable over time or, rather, vastly improve. Conversely, one may hypothesize that social interaction is necessary for increased expertise, or for acquiring a certain level of expertise. Furthermore, one may differentiate these hypotheses according to different types of technologies: the interaction between technical potential, expertise, and social interaction may be different for, say, cooking than it is for, say, civil engineering.

In light of this, we submit that the main contribution that O\&R have made is not so much substantiating one particular hypothesis (viz., their technological-reasoning hypothesis) as revealing a large range of hypotheses regarding the mechanisms that underlie CTC.

By consequence, much care is required, conceptually, to distinguish between various possible hypotheses. Notions such as "potential" and, in particular, "expertise" easily give rise to confusion or to trading on ambiguities: "expertise" may refer to, among other things, a body of more or less codified knowledge, or to skillful use (thus including technical-reasoning skills). Moreover, each hypothesis within the range of aforementioned hypotheses requires careful formulation. It will not do, for instance, to argue that CTC may result from technical-reasoning skills alone by simply providing evidence that the latter necessarily complement social cognitive mechanisms.

Methodologically, enlarging the range of hypotheses has more problematic consequences. Once appropriately formulated and disambiguated, many more hypotheses turn out to be compatible with the available evidence than most parties in this debate appear to realize. We may reasonably claim that technological-reasoning skills play a role in CTC, but this leaves open many issues: whether technological-reasoning skills are more than a necessary complement to social-cognitive mechanisms; how these skills in humans can be characterized independently of growing expertise and social interaction; and to what extent these skills are global or specific to technological domains. Moreover, these issues are not just unresolved at present - it is difficult which evidence could speak in favor or against, say, claims that technological-reasoning skills alone (i.e., without social interaction) suffice for attaining large, "perhaps infinite" levels of expertise.

To be sure, that the available or possible evidence may underdetermine explanatory hypothesis concerning CTC is a problem for anyone interested in this phenomenon and not only for the authors of the target article. Yet to return in closing to their leading metaphor: we may concede their point that there is an elephant in the room, but submit that it may be small, heavily domesticated, or simply impossible to locate because of all the people.

Acknowledgment. Research by Krist Vaesen was supported by the Netherlands Organisation for Scientific Research (NWO, Vidi-grant 276-20-021).

Conflict of interest. None. 


\section{Tools as "petrified memes": A duality}

\section{Carsten Korth (1)}

Heinrich Heine University Düsseldorf, Moorenstrasse 5, 40219 Düsseldorf, Germany.

ckorth@hhu.de

https://www.uniklinik-duesseldorf.de/en/institute-of-neuropathology/research/ ag-korth

\section{doi:10.1017/S0140525X20000205, e169}

\section{Abstract}

Tools are generated by defined steps, fulfill distinct uses, and elicit affordances or mental representations. When the latter are recombined, they are perceived as "technical reasoning," resulting in novel tools when executed. They can be exchanged, varied, and selected between individuals in a cumulative social process. Tools are materialized, "petrified" memes forming a duality within the framework of active externalism.
\end{abstract}

In their target article, Osiurak and Reynaud (O\&R) emphasize that "technical reasoning" critically contributes - as an individual ability - to cumulative technological culture (CTC). I comment on several of their statements in the context of a novel, coherent theoretical perspective.

I here conceptualize tools to be in a duality with their corresponding mental representations, as kinds of mirror images. Such a view is supported by active externalism (Clark \& Chalmers 1998; Menary 2010) stating that (brain-) external objects are directly coupled to cognitive abilities that otherwise could not happen. For example, mathematics is a cognitive activity that beyond the limited capacity of mental arithmetics - cannot be performed without tools, that is, external objects.

The term "tools" here is used in its widest sense which includes artifacts. Tools are produced in a defined sequence of observable acts and serve one or several uses. Tools elicit two kinds of affordances: first, those for their use, and second, mental representations on how they were produced; for simple objects, these mental representations may be accurate to detail ("ladder" and "hammer"), but for complex tools these may be diffuse ("computer"). These affordances can be described as mental representations or instructions corresponding to externalized, clearly defined action sequences of observables (see Fig. 1).

Mental representations can be transmitted between individuals and executed whereby the tool is reproduced. Or they can be varied, including by reshuffling, for example, decomposition and reassembly to novel mental representation sequences. A reshuffled mental representation, corresponding to "technical reasoning," when executed, will generate a novel, innovative tool. Tools are therefore materialized, or "petrified" memes because the previously modifiable, corresponding mental representations have been fixed and are "archeological" evidence of that prior, observable and discrete sequence.

I propose that such an exclusive and restricted definition of memes in the context of tools and their corresponding mental representations can be a useful, unifying, and fertile concept for bridging macroscale and microscale phenomena, that is, CTC and individual psychology, respectively. Such a definition as a tool-meme duality contrasts to previous fuzzy definitions that attempted to be all-comprising but were ultimately unproductive.

The meme shuttles between a representational stage (coded in the brain by unknown mechanisms) and materialized stage molded in the tool (see Fig. 1). The material stage increases meme fidelity and thereby potentiates its replicating power. Tool evolutionary history can, in principle, be tracked in a pedigree-like manner.

The question is not whether a meme is necessary (Henrich et al. 2008) but whether it can be defined in a useful way providing an instrument for novel transdisciplinary insights. Universal Darwinism (Dawkins 1983) posits that if an algorithm is active that includes variation, replication, and selection, the emergence of replicators is inevitable.

The definition that $\mathrm{O} \& \mathrm{R}$ use for structure and content corresponds to the classical definition of vehicle and replicator (Dawkins 1976; 1982), respectively. The more prior tool-related mental representations (replicators) are present in an individual vehicle the less opaque and the more transparent is a tool perceived.

This is not in contradiction to the possibility that individual "hardware" (e.g., brain wiring) differences may exist. The individual ability to process mental representations will be a sum of both software (relevant memes uploaded) and hardware (individual processing, recombination, or affordance abilities).

Within the dual inheritance theory, scientific evidence and a clear framework have been established to explain mutual influencing between cultural (software/content) and biological evolution (hardware/structure) (Boyd \& Richerson 1985; Henrich 2016).

Inborn behavioral patterns are more consistent and faster available, whereas acquired behaviors are flexible but prone to eradication. Culture-biology coevolution likely has used both modes and combined their advantages. Useful, acquired behaviors have been fixed to make them less labile ("Baldwin effect") and inborn behaviors have been replaced by more flexible modes. The speed of such a culture-biological coevolution is accelerated enormously by assortative mating (Creanza et al. 2017) and is demonstrated by domestication (Hare et al. 2005), or selfdomestication (Hare 2017; Henrich 2016).

Ultimately, at any given moment, there exists a mosaic of hardware- and software-based processing for the brain with all possible graduations - all simultaneously present but each with different phylogenetic and ontogenetic histories.

The existence of specialized brain areas participating in recalling particular kinds of memes (i.e., for technical reasoning area $\mathrm{PF}$ ) is conceivable: motor tasks and corresponding mental representations may evolve from simple sequential arrangements to those involving objects, and later tools. During this gradual, phylogenetic evolutionary trajectory, specific brain areas are sequentially involved and occupied.

O\&R presume that technical reasoning is able to extract "essences." "Essences" could be elegantly defined as the explicit perception of overlapping features of a large number of toolrelated mental representations.

O\&R oppose social versus asocial learning and explicitly name technical reasoning as an example for an asocial skill. This distinction ultimately depends on the definition of social learning (Heyes 2012). Although technical reasoning does not require the presence of other individuals, clearly, all technical mental representations themselves have been "uploaded" at some point in time by social learning (including pedagogy) which is domaingeneral (Heyes 2012). Once uploaded, of course, mental representation can run off in an asocial mode. 
Figure 1 (Korth). Scheme on the tool-mental representation duality showing the flow between the individual's mental representations and tools in replication cycles. Mutations happen in the internal, private sphere, replication and selection happens in the external, public sphere.

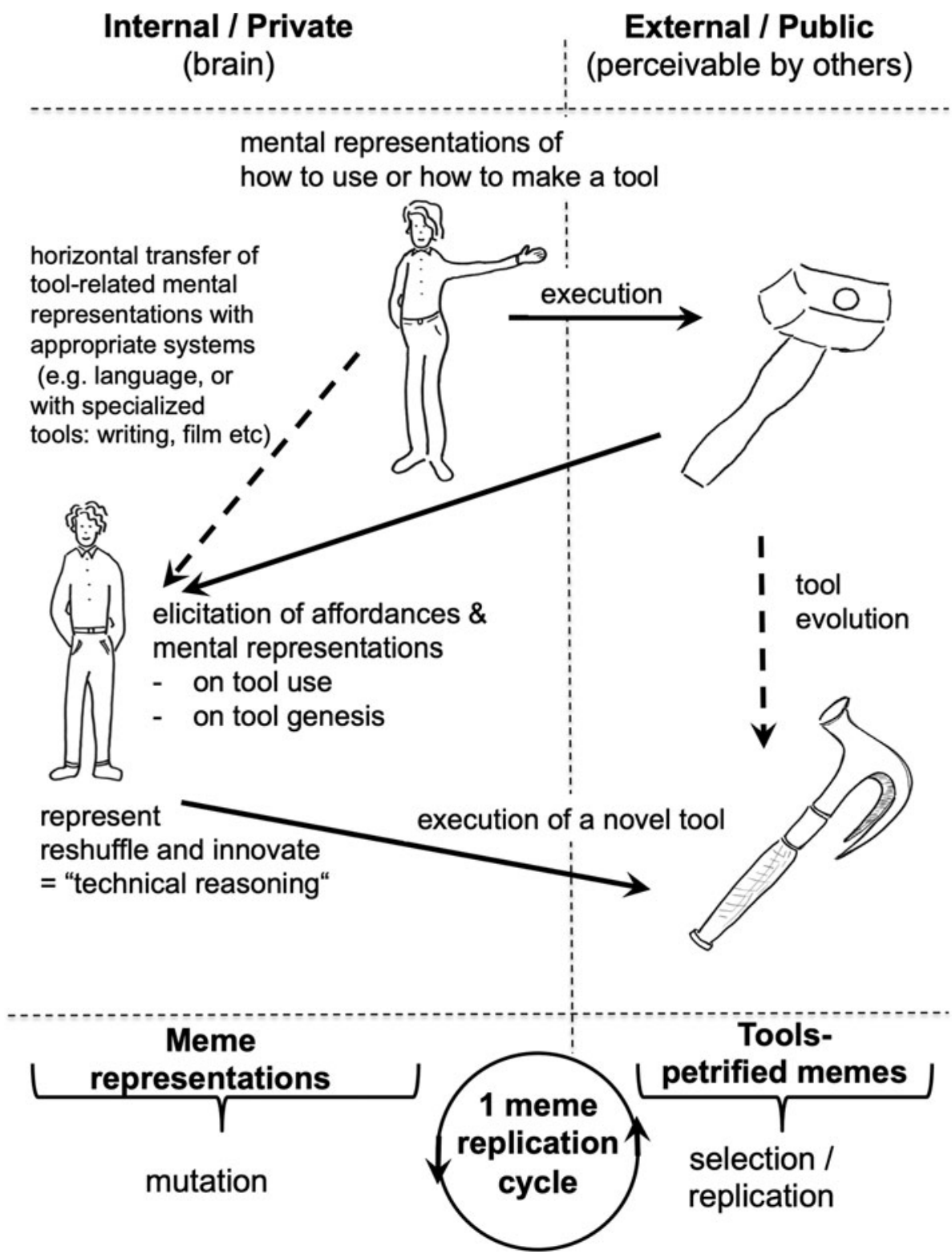

For a comprehensive theory of technical reasoning and innovation, the importance of group size and social communication for acquiring technical skills can also integrated by the toolmental representation duality: experimental evidence shows that social transmission skills win over individual innovation skills at the group level, as long as there are a few innovators present because technical reasoning is more easily copied than reinvented (Henrich 2009; 2016, p. 213; Muthukrishna et al. 2014).

In conclusion, technical reasoning within CTC cannot be seen outside an ontogenetic and phylogenetic context involving dual inheritance, and a restricted definition of memes as a tool-mental representation duality may be a fertile ground to elegantly bridge these areas.

Financial support. This research received no specific grant from any funding agency, commercial or not-for-profit sectors.

Conflict of interest. None.

\section{Missing in action: Tool use is action based}

Jeffrey J. Lockman ${ }^{\circledR}$, Catherine S. Tamis-LeMondab ${ }^{\text {(1) }}$ and Karen E. Adolph ${ }^{\mathrm{C}}$

\footnotetext{
${ }^{\mathrm{a}}$ Department of Psychology, Tulane University, New Orleans, LA 70115;

${ }^{b}$ Department of Applied Psychology, New York University, New York, NY 10003 and ${ }^{\mathrm{C}}$ Department of Psychology, New York University, New York, NY 10003.

lockman@tulane.edu catherine.tamis-lemonda@nyu.edu karen.adolph@nyu.edu

https://childdevelopment.tulane.edu/ https://wp.nyu.edu/

catherinetamislemonda/ http://www.psych.nyu.edu/adolph/
}

doi:10.1017/S0140525X20000138, e170 


\section{Abstract}

In this commentary on Osiurak and Reynaud's target article, we argue that action is largely missing in their account of the ascendance of human technological culture. We propose that an action-based developmental account can help to bridge the cognitive-sociocultural divide in explanations of the discovery, production, and cultural transmission of human tool use.

Tool use is inherently about action. But action is largely missing from Osiurak and Reynaud's (O\&R's) account of technical reasoning skills and the emergence of cumulative technological culture. We argue that a meaningful account of the rapid escalation of human tool use must include the action systems that are fundamental to the discovery, production, and cultural transmission of tools. Furthermore, we suggest that by incorporating action into explanations for the ascendance of human tool use, we can integrate technical reasoning and sociocultural approaches and thereby bridge this cognitive-sociocultural divide.

Our proposals are rooted in perception-action theory (Gibson $1979 ; 1982)$ and the protracted period of skill acquisition that characterizes motor development in human infants and children (Adolph \& Robinson 2015). From a perception-action perspective, objects, including tools, change the properties of the body's effectors (typically the hands and arms in humans). An object in hand can enrich or create new opportunities "affordances" - for action (Adolph \& Robinson 2015; Lockman 2000); for example, empty hands do not easily afford hammering, but hard graspable objects do. Equally important, humans display exquisite, efficient exploratory procedures (Lederman \& Klatzky 1987) to perceive which actions with which objects lead to which outcomes. Exploration enables people to discover an object's potential as a tool or the means to implement an existing tool. Information that specifies an object's potential to serve as a tool is often readily available in the environment and does not depend on the technical reasoning skills proposed by O\&R. Even 8- to 10-month-old infants combine objects and environmental surfaces selectively based on the physical properties of each - a critical element for successful tool use (Fontenelle et al. 2007). Similarly, other tool-using species, such as Capuchin monkeys (Visalberghi et al. 2009a) and New Caledonian crows (Chappell \& Kacelnik 2002) explore and select tools geared to the demands of a given problem, thereby optimizing the outcome.

A developmental lens sheds further light on the process of tool use. Although information about an object's potential as a tool may be discoverable through exploratory procedures, successful tool use often requires extended periods of practice, especially early in development. In tool-using species such as chimpanzees, Capuchin monkeys, and New Caledonian crows, juveniles spend long periods of developmental time practicing action patterns that are subsequently incorporated into tool use (Inoue-Nakamura \& Matsuzawa 1997; Kenward et al. 2011; Resende et al. 2008). Similarly, human children spend several years hammering objects against other objects before they display the distinctively human pattern of controlled wrist flexion required for hammering accuracy (Kahrs et al. 2014). In short, the road to tool use entails much more than technical reasoning skills - motor skills must be refined; grips must be strengthened; and muscles and joints must be coordinated.
Perception-action exploration and motor skill learning are also fundamental to the discovery and implementation of actions when the affordance is not immediately apparent - that a lid twists, and it's necessary to turn to the left, not to the right. Such "hidden" affordances are common in artifacts - the required action is non-obvious but necessary to use the object as intended by the designer (Gaver 1991; Rachwani et al. 2020). Although technical reasoning may help to narrow the candidate affordances considered for further exploration, perceptual-motor activity is critical to discover and implement the designed actions of objects. A developmental lens is again instructive. When 11- to 37-month-old children are given containers that require a twist-off or pull-off action to open, the youngest children display non-designed actions such as banging or shaking; slightly older children attempt the designed action but frequently fail to open; and only the oldest children successfully implement the designed action (Rachwani et al. 2020). Moreover, the lag between displaying a designed action and successful implementation extends for months or years. And so, technical reasoning alone does not ensure successful implementation. Children may know which action to perform, but knowledge does not guarantee success.

An action perspective can also enrich accounts of the mechanisms that promote sociocultural transmission of tool use. Our culture creates "user-friendly" tools and devices that advantage the morphology of the human hand and manual function. Thus, the designed affordances of human tools can shed new light on the ratcheting and social transmission mechanisms that underlie the growth of human technological culture across generations. Because the implementation requirements of many artifacts leverage the highly precise dynamics of fingers, hands, and arms (e.g., oscillatory dynamics of the arm are recruited to hammer; pressing actions of the fingers are engaged to type on a keyboard; pushing, pulling, and rotational movements of the hand/arm are enlisted to fit objects, plug in power cords, and so on), and because humans continually seek to modify artifacts to improve their fit with the constraints of the human body, the transmission of technology accelerates and ratchets up. Here, development again comes into play. The technological culture adapts tools to meet the limited skills of infants and children. Many cultures create smaller-scale replicas of tools and other artifacts for children (Lancy 2017). Similarly, changes in children's hand size and dexterity are the impetus to create developmentally appropriate artifacts to facilitate (e.g., easy-to-manipulate toys) or hinder (e.g., child-resistant packaging) young children's use of artifacts. And artifacts are modified to facilitate implementation in elderly people with diminished manual capacity. Collectively, these action-driven practices promote the transmission of human technological culture from one generation to the next.

To conclude, we agree with O\&R's claim that the mechanisms that enabled transmission of human technological culture across generations are only incompletely understood. We argue that action must be the centerpiece of any account of human technological culture and observations of developmental change are instructive for understanding cultural change. An action-centered, developmental account can begin to bridge the cognitive-sociocultural divide in explanations of the discovery, production, and cultural transmission of human tool use.

Financial support. This work was supported by the National Institute of Child Health and Human Development (grant number R01-HD086034).

Conflict of interest. None. 


\section{A theory limited in scope and evidence}

\section{Elena Miua ${ }^{a}$, Robert Boyda ${ }^{a}$ Peter J. Richerson ${ }^{b}$}

\section{and Thomas J. H. Morgan ${ }^{\mathrm{a}}$ ()}

${ }^{a}$ School of Human Evolution and Social Change and Institute of Human Origins, Arizona State University, Tempe, AZ 85287 and ${ }^{\mathrm{b}}$ Department of Environmental Science and Policy, University of California Davis, CA 95616.

emiu1@asu.edu

Robert.Boyd.1@asu.edu

pjricherson@ucdavis.edu

thomas.j.h.morgan@asu.edu

\section{doi:10.1017/S0140525X20000035, e171}

\section{Abstract}

What promised to be a refreshing addition to cumulative cultural evolution, by moving the focus from cultural transmission to technological innovation, falls flat through a lack of thoroughness, explanatory power, and data. A comprehensive theory of cumulative cultural change must carefully integrate all existing evidence in a cohesive multi-level account. We argue that the manuscript fails to do so convincingly.

Osiurak and Reynaud's (O\&R's) theory of technical reasoning does not advance our understanding of cumulative cultural evolution. There are four important deficiencies. First, it does not make a novel contribution. We agree that the study of cumulative cultural evolution would benefit from a better understanding of technological reasoning. However, this idea is not novel. A number of authors have suggested that theories of cultural evolution need to better integrate reasoning processes (Claidière et al. 2014a; Heyes 2012). It is not enough to present another a verbal argument for the importance of technological reasoning. Real progress requires an account of how such reasoning works. What existing cultural evolutionary work does well is provide a theory that explains how individual-level decisions scale up to population-level dynamics (e.g., Mesoudi 2016; Morgan 2016), including experimental studies that investigate individual cognition, phylogenetic approaches that characterize macro-evolution, and theoretical models that link the two. A theory of technological reasoning should link how individuals reason about a task to the innovations they produce, and finally to cultural dynamics. The manuscript is lacking in this regard.

Second, the authors make numerous empirically testable claims without sufficient evidence to move relevant debates forward. For instance, they criticize the cultural niche theory for downplaying the importance of technological reasoning. However, this is a disagreement that can be solved empirically: to what extent does data suggest that cumulative cultural change is constrained and/or directed by technological reasoning? The authors offer frustratingly little hard data for the relevance of technical reasoning. A compelling theory would go beyond stating that technical reasoning is important and, instead, offer evidence that quantifies this importance, shows how technical reasoning shapes cultural change, and makes clear the limitations of cumulative cultural change in its absence. At present, the authors cite only a single (self-authored) study, which does little to convince the reader of the bold statements made in the manuscript.

Third, we question the assertion that individuals can always produce any single solution produced by cultural evolution. To quote the authors, "all of us are smart enough to acquire each piece of information - as well as to produce any kind of innovation - necessary to survive in any single habitat." This claim is empirically dubious. For example, the Polar Inuit lost a number of important skills during an epidemic in the early nineteenth century, including kayaks, bows, and important aspects of snow house construction. For 40 years or so these people lived without these important tools, and none of the several hundred people in the group were able to reinvent them. When a group of Baffin Island Inuit visited them around 1860, they immediately reincorporated them into their technological repertoire (Boyd et al. 2011). There are many other examples (Earl \& McCleary 1994). This claim also neglects the fact that cumulative cultural evolution changes the nature of the problems that must be solved. Cumulative cultural evolution not only involves building upon and improving technology from previous generations, it also opens up new niches with new problems. The invention of the wheel, writing, the internet, all introduce new possibilities and new problems to solve. Perhaps more broadly, life in larger groups generated both a need for norms and institutions that maintain cooperation, as well as more potential for innovation. Would a single human, of infinite lifespan, with infinite time, be able to produce the entirety of the current human repertoire? The authors seem to suggest that this would indeed be the case, but competing accounts suggest that it is not individual cognition, but social complexity and inter-connectedness that are responsible for our technological success (Muthukrishna \& Henrich 2016). Whether the authors' claim is true or not remains a question unanswered here.

Fourth, the authors emphasize technological reasoning as key to technological change in cumulative cultural evolution, but cumulative cultural evolution involves a large range of domains other than technology: cooking, language, norms, art, knowledge, abstract or not, all change as a result of cultural transmission and become increasingly complicated. A great deal of knowledge essential for survival, like knowledge about hunting techniques and plant detoxification, does not involve technical reasoning of the kind described by O\&R. The domain of language, as well, is very different. Although technological products are shaped by functional pressures (a good bow shoots far and accurately), languages serve communication with symbols defined by convention. The exact form of words is irrelevant, as long as everybody agrees on the same form, and languages are shaped by expressivity and learnability instead (Kirby et al. 2008). Art is, perhaps, at the opposite end of the spectrum from technology. It is free to change according to our esthetic preferences, without any objective benchmark to measure against, and intuitive physics is no help in identifying successful designs. Nonetheless, complexity increases as a result of cultural transmission processes, without any need for technical reasoning abilities. If technological reasoning is the key to human uniqueness, it must explain this plethora of culturally governed processes that fall outside of the technological domain.

Finally, the authors' focus on technological reasoning as "necessary and sufficient" conditions for cumulative cultural change is misguided. Existing theory clearly shows that cumulative cultural change can occur through a number of mechanisms, ranging from a guidedvariation/technical-reasoning model to a selective-transmission-of- 
random-variation model (Mesoudi et al. 2016). There is no need for "magic bullet" theories, especially when the current state-of-the-art theories suggest that complex cycles of co-evolution of cognition, life-history, social structure and culture are responsible for our species' success (Boyd 2017; Henrich 2016; Laland 2017a).

Financial support. This research received no specific grant from any funding agency, commercial, or not-for-profit sectors.

Conflict of interest. None.

\section{Shared intentionality shapes humans' technical know-how}

\section{Henrike Molla ${ }^{\oplus}$, Ryan Nichols ${ }^{b}$ and Ellyn Pueschela}

\footnotetext{
${ }^{a}$ Department of Psychology, University of Southern California, Los Angeles, CA 90089 and ${ }^{\mathrm{b}}$ Department of Philosophy, California State University Fullerton, Fullerton, CA 92831.

hmoll@usc.edu
}

doi:10.1017/S0140525X20000163, e172

\begin{abstract}
Osiurak and Reynaud argue that cumulative technological culture is made possible by a "non-social cognitive structure" (sect. 1, para. 1) and they offer an account that aims "to escape from the social dimension" (sect. 1, para. 2) of human cognition. We challenge their position by arguing that human technical rationality is unintelligible outside of our species' uniquely social form of life, which is defined by shared intentionality (Kern \& Moll 2017, Philosophical Psychology 30(3):319-37; Tomasello 2019a, Becoming human: A theory of ontogeny. Cambridge, MA: Belknap Press).
\end{abstract}

Osiurak and Reynaud (O\&R) argue that cumulative technological culture (CTC) is made possible by a "non-social cognitive structure" (sect. 1, para. 1) enabling humans to acquire technical knowledge and skills. They maintain that CTC "is necessarily based on our extensive individual cognitive ability [our emphasis] to acquire and improve techniques" (sect. 1, para. 3), and they offer an account that aims "to escape from the social dimension" (sect. 1, para. 2) of human cognition. The implication seems to be that social cognition is not necessary for humans to develop fundamental technical skills.

We believe that an escape from human sociality cannot succeed because the social nature of human intelligence permeates all aspects of human cognition and cumulative culture. Human technical and instrumental rationality are unintelligible outside of our species' uniquely social form of life, which is defined by shared intentionality (Kern \& Moll 2017; Tomasello 2019a). We will deliver two points to make our argument. The first point casts doubt on O\&R's thesis that humans' "technical potential" is fundamentally a feature of individual intentionality and instead suggests that humans' technical know-how is rooted in acts of shared intentionality. The second point is methodological. We will argue that the micro-society experiments $\mathrm{O} \& \mathrm{R}$ cite in support of their position do not constitute compelling evidence in favor of the asocial origins of technical knowledge and understanding.
The first point is informed by cognitive developmental psychology. Studies suggest that children do not develop their technical know-how by trial and error or solipsistic hypothesis testing. Instead, their instrumental rationality is shaped in acts of shared agency with competent adults who show them how to use and craft tools and address instrumental problems (Call \& Tomasello 1995; Moll 2018). Let us give two examples. It has been established that young children have difficulties to consider water as a tool. When asked - in the absence of any solid tools but in the presence of water - to extract a small object from the bottom of a narrow and deep tube, preschoolers do not come to think of the possibility of pouring water into the tube to make the object float atop (Hanus et al. 2011; Moll 2018). However, when the instrumental usefulness of water is pedagogically introduced to them, most children spontaneously identify the solution and extract the buoyant object by releasing water into the tube (Moll 2018). Another example is provided by young children's tendency to "over-imitate," even when the mechanical structure of a device is entirely transparent (Lyons et al. 2007; McGuigan et al. 2007). When shown irrelevant in addition to relevant action steps in the course of a transparent apparatus' manipulation, most children faithfully imitate the entire procedure, including the irrelevant steps. If humans' technical abilities can be explained by a "non-social cognitive structure," as O\&R claim, then it would seem that children should be immune to over-imitation and selectively reproduce only the relevant steps. The fact that most children faithfully stick to the adult's demonstration testifies to the significance of social trust in epistemic and technical matters. This trust is rational because in a social world replete with arbitrary conventions, symbolic communication, rituals, and common occurrences of "causation at a distance," it is often too difficult to determine for individual young learners why, or how, something is causally effective.

The second point concerns O\&R's claim that micro-society studies prove that individuals can "reverse-engineer" artifacts without any social assistance. Granted, adult individuals can, under certain conditions, deduce the production process of certain artifacts simply by inspecting the end product. But it is doubtful that these individuals would be able to reverse-engineer anything without an extended social learning history in which they were introduced to the use and manufacturing of various tools and other artifacts. Imagine someone with a history like that ascribed to Kaspar Hauser. It is unlikely that this person could individually make out the function of, say, a can opener. Humans' social learning experiences shape their grip on how artifacts are constructed. Because micro-society experiments cannot control for the participants' social biographies, their validity as measures of what can be attributed to individual versus shared intentionality is dubitable. In fact, it can be difficult even for adults with normal socialization histories to individually discern an unfamiliar tool's purpose. In a small study $(N=21)$ we conducted with adults (eight males) between 20 and 68 years ( $M=30$ years), participants were given a cherry/olive pitter and asked what the device is. A single participant gave the right answer; most answers (incl. the modal response "hot glue gun"), were far off. It thus seems that humans' technical understanding shows clear limits without a meaning-providing cultural context, be it in the form of others' demonstrated use of an object or tool shops with labeled object categories, and so on.

With these points of critique, we hope to have shown that human technological culture and its propagation cannot occur without epistemic and technical transactions involving other agents who master the "technai" that render cultural products 
accessible for use and reproduction. Human sociality is irredeemably written into humans' technical capacity.

Financial support. The Albert and Elaine Borchard Foundation supported this work with a grant awarded to HM and RN (Award \# 011972-00001).

Conflict of interest. The authors have no conflicts of interest to disclose.

\section{The technical reasoning hypothesis does not rule out the potential key roles of imitation and working memory for CTC}

\author{
Alba Motes-Rodrigo $^{\mathrm{a}}{ }^{\oplus}$, Eva Reindl $\mathrm{I}^{\mathrm{b}}$ and Elisa Bandini ${ }^{\mathrm{a}}$ \\ aDepartment of Early Prehistory and Quaternary Ecology, Eberhard Karls \\ University of Tübingen, 72070 Tübingen, Germany and ${ }^{b}$ School of Psychology \\ and Neuroscience, University of St Andrews, St Mary's Quad, KY16 9JP St \\ Andrews, UK. \\ albamotes7@gmail.com \\ elisa-bandini@hotmail.it \\ eva.reindl@live.de \\ https://sites.google.com/view/alba-motes-rodrigo/home \\ https://sites.google.com/view/elisabandini/home \\ http://www.evareindl.com/
}

doi:10.1017/S0140525X20000047, e173

\begin{abstract}
To support their claim for technical reasoning skills rather than imitation as the key for cumulative technological culture (CTC), Osiurak and Reynaud argue that chimpanzees can imitate mechanical actions, but do not have CTC. They also state that an increase in working memory in human evolution could not have been a key driver of CTC. We discuss why we disagree with these claims.
\end{abstract}

Osiurak and Reynaud (O\&R) present a novel approach to the study of cumulative technological culture (CTC) by focusing on the role of technical reasoning skills rather than the traditional high-fidelity social-learning approach prevalent in the literature. The authors discuss several candidate cognitive abilities (e.g., imitation, theory of mind, and working memory (WM)) that are widely viewed as prerequisites for the emergence of CTC and have also been argued to differ between humans and other animals. The authors maintain that none of these abilities are necessary for the occurrence of CTC but instead are merely boosting elements, with technological reasoning skills being the real drivers of CTC. Here, we focus on two of these cognitive capacities: imitation and WM.

To strengthen their claim for technological reasoning being the pivotal ability supporting CTC rather than imitation and other mechanisms of the social dimension, the authors argue that chimpanzees possess imitative abilities but do not show evidence of CTC. Therefore, according to their argumentation, imitation cannot be a driving force of CTC. However, the authors have neglected to discuss crucial literature on imitation in chimpanzees that contradicts their arguments and would invalidate their conclusion about this ability in great apes. The authors base their argument that chimpanzees can imitate on the so-called "two-action tests," which originally claimed to test whether chimpanzees would imitate the mechanical actions of a demonstrator who was trained in one of two techniques to open a puzzle box (Horner \& Whiten 2005; Horner et al. 2006; Whiten et al. 2005). The finding that subjects will generally converge on the technique of the demonstrator has often been taken as evidence that chimpanzees can imitate actions. However, these tests are methodologically flawed, mainly because the two different actions often lead to two different results, making it impossible to disentangle result from action copying. Therefore, these tasks would be better named "two-action, two-results tests," as they do not allow differentiating between social-learning mechanisms. Finally, in all of these studies, subjects individually reinnovated the non-seeded action, demonstrating that these solutions are not beyond the spontaneous capabilities of each individual, and do not strictly require imitative skills to be acquired.

In fact, studies aimed at directly testing for imitation in chimpanzees have failed to find this ability (Henrich \& Tennie 2017). For instance, Tennie et al. (2012) conducted an experiment with the goal to elicit imitation of motor actions in captive chimpanzees using conspecific demonstrators. The authors found that chimpanzees showed no evidence of action imitation despite ample opportunities for observational learning (see also Tennie et al. 2010, for a different task). Similar negative results have been found across great ape species and experimental tasks (bonobos: Clay and Tennie 2017; all great apes: Tennie et al. 2009). Therefore, we disagree with the authors' interpretation of the great ape imitation literature, and encourage the authors to reassess their line of reasoning in this regard.

Regarding WM, the authors argue against the possibility that an increase in WM capacity in human evolution could have been a key driver of CTC. They claim that an increase in WM capacity could not explain why humans - but not great apes are capable of "combined mechanical actions." The authors rightly argue that "maintain[ing] multiple mechanical actions in memory for a period of time is cognitively different from being able to combine them." However, they might be wrong in assuming that WM only allows for storing mechanical actions, but not for combining them. Although no single, agreed-upon definition of WM exists, most researchers today refer to the mere temporary maintenance of information in mind as short-term memory (Cowan 2017). In contrast, WM is defined by many - including Wynn and Coolidge (2007), which are cited by the authors - as the ability to maintain and manipulate mental representations, going beyond simple storage and allowing for information processing and interrelating representations (Cowan 2017; Diamond 2013). Therefore, the authors seem to conflate the concepts of WM and short-term memory. If taken as a storage and processing capacity, WM increases could allow for combinations of mechanical actions - there might be no need to assume separate technical reasoning skills.

The authors further argue that WM could not generate content and thus technical reasoning skills would be necessary to do so. However, note that WM is thought to play a crucial role in the creation of novel procedures or tools: greater WM capacity allows one to "see novel combinations of tools and materials or look farther ahead in the procedural sequence to see novel consequences" (Wynn \& Coolidge 2014). 
One might even argue that technical reasoning itself requires a considerable amount of WM to evolve. For example, the authors suggest that the "outcome of technical reasoning is a mental simulation of the mechanical action [...] and this simulation needs to be temporarily maintained." WM - by definition - is precisely the platform needed for storing and simulating such mental content. Second, the authors describe technical reasoning as being "analogical." Analogical reasoning itself (potentially also in the technical domain) relies critically on WM, as it requires one to hold multiple representations and their relational information in mind (Halford et al. 1998; Simms et al. 2018). Thus, the authors' arguments against the role of WM for CTC seem weak. WM as a potential key factor for CTC should not be prematurely ruled out.

In conclusion, we agree with the authors that the role of technological reasoning skills in CTC has been underestimated, and the role of imitation perhaps overestimated. However, by neglecting to engage with the most recent developments in the field of great ape social learning, and by basing some of their conclusions about WM on weak arguments, the authors' current formulation of the technical reasoning hypothesis loses some credibility. Despite this, we applaud their efforts toward questioning the status quo of the literature on the cognitive mechanisms behind CTC, and look forwards to reading about experimental applications of their hypothesis.

Financial support. This work was supported by the European Research Council (ERC) under the European Union's Horizon 2020 research and innovation program (AMR, grant agreement no. 714658); and the Institutional Strategy of the University of Tübingen (EB, Deutsche Forschungsgemeinschaft, ZUK 63).

Conflict of interest. None.

\section{A long view of cumulative technological culture}

\section{Michael J. O'Brien ${ }^{\mathrm{a}}{ }^{\circ}$ and R. Alexander Bentley ${ }^{\mathrm{b}}$}

\author{
a Department of Communication, History, and Philosophy, Texas A\&M \\ University-San Antonio, San Antonio, TX 78224 and ${ }^{\text {b Department of }}$ \\ Anthropology, University of Tennessee, Knoxville, TN 37996. \\ mike.obrien@tamusa.edu \\ rabentley@utk.edu
}

doi:10.1017/S0140525X20000060, e174

\begin{abstract}
We agree that the emergence of cumulative technological culture was tied to nonsocial cognitive skills, namely, technicalreasoning skills, which allowed humans to constantly acquire and improve information. Our concern is with a reading of the history of cumulative technological culture that is based largely on modern experiments in simulated settings and less on phenomena crucial to the long-term dynamics of cultural evolution.
\end{abstract}

Learning and cognition have become increasingly important components of archeology - our discipline - as researchers have begun to integrate cultural evolutionary theory as a framework for explaining the archeological record (Shennan 2008; Stout et al. 2019). Significant strides have been conducted over the past two decades in understanding how the results of small-scale evolutionary processes, when taken in the aggregate, can create population-level patterns seen in the record of human origins and spread, first out of Africa and across Europe and Asia (Bae et al. 2017), then eventually into Australia (Clarkson et al. 2017), and finally, within the last 15,000 years, into the Americas (O'Brien 2019). Fundamental to this framework has been an emphasis on two kinds of learning, social and individual (O'Brien \& Buchanan 2017). Certain conditions, perceived or real, dictate which type of learning might be more useful in any particular situation (Mesoudi 2008; 2010).

Many animals have the ability to learn socially (Brown \& Laland 2003; Heyes \& Galef 1996; Hoppitt \& Laland 2013; Watson et al. 2018; Whitehead \& Rendell 2015; Whiten \& van de Waal 2017), but because of their extremely high phenotypic plasticity, humans have the unique and highly useful ability to learn from others exactly how and when to learn from others what Mesoudi et al. (2016) refer to as "the social learning of social learning." We see the outcome of this ability manifest in the archeological record as cultural traditions (Willey \& Phillips 1958) - temporal continuity represented by persistent configurations in single technologies or other systems of related forms that result from cultural transmission.

Osiurak and Reynaud (O\&R) argue that the cognitive mechanisms underlying individual and social learning are insufficient for explaining the emergence of cumulative technological culture and that it originated in nonsocial cognitive skills, namely, technical-reasoning skills that allowed humans to constantly acquire and improve information. We do not think this view is particularly controversial, and in fact, we think they are probably correct, although we would argue that this revised assumption in no way allows us to ignore the social dimension and to redirect our focus to the nonsocial, strictly technical dimension. Rather, our principle concern is with a reading of the history of cumulative technological culture that is based largely on modern experiments in simulated settings. In prehistory, a population comprised people who lived in a region, with a full demographic distribution of ages and kinship relationships, and, most importantly, millennia of shared ancestral history of cohabitation, cooperation, specialization, migration, reproduction, adaptation, cultural selection, and sorting.

These phenomena are crucial to the long-term dynamics of cumulative cultural evolution. In many of the experiments reviewed by O\&R, only a tiny aspect of these dynamics - momentary social learning in a very specific experiment - is represented, and in an unnatural setting. Consider, for example, the vast difference between an undergraduate psychology student spending two minutes studying a paper airplane built in 5 minutes by another undergraduate student versus a Paleolithic boy learning how to knap stone tools over his entire childhood on a daily basis, in a range of different settings. The boy learns repeatedly by observing a parent and other kin members, young and old, all of whom are replicating a tradition that evolved over centuries and millennia of intergenerational transmission.

This type of behavior is difficult if not impossible to replicate in an experiment, so at the very least experimenters need to carefully consider many questions to gain any insight on cumulative culture. For psychologists and evolutionists to reconcile their scales of analysis, they need to ask: What was the context of this learning? How different is learning by studying a paper 
plane for two minutes versus regular, repeated practice over years of childhood, of a task that was fundamentally embedded in kin relationships, survival, and relationship with the environment? What was the significance of learning repeatedly from different generations of kin for all those years? What other knowledge had to be learned at the same time? Even as we consider all these factors in one generation and one individual, we need to iterate this day-in-day-out process for dozens or even hundreds of generations, cumulatively thousands of people, all of whom were influenced by other cultural evolution factors.

These are the real details of the "evolution" part of cumulative cultural evolution (Mesoudi \& Thornton 2018). Yes, a transmission-chain experiment can be designed to include elements of variation and transmission and selection of sorts, but it might nevertheless be highly contrived by the experiment design. What aspects of evolutionary success would these shortterm, laboratory measures miss regarding, say, the evolution of prehistoric projectile points or fishing hooks over many centuries? The differences between real-technological evolution and psychology experiments include many things, including the success of intergenerational transmission and success across multiple tasks.

We do not negate the usefulness of the kinds of micro-society paradigms discussed by $\mathrm{O} \& \mathrm{R}$ for understanding the role of cognitive skills and/or the quality of social transmission under certain conditions. In fact, similar to other archeologists (e.g., Schillinger et al. 2014), we have conducted such experiments ourselves to better understand certain learning processes that left particular signatures in the archeological record (Atkinson et al. 2012; Mesoudi \& O'Brien 2008). However, we agree with Caldwell et al. (2019, p. 12) that "empirical investigation has been impeded by the methodological difficulty of studying a population-level phenomenon using [only] experimental methods."

In summary, it no longer is productive simply to substitute the word "population" for a group of students, "culture" for a momentary, contrived task learned in an experiment, or "generation" for passing the task to the next student. None of this is trivial or just semantic; rather, it is crucial to the process of cumulative cultural evolution. We suspect that the next significant breakthrough in understanding cumulative culture will need to directly address multiple issues, including the effect of lifelong learning within a kin group, multigenerational transmission over centuries of human adaptation, and realistic demography. This must be done critically through theory, model, and experiment (Mesoudi \& Whiten 2008).

Financial support. This research received no specific grant from any funding agency, commercial, or not-for-profit sectors.

Conflict of interest. None.

\section{The social side of innovation}

\section{Bruce Rawlings (1) and Cristine H. Legare (1)}

Department of Psychology, University of Texas at Austin, Austin, TX 78712. bruce.rawlings@utexas.edu chlegare@gmail.com

https://www.brucerawlings.com/ http://www.cristinelegare.com/

doi:10.1017/S0140525X20000217, e175

\section{Abstract}

Innovation is fundamental to cumulative culture, allowing progressive modification of existing technology. The authors define innovation as an asocial process, uninfluenced by social information. We argue that innovation is inherently social innovation is frequently the product of modifying others' outputs, and successful innovations are acquired by others. Research should target examination of the cognitive underpinnings of socially-mediated innovations.

Osiurak and Reynaud $(\mathrm{O} \& \mathrm{R})$ provide a novel and interesting perspective on the cognitive basis of cumulative technological culture (CTC). They argue that humans' enhanced capacity for technicalreasoning - the ability to reason about the causal function of physical objects - is the driving force behind CTC, providing the causal knowledge needed for cumulative technological development. The paper highlights the need for a cognitive approach to understanding cumulative culture, with a particular focus on technical (causal) reasoning. The article presents a detailed account of how technical-reasoning underpins human tool innovation - which in turn facilitates CTC.

Innovation has been extensively studied across multiple disciplines, including, among others, psychology, archeology, anthropology, zoology, business studies, and economics. Measures of innovation vary across disciplines, from individual problemsolving challenges to accruement of patents and industry awards. The authors, in using Ramsey et al.'s (2007) definition, adopt the traditional cultural evolution approach to innovation, in which it is treated as an exclusively asocial process, devoid of influence from social information; "Innovation can be defined as the generation of a novel learned behavior that is not the consequence of social learning or environmental induction (Ramsey et al. 2007)" (sect. 2.2.4, para. 1).

Although there is some variation in definitions of innovation across fields, it is typically required that an innovation should be novel and valuable (criterion used in the definition of the target article). Innovations should also be transmitted to others, a social component, as a marker of their value (Muthukrishna \& Henrich 2016), a criterion not applied by O\&R.

We, thus, comment on the definition of innovation used, and suggest that O\&R's account would be strengthened by considering the contribution social learning makes to innovation at the individual and group levels. Specifically, explicitly distinguishing individual-level asocial innovations from socially-mediated innovations - a distinction gaining increasing traction - would help understand how each form contributes to cumulative culture. This would also allow investigation of whether technicalreasoning underpins independent invention and innovations based on modifying others' products. Additionally, because cumulative culture depends upon innovations being faithfully transmitted to others, researchers should consider the wider social contexts involved in the successful uptake of innovations by others. Throughout our commentary, we focus on the development of tool innovation to illustrate our points.

Innovation can be investigated at both individual and group levels. At the individual level, for instance, developmental psychologists examining the ontogeny of tool innovation typically assess this through the presentation of a novel puzzle which participants must individually solve. A widely used example is the "Hook Task," whereby children must fashion a pipecleaner into 
a hook shape to retrieve an out of reach reward (Beck et al. 2016). Arguably, the most notable result from previous research using this task is that children, across cultures, find asocially solving this relatively simple problem markedly difficult. Performance improves with age, but it is not until around early adolescence when the majority children consistently solve these types of asocial tool-use problems.

Yet, asocial problem-solving tasks by nature prohibit investigation of socially-mediated innovations. There has been a recent shift within cultural evolution studies to incorporate the role of social information during some (potentially most) innovations (Carr et al. 2016; Muthukrishna \& Henrich 2016). This conceptualization delineates asocial innovation (termed innovation-by-invention), from innovations arising from modifying, combining, or refining previously witnessed tool-behaviors (termed innovation-by-modification), such as an incorrectly-sized hook (Cutting et al. 2019). The former, which may be relatively rare, might arise from particularly gifted individuals or serendipitous events, whereas the latter, likely to be more frequent, involves modifying the behaviors or output of others, thereby incorporating some form of social learning.

The distinction between asocial- and socially-mediated innovations is necessary because each may differentially contribute to cultural evolution and thus to CTC (Carr et al. 2016). Innovation-by-invention forms the basis for diversification of cultural technology, whereas cumulative technological progression depends upon the modification of existing technology. That is, technological advances are typically not the product of a sole inventor, but through incremental modifications of others' output. Experimental work has begun to indicate that each form of innovation may have different developmental trajectories (Carr et al. 2015; Cutting et al. 2019), potentially signifying they may be supported by different cognitive mechanisms. Thus, we encourage $\mathrm{O} \& \mathrm{R}$ to delineate innovations arising from asocial invention and those based on modifying the output of others. This would also allow further investigation of the developmental trajectory and cognitive underpinnings (including the role technical-reasoning plays) in each form of innovation.

At the group level, there is a need to examine how innovations spread throughout populations.

Humans' vast cultural diversity is because of our capacity to acquire and transmit innovations (Legare \& Nielsen 2015). Cumulative technological progression is contingent on the social transmission of novel behaviors, and successful diffusion throughout a population is indicative of an innovation's value, and the producer's sense of others' need for innovations. Thus, innovations are inherently social and should be examined as such (Turner \& Flynn 2016). To fully understand human cumulative culture, there is a need to examine, in unison, asocially- and socially-mediated innovations, and their subsequent social diffusion. Psychologists are now well placed to employ experimental and observational techniques to examine how individuals perceive and adopt novel behaviors from others, group level characteristics more conducive to successful diffusion, and the cognitive mechanisms involved in the successful uptake others' innovations.

Human CTC is fundamental to our species' cultural diversity; diversity purportedly maintained through social learning and innovation. Innovation has traditionally been represented as independent invention, and research has targeted determining characteristics of so-called innovators and the contexts which promote successful innovation. Yet, in contrast to claims that innovation is an asocial endeavor, we argue that innovation is inherently social - it typically involves modification of the outputs of others and successful innovations are socially transmitted to others. Both the individual and group approaches to examining innovation are interesting and crucial avenues of investigation. The more these approaches are integrated, the more scientific value they hold.

Financial support. This work was supported by the National Science Foundation (grant number 1730678).

Conflict of interest. None.

\section{A little too technical: The threat of intellectualising technical reasoning}

\section{Ian Robertson (1)}

Faculty of Law, Humanities and the Arts, University of Wollongong, Wollongong, NSW 2522, Australia.

ianrob@uow.edu.au

\section{doi:10.1017/S0140525X20000242, e176}

\begin{abstract}
Osiurak and Reynaud (O\&R) claim that research into the origin of cumulative technological culture has been too focused on social cognition and has consequently neglected the importance of uniquely human reasoning capacities. This commentary raises two interrelated theoretical concerns about O\&R's notion of technical-reasoning capacities, and suggests how these concerns might be met.
\end{abstract}

What is it about human cognition that has enabled us as a species to continue to sophisticate our tools and how effectively we go about using them to accomplish our everyday endeavours? Osiurak and Reynaud (O\&R) contend that research into the cognitive origins of cumulative technological culture (CTC) - the continual improvement in human tool use over generations has, to its serious detriment, been overwhelmingly focused on the notion that skilled tool practices are transmitted socially (through e.g., teaching and imitation). This focus on the social dimension of CTC has, they claim, lead researchers to underestimate the importance of uniquely human-reasoning capacities in explaining its cognitive genesis.

O\&R appeal to a wide range of empirical data to support what they refer to as the technical-reasoning hypothesis: the contention that CTC "originates in non-social cognitive skills, namely technical-reasoning skills which enable humans to develop the technical potential necessary to constantly acquire and improve technical information" (abstract). Questions might reasonably be asked as to what O\&R take technical information to be, but I want to focus here on their characterisation of technical-reasoning capacities. For them, technical reasoning is "the necessary cognitive structure that enables humans to constantly acquire and develop new [tool incorporating] techniques" (sect. 1, para. 4). They claim that it "starts by using mechanical knowledge in order to find a physical principle that is thought to be [...] appropriate for solving the targeted physical problem (e.g., cutting) as well as the associated physical object properties (i.e., sharp, etc.)" (sect. 2.1, para. 4, item 3). An issue that immediately arises for the technical-reasoning hypothesis is what the nature of the 
thought and reasoning capacities involved in technical reasoning is supposed to be.

This commentary raises two interrelated theoretical concerns about O\&R's technical-reasoning hypothesis. The first is that their notion of mechanical knowledge is underdefined and perhaps incoherent; the second is that their characterisation of technical reasoning as a kind of Bayesian learning potentially undermines their key claim that technical reasoning is uniquely human. Each of these worries pertains to a more fundamental concern: namely that O\&R's account of the cognitive origins of CTC seem to rely on an overly-intellectualised characterisation of the cognitive activity involved in skilled tool practices.

For O\&R, mechanistic knowledge is "a kind of magic" (sect. 2.3). It is, more specifically, a kind of non-declarative "knowledge about physical principles" (sect. 2.1, para. 4, item 2), which they identify with Stephen Pinker's (2010) notion of know-how. Mechanical knowledge, for them, like "any form of knowledge, is a belief but not necessarily true" (sect. 2.3, para. 1). O\&R readily allow that false beliefs - e.g., those about physical objects held by infants - can constitute knowledge providing they are "useful for inferring potential outcomes in the environment" (sect. 2.3, para 2.). Philosophers will be curious to know how it is possible to reduce knowledge to belief, given that they have been trying unsuccessfully for centuries to reduce knowledge to a kind of true belief. Setting the issue of whether knowledge is factive to one side, we should here recognise that philosophers of mind and cognitive scientists regularly posit procedural knowledge that does not reduce to knowledge of facts precisely because it is difficult to conceive of the know-how involved in paradigmatic instances of skilled sensorimotor action such as fluid tool use (e.g., competent stone knapping) as straightforwardly a matter of grasping certain facts about how to act (Fridland 2017). A possible challenge for $\mathrm{O} \& \mathrm{R}$ is to offer compelling reasons as to why the mechanistic knowledge involved in skilled tool use is best construed not as a dispositional competence but as a kind of conceptual, propositional knowledge, namely "a physical principle that is thought to be [...] appropriate for solving the targeted physical problem" (sect 2.1, para. 4, item 3). As proponents of embodied cognition emphasise, that a system's behaviour is reliably attuned to certain physical principles does not entail that it knows or represents said principles (Colombetti 2017).

Another potential problem emerges when we consider how, $q u a$ the technical-reasoning hypothesis, O\&R take mechanical knowledge to be produced and honed. They claim that an agent's proficiency in using a tool increases through practice as she gleans progressively more accurate mechanical knowledge. Over time, technical reasoning leads to inaccurate beliefs being "invalidated through a kind of Bayesian learning" (sect. 2.3, para. 2). This form of Bayesian learning is presumably not deliberate or explicit, but O\&R offer no details whatsoever about how technical reasoning is supposed to implement or conform to Bayesian norms. The notion of sub-personal cognitive activity somehow implementing Bayesian inference is increasingly popular in fields such as computational neuroscience (see Clark 2013), but researchers usually claim that the human brain approximates, rather than genuinely implementing Bayesian inference. Some researchers question whether we can really describe cognitive activity approximating Bayes as an inferential procedure at all (rather than one that unfolds as though it followed certain norms see, e.g., Bowers \& Davis 2012). But, there is a more pertinent point here for the technical-reasoning hypothesis.
Technical reasoning is characterised by O\&R as an intelligent enterprise in virtue of its amounting to a form of Bayesian learning. Recent research into the free-energy principle, however, claims that the behaviours of non-human animals in constructing their environmental niche (and thereby avoiding states with high surprisal), will, on average and over time, approximate a kind of Bayesian belief optimisation (Friston 2018). Indeed, even nonbiological systems might reasonably be construed as approximating a form of Bayesian inference (Kirchhoff \& Robertson 2018). It is therefore difficult to see how technical-reasoning capacities are, in virtue of their cohering to Bayesian norms at least, uniquely human. But, this means that technical reasoning might not be able to explain our uniquely human tool making/using capacities. A challenge going forward, then, is to better detail how the Bayesian credentials of technical reasoning facilitate a uniquely human capacity.

In sum, O\&R should be praised for their robust defence of the claim that CTC is too focused on social cognition, but they face clear theoretical challenges in characterising technical reasoning.

Financial support. This work was supported by the Australian Research Council Discovery Project "Minds in Skilled Performance" (DP170102987).

Conflict of interest. None.

\section{New Caledonian crows afford invaluable comparative insights into human cumulative technological culture}

\section{Christian Rutz ${ }^{\mathrm{a}, \mathrm{b}} \odot$ and Gavin R. Hunt ${ }^{\mathrm{c}}$}

\begin{abstract}
${ }^{a}$ Centre for Biological Diversity, School of Biology, University of St Andrews, St

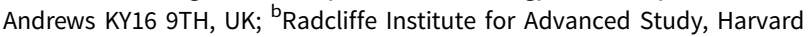
University, Cambridge MA 02138 and ' Unaffiliated.

christian.rutz@st-andrews.ac.uk grhunt10@hotmail.com

https://aviantooluse.org
\end{abstract}

\section{doi:10.1017/S0140525X20000187, e177}

\section{Abstract}

The New Caledonian crow may be the only non-primate species exhibiting cumulative technological culture. Its foraging tools show clear signs of diversification and progressive refinement, and it seems likely that at least some tool-related information is passed across generations via social learning. Here, we explain how these remarkable birds can help us uncover the basic biological processes driving technological progress.

Cumulative culture - the social transmission and accumulation of innovations across generations - has enabled humans to develop technologies of astonishing complexity (Boyd \& Richerson 1996). Osiurak and Reynaud (O\&R) present a timely re-evaluation of the potential cognitive capacities enabling this process, shifting the focus from social-learning to technical-reasoning skills. We enjoyed O\&R's fresh perspective, but were surprised that they did not examine more carefully their starting assumption that only humans possess cumulative technological culture. Two non- 
human species have been suggested to refine their foraging tools in a cumulative manner - one of our closest relatives, the chimpanzee, and a tropical corvid, the New Caledonian crow (Dean et al. 2014). Although O\&R mention New Caledonian crows in passing, we feel they have missed a valuable opportunity to consult the large body of evidence available for this non-primate model system. Here, we provide some of this missing context, and argue that debates about cumulative technological culture benefit greatly from the comparative insights afforded by these remarkable birds.

According to a recent consensus definition (Mesoudi \& Thornton 2018), the New Caledonian crow is a compelling candidate for cumulative technological culture (Hunt \& Uomini 2016; St Clair et al. 2018). A detailed evaluation is beyond the scope of this commentary and will be provided elsewhere, but the key points can be summarised as follows. First, New Caledonian crows' technology exhibits clear signs of cumulative refinement. In addition to using unmodified sticks and other objects, the species actively manufactures two elaborate tool types from living plant material: hooked stick tools and barbed leaf tools (Hunt 1996; Rutz \& St Clair 2012). For both of these, distinct variants have been described that strongly suggest past accumulation of design innovations, progressing from basic to increasingly complex tools (Hunt \& Gray 2003; St Clair \& Rutz 2013). Second, controlled experiments have recently demonstrated that hooked stick tools are much better for extracting prey across a variety of foraging scenarios than (the presumably ancestral) non-hooked stick tools (St Clair et al. 2018), and that well-crafted, deep hooks are particularly efficient (Sugasawa et al. 2017). Third, it seems likely that at least some toolrelated information is passed across generations via social learning, leading to a rudimentary form of material culture, with striking regional variation in tool behaviour (Hunt \& Gray 2003; St Clair et al. 2016; see also Bluff et al. 2010a). Here, the evidence is weaker (review: Rutz et al. 2018), but field studies have shown that juvenile crows hone their tool-related skills during an unusually long dependency period (Bluff et al. 2010b; Hunt et al. 2012), which affords abundant opportunities to observe experienced adults (e.g., Holzhaider et al. 2010a; Rutz et al. 2012; St Clair et al. 2015), and laboratory experiments have confirmed a capacity to learn both from demonstrators (Kenward et al. 2006; Logan et al. 2016) and artefacts (Jelbert et al. 2018).

O\&R identified technical-reasoning skills as a potential cognitive prerequisite of cumulative technological culture. New Caledonian crows' tool repertoire is obviously rudimentary compared to human technology, consisting entirely of one-piece artefacts crafted from plant materials, but the system allows us to chart the minimal cognitive capacities required for the accumulation of design improvements. Some 20 years of research on the species' tool-related cognition provide instructive insights. New Caledonian crows have been shown: to make and/or select task-appropriate tools (e.g., Bluff et al. 2007; 2010b; Chappell \& Kacelnik 2002; 2004; Knaebe et al. 2017), most likely relying on simple "heuristics" (Hunt et al. 2006; G.R. Hunt, unpublished data); to attend to different tool design features (Knaebe et al. 2015; St Clair \& Rutz 2013; but see Holzhaider et al. 2008); and to learn quickly - and even invent - novel tool behaviours (Rutz, unpublished manuscript). Although these skills are impressive, reports of more advanced cognitive abilities have routinely been contested. This includes claims that New Caledonian crows are capable of: tool-related causal reasoning (pro: Taylor et al. 2011; contra: Ghirlanda \& Lind 2017; Hennefield et al.
2018); analogical reasoning in the context of sequential tool use (pro: Taylor et al. 2007; contra: Wimpenny et al. 2009; and see: Taylor et al. 2010); making inferences about hidden causal agents (pro: Taylor et al. 2012b; contra: Boogert et al. 2013; Dymond et al. 2013); creating novel causal interventions (pro: Jacobs et al. 2015; contra: Taylor et al. 2014; and see: von Bayern et al. 2009); adaptively fashioning new tool shapes (pro: Weir et al. 2002; contra: Rutz et al. 2016b); and using mental representations to solve tool problems (pro: Gruber et al. 2019; contra: Vonk 2019). These disagreements may partly reflect the methodological challenges inherent in probing the minds of non-verbal subjects, but in our view, they also bring into focus the upper limits of the species' reasoning abilities.

In conclusion, New Caledonian crows excel at solving toolrelated tasks, exhibiting a clear capacity for quick learning and behavioural flexibility, but evidence of more advanced cognitive abilities is much weaker. This suggests that a rudimentary form of cumulative technological advancement is possible without some of the technical-reasoning skills O\&R predicted to be essential (as well as without language and active teaching; see Bluff et al. 2010a; Holzhaider et al. 2010a). If our preliminary assessment is correct, it would raise the possibility that cognitive constraints are currently limiting the scope for further technological advancement in New Caledonian crows, and/or that different mechanisms enable cumulative technology in humans and New Caledonian crows. Although non-cultural processes are expected to contribute to shaping the technology of New Caledonian crows (Kenward et al. 2005; Rutz et al. 2018), we should not underestimate the extent to which this was also the case in our tool-making ancestors (e.g., Corbey et al. 2016). Therefore, whatever the precise contributions of genetics, individual learning and social transmission (Hunt \& Uomini 2016; Rutz et al. 2018), the New Caledonian crow - and other non-human tool users (e.g., Rutz et al. 2016a) - provide a powerful comparative framework (Dean et al. 2014) for identifying the basic biological processes that enable cumulative technological evolution.

Acknowledgments. We thank Barbara Klump, Phil Shaw and Matthew Steele for constructive comments.

Financial support. C.R. drafted this commentary while he was the grateful recipient of a Radcliffe Fellowship at the Radcliffe Institute for Advanced Study, Harvard University, USA. G.R.H.'s contributions to the development and editing of the manuscript were self-funded.

Conflict of interest. None.

\section{The crow in the room: New Caledonian crows offer insight into the necessary and sufficient conditions for cumulative cultural evolution}

\section{Alex H. Taylor ${ }^{\mathrm{a}}$ (1) and Sarah Jelbert ${ }^{\mathrm{b}}$}

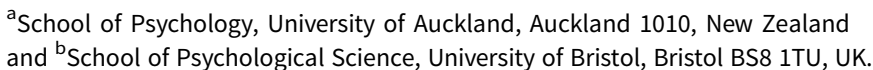


Alexander.taylor@auckland.ac.nz

Sarah.jelbert@bristol.ac.uk

https://www.psych.auckland.ac.nz/en/about/animal-minds.html

http://www.bris.ac.uk/expsych/people/sarah-a-jelbert/overview.html

doi:10.1017/S0140525X20000102, e178

\begin{abstract}
New Caledonian (NC) crow populations have developed complex tools that show suggestive evidence of cumulative change. These tool designs, therefore, appear to be the product of cumulative technological culture (CTC). We suggest that tool-using NC crows offer highly useful data for current debates over the necessary and sufficient conditions for the emergence of CTC.
\end{abstract}

The tools of New Caledonian (NC) crows offer some of best evidence for cumulative cultural evolution in non-human animals (Hunt \& Gray 2003). These birds manufacture tools from a range of materials for extractive foraging and other purposes (Hunt 1996; St-Clair et al. 2018; Taylor et al. 2012a; 2012b; Wimpenny et al. 2011), and show considerable diversity in tool design across different populations, which cannot be explained by ecological conditions alone (Hunt \& Gray 2003). Some wild NC crows incorporate hooks into their tool designs, a level of technology not seen in our closest relatives, the chimpanzee, and that appears to have only emerged in our species around 100 kya (Yellen et al. 1995). In particular, NC crows incorporate naturally occurring barbs into the leaf tools they make from pandanus plants (Hunt 1996; 2000). These pandanus tools are particularly impressive because of their stepped design: crows cut steps into the edge of pandanus leaf tools as they fashion them, with the number of steps cut into the leaf edges showing large variation between populations. It is this variation in tool designs that provides strong evidence for cumulative cultural. These tool designs show evidence of cumulative improvement between populations, are not created by naïve juveniles and the variation between populations is not explained by ecological correlates (Hunt 2000; Hunt \& Gray 2003; Kenward et al. 2005).

However, work on this bird species has failed to find evidence for many of the social cognitive mechanisms that have been claimed to be required for cumulative technological culture (CTC), such as imitation, teaching, and language (Jelbert et al. 2018; Logan et al. 2016). For example, Logan et al. (2016) presented a classic two-action task to NC crows, but found evidence only of stimulus enhancement and individual trial and error learning, rather than evidence of imitation. There is also currently little evidence for metacognition or theory of mind in this species (Taylor 2014).

Instead, recent work suggests that CTC in this species is best explained by a form of reverse engineering (or goal emulation), which we termed "mental template matching" (Jelbert et al. 2018; Logan et al. 2016). We have suggested that crows form a mental template of a particular tool design from observing or using tools made by conspecifics, and then reverse engineer this design when they manufacture their own tools. A recent paper-ripping study provides good evidence that NC crows have the cognitive capacity for mental template matching (Jelbert et al. 2018). Crows that learned that either pre-made large or small pieces of card could be inserted into a vending machine to obtain food rewards, subsequently manufactured pieces of card that matched the sizes of the previously rewarded examples. This behaviour occurred even though subjects were rewarded at random for the items they manufactured at test, and there was no available tool design to copy, meaning only the crow's mental representation of the correct tool design could guide manufacture. The presence of this cognitive capacity, coupled with observations showing adult-like pandanus manufacture develops slowly over the first year of life (Holzhaider et al. 2010b), that juveniles regularly borrow their parents tools (Holzhaider et al. 2011), and the parallels between template matching and song bird learning (Jelbert et al. 2018; Slater 1983) provide strong support for the hypothesis that $\mathrm{NC}$ crows pass on and update tool designs through reverse engineering in the absence of advanced social cognition.

Importantly, although NC crows do not appear to have remarkable social cognition (Jelbert et al. 2015a; 2015b; Logan et al. 2016), their technical reasoning abilities are substantial and have been the focus of much research. In line with Osiurak and Reynaud's (O\&R) technical reasoning hypothesis, there is good evidence for a range of different types of technical intelligence in this species. NC crows show evidence for the ability to transfer causal knowledge between perceptually distinct problems (Taylor et al. 2007; 2009), though it is not clear if this is via functional generalisation or true analogical reasoning. They also can mentally preplan solutions to problems (Gruber et al. 2019) and make inferences, such as reasoning by exclusion (Jelbert et al. $2015 \mathrm{a} ; 2015 \mathrm{~b})$ and inferring the weight of novel objects from observing their movements in the wind (Jelbert et al. 2019). There is also some evidence that NC crows might be capable of representing hidden causal mechanisms (Taylor et al. 2012a; 2012b; but see Boogert et al. 2013; Dymond et al. 2013; Taylor et al. 2013a; 2013b), but there is currently no conclusive evidence that they form representations of unobservable ones (Neilands et al. 2016; Taylor et al. 2009), nor that these birds possess the ability to make causal interventions (Taylor et al. 2014; but see Jacobs et al. 2015; Taylor et al. 2015).

This pattern of results provides strong general support for the technical reasoning hypothesis raised by O\&R. NC crows show a profile of high technical reasoning abilities, less developed social intelligence, yet strong indications of CTC. This profile is in line with the hypothesis that CTC initially arises because of technical intelligence, rather than social intelligence. However, it also suggests that not all the technical reasoning skills of humans are necessary and sufficient for CTC. Work on NC crows suggests that CTC can emerge not only in the absence of imitation, teaching, and language, but also in the absence of analogical reasoning, reasoning about unobservable causal mechanisms, and possibly also the ability to make causal interventions. Instead, it appears that a more limited causal understanding, along with functional generalisation, inference, and mental simulation, might be a sufficient suite of technical intelligence skills for cumulative cultural evolution to emerge via template matching. Clearly though, far more work is needed to test this hypothesis further. This is not to say that many other abilities are not required for the full complexity of the cumulative cultural evolution we currently see in humans, but that the necessary and sufficient cognitive requirements for this process to start in a species might be simpler than the full suite of technical reasoning skills O\&R suggest.

Financial support. None.

Conflict of interest. None. 


\section{Supporting the weight of the elephant in the room: Technical intelligence propped up by social cognition and language}

\author{
Alex Thornton ${ }^{a} \odot$, Francesca Happéb ${ }^{(1)}$ \\ and Christine A. Caldwellc ${ }^{\circ}$
}

${ }^{a}$ Centre for Ecology and Conservation, University of Exeter, Penryn TR10 9FE, UK; ' Social, Genetic, and Developmental Psychiatry Centre, Institute of Psychiatry, Psychology, and Neuroscience, King's College London, London SE5 8AF, UK and 'Department of Psychology, University of Stirling, Stirling FK9 4LA, UK.

alex.thornton@exeter.ac.uk

c.a.caldwell@stir.ac.uk

francesca.happe@kcl.ac.uk

http://www.wildcognitionresearch.com/

https://sites.google.com/site/christineannacaldwell/

https://www.kcl.ac.uk/people/francesca-happe

doi:10.1017/S0140525X20000114, e179

\begin{abstract}
We consider the evolutionary plausibility of Osiurak and Reynaud's (O\&R) arguments. We argue that technical reasoning is not quite the magic bullet that $\mathrm{O} \& \mathrm{R}$ assume, and instead propose a co-evolutionary account of the interplay between technical reasoning and social learning, with language emerging as a vital issue neglected in O\&R's account.
\end{abstract}

For decades, high-fidelity social-learning mechanisms such as imitation and teaching have been touted as the fundamental pre-requisites for cumulative culture (Boyd \& Richerson 1996; Tomasello et al. 1993). Osiurak and Reynaud (O\&R) provide a useful corrective to this narrative, instead highlighting technical reasoning as the overlooked "elephant in the room." We are largely sympathetic to this argument - indeed our own transmission chain experiments demonstrate that cumulative improvements in tools can occur in the absence of opportunities for teaching and imitation (Caldwell \& Millen 2009; Zwirner \& Thornton 2015). However, we are sceptical that technical reasoning represents a magic bullet for understanding cumulative culture.

First, O\&R's account is evolutionarily implausible, as it assumes that ancestral hominins must have made a giant cognitive leap to evolve modern technical reasoning skills before cumulative culture could get off the ground. O\&R provide no suggestions as to what selective pressures might drive the evolution of technical reasoning in ancestral populations. If we are to understand the origins of human cumulative culture, a focus on proximate cognitive mechanisms must go hand-in-hand with attempts to understand ultimate, functional drivers.

Second, by definition, culture of any kind (including cumulative culture) requires social learning (Boyd \& Richerson 1985; Mesoudi \& Thornton 2018). No account of the origins of cumulative culture can therefore be complete without consideration of how learned behaviour can be transmitted between individuals. O\&R suggest that technical reasoning skills may themselves provide the cognitive foundations for imitation and teaching, but this again leaves open the question of how and why technical reasoning skills themselves originated. It also downplays the fact that social learning does pose specific cognitive challenges, not least the need for learners to direct their attention towards potentially profitable sources of social information (Kendal et al. 2018). In both humans and many non-human animals, social learning plays a vital role in the development of many fundamental aspects of behaviour, including parental care, foraging, communication and social conventions (Thornton \& Clutton-Brock 2011; Whiten 2019a). Thus, it is likely that social learning mechanisms pre-dated and indeed scaffolded the later (genetic and/or cultural) evolution of human-like technical reasoning skills. Indeed, evidence suggests that the acquisition of technical skills in children is shaped and guided by social learning from experienced adults (Beck et al. 2011; Nielsen 2013).

The core of O\&R's argument is that "cumulative technological culture originates in non-social cognitive skills" (our emphasis). A more plausible suggestion is that the sensory, motoric and cognitive mechanisms that underpin technical reasoning co-evolved with social learning in a positive feedback loop as human populations became increasingly reliant on tools and technologies to access resources following our ancestors' invasion of the savannas (Caldwell et al. 2018; Morgan et al. 2015; Zwirner \& Thornton 2015). There is compelling evidence that relatively simple learning processes can allow groups of animals to reach optimal solutions to problems that would be difficult for a single individual (Saldana et al. 2019; Sasaki \& Biro 2017). Thus, the core criteria for cumulative cultural evolution - that is sequential improvements in the performance of behaviour over multiple episodes of social transmission (Mesoudi \& Thornton 2018) - are likely to be present in some non-human animals. Processes similar to those found in other species may well have led to modest, cumulative improvements in early hominin tools. As our ancestors' reliance on tools increased, so too would the benefits of mechanisms to understand how tools work and to transmit that information socially, in turn facilitating the production of more complex and effective tools. Consistent with this argument, evidence shows that teaching provides no clear advantage over other means of social learning in tasks involving structurally simple tools, but begins to provide important advantages as tools become more complex (Caldwell et al. 2018; Zwirner \& Thornton 2015). The mutually reinforcing advantages of teaching and technical reasoning could therefore ratchet up, eventually opening up entirely new design spaces that could never have been reached without prior episodes of innovation and cultural transmission. Thus, although all putative examples of cumulative cultural evolution in other species are restricted to specific contexts where there is a single, fixed optimum behaviour (for instance, finding the most direct route between two points: Sasaki and Biro 2017), the scope of human culture is open-ended, with cultural traits that can continually change, recombine and diversify.

The idea that teaching may have played an increasingly important role in human cultural transmission brings us to a vital element that is largely overlooked in O\&R's account: language. Critically, language allows us to teach in an almost infinite range of contexts, to pass on information about what not to do (which is all but impossible to extract through reverse engineering alone), and to request specific information when needed. This allows the scope of human teaching to surpass anything seen in other species, where teaching is restricted to specific, species-typical adaptive behaviours such as hunting (Thornton 
\& Raihani 2008). If the origins of language lie in the need to teach hard-to-learn skills to kin, as suggested by Laland (2017b), it is also likely that the evolution of linguistic skills would have altered the selective environment for other cognitive traits. Thus, as our ancestors' linguistic skills evolved, so too would the benefits of being able to represent, and convey information about, unobservable mental states and physical properties, eventually enabling targeted social transmission of technical knowledge from knowledgeable to naïve individuals.

The challenge for students of cumulative culture is two-fold: we must both explain the origins of human cultural prowess how is it that our ancestors began to diverge from other species? and the later cumulative elaboration of tools, technologies and conventions. We applaud O\&R for highlighting technical reasoning as an important piece of the puzzle, particularly with regards to the latter issue. However, their "technical reasoning hypothesis" cannot be seen as a competing theory to the more conventional emphasis on social learning, but rather as pinpointing one element in a feedback loop through which cognition, culture and technology co-evolve.

Acknowledgments. This work was supported by an ESRC grant (ES/ M006042/1) to AT, CAC and FH. The authors are grateful to Amanda Lucas for discussion.

Conflict of interest. None.

\section{What matters emotionally: The importance of pride for cumulative culture}

\section{Jessica L. Tracy (i)}

Department of Psychology, University of British Columbia, Vancouver, BC, Canada V6T $1 Z 4$.

jltracy@psych.ubc.ca

www.ubc-emotionlab.ca

\section{doi:10.1017/S0140525X20000072, e180}

\section{Abstract}

Osiurak and Reynaud highlight a major omission of models of cumulative technological culture. I propose an additional problematic omission: pride. By taking this emotion into account, we can address the question of why humans seek to learn, teach, and innovate - three processes essential to cumulative technological culture (CTC). By fostering achievement, prestige, and social learning, pride provides a pivotal piece of the puzzle.

Osiurak and Reynaud (O\&R) argue that cognitive factors have been overlooked in research on the emergence of cumulative technological culture (CTC). Here, I highlight another overlooked factor also critical to CTC. Although scholars agree that social learning and innovation are essential to cumulative culture, few have asked why humans engage in these behaviors, at a proximate level: what motivates people to teach others what they know, learn valuable knowledge and skills, and improve upon these with new innovations? The answer to all three questions, I argue, is pride, the self-conscious emotion that instills in humans a desire to develop and maintain a self-concept that meets and exceeds social norms and standards.

Pride is pleasurable emotion that reinforces socially valued behaviors (Tracy \& Robins 2004; Weidman et al. 2016). Numerous studies provide strong support for the universality of pride in humans (e.g., Tracy \& Matsumoto 2008; Tracy \& Robins 2008). At a proximate level, individuals' desire to experience pride motivates them to work toward effortful achievements and engage in moral behaviors; and their nonverbal displays of pride cross-culturally communicate to others their deservingness of high rank and prestige (Shariff \& Tracy 2009; Tracy et al. 2013; Witkower et al. 2019). From a genetic evolutionary perspective, then, it is likely that pride emerged in humans to solve the adaptive problem of social-rank attainment (Cheng et al. 2010). Yet, pride may have come to serve a secondary function in human history, facilitating three psychological processes that are critical to CTC: (1) a motivation to create, build, and discover; (2) a willingness to teach one's creations to others; and (3) an ability to selectively learn from others who are experts (Tracy 2016; Tracy et al., in press).

Beginning with that first critical process of developing skills and acquiring knowledge, pride is the emotion that pushes people to work effortfully in ways that ensure success; feeling pride increases persistence at boring tasks (Williams \& DeSteno 2008) and achievement-building activities that yield future rewards (Ho et al. 2016), and pride is associated with creativity and innovation (Damian \& Robins 2013). Furthermore, a felt absence of pride motivates under-performing individuals to change their ineffective work habits so as to become more productive and successful (Weidman et al. 2016).

Turning to the second critical process, a willingness to teach, here pride is crucial because it facilitates the attainment of prestige. Prestige evolved in humans as a rank attainment strategy distinct from dominance, the threat-based strategy shared widely across the animal kingdom. In humans, a species with the capacity for social learning, it becomes adaptive to defer to leaders who possesses valuable knowledge and skills (i.e., prestige), as doing so incentivizes these individuals to allow followers to copy and learn from them (Henrich \& Gil-White 2001). Pride is the affective mechanism underlying the attainment of prestige; in addition to motivating the achievement-orientation that results in knowledge and skill acquisition, pride motivates individuals to help and advise others, and to cooperate and demonstrate care for those in need (Ashton-James \& Tracy 2012; Cheng et al. 2010; Dorfman et al. 2014; Van Der Schalk et al. 2012). Further supporting this account, prestigious group members regularly feel the "authentic pride" that comes from achievements and prosocial behaviors but not the "hubristic pride" that is associated with aggression and dominance (Cheng et al. 2010), and display the pride nonverbal expression during rank contests, presumably to signal their prestige to potential followers (Witkower et al. 2019).

The third psychological process that underpins cumulative culture is strategic copying, in the sense of choosing to learn from the wisest or most skilled group members so that high-quality cultural knowledge is passed on. This process requires individuals to determine who is likely to be prestigious, then defer to those models and copy them (Henrich 2017). Studies show that even 2 -year-olds choose to learn from social models who demonstrate accurate, rather than inaccurate, knowledge (Koenig \& Woodward 2010), and, when lacking information about a model's accuracy, 
seek cues of expertise in the form of nonverbal displays of certainty (Birch et al. 2010; Brosseau-Liard \& Poulin-Dubois 2014). These displays are notably similar to the universally recognized pride expression (which includes smiling, expansive posture, and head-tilt upward; Tracy \& Robins 2008), pointing the potential importance of pride in social learning.

To test whether adults, too, selectively learn from social models who display pride, we incentivized participants to correctly answer a difficult trivia question and gave them the opportunity to copy an answer offered by a peer - who was actually a confederate, posing an expression of pride, shame, happiness, or neutral. When this individual displayed pride, participants copied them $80 \%$ of the time, compared to $50 \%$ for happiness, and $20-30 \%$ for shame and neutral expressions (Martens \& Tracy 2013).

Copying those who display pride is likely to be adaptive at a genetic level, by prompting learning that increases fitness; and also adaptive at a cultural level, by increasing the likelihood that the best knowledge and skills are passed on to others who can improve upon them further (Henrich 2017). Although people can fake pride displays, in the long run such performances are unlikely to shape social learning, a process that occurs largely in relationships formed across many different interactions, and which takes into account partners' long-term reputations. Indeed, even children eventually stop copying a social model who displays confidence but has a history of inaccuracy (Brousseau-Liard et al. 2014); and adults who are caught overclaiming what they know become distrusted and unattractive social partners (Tenney et al. 2019).

In sum, pride contributes to each of the three key psychological processes that underlie cumulative technological culture: it motivates people to work hard to achieve, thereby facilitating the development of valuable knowledge and skills; it promotes the attainment of prestige, which entails a built-in incentive to share the fruits of one's achievements with others; and it cues discriminatory social learning, informing group members which models they should copy and learn from.

Financial support. This work was supported by an Insight Grant from the Social Sciences and Humanities Research Council of Canada.

Conflict of interest. None.

\section{Causal learning in CTC: Adaptive and collaborative}

\section{Netanel Weinstein and Dare Baldwin (1)}

Department of Psychology, University of Oregon, Eugene, OR 97403.

netanelw@uoregon.edu baldwin@uoregon.edu

baldwinlab.uoregon.edu

doi:10.1017/S0140525X20000199, e181

\section{Abstract}

Osiurak and Reynaud highlight the critical role of technicalreasoning skills in the emergence of human cumulative technological culture (CTC), in contrast to previous accounts foregrounding social-reasoning skills as key to CTC. We question their analysis of the available evidence, yet for other reasons applaud the emphasis on causal understanding as central to the adaptive and collaborative dynamics of CTC.

Osiurak \& Reynaud (O\&R) detail a two-pronged case for technical-reasoning skills as primary in cumulative technological culture (CTC), looking to neurophysiological research on the one hand, and micro-society paradigms on the other. Our commentary will focus on the latter line of research.

In the micro-society paradigm, participants are tasked with building an artifact (e.g., a wire tower or paper airplane) within an allotted period. These artifacts (one for each participant) are then assessed on dimensions such as attractiveness, size, and functionality. Critically, participants complete the task as members of a "chain," such that upon completion of the task, the next participant in the chain completes the task as well. Participants either simply observe the actions of others in their chain, or are allowed to communicate with them before attempting to build the artifact on their own. Of interest are relationships that might emerge between various cognitive capacities and both individual and cumulative performance under these two conditions. O\&R's key conclusion from this research is that technicalreasoning skills predict cumulative performance better than social-reasoning skills.

In this research, technical-reasoning skills were assessed solely via participants' performance on two subsets of the Batterie Multifactorielle d'Aptitudes (NV7 battery), measuring ability to select appropriate tools for a given task and mental rotation. Similarly, social-reasoning skills were assessed via just two tasks: the Reading-the-Mind-in-the-Eyes Task (RMET), measuring ability to label emotions based on facial expressions, and the Comic Strip (CS) task, measuring ability to infer an agent's future action based on prior contextual information.

There are several reasons for questioning O\&R's findings as an adequate foundation for their inference that technical-reasoning skills account for CTC to a greater degree than social-reasoning skills. First, the only two measures O\&R employed to assess the role of social reasoning in CTC are unlikely to index individual variability in the types of social-reasoning skills that are argued to facilitate CTC (see Tomasello 2016). Specifically, the RMET indexes only the ability to accurately label a decontextualized facial emotional expression (see Aviezer et al. 2017); how this ability relates to general social understanding is not clear (see Oakley et al. 2016). As well, a recent meta-analysis of the psychometric properties of the CS revealed a negatively skewed pattern suggesting possible ceiling effects; it may not be well-suited for investigating individual differences (Davidson et al. 2018), and thus is likely inappropriate for assessing relationships between social reasoning and CTC.

Second, flawed logic affects O\&R's analysis: just because individual differences on particular social-reasoning tasks (e.g., RMET and CS) fail to predict outcomes (e.g., cumulative performance) doesn't mean those (or other) social-reasoning skills are not critical to the achievement of those outcomes. Key aspects of the skill at issue might be quite central to the outcome; aspects of the skill that happen to display individual differences may be what are irrelevant. To illustrate, consider a scenario in which researchers observe no correlation between performance 
on a particular working memory task and vocabulary size. It would be misguided to conclude from such a finding that working memory more generally is unrelated to language acquisition. Relatedly, a more general point: O\&R's case is problematic because it involves drawing a strong inference - social-reasoning skills aren't key to CTC - from a null finding (absence of a particular correlation).

Third, the micro-society paradigm, although innovative in its use of tangible dependent variables, does not adequately capture the cultural, historical, and ecological circumstances underlying CTC in the real world. Ironically, O\&R contrast their views with those of Boyd et al.'s (2011) "cultural niche" approach, yet Boyd and colleagues explicitly refer to adaptation (e.g., sophisticated hunting or shelter building methods) whereas the artifacts in O\&R's tasks (paper airplane and tower) do not serve what would normally be regarded as adaptive functions. Without genuine adaptive pressure, there may be little communal interest in achieving cumulative progress. Therefore, O\&R's micro-society findings may radically underestimate the role of social-reasoning factors in CTC.

Fourth, the micro-society paradigm is biased toward an individual-centered cognitivist approach because the constructed artifacts are always built by individuals who are alone in a room. Collaboration, an important component of the culturecentered approach to CTC (see Tomasello 2016), is thus not observable because of a task design that prohibits social interaction during the actual construction phase.

Finally, O\&R's conceptual analysis lacks precision. Specifically, they employ terms such as technical reasoning, mechanical knowledge, and causal understanding interchangeably to refer to the proposed primary "driver" of CTC, even though these terms are arguably distinguishable in meaning. Furthermore, the scope of O\&R's claim is not clear. In other places (e.g., De Oliveira et al. 2019), the authors limit their claims to technology to the exclusion of other forms of cumulative culture such as music (itself a distinction that is not self-evident), but such clarification is missing in the target article, generating genuine theoretical confusion. Finally, although O\&R describe theory-of-mind understanding as orthogonal to causal reasoning, it is important to note that causal reasoning is argued by many to be a defining feature of theory-of-mind development (e.g., see Penn et al. 2008 b). Pitting the two against one another is potentially misleading and theoretically problematic.

Similar to others (e.g., Gopnik \& Wellman 1994; Penn \& Povinelli 2007; Wellman \& Gelman 1992), we find it highly plausible that causal learning is a primary engine behind humans' acquisition of both sophisticated social- and technical-reasoning skills. Furthermore, despite the lack of evidence, we agree with $\mathrm{O} \& \mathrm{R}$ that causal understanding likely plays an important role in CTC with language - another inter-related skill-set - operating as a powerful catalyst (Sterelny 2016). Moving forward, we suggest that future research using the micro-society paradigm introduce both adaptational and collaborative components to achieve greater ecological validity and provide a more comprehensive view of the dynamics of CTC.

Financial support. This research received no specific grant from any funding agency, commercial, or not-for-profit sectors.

Conflict of interest. None.

\section{Refining our understanding of the "elephant in the room"}

\section{Andrew Whiten $\mathbb{1}$}

Centre for Social Learning and Cognitive Evolution, School of Psychology and Neuroscience, University of St Andrews, St Andrews KY16 9JP, UK. a.whiten@st-andrews.ac.uk

\section{doi:10.1017/S0140525X20000096, e182}

\section{Abstract}

The authors do the field of cultural evolution a service by exploring the role of non-social cognition in human cumulative technological culture, truly neglected in comparison with socio-cognitive abilities frequently assumed to be the primary drivers. Some specifics of their delineation of the critical factors are problematic, however. I highlight recent chimpanzee-human comparative findings that should help refine such analyses.

BBS peer-commentaries often favour criticism, including my own. Here I start instead with a broad note of approval. I judge Osiurak and Reynaud (O\&R) are essentially correct in arguing that pervasive claims for special forms of human social learning constituting the primary explanation for our distinctive cumulative technological cultures have neglected an "elephant in the room": non-social cognition. In an article cited by O\&R, Whiten et al. (2003) reviewed accumulating evidence of chimpanzees learning diverse kinds of object manipulation from others, implying similar capacities in our common cognitive ancestry. This led us to suggest that at the time ancestral hominins left evidence of early cumulative culture in the shape of knapped stone artefacts, "the really significant change ... was not so much in social learning mechanisms themselves, but rather in the kinds of behaviour patterns that were invented as increasing brain size made more sophisticated sequences of action possible. Existing capacities for imitation would be brought into play to acquire these from their inventors, for as encephalization made possible more complex innovations, so would it allow the imitator to represent more complex actions for copying" (p. 103).

O\&R do the field of cultural evolution a real service by elaborating on this last point, that sophisticated technological cognition may facilitate not only the innovations essential to cumulative culture, but also the representation of novel behaviours performed by others, such that they can be copied. However, not all of the authors' specific propositions are compelling. I highlight some limitations further below.

First, I highlight another observation made in Whiten et al. (2003) that supports the target article's core argument. This refers to hominin Acheulian stone tool making, which persisted with little cumulative change for over 1 million years. Experimental work on the acquisition of the skills necessary to knap Acheulian-type bifaces (e.g., Stout et al. 2019) indicates this requires at least imitative copying, if not some form of teaching. As Whiten et al. (2003) and earlier, Mithen (1996a) commented, this implies that such high-fidelity copying was not sufficient to create cumulative lithic culture; what was necessary was the 
invention of more sophisticated tool-making techniques, thence copied by others.

I wonder, however, why the authors of the target article are not more ambitious in addressing the elephant in the room beyond only cumulative technology? Human cumulative culture has spanned diverse domains, from language to rituals, religions, dance and greeting gestures, to name a few; doesn't the elaboration of these also require both faithful cultural transmission and bouts of innovative non-social cognition?

With this in mind I'd question the authors' reference to "reason" as critical to the non-social cognitive component - does one need to "reason" to innovate a new dance step or a novel ritual flourish? Even considering the authors' insistence (sect. 2.1) that "technical reasoning is critical for the learning of technical content from social interactions as well as for improving this content", numerous empirical findings appear in conflict, including the claimed unique manifestations in humans. For example in the original finding nowadays referred to as over-imitation, Horner and Whiten (2005) discovered that young chimpanzees omitted visibly unnecessary elements of a tool use demonstration when their turn came, thus apparently displaying more "technical reasoning" than children, who blanket-copied both necessary and causally unnecessary elements. Such findings seem at odds with the authors' assertion 'that technical-reasoning skills allow humans to extract relevant technical information from a 'social demonstration' (imitation), [and] to reject irrelevant information" - in our experiment, the latter is what the chimpanzees did, but the children did not.

This is but one instance in which I suggest more work is needed to more accurately delineate the nature of the non-social cognition that the authors urge us to focus on to better understand cumulative culture. Below there is space to list just a few more, with a focus on human/chimpanzee cognitive comparisons.

In Section 2.1, it is said that humans are the only species to display cognitive transfer skills. What then of cases such as that reported by Tennie et al. (2010) in which chimpanzees were shown by a human that pouring water from a bottle could lift a peanut in a cylinder, to retrieve it? Later, presented with this problem but no bottle, several chimpanzees transferred their knowledge of getting water from their drinker and spitting it, to instead fill the cylinder by this means: a real and imaginative innovation on their part, achieved by this transfer. In reviewing animal tool use, Visalberghi et al. (2017) cite over 60 papers in their section on "transfer."

It is nevertheless true that chimpanzees often fail to achieve cumulative social learning in contexts where humans do. In the first demonstration of this, young chimpanzees learned a simple technique of using a probe to extract honey from an artificial foraging device, but then failed to adopt a more complex and profitable demonstrated technology, which other chimpanzees who had not learned the simple technique did discover (Marshall-Pescini \& Whiten 2008). The first group thus seemed to get "stuck" on the simple technique, whereas children later tested in a similar context did "step up" and adopt the more sophisticated and productive option after they witnessed it (Whiten et al. 2009). More recent experiments have however revealed some instances of cumulative technology through chimpanzees' combination of discoveries made by others (Vale et al. 2017), and where cognitive loads involved in stepping up to a more complex technology are reduced (Davis et al. 2016; 2018).

The authors have made a valuable contribution to the field of cultural evolution, but more refined, and sometimes more nuanced, understanding of the aspects of non-social cognition key to cumulative culture are called for. Comparative studies focused on cumulative culture issues such as those cited above are clearly relevant, but so may be new syntheses and thinking on such core-related topics as problem solving (Seed \& Mayer 2017), innovation (Kuczaj 2017) and causal reasoning (Völter \& Call 2017).

Financial support. This research received no specific grant from any funding agency, commercial or not-for-profit sectors.

Conflict of interest. None.

\section{Authors' Response}

\section{The elephant in the China shop: When technical reasoning meets cumulative technological culture}

\author{
François Osiurak ${ }^{\mathrm{a}, \mathrm{b}}$ ๑ and Emanuelle Reynaud ${ }^{\mathrm{a}}$ \\ ${ }^{\mathrm{a}}$ Department of Psychology, University of Lyon, 69007 Lyon, France and ${ }^{\mathrm{b}}$ French \\ University Institute, 75231 Paris, France. \\ francois.osiurak@univ-lyon2.fr https://emc.univ-lyon2.fr/fr/equipes/equipe- \\ cognition-outils-systemes/francois-osiurak/francois-osiurak-610797.kjsp \\ emanuelle.reynaud@univ-lyon2.fr https://emc.univ-lyon2.fr/fr/equipes/ \\ equipe-cognition-outils-systemes/emanuelle-reynaud/
}

doi:10.1017/S0140525X20000291, e183

\begin{abstract}
The commentaries have both revealed the implications of and challenged our approach. In this response, we reply to these concerns, discuss why the technical-reasoning hypothesis does not minimize the role of social-learning mechanisms - nor assume that technical-reasoning skills make individuals omniscient technically - and make suggestions for overcoming the classical opposition between the cultural versus cognitive niche hypothesis of cumulative technological culture.
\end{abstract}

\section{R1. Introduction}

Cumulative technological culture (CTC) is a fascinating phenomenon, considered in 2005 by the journal Science as one of the 125 big scientific questions. Research on this topic has been dominated by disciplines such as anthropology, evolutionary biology, mathematics, or archeology. Some areas of psychology also contribute to the debate (e.g., developmental and comparative). However, this rarely applies to the areas of psychology involved in the cognitive sciences, such as cognitive psychology, neuropsychology, or cognitive neuroscience (Heyes 2018). This is surprising if we acknowledge that CTC cannot be fully understood without a detailed examination of its neurocognitive origins. In the target article, we offered a unified cognitive approach to this phenomenon to overcome this shortcoming.

Before we begin our response by reminding the reader of the key aspects of our approach, we warmly thank the authors of all 26 commentaries for their thoughtful critiques and the extensions they suggested to the target article. Because of the great number of 
commentaries, we are unfortunately not able to discuss all of the concerns raised but hope we have done justice to the most important points.

\section{R2. What the elephant says}

The key hypothesis of our approach is that CTC originates in nonsocial cognitive skills, namely technical-reasoning skills which enable humans to acquire new techniques and improve existing ones. This hypothesis leads us to decouple CTC from sociallearning mechanisms without, however, wishing to minimize the impact of the latter (Gruber). Technical reasoning is a cognitive structure which makes it possible to acquire and elaborate technical content (i.e., mechanical knowledge). It is unaware of the source that provides this content. As rightly emphasized by Gabora and Smith, the distinction commonly made between asocial learning and social learning does not exist for technical reasoning. To paraphrase them, we can acquire the technique of peeling by watching a conspecific peel a banana (social learning), by watching a monkey peel a banana (asocial learning), or by watching the petals of a tulip unfold (asocial learning). In all these situations, the same technical reasoning is at work. What differs, however, is the opportunity offered by the environment. In other words, humans can acquire technical content more quickly through social learning - and particularly through complex forms of social learning such as teaching - than through asocial learning. Nevertheless, the opportunity to acquire technical content (through asocial or social learning) is orthogonal to the ability to acquire and improve it (through technical reasoning). As rightly pointed out by Claidière, our approach is far more consistent with a social than an ecological view of CTC.

This approach can be misinterpreted as placing exaggerated emphasis on the technical dimension of the phenomenon (e.g., Houkes \& Vaesen; Miu, Boyd, Richerson, \& Morgan [Miu et al.]; Thornton, Happé, \& Caldwell [Thornton et al.]). For instance, it might be considered to lead, by extrapolation, to the idea that "a single human, of infinite lifespan, with infinite time, would be able to produce the entirety of the current human repertoire" (Miu et al.). We understand how such a misinterpretation can be arrived at and we think that the main reason is that we did not specify what we meant by "the opportunity offered by the environment." We would like to correct this omission. Let us consider a solitary species equipped with technicalreasoning skills (Asocial opportunity+; Social opportunity-). This species can acquire technical content through asocial learning (i.e., innovation-by-invention; Rawlings \& Legare). However, the lack of opportunity to watch other conspecifics should prevent this species from showing the signs of CTC, with each individual being systematically condemned to reinvent the wheel. Therefore, some social conditions such as gregariousness and social tolerance are prerequisites for the social transmission of technical skills (van Schaik et al. 1999). ${ }^{1}$ Let us now consider a species with such social conditions (Asocial opportunity+; Social opportunity+). Even with less complex forms of social learning (i.e., no teaching), the ability to reason technically about the opportunities offered by the asocial and social environments can lead this species to progressively show the signs of CTC, thereby generating a greater number of innovations and, notably, innovations-by-modification (Rawlings \& Legare). However, the scope of this phenomenon may be limited because of the complexification of technology over time (see sects. 3.3 and 3.4). Thus, more complex forms of social learning based on specific social cognitive skills (i.e., theory of mind; but also language, an aspect overlooked in the target article; see sect. 5; Claidière; Thornton et al.) can become necessary to help boost CTC (Asocial opportunity+; Social opportunity $++\cdots+$ ). Having clarified these various issues, we can now address the major concerns raised by the commentators.

\section{R3. Is the elephant novel?}

Whiten judged that our approach was "essentially correct in arguing that pervasive claims for special forms of human social learning constitute the primary explanation for our distinctive cumulative technological cultures have neglected an 'elephant in the room': non-social cognition" (p. 46). As stated, the strength of our approach lies in the epistemological shift we proposed, stressing that CTC is not only a social but also a technical phenomenon. This shift has been influenced by our own scientific background, namely cognitive science, which provides novel insights into the neurocognitive origins of CTC. As discussed in sect. 2.1 (see also Fig. 2; Osiurak et al. 2020b), neuropsychological findings have demonstrated that technical reasoning supports both the use of familiar tools (e.g., using a hammer with a nail) and the use and making of novel tools to solve mechanical problems. Technical reasoning mainly involves the area PF within the left inferior parietal lobe. Interestingly, this brain area is also preferentially activated when people observe tool-use actions performed by others. In other words, this suggests that the same neurocognitive process (i.e., technical reasoning/left area $\mathrm{PF}$ ) is involved when humans carry out mechanical actions as well as when they watch others doing so (Reynaud et al. 2019). These findings are not anecdotal in a field where it is common to assume that "imitation/social learning" and "innovation/asocial learning" are two orthogonal dimensions of CTC (e.g., Miu et al.), and which therefore risks giving rise to only a very restricted account of cognition (Gärdenfors, Högberg, \& Lombard [Gärdenfors et al.]). Instead, in line with Gabora and Smith's commentary (see sect. R2), these neuroscientific findings indicate that the same cognitive process is at work whatever the nature of the learning (i.e., asocial and social). In this respect, contrary to what Miu et al. claim, the cognitive approach we propose offers a fresh look at CTC, as pointed out by Whiten and other commentators (Benge \& Scullin; Gärdenfors et al.; Gruber; Haber \& Corriveau; Korth; Motes-Rodrigo, Reindl, \& Bandini [Motes-Rodrigo et al.]; O'Brien \& Bentley; Rawlings \& Legare; Rutz \& Hunt; Thornton et al.; Tracy).

Another point that leads us to think that our approach is novel concerns the clarifications provided by some proponents of the cultural niche hypothesis about the role of technical reasoning, an aspect ignored so far (see immediately above). More specifically, Derex and Boyd state that "Boyd et al. (2011) and Derex et al. (2019) never claimed that reasoning plays no role in CC. Rather they argued that the improvement of culturally evolving technology is not necessarily tied to individuals' level of understanding" (p. 23). This statement corresponds to a considerable revision of the initial cultural niche hypothesis and we look forward to reading a future theoretical development of this hypothesis explaining what the precise role of technical reasoning in CTC is and how the cultural niche hypothesis now differs from the cognitive niche hypothesis. We also appreciate the clarification that the authors provided concerning the link between individuals' level of understanding and cumulative improvement. We have to note that this differs from the conclusions drawn by Derex et al. (2019): “These results indicate that complex technologies 
need not result from enhanced causal reasoning but, instead, can emerge from the accumulation of improvements made across generations" (p. 446). Miu et al. argue that we cited only a single (self-authored) study to support our link between technical reasoning and CTC. Actually, we cited four of our studies (De Oliveira et al. 2019; Osiurak et al. 2016; 2020a; in press). We acknowledge that this remains insufficient. Nonetheless, we have already collected the data from more than 1,200 participants who took part in micro-society experiments (some of these data are still unpublished). In all these studies, the conclusion is always the same: Cumulative performance is tied to individuals' level of technical reasoning. In broad terms, these findings invalidate the above prediction made by Derex and Boyd. Having said this, we agree with Derex and Boyd that "a more fertile approach may be to study how both types of abilities reinforce each other" (p. 24). Indeed, our target article was an attempt in this direction in that our aim was to develop a theoretical framework detailing how technical reasoning can work in concert with social-learning mechanisms to favor the emergence of CTC. Miu et al. hold that our approach is not sufficient because it does not explain "how individual-level decisions scale up to population-level dynamics" (p. 34). We agree and we also acknowledge that proponents of the cultural niche hypothesis have developed fruitful computational models of CTC at a macroscopic level. One of our current objectives is to follow this direction by conducting modeling work based on the technical-reasoning hypothesis of CTC. However, to initiate this fertile approach, proponents of the cultural niche hypothesis have also to integrate the key neuroscientific findings reported above as well as the link we identified between each individual's level of technical reasoning and cumulative performance in micro-society experiments.

\section{R4. Is the elephant solitary?}

As discussed in sect. R2, the focus placed in the target article on the technical dimension seems to have been misinterpreted or overinterpreted by several commentators. As O'Brien and Bentley highlight: "We would argue that this revised assumption in no way allows us to ignore the social dimension and redirect our focus to the nonsocial, strictly technical dimension" (p. 37). We fully agree with them. As explained, technical reasoning is the cognitive structure allowing individuals to acquire and improve technical content. However, the opportunity to acquire it increases as social-learning mechanisms become more effective (e.g., teaching). Thus, we did not hypothesize that solitary individuals equipped with technical reasoning would exhibit CTC. We also thank Derex and Boyd for stressing that "individuals constantly face intense tradeoffs and so must allocate their time and energy strategically" (p. 24). We did not address this point explicitly in the target article, although we agree that social-learning mechanisms (particularly teaching) provide suitable conditions for such strategies ${ }^{2}$ (e.g., deciding to acquire a new technical content through social learning when the cost of asocial learning is high). In broad terms, as pointed out by Gruber, our purpose was not to minimize the impact of social learning in CTC, but to shift the focus to the technical dimension by assuming that this phenomenon could not emerge without technical reasoning. More specifically, an aspect of our approach that seems to have been mis/overinterpreted by several commentators is the role of teaching/social demonstration in CTC (e.g., Derex \& Boyd; Gärdenfors et al.; Houkes \& Vaesen; Miu et al.; Moll, Nichols, \& Pueschel [Moll et al.]; Rawlings \&
Legare; Thornton et al.). These commentaries mention several studies in which young children fail to solve tasks by themselves (e.g., using water as a tool, or making a hook), but succeed after social demonstration (Beck et al. 2011; Moll 2018; Nielsen 2013). For some of the aforementioned commentators, this demonstrates that teaching/social demonstration is enough for subjects to acquire technical content, without any need to add technical reasoning to the equation. Here, we explain why our approach better fits these findings.

Let us begin with the exclusive hypothesis that teaching/social demonstration is enough to permit the acquisition of technical content. This hypothesis predicts that when a child or an adult is unable to acquire a technique by herself or himself, she or he should succeed after teaching/social demonstration. The study of Cutting et al. (2011) provides data that are useful for testing this prediction. They reported that 8 and $30 \%$ of the 4-5-year-olds and 6-7-year-olds, respectively, were able to make a hook by themselves to reach a target, whereas the values were 79 and $67 \%$ after a social demonstration (Experiment 1). The authors also proposed another task consisting of unbending a pipe cleaner to reach a target. In this task, 33 and $56 \%$ of the 4-5-year-olds and 6-7-year-olds, respectively, successfully solved this task, whereas 58 and $41 \%$ did so after a social demonstration (Experiment 1). These findings indicate that the effect of teaching/social demonstration is far from systematic because a significant number of children did not benefit from social demonstration. In broad terms, these findings invalidate the prediction derived from the exclusive hypothesis of teaching/social demonstration.

It is noteworthy that the discussion initiated in these commentaries is based on the incorrect assumption that technical reasoning alone (i.e., without social learning) can allow individuals to acquire spontaneously any technical content. This mis/overinterpretation corresponds to an omniscient view of technical reasoning (see sect. 2.3). However, we did not hypothesize that technical reasoning is a "magic bullet" (Miu et al.; Thornton et al.) making it possible to spontaneously and quickly understand our physical world or acquire and improve techniques. As detailed in sect. 2.3, individuals acquire techniques (i.e., mechanical knowledge) progressively over time and the opportunities offered by the environment (mostly social) differ between individuals, so that the degree of technical expertise is subject to considerable interindividual variation. The corollary is that not every individual can learn any given technical content at any given time. This idea is perfectly in line with the concept of zone of proximal development (Vygotsky 1978; see sect. 3.5; see also Haber \& Corriveau). In this frame, the aforementioned developmental studies suggest that teaching/ social demonstration can be, under some circumstances, a very effective way for children - although this is also true for adults to go just beyond their zone of proximal development. However, if what is transmitted socially goes far beyond this area, the acquisition of a new technical content becomes impossible. In this respect, this explains why some children can benefit from social demonstration although others do not, namely because this acquisition depends on their current level of technical-reasoning skills and, as a result, zone of proximal development.

In other words, the amount of technical information that can be transmitted during a social interaction depends very greatly on the learner's technical-reasoning skills, as demonstrated in several of our micro-society experiments (e.g., Osiurak et al. 2016). This interpretation is perfectly consistent with Haber and Corriveau, who argue "for a more integrated approach to cumulative 
technical culture, grounded in sociocultural theory (e.g., Vygotsky 1978). Such an approach should highlight the important cognitive skills children bring to bear to acquire technical information, yet also recognize that most learning situations occur in interactions with others" (p. 28). Some of the mis/overinterpretations discussed here might have been avoided if we had written this statement as eloquently as Haber and Corriveau have done. In light of this discussion, we must fine-tune our suggestions concerning the idea that children might not have the technical expertise necessary for them to exhibit CTC (sect. 3.4). We thank Burdett and Ronfard for highlighting this concern. Studying social transmission in children can, of course, be a good methodological choice for exploring CTC. Simply, in light of the present discussion, we meant that there can be no doubt that much more care is needed when designing experimental tasks in which the technical solutions fall within the participants' zone of proximal development. This might optimize the chances of observing cumulative performance.

\section{R5. Is the elephant omniscient?}

In the target article, we formulated two assumptions that may appear paradoxical, but are not. The first is that technical reasoning enables humans to acquire and generate a great amount of technical content. The second is that individuals acquire techniques (i.e., mechanical knowledge) progressively over time depending on the opportunities offered by the environment, leading to considerable interindividual variations. More specifically, we meant that technical reasoning offers the technical potential to acquire any technique. Nevertheless, these techniques cannot be learned independently of a certain logic of acquisition (i.e., the zone of proximal development discussed above), as nicely illustrated by the work of Baillargeon and colleagues (see sect. 2.3). Thus, humans are not omniscient in terms of techniques and their acquisition is costly in terms of time. In addition, given that humans have developed a vast amount of techniques in distinct technical domains, it is, of course, impossible for a human to acquire all of them during her or his lifetime (i.e., the core principle of CTC). The corollary is that, one day, the techniques of a single domain might be too complex to be acquired and improved in a single lifetime (Mesoudi 2011). Regardless, assuming that each human possesses the technical potential to acquire a great - perhaps infinite - amount of technical content is not inconsistent with the idea that our lifetimes, along with the very complex form taken by CTC, do not allow humans to master every technique of every domain. The corollary is the growing specialization of the domains of expertise of individuals. $^{3}$

Following on in this vein, it is conceivable that technical expertise in some domains can be lost because of epidemics that primarily affect the experts involved in these domains. The problem is that the surviving individuals might have developed very little expertise in these domains - perhaps because of their idiosyncratic historical trajectories, which have led them to become experts in other (not necessarily technical) domains. The consequence is that these surviving individuals may be unable to re-develop the lost techniques because their acquisition/discovery falls far beyond the survivors' zone of proximal development in the corresponding domain. The technical potential of these survivors can nevertheless enable them to re-master the lost techniques if experts from the technical domain in question visit and help them to reincorporate these techniques. This example corresponds to the Polar Inuit example provided by Miu et al. as a counterargument to our approach. However, as explained here, the technical-reasoning hypothesis of CTC offers an interesting alternative for explaining such phenomena, and one which anchored in a more cognitive and developmental framework.

\section{R6. What the elephant brain tells us about СТC}

As stated in section R3, one of the strengths of our approach is to include a consideration of the literature on cognitive science in our attempt to understand the origins of CTC. This integrative approach also appears to be fruitful to address some concerns raised in several commentaries. Let us start with the question of why we did not use the more generic term "causal cognition/ understanding" instead of technical reasoning (Gabora \& Smith; Gärdenfors et al.; Weinstein \& Baldwin). This distinction was initially made on the grounds that technical reasoning is thought to be not only causal but also analogical (sect. 2.1). As stressed, even if some nonhuman animals - including tool-using species - may exhibit signs of causal understanding, evidence is still needed concerning the analogical component (for a similar view, see Rutz \& Hunt; Taylor \& Jelbert). In other words, the terms causal cognition/understanding and technical reasoning cannot be used interchangeably. Gabora and Smith and Gärdenfors et al. also argue that causal cognition refers to a domain-general ability allowing humans to reason not only about the physical world but also about the social world. In other words, the emergence of this ability could have led to the joint development of human technicalreasoning and theory-of-mind skills. We agree that theory-of-mind skills are fundamental for inferring how our conspecifics may behave based on our knowledge of their guessed hidden mental states. This is a form of causal reasoning: "I think that Emily has put on makeup because she wants to be beautiful for the prom." Nevertheless, the fact that technical reasoning and theory of mind are two forms of causal reasoning does not mean that they are supported by the same neurocognitive processes. The former is based on knowledge about the mechanical actions that can be performed in the physical world. The latter is based on knowledge about the hidden mental states that humans can possess. This distinction is well illustrated by Crespi, who discussed the difference between mechanistic cognition and mentalistic cognition on the basis of the literature on the autism spectrum. A large body of evidence also comes from the neuroscientific literature, which has repeatedly confirmed that technical-reasoning and theory-of-mind skills involve distinct neural networks: the area PF within the left inferior parietal lobe (see sect. 2.1) and the dorsomedial prefrontal cortex, temporoparietal junction, and anterior temporal cortex, respectively (e.g., see Van Overwalle \& Baetens 2009; see also Spunt \& Lieberman 2012 for a distinction between the two networks in the same study). Technical reasoning is impaired in patients with apraxia of tool use (Osiurak \& Rossetti 2017), whereas theory-of-mind disorders are commonly observed in most psychiatric disorders. It is therefore not viable for a neuroscientist to consider that technical reasoning and theory of mind are two manifestations of the same ability.

Another point concerns our discussion on reverse engineering. We focused on the case where an individual knows the function of a tool and has to reason about how it can be used or built (pure reverse engineering). Hernik suggests that we might be "right in pointing to the role of naïve mechanical principles in these processes" (p. 29). However, as Hernik stresses, we did not elaborate 
on the opposite case, that is, where an individual has to infer the conventional function of an artifact (e.g., a hammer) she or he has never seen before (hereafter called teleofunctional inference). As a matter of fact, we have already discussed this aspect and corroborated Hernik's hypothesis that humans might solve teleofunctional inference situations by using a kind of "teleological reasoning" (see Osiurak 2017; Osiurak \& Badets 2016; Osiurak et al. 2008). In the case of reverse engineering, individuals know the function (i.e., the goal) of the tool in question. The task is to infer the potential technical means allowing them to use or make the tool. This task is very close to novel tool-use tasks in which participants have to find out how to use or make novel tools to solve mechanical problems (see sect. 2.1). It is not possible to consider that familiar tool-use tasks (e.g., using a hammer with a nail) correspond to reverse engineering because the participants already know the tools. This is why this task is called familiar. Nevertheless, familiar tool-use tasks can become novel tool-use tasks if the individual does not know the tools. This is the case of patients with semantic disorders (e.g., semantic dementia) after damage to the anterior temporal cortex. These patients can be unable to name or describe the conventional use of familiar tools. Interestingly, these patients perform relatively well in novel tool-use tasks, as well as in familiar tool-use tasks, when both the tool and the object are presented (e.g., Baumard et al. 2016; Hodges et al. 2000; Lesourd et al. 2016; Osiurak et al. 2008; Silveri \& Ciccarelli 2009). These findings contrast with those obtained in patients with lesions to the left inferior parietal lobe (i.e., the neural basis of technical reasoning), who can be severely impaired on both familiar and novel tool-use tasks (see sect. 2.1). In sum, these findings demonstrate that technical reasoning is critical to reverse engineering.

The question is how these patients with semantic disorders behave when they are presented with a single familiar tool (i.e., teleofunctional inference). In this case, the evidence indicates that they experience great difficulties in demonstrating the conventional use (see Hodges et al. 2000; Osiurak et al. 2008; Sirigu et al. 1991). Nevertheless, their behavior still seems to be driven by their spared technical-reasoning skills, leading them, for instance, to use a screwdriver as a gimlet (see Osiurak et al. 2008). In other words, the degradation of knowledge about the social usages of familiar tools and objects prevents them from performing the "teleological reasoning" highlighted by Hernik, but not from making use of technical reasoning to find a potential use for the tool, and this search for technical means remains a necessary component of teleofunctional inference. As mentioned above, these patients have lesions to the anterior temporal cortex, a brain area involved in the neural network involved in theory of mind (see above). This explains why they can present theory-of-mind impairments (Duval et al. 2012; Irish et al. 2014). Taken together, these findings extend the discussion initiated by Hernik, by suggesting that teleological reasoning might be specifically involved in teleofunctional inference but not in reverse engineering. However, these findings also indicate that technical reasoning remains necessary in both. Therefore, this leads us to assume, contrary to Hernik, that the structure underpinning technical reasoning is critical for the progressive emergence of CTC, even if teleological reasoning (probably based on theory-of-mind skills/functional knowledge) might have helped boost it later during evolution.

Moll et al. also address the question of the role of technical reasoning in teleofunctional inference situations. They reported a small study they conducted, in which they presented participants with an olive/cherry pitter (an artifact also used by Humphreys
2001 to elaborate on his theory of affordances). This is a very unfamiliar tool which is useful for removing stones. They found that only one of the 21 adults they met was able to find the correct conventional use of this tool. For Moll et al., this demonstrates the clear limits of the technical-reasoning hypothesis of CTC and particularly the link we draw between technical reasoning and reverse engineering. Again, as stressed above, this statement seems to be anchored in an omniscient view of technical reasoning which we do not support. However, as rightly stressed by Hernik, this is not a proper case of reverse engineering, but rather of teleofunctional inference. Consequently, as discussed immediately above, technical reasoning is not sufficient to infer the conventional use of a tool that an individual has never seen before. Instead, if Moll et al. wished to conduct another small study, they could present their participants with a real reverse-engineering condition by asking them how to use this tool with an olive or a cherry, or simply by proposing both the tool and the olive/cherry. In this case, we predict that most of the participants would be able to find the solution.

Motes-Rodrigo et al. hold that we "might be wrong in assuming that [working memory] only allows for storing mechanical actions... [working memory] could allow for combinations of mechanical actions - there might be no need to assume separate technical reasoning skills" (p. 36; for a similar point, see Gabora \& Smith). Working memory is commonly defined as a system devoted to allocating a limited pool of attentional resources to the simultaneous maintenance and processing of information (Barrouillet et al. 2011; Plancher \& Barrouillet 2020). In line with this, we agree with Motes-Rodrigo et al. that working memory provides the attentional resources needed for the manipulation of mental representations in the short term. However, contrary to what they argue, working memory does not, strictly speaking, manipulate/process these mental representations. Let us illustrate this point with an example from the technical domain, namely the making of a simple spear. If an individual intends to plan the full sequence of mechanical actions required to make a simple spear, she or he will face the challenge of solving a series of technical problems. For instance, Problem A is to make something pointed enough to go through the prey. Solution A can be to make a pointed stone. Problem $B$ is to transform a stone into a pointed stone. Solution B is to use a hammerstone, and so on. Note that the generation of some solutions can place constraints on the solutions produced subsequently, or even create new problems (e.g., the shape and size of the shaft of the spear have to be adapted to the point to connect them together). As detailed in sect. 2.4, each of these problems is solved through technical reasoning. Working memory is useful here for allocating attentional resources to the maintenance and processing of information (e.g., maintaining Solution A although solving/processing Problem B). The allocation of these resources can quickly become a complex task if the sequence increases and if the different technical problems are interconnected (e.g., solving/processing Problem D, which is based on the maintenance of Solutions A, B, and C). This example clearly illustrates that the role of working memory is not to "manipulate" or "process" information, strictly speaking, but rather to allow other cognitive processes to manipulate information that does not yet exist in the environment. The corollary is that technical reasoning and working memory need to be understood separately because they are based on distinct neurocognitive processes, contrary to what Motes-Rodrigo et al. suggest. A significant body of neuropsychological evidence has confirmed that these two cognitive abilities can be impaired independently (for a review, see Osiurak 2014). 
As explained in sect. 2.1, our approach is also in line with longterm working-memory models (for a similar view, Wynn \& Coolidge 2014), which are very instructive for understanding how expertise can interact with working memory. The challenge for working memory is to allocate attentional resources to both the maintenance and processing of information. In this frame, if less resources are devoted to processing information, then more resources can be assigned to the maintenance of information. Experts are characterized by their ability to process information more quickly in their domain of expertise. The corollary is that an expert, say, in a specific technical domain (e.g., weaponmaking) will be able to solve each of the technical problems raised by the making of a simple spear more quickly. Therefore, she or he will have more attentional resources available to maintain the different technical solutions generated and, as a result, be able to progress further in the task and undertake full planning. By contrast, the cost of processing will be higher for a non-expert, leading her or him to have less attentional resources available for maintenance. The consequence is that she or he will have to engage in partial planning, by progressing in the task based on environmental feedback and trial and error. This is an instance of reasoned trial and error, with behavior being driven by a number of technical solutions generated through technical reasoning (but not all the technical solutions required to complete the task). Gabora and Smith stressed that, strictly speaking, trial and error cannot be reasoned. We hope that this clarification will make it easier to understand that trial and error can also be reasoned (see also Vaesen 2012). More importantly, they also claim that our approach to "working memory contradicts decades of research on the psychology of creativity" (p. 25). We partly disagree with this critique. The interaction of expertise with working memory as described above implies that an expert in a specific domain will be able to manipulate a greater number of items of information in the short term and, thus, generate more suitable and novel ideas than a non-expert. This conception of creativity is perfectly consistent with some studies on creativity that has demonstrated that the degree of expertise (i.e., the amount of knowledge) in a specific domain is an important prerequisite for creativity (e.g., Simonton 2000; for further discussion on this aspect, see also footnote 7). In other words, our approach to working memory does not contradict the whole body of psychological literature on creativity.

\section{R7. On the origins of the elephant}

The culture-gene coevolutionary approach assumes that culture has shaped the human genome by driving the evolution of both our bodies and brains (e.g., Laland et al. 2010). In this approach, the focus has mainly been on the adaptation of social cognitive skills (e.g., see Chudek \& Henrich 2011), as also suggested by the cultural niche hypothesis (Boyd et al. 2011), which is akin to this approach. Our approach is consistent with this, although it differs by suggesting that culture (but not CTC) could also have favored the emergence of non-social cognitive skills, such as technical reasoning. We would like to elaborate on this point and this will also give us the opportunity to discuss the idea that our "account is evolutionarily impossible, as it assumes that ancestral hominins must have made a giant cognitive leap to evolve modern technical reasoning skills before cumulative culture could get off the ground. O\&R provide no suggestions as to what selective pressures might drive the evolution of technical reasoning in ancestral populations" (Thornton et al.; p. 43).
First of all, we do not consider that culture or any other form of cultural evolution originates in technical reasoning (for a similar viewpoint, see Claidière). The technical-reasoning hypothesis of CTC is consistent with the assumption that some specific social conditions (i.e., gregariousness and social tolerance) are the prerequisites for the emergence of social learning and, a fortiori, culture (see sect. R2). It is precisely in such a context that technical reasoning, in concert with "simple" forms of social learning, can lead to signs of CTC. This does not imply that all the cognitive components of technical reasoning have evolved spontaneously and that some of them are not shared with other species. Evidence clearly indicates that nonhuman animals can show causal understanding of the physical world (see sect. 2.1). Some specific components, such as the analogical component, could have nevertheless emerged in our human ancestors, making technical reasoning unique to our lineage (i.e., no giant cognitive leap as claimed by Thornton et al.). The reason for this emergence does not concern survival, strictly speaking (e.g., see Toth 1985). Instead, these components could have been selected because they conferred an adaptive advantage in terms of food acquisition or protection, putting pressure on the other species (a proposal very similar to the cognitive niche hypothesis). ${ }^{4}$ Paleoneurological studies could be carried out to test whether our human ancestors possessed the parietal structure underlying technical reasoning (i.e., the area PF within the left inferior parietal lobe), as suggested by Gruber. In addition, our approach posits that the development of CTC could have generated a technology-rich environment made up of more and more complex and "opaque" tools, ${ }^{5}$ which could have favored - among other things (see Claidière) - the selection of specific social cognitive skills (i.e., theory of mind) which are useful for increasing social transmission. On this point, the technical-reasoning hypothesis of CTC is in line with the culture-gene approach and the cultural niche hypothesis. Indeed, it also provides a good way to reconcile the cognitive niche and the cultural niche hypothesis: Culture $\rightarrow$ Non-social cognitive skills (technical reasoning) $\rightarrow$ Signs of $\mathrm{CTC} \rightarrow$ Social cognitive skills (theory of mind) $\rightarrow$ Complex forms of CTC.

Taylor and Jelbert and Rutz and Hunt emphasize that the signs of CTC can also be observed in New Caledonian crows, a species known for its causal-understanding skills. A recent study also reported that New Caledonian crows can improve their tool-making behavior by means of situations very close to reverse engineering (Jelbert et al. 2018). However, evidence also shows that they can benefit from "simple" but not from more complex forms of social learning (Logan et al. 2016). In broad terms, as stressed by these commentators, these findings confirm the idea that signs of CTC can emerge from the interaction between non-social cognitive skills and "simple" forms of social learning, partly corroborating our approach. We say "partly" because they also challenge this. Indeed, one of our key assumptions is that signs of CTC should be observed only in species equipped with technical-reasoning skills, particularly humans. Even if we can elaborate on the fact that New Caledonian crows might not possess all the cognitive components of technical reasoning (notably the analogical component), it remains that these findings imply that CTC may occur even with less elaborate forms of "physical understanding." Therefore, resolving this discrepancy should lead to some fascinating future research dedicated to exploring this aspect. In the meantime, we have to acknowledge that these findings do not validate some of our predictions. 


\section{R8. Is the elephant too micro?}

In the target article, we discussed in detail - perhaps too much detail - the results obtained from micro-society experiments and particularly those that used reverse-engineering conditions. We also mentioned that experiments of this kind suffer from the limitation that they do not reflect how technical transmission occurs in everyday life (sect. 5), a limitation also stressed by O'Brien \& Bentley. We acknowledge that this could have created another mis/overinterpretation of our approach, consisting of thinking that human ancestors could have shown signs of CTC on the basis of reverse engineering alone (for related concerns, see Derex \& Boyd; Thornton et al.). In fact, the technicalreasoning hypothesis of CTC assumes that human ancestors could have developed CTC on the basis of reverse engineering and other "simple" forms of social learning (e.g., observation). Nevertheless, reverse-engineering conditions are very instructive because they allow us to test without ambiguity whether CTC can emerge without complex social learning. We hope that this clarification will help to correct this mis/overinterpretation.

The methodology employed in all our published studies using micro-society paradigms consisted of performing additional testing to assess certain participants' cognitive skills to link them to the performance reported in the chains (e.g., Osiurak et al. 2016). This methodology was informative in indicating that learners' technical-reasoning skills are systematically the better predictor of cumulative performance, a key finding for our approach. We also assessed theory-of-mind skills by using two tasks, namely, the Comic Strip task and the Reading-the-Mind-in-the Eyes task. Weinstein and Baldwin argue that this methodological choice can explain why we did not find any link between theory-of-mind skills and cumulative performance in our microsociety experiments. We agree that theory of mind is a complex cognitive ability with many different facets (Quesque \& Rossetti, in press). We also appreciate Weinstein and Baldwin's argument that an absence of a statistical link between cumulative performance and theory-of-mind skills as assessed with these two tasks does not imply that this link does not exist, particularly if these tasks are not appropriate for assessing theory of mind. Nevertheless, an important finding is that we obtained such a link in a communication condition in which the participants sat on two chairs back-to-back so that they could only communicate verbally (Osiurak et al. 2020a). As detailed in sect. 3.3, our approach specifically predicts this link in this condition. In broad terms, this result provides strong support for our approach and also confirms that our methodological choice was appropriate. It should also be noted that, as pointed out by Weinstein and Baldwin, we know that the Reading-the-Mind-in-the Eyes task can be subject to ceiling effects. This explains why we proposed a modified version with a time limit of $3 \mathrm{~min}$, allowing us to obtain very sensitive measures even with a population of undergraduate students.

\section{R9. Elephant...action!}

The technical-reasoning hypothesis was initially developed in the field of neuropsychology to account for tool-use disorders observed in apraxic patients. This hypothesis is an alternative to the long-standing hypothesis that tool use is only based on specific tool-use motor programs (see sect. 2.1). This has generated an intense debate in the field, which has led us to discuss in more detail the interaction between technical reasoning and the motor-control system. Some commentaries echo this debate (Houkes \& Vaesen; Lockman, Tamis-LeMonda, \& Adolph [Lockman et al.]; Motes-Rodrigo et al.; Robertson).

The technical-reasoning hypothesis assumes that technical reasoning and the motor-control system are orthogonal processes which have evolved to solve distinct problems. The former generates potential mechanical actions to solve technical problems. The latter selects the most appropriate motor actions to realize the mechanical actions generated through technical reasoning. ${ }^{6}$ Therefore, technical reasoning is concerned with the understanding of mechanical actions, whereas the motor-control system is concerned with the perception of affordances (Osiurak \& Badets 2016; Osiurak et al. 2010; 2017). We define affordances as animalrelative biomechanical properties specifying action possibilities within a body-centered frame of reference (Osiurak et al. 2017). Affordances differ from mechanical actions because mechanical actions are not body-centered but environment-centered. It is one thing to perceive that a knife is grasp-able (i.e., a relationship between the biomechanical property of a given individual and an environmental object) and another to understand that this knife can be useful to cut a tomato (i.e., a relationship between the physical properties of the knife and the tomato). Lockman et al. state that our description of the motor-control system places exaggerated emphasis on the "executive component" of this system and neglects the "explorative component." We strongly agree with this statement in that the motor-control system can also be involved in the exploration of the physical world to extract information useful for both the perception of affordances and the understanding of mechanical actions. We recently elaborated in more detail on this point (Osiurak et al. 2020b).

Robertson holds that our approach needs compelling evidence to demonstrate that mechanical knowledge is not a "dispositional competence" but a kind of "conceptual, propositional knowledge." For him, we "over-intellectualize" the cognitive activity involved in skilled tool practices. Robertson's approach seems to be anchored in an embodied cognition view, as illustrated by his reference to procedural knowledge, a concept close to that of tool-use motor programs. His view is relatively consistent perhaps paradoxically - with the classical distinction between declarative memory and procedural memory, which implies that knowledge is necessarily declarative or "propositional." The fact is that we have never stated that mechanical knowledge is declarative or propositional (sect. 2.1). It is a specific form of knowledge based on an implicit understanding of the physical world which is difficult to make explicit. The corollary is that mechanical knowledge is also not a kind of procedural knowledge based on sensorimotor information as suggested by Robertson. We have already gathered a large body of evidence in favor of this view (for reviews, e.g., Osiurak \& Badets 2016; Osiurak \& Heinke 2018). One of our most compelling arguments is that apraxic patients with tool-use disorders can be impaired not only when asked to use familiar tools, but also when they have to use or make novel tools to solve mechanical problems (see sect. 2.1 and Fig. 2). This finding cannot be interpreted as a deficit of "procedural knowledge" or "tool-use motor programs" because the impairment concerns both the selection of appropriate tools and the realization of the appropriate mechanical action. In other words, the evidence seems to contradict the "under"intellectualized approach to tool use advocated by Robertson.

In the target article, we presented evidence indicating that imitation is not unique to humans and can also be observed in "two-action" paradigms proposed to nonhumans such as 
chimpanzees (e.g., Horner et al. 2006). Motes-Rodrigo et al. claim that these studies do not really demonstrate that chimpanzees can imitate. For instance, they cite the study of Tennie et al. (2010) who presented chimpanzees with a floating peanut task in three conditions. In the full-model condition, chimpanzees could observe a conspecific solving the task by spitting water into the tube. In the "emulation" condition, a human experimenter poured water into the tube. In the baseline condition, there was no demonstration. They found that chimpanzees in the full-model and "emulation" conditions outperformed those in the baseline condition. However, no difference was reported between these two experimental conditions. Although these findings are interesting, they are unfortunately based on a null result. We acknowledge that this null result nevertheless questions the idea that imitation can be found in nonhumans. Nevertheless, as discussed in sect. 2.4 , it appears more viable theoretically to explore imitative skills by placing the focus on the mechanical level instead of the motor level, as proposed in the "two-action" paradigm.

\section{R10. Conclusion}

Similar to any theory, the approach developed in the target article is necessarily incomplete, and, as all scientists are, we are well aware of this. Theories are attempts to organize a collection of findings in a coherent way to offer a satisfying answer to a question, until another theory provides a more satisfying one. Our theoretical approach is no more and no less than such an attempt. In this regard, we are also aware of some points raised by commentators that pose a great challenge to our approach. We agree that our approach to human cultural evolution is too narrow. We focused on cumulative technological culture, whereas human culture is not only technological, but also, for instance, mathematical, artistic or linguistic (Miu et al.; Thornton et al.; Whiten). Of course, we made it clear that our scope was limited to this specific domain. Nevertheless, this limitation could have led us to ignore important aspects that could deeply change our approach. Further work is needed to determine whether this is the case.

We also acknowledge that some findings do not confirm our key assumptions, such as the presence of signs of CTC in New Caledonian crows (sect. R7; Rutz \& Hunt; Taylor \& Jelbert). Other commentators have also stressed that nonhumans might possess cognitive components of technical reasoning (e.g., transfer skills), contrary to what we have assumed (Boesch; Whiten). In this respect, we agree that our theoretical approach needs additional attention to determine whether these findings clearly contradict it. Whiten raised the question of why chimpanzees do not show overimitation in the same way that children or adults do, a finding that could rule out the idea that humans alone possess technical-reasoning skills (see also Thornton et al.). As explained in sect. 4.4, this phenomenon seems to be reported only when children interact with adults, but not with other children. In other words, it is not inconsistent with the idea that children could already possess a certain level of technical reasoning allowing them to detect what is or is not relevant (according to their own level of mechanical knowledge) in the mechanical actions observed. Nevertheless, the development of metacognitive/ theory-of-mind skills could lead them to "blanket-copy" individuals who appear to them as more reliable sources. ${ }^{7}$ Regardless, we agree that empirical effort is needed here to explore this possibility.

To conclude, we would like to address Boesch's skepticism toward the scientific nature of our approach. What is science?
Science does not consist of collecting evidence. This is because of the simple fact that there is no evidence, strictly speaking, but only interpretations of what might constitute evidence. In this context, advances can also come from understanding how scientists think and interpret data to overcome epistemological obstacles. Boesch's commentary is instructive in this way because he seems to impute incorrect motives to our approach. We did not assume that humans are superior by suggesting that they might possess unique non-social cognitive skills. Considering that, say, dolphins or birds possess unique adaptations to hydrodynamical or aerodynamical constraints does not amount to considering that dolphins or birds are superior. We are not ideological, and we did not perpetuate "the simple trick to make humans look superior" (p. 20; Boesch), because, quite simply, we do not view human cognition as superior. Instead, our approach is scientific because it provides an original framework which is intended to overcome a potential epistemological obstacle that consists of thinking that CTC necessarily originates in social cognitive skills. We are convinced that such an alternative approach can be fruitful in providing a reinterpretation of the data collected on the topic. We have already discussed this viewpoint in the different rounds of reviewing we went through with Boesch before publishing our target article. Regardless, this commentary nicely illustrates that CTC is a fascinating phenomenon that needs much more discussion to reconcile the different viewpoints without, nevertheless, imputing incorrect motives to other researchers.

\section{Notes}

1 We acknowledge that we did not mention these important prerequisites in the target article, which could have led some commentators to misinterpret our approach.

2 The concept of strategy implies that the decision is made by the individual and not imposed by the environment. For instance, in the context of CTC, this would consist of opting for social learning instead of asocial learning. Derex and Boyd claim that individuals have to follow such strategies to allocate their time and effort efficiently. To support their claim, they cited the study of Beck et al. (2011), which demonstrated that children can experience difficulties in acquiring a tool-making technique by themselves, but not after social demonstration. The question is whether this study really supports their claim. In this study, the children did not decide to follow the social demonstration instead of addressing the problem themselves. The social demonstration was imposed by the experimenter.

3 Miu et al. pointed out that CTC changes the nature of the technical problems that must be solved, thereby opening up new niches with new technical problems. We fully agree with them and would like to add that this also helps increase the number of techniques discovered and, as a result, reduces the possibility that an individual may, in her or his lifetime, master all the techniques of a given domain and, even less so, multiple domains.

4 As mentioned in sect. 5, we adopted a Lorentzian view in assuming that our "motivation" to solve technical problems comes first and foremost from the very ability to solve technical problems. Therefore, even if the pride hypothesis developed by Tracy is very original, allowing us to build an interesting bridge with the social-learning strategies discussed in sect. 4 , this hypothesis cannot explain the origins of human materiality stricto sensu.

5 Gruber discusses several studies reporting the acquisition of "opaque" toolmaking through social learning in nonhuman primates (e.g., Gruber et al. 2009). According to him, these findings contradict the idea that opacity is the key driver of CTC in humans as suggested by Csibra and Gergely (2009) and, to a lesser extent, by our approach. It is true that we posited that the complexification of tools and their increasing opacity could have favored the emergence of theory-of-mind skills in human ancestors, thereby facilitating social transmission, a perspective similar to that of Csibra and Gergely (2009). However, this implies that the tool repertoire is relatively large and also includes many "opaque" tools. In other words, the observation of some 
instances of opaque tools acquired through social learning does not contradict our approach.

6 In this framework, performing, for instance, a "rotating" motor action to use a screwdriver does not imply that the "rotating" mechanical action involving the screwdriver is not "too far above the level of motor action" (Houkes \& Vaesen, p. 30). This tool-use situation makes it necessary to solve two distinct problems, the first being to determine how to drive the screw with the screwdriver (technical reasoning) and the second to determine which motor action is the most appropriate to do so (motor-control system). Simply, the fact that the realization of the mechanical action imposes a motor action that is very similar can create the illusion that the knowledge of how to use a screwdriver is sensorimotor or procedural.

7 In a way, the interaction described here between technical-reasoning and theory-of-mind skills is consistent with the mechanistic versus mentalistic cognition distinction proposed by Crespi.

Acknowledgments. We thank Tim Pownall for the English proofreading of this manuscript. This work was supported by grants from ANR (Agence Nationale pour la Recherche; Project "Cognition and tool-use economy" ECOTOOL; ANR-14-CE30-0015-01), and was performed within the framework of the LABEX CORTEX (ANR-11-LABX-0042) of Universite de Lyon, within the program "Investissements d'Avenir" (ANR-11- IDEX-0007) operated by the French National Research Agency (ANR).

\section{Conflict of interest. None.}

\section{References}

[The letters " $a$ " and " $r$ " before author's initials stand for target article and response references, respectively]

Abu-Akel, A. M., Wood, S. J., Hansen, P. C. \& Apperly, I. A. (2015) Perspective-taking abilities in the balance between autism tendencies and psychosis proneness Proceedings of the Royal Society B: Biological Sciences 282(1808):20150563. Available at: https://doi.org/10.1098/rspb.2015.0563. [BJC]

Adolph, K. E. \& Robinson, S. R. (2015) Motor development. In: Handbook of child psychology and developmental science, 7th ed., vol. 2, Cognitive Processes, eds. L. Liben \& U. Muller, pp. 113-57. Wiley. [JJL]

Aerts, D. \& Gabora, L. (2005a) A state-context-property model of concepts and their combinations I: The structure of the sets of contexts and properties. Kybernetes 34 (1 \& 2):167-91. [LG]

Aerts, D. \& Gabora, L. (2005b) A state-context-property model of concepts and their combinations II: A Hilbert space representation. Kybernetes 34(1 \& 2):192-221. [LG]

Aerts, D. \& Sozzo, S. (2014) Quantum entanglement in concept combinations. International Journal of Theoretical Physics 53(10):3587-603. [LG]

Allen, C. (2002) A skeptic's progress. Biology and Philosophy 17:695-702. [CB]

Alzheimer's Disease International. (2018) World Alzheimer report 2018: The state of the art of dementia research: New frontiers. Alzheimer's Disease International. [JFB]

Arbib, M. A., Bonaiuto, J. B., Jacobs, S. \& Frey, S. H. (2009) Tool use and the distalization of the end-effector. Psychological Research 73:441-62. [PG]

Ashton-James, C. E. \& Tracy, J. L. (2012) Pride and prejudice: How feelings about the self influence judgments of others. Personality and Social Psychology Bulletin 38:466-76. [JLT]

Atkinson, C., O’Brien, M. J. \& Mesoudi, A. (2012) Adult learners in a novel environment use prestige-biased social learning. Evolutionary Psychology 10:519-37. [aFO, MJO]

Aviezer, H., Ensenberg, N. \& Hassin, R. R. (2017) The inherently contextualized nature of facial emotion perception. Current Opinion in Psychology 17:47-54. [NW]

Bae, C. J., Douka, K. \& Petraglia, M. D. (2017) On the origins of modern humans: Asian perspectives. Science (New York, N.Y.) 358. doi: 10.1126/science.aai9067. [MJO]

Baillargeon, R. \& Hanko-Summers, S. (1990) Is the top object adequately supported by the bottom object? Young infants' understanding of support relations. Cognitive Development 5:29-53. [aFO]

Baillargeon, R., Needham, A. \& DeVos, J. (1992) The development of young infants' intuitions about support. Early Development and Parenting 1:69-78. [aFO]

Bandini, E. \& Tennie, C. (2017) Spontaneous reoccurrence of 'scooping,' a wild tool-use behaviour, in naïve chimpanzees. Peer J 5:e3814. [aFO]

Bania, A., Harris, S., Kinsley, H. \& Boysen, S. (2009) Constructive and deconstructive too modification by chimpanzees (Pan troglodytes). Animal Cognition 12:85-95. [CB]

Bard, K. \& Leavens, D. (2014) The importance of development for comparative primatology. Annual Review of Anthropology 43:183-200. [CB]

Baron-Cohen, S. (1993) Are children with autism acultural? Behavioral and Brain Sciences 16(3):512-13. Available at: https://doi.org/10.1017/S0140525X00031253. [BJC]
Baron-Cohen, S. (2000) Autism: Deficits in folk psychology exist alongside superiority in folk physics. In: Understanding other minds: Perspectives from developmental cognitive neuroscience, eds. S. Baron-Cohen, H. Tager-Flusberg \& D. J. Cohen, pp. 73-82. Oxford University Press. [BJC]

Baron-Cohen, S. (1998) Does autism occur more often in families of physicists, engineers, and mathematicians? Autism 2(3):296-301. Available at: https://doi.org/10.1177/ 1362361398023008. [BJC]

Baron-Cohen, S., Lombardo, M. V., Auyeung, B., Ashwin, E., Chakrabarti, B. \& Knickmeyer, R. (2011) Why are autism spectrum conditions more prevalent in males? PLoS Biology 9:e1001081. Available at: https://doi.org/10.1371/journal.pbio. 1001081. [BJC]

Baron-Cohen, S., Wheelwright, S., Burtenshaw, A. \& Hobson, E. (2007) Mathematical talent is linked to autism. Human Nature 18:125-31. Available at: https://doi.org/10. 1007/s12110-007-9014-0. [BJC]

Baron-Cohen, S., Wheelwright, S., Spong, A., Scahill, V. \& Lawson, J. (2001) Are intuitive physics and intuitive psychology independent? A test with children with Asperger Syndrome. Journal of Developmental and Learning Disorders 5(1):47-78. [BJC]

Baron-Cohen, S., Wheelwright, S., Stott, C., Bolton, P. \& Goodyer, I. (1997) Is there a link between engineering and autism? Autism 1:101-109. Available at: https://doi.org/10. 1177/1362361397011010. [BJC]

Barrett, H. C., Cosmides, L. \& Tooby, J. (2007) The hominid entry into the cognitive niche. In: Evolution of mind, fundamental questions and controversies, eds. S. W. Gangestad \& J. A. Simpson, pp. 241-48. The Guilford Press. [PG]

Barrouillet, P., Portrat, S. \& Camos, V. (2011) On the law relating processing to storage in working memory. Psychological Review 118:175-92. [rFO]

Bartolo, A., Daumüller, M., Della Sala, S. \& Goldenberg, G. (2007) Relationship between object-related gestures and the fractionated object knowledge system. Behavioural Neurology 18:143-47. [aFO]

Bateson, G. (1979) Mind and nature: A necessary unity, vol. 255. Bantam Books. [LG]

Baumard, J., Lesourd, M., Jarry, C., Merck, C., Etcharry-Bouyx, F., Chauviré, V., Belliard, S., Moreaud, O., Croisile, B., Osiurak, F. \& Le Gall, D. (2016) Tool use disorders in neurodegenerative diseases: Roles of semantic memory and technical reasoning. Cortex 82:119-32. [arFO]

Baumard, J., Osiurak, F., Lesourd, M. \& Le Gall, D. (2014) Tool use disorders after left brain damage. Frontiers in Psychology 5:473. [aFO]

Beck, S. R., Apperly, I. A., Chappell, J., Guthrie, C. \& Cutting, N. (2011) Making tools isn't child's play. Cognition 119(2):301-306. Available at: https://doi.org/10.1016/j.cognition.2011.01.003. [arFO, AT, MD]

Beck, S. R., Cutting, N., Apperly, I. A., Demery, Z., Iliffe, L., Rishi, S. \& Chappell, J. (2014) Is tool-making knowledge robust over time and across problems? Frontiers in Psychology 5:1395. [ERRB]

Beck, S. R., Williams, C., Cutting, N., Apperly, I. A. \& Chappell, J. (2016) Individual differences in children's innovative problem-solving are not predicted by divergent thinking or executive functions. Philosophical Transactions of the Royal Society B: Biological Sciences 371(1690):20150190. Available at: https://doi.org/10.1098/rstb.2015.0190. [BR, ERRB]

Benge, J. F., Dinh, K. L., Logue, E., Phenis, R., Dasse, M. N. \& Scullin, M. K. (2020) The smartphone in the memory clinic: A study of patient and care partner's utilisation habits. Neuropsychological Rehabilitation 30(1):101-115. [JFB]

Bentley-Condit, V. K. \& Smith, E. O. (2010) Animal tool use: Current definitions and an updated comprehensive catalog. Behaviour 147:185-221. [aFO]

Binnie, L. \& Williams, J. (2003) Intuitive psychology and physics among children with autism and typically developing children. Autism 7(2):173-93. Available at: https:// doi.org/10.1177/1362361303007002005. [BJC]

Birch, S. A., Akmal, N. \& Frampton, K. L. (2010) Two-year-olds are vigilant of others' non-verbal cues to credibility. Developmental Science 13:363-69. [JLT]

Blair, C. (2006) How similar are fluid cognition and general intelligence? A developmental neuroscience perspective on fluid cognition as an aspect of human cognitive ability. Behavioral and Brain Sciences 29:109-60. [aFO]

Bluff, L. A., Kacelnik, A. \& Rutz, C. (2010a) Vocal culture in New Caledonian crows Corvus moneduloides. Biological Journal of the Linnean Society 101:767-76. [CR]

Bluff, L. A., Troscianko, J., Weir, A. A. S., Kacelnik, A. \& Rutz, C. (2010b) Tool use by wild New Caledonian crows Corvus moneduloides at natural foraging sites. Proceedings of the Royal Society B 277:1377-85. [CR]

Bluff, L. A., Weir, A. A. S., Rutz, C., Wimpenny, J. H. \& Kacelnik, A. (2007) Tool-related cognition in New Caledonian crows. Comparative Cognition \& Behavior Reviews 2:125. [CR]

Boesch, C. (1991) Teaching among wild chimpanzees. Animal Behaviour 41:530-32. [aFO, CB]

Boesch, C. (2002) Cooperative hunting roles among Taï chimpanzees. Human Nature 13:27-46. [aFO]

Boesch, C. (2007) What makes us human (Homo sapiens)? The challenge of cognitive cross-species comparison. Journal of Comparative Psychology 121:227-40. [CB]

Boesch, C. (2012) Wild cultures: A comparison between chimpanzee and human cultures. Cambridge University Press. [CB] 
Boesch, C. \& Boesch, H. (1983) Optimization of nut-cracking with natural hammers by wild chimpanzees. Behaviour 83:265-86. [CB]

Boesch, C. \& Boesch, H. (1984) Mental map in wild chimpanzees: An analysis of hammer transports for nut cracking. Primates 25:160-70. [aFO, CB]

Boesch, C. \& Boesch, H. (1989) Hunting behavior of wild chimpanzees in the Tai National Park. American Journal of Physical Anthropology 78:547-73. [aFO]

Boesch, C. \& Boesch, H. (1990) Tool use and tool making in wild chimpanzees. Folia Primatologica 54:86-99. [CB]

Boesch, C. \& Boesch-Achermann, H. (2000) The chimpanzees of the Tai forest: Behavioural ecology and evolution. Oxford University Press. [aFO]

Boesch, C., Bombjaková, D., Boyette, A. \& Meier, A. (2017) Technical intelligence and culture: Nut cracking in humans and chimpanzees. American Journal of Physical Anthropology 163:339-55. [aFO]

Boesch, C., Bombjakova, D., Meier, A. \& Mundry, R. (2019) Learning curves and teaching when acquiring nut-cracking in humans and chimpanzees. Scientific Reports 9:1515. [aFO, CB]

Boesch, C., Head, J. \& Robbins, M. (2009) Complex toolsets for honey extraction among chimpanzees in Loango National Park, Gabon. Journal of Human Evolution 56:56069. [CB]

Boesch, C. \& Tomasello, M. (1998) Chimpanzee and human cultures. Current Anthropology 39:591-604. [aFO]

Bonnie, K. E., Horner, V., Whiten, A. \& de Waal, F. B. M. (2007) Spread of arbitrary customs among chimpanzees: A controlled experiment. Proceedings of the Royal Society $B$ 274:367-72. [aFO]

Boogert, N. J., Arbilly, M., Muth, F. \& Seed, A. M. (2013) Do crows reason about causes or agents? The devil is in the controls. Proceedings of the National Academy of Sciences of the United States of America 110(4):E273. [AHT, CR]

Bowers, J. S. \& Davis, C. J. (2012) Bayesian just-so stories in psychology and neuroscience. Psychological Bulletin 138(3):389. [IR]

Bowers, K. S., Farvolden, P. \& Mermigis, L. (1995) Intuitive antecedents of insight. The Creative Cognition Approach, 27-51. [LG]

Boyd, R. (2013) The cultural evolution of technologies. In: Cultural evolution: Society, technology, language, and religion, vol. 12, eds. P. J. Richerson \& M. H. Christiansen. MIT Press. [NC]

Boyd, R. \& Richerson, P. J. (1985) Culture and the evolutionary process. University of Chicago Press. [aFO, CK, AT]

Boyd, R. \& Richerson, P. J. (1996) Why culture is common, but cultural evolution is rare. Proceedings of the British Academy 88:77-93. [aFO, CR, AT]

Boyd, R., Richerson, P. J. \& Henrich, J. (2011) The cultural niche: Why social learning is essential for human adaptation. Proceedings of the National Academy of Sciences of the United States of America 108(Suppl. 2):10918-25. Available at: https://doi.org/10. 1073/pnas.1100290108. [arFO, EM, MD, PG, NW]

Boyd, R., Richerson, P. J. \& Henrich, J. (2013) The cultural evolution of technology: Facts and theories. In: Cultural evolution: Society, technology, language, and religion, eds. P. J. Richerson \& M. H. Christiansen, pp. 119-42. MIT Press. [aFO]

Boyd, R. T. (2017) A different kind of animal: How culture transformed our species, vol. 46. Princeton University Press. [EM]

Boyer, P. (2001) Religion explained: The evolutionary origins of religious thought. Basic Books. [aFO]

Brass, M. \& Heyes, C. (2005) Imitation: Is cognitive neuroscience solving the correspondence problem? Trends in Cognitive Sciences 9:489-95. [aFO]

Bril, B., Rein, R., Nonaka, T., Wenban-Smith, F. \& Dietrich, G. (2010) The role of expertise in tool use: Skill differences in functional action adaptations to task constraints. Journal of Experimental Psychology: Human Perception and Performance 36:825-39. [aFO]

Brooks, R. \& Meltzoff, A. N. (2015) Connecting the dots from infancy to childhood: A longitudinal study connecting gaze following, language, and explicit theory of mind. Journal of Experimental Child Psychology 130:67-78. [ASH]

Brosseau-Liard, P., Cassels, T. \& Birch, S. (2014) You seem certain but you were wrong before: Developmental change in preschoolers' relative trust in accurate versus confident speakers. PLoS One 9:e108308. [JLT]

Brosseau-Liard, P. E. \& Poulin-Dubois, D. (2014) Sensitivity to confidence cues increases during the second year of life. Infancy 19:461-75. [JLT]

Brown, C. \& Laland, K. N. (2003) Social learning in fishes: A review. Fish and Fisheries 4:280-88. [MJO]

Bruner, E. (2018) Human paleoneurology and the evolution of the parietal cortex. Brain Behavior and Evolution 91:136-47. [TG]

Bruner, E. \& Lozano Ruiz, M. (2014) Extended mind and visuo-spatial integration: Three hands for the Neandertal lineage. Journal of Anthropological Sciences 92:273-80. [PG] Bruner, J. S. (1983) Child's talk. Norton. [aFO]

Bruza, P. D., Kitto, K., Ramm, B., Sitbon, L., Song, D. \& Blomberg, S. (2012) Quantum-like non-separability of concept combinations, emergent associates and abduction. Logic Journal of the IGPL 20(2):445-57. [LG]

Buchanan, K., Grindstaff, J. \& Pravosudov, V. (2013) Condition dependence, developmental plasticity, and cognition: Implications for ecology and evolution. Trends in Ecology and Evolution 28:290-96. [CB]
Butler, L. P., Ronfard, S. \& Corriveau, K. H., eds. (2020) The questioning child: Insights from psychology and education. Cambridge University Press. [ASH]

Butterworth, G. (2003) Pointing is the royal road to language for babies. In: Pointing: Where language, culture, and cognition meet, ed. S. Kita, pp. 9-33. Lawrence Erlbaum Associates Publishers. [ASH]

Buxbaum, L. J. (2001) Ideomotor Apraxia: A call to action. Neurocase 7:445-48. [aFO] Byrne, R. (1997) The technical intelligence hypothesis: An additional evolutionary stimulus to intelligence? In: Machiavellian intelligence II: Extensions and evaluations, eds. A. Whiten \& W. Byrne, pp. 289-311. Cambridge University Press. [CB]

Byrne, R. \& Whiten, A. (1989) Machiavellian intelligence: Social expertise and the evolution of intellect in monkeys, apes and humans. Oxford Science Publishing. [CB]

Byrne, R. W. \& Whiten, A., eds. (1988) Machiavellian intelligence: Social expertise and the evolution of intellect in monkeys, apes and humans. Clarendon Press. [NC]

Caldwell, C. A., Atkinson, M., Blakey, K. H., Dunstone, J., Kean, D., Mackintosh, G. Renner, E. \& Wilks, C. E. H. (2019) Experimental assessment of capacities for cumulative culture: Review and evaluation of methods. Wiley Interdisciplinary Reviews: Cognitive Science, e1516. [aFO, MJO]

Caldwell, C. A. \& Millen, A. E. (2008) Experimental models for testing hypotheses about cumulative cultural evolution. Evolution and Human Behavior 29:165-71. [aFO]

Caldwell, C. A. \& Millen, A. E. (2009) Social learning mechanisms and cumulative cultural evolution: Is imitation necessary? Psychological Science 20(12):1478-83. [aFO, AT]

Caldwell, C. A. \& Millen, A. E. (2010) Human cumulative culture in laboratory: Effects of (micro) population size. Learning and Behavior 38:310-28. [aFO]

Caldwell, C. A., Renner, E. \& Atkinson, M. (2018) Human teaching and cumulative cultural evolution. Review of Philosophy and Psychology 9(4):751-70. Available at: https://doi.org/10.1007/s13164-017-0346-3. [aFO, AT]

Caldwell, C. A., Schillinger, K., Evans, C. L. \& Hopper, L. M. (2012) End state copying by humans (Homo sapiens): Implications for a comparative perspective on cumulative culture. Journal of Comparative Psychology 126:161-69. [aFO]

Call, J. (2013) Three ingredients for becoming a creative tool user. In: Tool use in animals: Cognition and ecology, eds. C. M. Sanz, J. Call \& C. Boesch, pp. 3-20. Cambridge University Press. [TG]

Call, J. \& Tomasello, M. (1995) Use of social information in the problem solving of orangutans (Pongo pygmaeus) and human children (Homo sapiens). Journal of Comparative Psychology 109(3):308. doi: 10.1037/0735-7036.109.3.308. [HM]

Callanan, M. A. \& Jipson, J. L. (2001). Explanatory conversations and young children's developing scientific literacy. In: Designing for science: Implications from everyday, classroom, and professional settings, eds. K. Crowley, C. D. Schunn \& T. Okada, pp. 21-49, Erlbaum Associates Publishers. [ASH]

Callanan, M. A. \& Oakes, L. M. (1992) Preschoolers questions and parents' explanations: Causal thinking in everyday activity. Cognitive Development 7(2):213-33. [ASH]

Campbell, B. C. \& Wang, S. S. H. (2012) Familial linkage between neuropsychiatric disorders and intellectual interests. PLoS One 7:e30405. Available at: https://doi.org/10. 1371/journal.pone.0030405. [BJC]

Cardoso, R. M. \& Ottoni, E. B. (2016) The effects of tradition on problem solving by two wild populations of bearded capuchin monkeys in a probing task. Biology Letters 12 (11). doi: 10.1098/rsbl.2016.0604. [TG]

Caro, T. M. \& Hauser, M. D. (1992) Is there teaching in nonhuman animals? Quarterly Review of Biology 67:151-74. [aFO]

Carpendale, J. \& Lewis, C. (2004) Constructing an understanding of mind: The development of children's social understanding within social interaction. Behavioral and Brain Sciences 27:79-151. [CB]

Carr, K., Kendal, R. L. \& Flynn, E. G. (2015) Imitate or innovate? Children's innovation is influenced by the efficacy of observed behaviour. Cognition 142:322-32. Available at: https://doi.org/10.1016/j.cognition.2015.05.005. [BR, ERRB]

Carr, K., Kendal, R. L. \& Flynn, E. G. (2016) Eureka!: What is innovation, how does it develop, and who does it? Child Development 87(5):1505-19. Available at: https:// doi.org/10.1111/cdev.12549. [BR, ERRB]

Carruthers, P. (2009) How we know our own minds: The relationship between mindreading and metacognition. Behavioral and Brain Sciences 32:121-38. [aFO]

Casler, K. \& Kelemen, D. (2007) Reasoning about artifacts at 24 months: The developing teleo-functional stance. Cognition 103(1):120-30. [MH]

Chappell, J., Cutting, N., Apperly, I. A. \& Beck, S. R. (2013) The development of tool manufacture in humans: What helps young children make innovative tools? Philosophical Transactions of the Royal Society B: Biological Sciences 368:20120409. [ERRB]

Chappell, J. \& Kacelnik, A. (2002) Tool selectivity in a non-primate, the New Caledonian crow (Corvus moneduloides). Animal Cognition 5:71-78. [CR, JJL]

Chappell, J. \& Kacelnik, A. (2004) Selection of tool diameter by New Caledonian crows Corvus moneduloides. Animal Cognition 7:121-27. [CR]

Charman, T. (2003) Why is joint attention a pivotal skill in autism? Philosophical Transactions of the Royal Society of London. Series B: Biological Sciences 358(1430):315-24. Available at: https://doi.org/10.1098/rstb.2002.1199. [BJC]

Charness, N. \& Boot, W. R. (2009) Aging and information technology use: Potential and barriers. Current Directions in Psychological Science 18(5):253-58. [JFB] 
Chase, W. G. \& Simon, H. A. (1973) Perception in chess. Cognitive Psychology 4:55-81. [aFO]

Chen, Z. \& Klahr, D. (1999) All other things being equal: Acquisition and transfer of control of variables strategy. Child Development 70:1098-1120. [ERRB]

Cheng, J. T., Tracy, J. L. \& Henrich, J. (2010) Pride, personality, and the evolutionary foundations of human social status. Evolution and Human Behavior 31:334-47. [JLT]

Chomsky, N. (2008) On phases. In: Foundational issues in linguistic theory: Essays in honor of Jean-Roger Vergnaud, eds. R. Freidlin, C. P. Otero \& M. L. Subizarreta, pp. 133-66. [LG]

Chouinard, M. M. (2007) Children's questions: A mechanism for cognitive development Monographs of the Society for Research in Child Development 72(1):1-112. [ASH]

Chudek, M. \& Henrich, J. (2011) Culture-gene coevolution, norm-psychology and the emergence of human prosociality. Trends in Cognitive Sciences 15:218-26. [rFO]

Claidière, N., Scott-Phillips, T. C. \& Sperber, D. (2014a) How Darwinian is cultural evolution? Philosophical Transactions of the Royal Society B: Biological Sciences 369:20130368. [aFO, EM]

Claidière, N., Smith, K., Kirby, S. \& Fagot, J. (2014b) Cultural evolution of systematically structured behaviour in a non-human primate. Proceedings of the Royal Society $B$ 281(1797):20141541. doi: 10.1098/rspb.2014.1541. [aFO, NC]

Claidière, N. \& Sperber, D. (2007) The role of attraction in cultural evolution. Journal of Cognition and Culture 7:89-111. [aFO]

Clark, A. (2013) Whatever next? Predictive brains, situated agents, and the future of cognitive science. Behavioral and Brain Sciences 36(3):181-204. [IR]

Clark, A. \& Chalmers, D. (1998) The extended mind (active externalism). Analysis 58(1):7-19. doi: 10.1111/1467-8284.00096. [CK]

Clarkson, C., Jacobs, Z., Marwick, B., Fullagar, R., Wallis, L., Smith, M., Roberts, R. G., Hayes, E., Lowe, K., Carah, X., Florin, S. A., McNeil, J., Cox, D., Arnold, L. J., Hua Q., Huntley, J., Brand, H. E. A., Manne, T., Fairbairn, A., Shulmeister, J., Lyly, L. Salinas, M., Page, M., Connell, K., Park, G., Norman, K., Murphy, T. \& Pardoe, C (2017) Human occupation of northern Australia by 65,000 years ago. Nature 547:306-10. [MJO]

Clay, Z. \& Tennie, C. (2017) Is over-imitation a uniquely human phenomenon? Insights from human children as compared to bonobos. Child Development 89:1535-44. [AM-R]

Clutton-Brock, T. \& Harvey, P. (1980) Primates, brains and ecology. Journal of Zoology 190:309-23. [CB]

Collard, M., Kemery, M. \& Banks, S. (2005) Causes of toolkit variation among huntergatherers: A test of four competing hypotheses. Canadian Journal of Archaeology 29:1-19. [aFO]

Collard, M., Vaesen, K., Cosgrove, R. \& Roebroeks, W. (2016) The empirical case against the 'demographic turn' in Paleolithic archaeology. Philosophical Transactions of the Royal Society B 371:20150242. [aFO]

Colombetti, G. (2017) Enactive affectivity, extended. Topoi 36(3):445-55. [IR]

Colombi, C., Liebal, K., Tomasello, M., Young, G., Warneken, F. \& Rogers, S. J. (2009) Examining correlates of cooperation in autism: Imitation, joint attention, and understanding intentions. Autism 13(2):143-63. Available at: https://doi.org/10.1177/1362361308098514. [BJC]

Corballis, M. C. (2011) The recursive mind: The origins of human language, thought and civilization. Princeton University Press. [LG]

Corbey, R., Jagich, A., Vaesen, K. \& Collard, M. (2016) The Acheulean handaxe: More like a bird's song than a Beatles' tune? Evolutionary Anthropology 25:6-19. [CR]

Corriveau, K. H., Min, G., Chin, J. \& Doan, S. (2016) Do as I do, not as I say: Children's learning of self-regulatory strategies. Journal of Experimental Child Psychology 143:179-87. [ASH]

Cosmides, L. \& Tooby, J. (2001) Unravelling the enigma of human intelligence Evolutionary psychology and the multimodular mind. In: The evolution of intelligence, eds. R. J. Sternberg \& J. C. Kaufman, pp. 53-115. Erlbaum. [PG]

Cowan, N. (2017) The many faces of working memory and short-term storage. Psychonomic Bulletin \& Review 24:1158-70. [AM-R]

Creanza, N., Kolodny, O. \& Feldman, M. W. (2017) Cultural evolutionary theory: How culture evolves and why it matters. Proceedings of the National Academy of Sciences of the United States of America 114(30):7782-89. doi: 10.1073/pnas.1620732114. [CK]

Crespi, B., Leach, E., Dinsdale, N., Mokkonen, M. \& Hurd, P. (2016) Imagination in human social cognition, autism, and psychotic-affective conditions. Cognition 150:181-99. Available at: https://doi.org/10.1016/j.cognition.2016.02.001. [BJC]

Crespi, B. J. (2004) Vicious circles: Positive feedback in major evolutionary and ecological transitions. Trends in Ecology \& Evolution 19(12):627-33. Available at: https://doi.org/10.1016/j.tree.2004.10.001. [BJC]

Crespi, B. J. (2016) Autism as a disorder of high intelligence. Frontiers in Neuroscience 10:300. Available at: https://doi.org/10.3389/fnins.2016.00300. [BJC]

Crespi, B. J. \& Go, M. C. (2015) Diametrical diseases reflect evolutionary-genetic tradeoffs: Evidence from psychiatry, neurology, rheumatology, oncology and immunology. Evolution, Medicine, and Public Health 2015(1):216-53. Available at: https://doi.org/ 10.1093/emph/eov021. [BJC]

Crowley, K., Callanan, M., Jipson, J. L., Galco, J., Topping, K. \& Shrager, J. (2001a) Shared scientific thinking in everyday parent-child activity. Science Education 85(6):712-32. Available at: https://doi.org/10.1002/sce.1035. [ASH]
Crowley, K., Callanan, M. A., Tenenbaum, H. R. \& Allen, E. (2001b) Parents explain more often to boys than to girls during shared scientific thinking. Psychological Science 12(3):258-61. Available at: https://doi.org/10.1111/1467-9280.00347. [ASH]

Crowley, K. \& Siegler, R. S. (1999) Explanation and generalization in young children's strategy learning. Child Development 70(2):304-16. Available at: https://doi.org/10. 1111/1467-8624.00023. [ASH]

Csibra, G. (2007) Teachers in the wild. Trends in Cognitive Sciences 11:95-96. [aFO]

Csibra, G. \& Gergely, G. (2006) Social learning and social cognition: The case for pedagogy. In: Attention and performance XXI: Processes of change in brain and cognitive development, eds. Y. Munakata \& M. H. Johnson, pp. 249-74. Oxford University Press. [aFO]

Csibra, G. \& Gergely, G. (2007) 'Obsessed with goals': Functions and mechanisms of teleological interpretation of actions in humans. Acta Psychologica 124(1):60-78. [MH]

Csibra, G. \& Gergely, G. (2009) Natural pedagogy. Trends in Cognitive Sciences 13:14853. [arFO, TG]

Csibra, G. \& Gergely, G. (2011) Natural pedagogy as evolutionary adaptation. Philosophical Transactions of the Royal Society B: Biological Sciences 366:1149-57. [aFO, TG]

Cutting, N., Apperly, I. A. \& Beck, S. R. (2011) Why do children lack the flexibility to innovate tools? Journal of Experimental Child Psychology 109:497-511. [rFO]

Cutting, N., Apperly, I. A., Chappell, J. \& Beck, S. R. (2014) The puzzling difficulty of tool innovation: Why can't children piece their knowledge together? Journal of Experimental Child Psychology 125:110-17. [aFO, ERRB]

Cutting, N., Apperly, I. A., Chappell, J. \& Beck, S. R. (2019) Is tool modification more difficult than innovation? Cognitive Development 52:100811. Available at: https://doi. org/10.1016/j.cogdev.2019.100811. [BR, ERRB]

Czaja, S. J., Charness, N., Fisk, A. D., Hertzog, C., Nair, S. N., Rogers, W. A. \& Sharit, J. (2006) Factors predicting the use of technology: Findings from the Center for Research and Education on Aging and Technology Enhancement (CREATE). Psychology and Aging 21(2):333-52. [JFB]

Damian, R. I. \& Robins, R. W. (2013) Aristotle's virtue or Dante's deadliest sin? The influence of authentic and hubristic pride on creative achievement. Learning and Individual Differences 26:156-60. [JLT]

Danel, S., Osiurak, F. \& von Bayern, A. M. P. (2017) From the age of 5 humans decide economically, whereas crows exhibit individual preferences. Scientific Reports 7:17043. [aFO]

Davidson, C. A., Lesser, R., Parente, L. T. \& Fiszdon, J. M. (2018) Psychometrics of social cognitive measures for psychosis treatment research. Schizophrenia Research 193:5157. [NW]

Davis, S. J., Schapiro, S. J., Lambeth, S. P., Wood, L. A. \& Whiten, A. (2018) Behavioral conservatism is linked to complexity of behavior in chimpanzees: Implications for cognition and cumulative culture. Journal of Comparative Psychology 133:20-35. doi: 10.1037/com0000123. [AW]

Davis, S. J., Vale, G. L., Schapiro, S. J., Lambeth, S. P. \& Whiten, A. (2016) Foundations of cumulative culture in apes: Improved foraging efficiency through social learning and the inhibition and combination of behaviours in chimpanzees (Pan troglodytes). Scientific Reports 6:35953. [AW]

Dawkins, R. (1976/2016) The selfish gene. 40th anniversary edition. Oxford University Press. [CK]

Dawkins, R. (1982) Replicators and vehicles. In: Current problems in sociobiology, ed. K. S. C. Sociobiology \& Group, pp. 45-64. Cambridge University Press. [CK]

Dawkins, R. (1983) Universal Darwinism. In: Evolution from molecules to men, ed. D. S. Bendall, pp. 403-25. Cambridge University Press. [CK]

Dean, L. G., Kendal, R. L., Schapiro, S. J., Thierry, B. \& Laland, K. N. (2012) Identification of the social and cognitive processes underlying human cumulative culture. Science (New York, N.Y.) 335(6072):1114-18. doi: 10.1126/science.1213969. [aFO, NC]

Dean, L. G., Vale, G. L., Laland, K. N., Flynn, E. \& Kendal, R. L. (2014) Human cumulative culture: A comparative perspective. Biological Reviews 89:284-301. [aFO, CR]

Deblauwe, I., Guislain, P., Dupain, J. \& van Elsacker, L. (2006) Use of a tool-set by Pan troglodytes troglodytes to obtain termites (Macroterms) in the periphery of the Dja Biosphere Reserve, Southeast Cameroon. American Journal of Primatology 68:119196. [aFO]

Dennett, D. C. (1990) The interpretation of texts, people and other artifacts. Philosophy and Phenomenological Research 50:177-94. [MH]

De Oliveira, E., Reynaud, E. \& Osiurak, F. (2019) Roles of technical reasoning, theory of mind, creativity, and fluid cognition in cumulative technological culture. Human Nature 30:326-40. [arFO, NW]

Derex, M., Beugin, M. P., Godelle, B. \& Raymond, M. (2013a) Experimental evidence for the influence of group size on cultural complexity. Nature 503:389-91. [aFO]

Derex, M., Bonnefon, J. F., Boyd, R. \& Mesoudi, A. (2019) Causal understanding is not necessary for the improvement of culturally evolving technology. Nature Human Behaviour 3:446-52. doi: 10.1038/s41562-019-0567-9. [arFO, MD, NC, PG]

Derex, M. \& Boyd, R. (2015) The foundations of the human cultural niche. Nature Communications 6:8398. [aFO]

Derex, M., Godelle, B. \& Raymond, M. (2013b) Social learners require process information to outperform individual learners. Evolution 67:688-97. [aFO] 
d'Errico, F. \& Banks, W. E. (2015) The archaeology of teaching: A conceptual framework. Cambridge Archaeological Journal 25(4):859-66. [PG]

Diamond, A. (2013) Executive functions. Annual Review of Psychology 64:135-68. [AM-R]

Donald, M. (1991) Origins of the modern mind: Three stages in the evolution of culture and cognition. Harvard University Press. [LG]

Dorfman, A., Eyal, T. \& Bereby-Meyer, Y. (2014) Proud to cooperate: The consideration of pride promotes cooperation in a social dilemma. Journal of Experimental Social Psychology 55:105-109. [JLT]

Dunbar, R. I. M. (1992) Neocortex size as a constraint on group size in primates. Journal of Human Evolution 22(6):469-93. [CB, NC]

Dunbar, R. I. M. (2012) The social brain meets neuroimaging. Trends in Cognitive Sciences 16(2):101-102. Available at: https://doi.org/10.1016/j.tics.2011.11.013. [NC]

Dunstone, J. \& Caldwell, C. A. (2018) Cumulative culture and explicit metacognition: A review of theories, evidence and key predictions. Palgrave Communications 4:145. [aFO]

Duval, C., Bejanin, A., Piolino, P., Laisney, M., de la Sayette, V., Belliard, S., Eustache, F. \& Desgranges, B. (2012) Theory of mind impairments in patients with semantic dementia. Brain 135:228-41. [rFO]

Dymond, S., Haselgrove, M. \& McGregor, A. (2013) Clever crows or unbalanced birds? Proceedings of the National Academy of Sciences of the United States of America 110(5):E336. [AHT, CR]

Earl, J. W. \& McCleary, B. V. (1994) Mystery of the poisoned expedition. Nature 368(6473):683-84. [EM]

Ebel, S. J., Hanus, D. \& Call, J. (2019) How prior experience and task presentation modulate innovation in 6-year-old children. Journal of Experimental Child Psychology 180:87-103. [ERRB]

Ehn, M. \& Laland, K. N. (2012) Adaptive strategies for cumulative cultural learning. Journal of Theoretical Biology 301:103-11. [aFO]

Enquist, M., Eriksson, K. \& Ghirlanda, S. (2007) Critical social learning: A solution to Rogers's paradox of nonadaptive culture. American Anthropologist 109:727-34. [aFO]

Ericsson, K. \& Delaney, P. (1999) Long-term working memory as an alternative to capacity of working memory in everyday skilled performance. In: Models of working memory: Mechanisms of active maintenance and executive control, eds. A. Miyake \& P. Shah, pp. 257-97. Cambridge University Press. [aFO]

Ericsson, K. \& Kintsch, W. (1995) Long-term working memory. Psychological Review 102:211-45. [aFO]

Estienne, V., Cohen, H., Wittig, R. \& Boesch, C. (2019a) Maternal influence on the development of nut-cracking skills in the chimpanzees of the Taï forest, Côte d'Ivoire (Pan troglodytes verus). American Journal of Primatology, e23022. doi: 10.1002/ajp.2302. [CB]

Estienne, V., Robira, B., Mundry, R., Deschner, T. \& Boesch, C. (2019b) Acquisition of a complex extractive technique by the immature chimpanzees of Loango National Park, Gabon. Animal Behaviour 147:61-76. [CB]

Falótico, T. \& Ottoni, E. B. (2014) Sexual bias in probe tool manufacture and use by wild bearded capuchin monkeys. Behavioural Processes 108:117-22. [aFO]

Fares, R., Belmeguenai, A., Sanchez, P., Kouchi, H., Bodennec, J., Morales, A., Georges, B., Bonnet, C., Bouvard, S., Sloviter, R. \& Bezin, L. (2013) Standardized environmental enrichment supports enhanced brain plasticity in healthy rats and prevents cognitive impairment in epileptic rats. PLoS ONE 8:e53888. [CB]

Fauconnier, G. \& Turner, M. (2008) The way we think: Conceptual blending and the mind's hidden complexities. Basic Books. [LG]

Fay, N., De Kleine, N., Walker, B. \& Caldwell, C. A. (2019) Increasing population size can inhibit cumulative cultural evolution. Proceedings of the National Academy of Sciences USA 116:6726-31. [aFO]

Feher, O., Wang, H., Saar, S., Mitra, P. P. \& Tchernichovski, O. (2009) De novo establishment of wild-type song culture in the zebra finch. Nature 459:564-68. doi: 10.1038/ nature07994. [NC]

Flavell, J. H. (1979) Metacognition and cognition monitoring: A new area of cognitive developmental inquiry. American Psychologist 34:906-11. [aFO]

Flavell, J. H. (2000) Development of children's knowledge about the mental world. International Journal of Behavioral Development 24:15-23. [aFO]

Flynn, E. (2008) Investigating children as cultural magnets: Do young children transmit redundant information along diffusion chains? Philosophical Transactions of the Royal Society B 363:3541-51. [aFO]

Flynn, E. \& Whiten, A. (2013) Dissecting children's observational learning of complex actions through selective video displays. Journal of Experimental Child Psychology 116:247-63. [aFO]

Fontenelle, S. A., Kahrs, B. A., Neal, S. A., Newton, A. T. \& Lockman, J. J. (2007) Infant manual exploration of composite substrates. Journal of Experimental Child Psychology 98:153-67. [JJL]

Franks, N. R. \& Richardson, T. (2006) Teaching in tandem-running ants. Nature 439:153. [aFO]

Frazier, B. N., Gelman, S. A. \& Wellman, H. M. (2009) Preschoolers' search for explanatory information within adult-child conversation. Child Development 80(6):1592611. [ASH]
Frey, S. H. (2007) What pus the how in where? Tool use and the divided visual stream hypothesis. Cortex 43:368-75. [aFO]

Fridland, E. (2017) Skill and motor control: Intelligence all the way down. Philosophical Studies 174(6):1539-60. [IR]

Friston, K. (2018) Does predictive coding have a future? Nature Neuroscience 21(8):101921. [IR]

Furlong, E., Boose, K. \& Boysen, S. (2008) Raking it in: The impact of enculturation on chimpanzee tool use. Animal Cognition 11:83-97. [CB]

Gabora, L. (1995) Meme and variations: A computer model of cultural evolution. In: 1993 Lectures in complex systems, eds. L. Nadel \& D. Stein, pp. 471-86. Addison-Wesley. [LG]

Gabora, L. (2008) EVOC: A computer model of the evolution of culture. In: 30th Annual meeting of the cognitive science society, eds. V. Sloutsky, B. Love \& K. McRae, pp. 1466-71. Sheridan Publishing. [LG]

Gabora, L. (2018) The creative process of cultural evolution. In: Handbook of culture and creativity: Basic processes and applied innovations, ed. A. Leung, pp. 33-60. Oxford University Press. [LG]

Gabora, L. (2019) Creativity: Linchpin in the quest for a viable theory of cultural evolution. Current Opinion in Behavioral Sciences 27:77-83. [LG]

Gabora, L. (2020) A complex systems framework for cultural evolution. In: 10th Volume of the annual series on advances in culture and psychology, eds. M. Gelfand, C. Chiu \& Y. Hong. Oxford University Press. [LG]

Gabora, L., Leijnen, S., Veloz, T. \& Lipo, C. (2011) A non-phylogenetic conceptual network architecture for organizing classes of material artifacts into cultural lineages. In: Proceedings of the 33rd annual meeting of the cognitive science society, eds. L. Carlson, C. Hőlscher \& T. F. Shipley, pp. 2923-28. Cognitive Science Society. [LG]

Gabora, L. \& Smith, C. (2018) Two cognitive transitions underlying the capacity for cultural evolution. Journal of Anthropological Sciences 96:27-52. doi: 10.4436/jass. 96008. [LG]

Gabora, L. \& Smith, C. (2019) Exploring the psychological basis for transitions in the archaeological record. In: Handbook of cognitive archaeology: A psychological framework, eds. T. Henley, E. Kardas \& M. Rossano, pp. 218-39. Routledge/Taylor \& Francis. Available at: https://doi.org/10.4324/9780429488818. [LG]

Gabora, L. \& Steel, M. (2017) Autocatalytic networks in cognition and the origin of culture. Journal of Theoretical Biology 431:87-95. [LG]

Gabora, L. \& Steel, M. (under review) An autocatalytic network approach to cultural lineages: Modeling the cognitive transition from Oldowan to Acheulian tool technology. [LG]

Gabora, L. \& Tseng, S. (2017) The social benefits of balancing creativity and imitation: Evidence from an agent-based model. Psychology of Aesthetics, Creativity, and the Arts 11(4):403. [LG]

Galef, B. G., Jr. (1988) Imitation in animals: History, definitions, and interpretation of data from the psychological laboratory. In: Social learning: Psychological and biological perspectives, eds. T. Zentall \& B. Galef, pp. 3-28. Erlbaum. [aFO]

Galef, B. G., Jr. (1992) The question of animal culture. Human Nature 3:157-78. [aFO]

Galef, B. G., Jr. (1995) Why behaviour patterns that animals learn socially are locally adaptive. Animal Behaviour 49:1325-34. [aFO]

Galef, B. G., Jr. (1998) Comments on Boesch and Tomasello 'Chimpanzee and human cultures.' Current Anthropology 39:605-606. [aFO]

Gao, C., Chapagain, N. Y. \& Scullin, M. K. (2019) Sleep duration and sleep quality in caregivers of patients with dementia: A systematic review and meta-analysis. JAMA Network Open 2(8):e199891. [JFB]

Gärdenfors, P. \& Högberg, A. (2017) The archaeology of teaching and the evolution of Homo docens. Current Anthropology 58(2):188-208. [PG]

Gärdenfors, P. \& Lombard, M. (2018) Causal cognition, force dynamics and early hunting technologies. Frontiers in Psychology, 12 February, 2018. doi.org/10.3389/ fpsyg.2018.00087. [PG]

Gatewood, J. (1985) Actions speak louder than words. In: Directions in cognitive anthropology, ed. J. Dougherty, pp. 199-200. University of Illinois Press. [aFO]

Gaver, W. W. (1991) Technology affordances. In: Proceedings of the CHI'91 SIGCHI conference on human factors in computing systems, eds. S. P. Robertson, G. Olson \& J. Olson, pp. 79-84. ACM Press. [JJL]

Gentner, D., Levine, S. C., Ping, R., Isaia, A., Dhillon, S., Bradley, C. \& Honke, G. (2016) Rapid learning in a children's museum via analogical comparison. Cognitive Science 40:224-40. [ERRB]

Gergely, G., Bekkering, H. \& Kiràly, I. (2002) Developmental psychology: Rational imitation in preverbal infants. Nature 415:755. [aFO]

Gergely, G. \& Csibra, G. (2003) Teleological reasoning in infancy: The nave theory of rational action. Trends in Cognitive Sciences 7(7):287-92. [MH]

Gergely, G. \& Csibra, G. (2006) Sylvia's recipe: The role of imitation and pedagogy in the transmission of cultural knowledge. In: Roots of human sociality: Culture, cognition, and human interaction, eds. N. J. Enfield \& S. C. Levenson, pp. 229-55. Berg Publishers. [aFO, MH]

German, T. P. \& Barrett, H. C. (2005) Functional fixedness in a technologically sparse culture. Psychological Science 16(1):1-5. [MH] 
Ghirlanda, S. \& Lind, J. (2017) 'Aesop's fable' experiments demonstrate trial-and-error learning in birds, but no causal understanding. Animal Behaviour 123:239-47. [CR]

Gibson, E. J. (1982) The concept of affordances in development: The renascence of functionalism. In: The concept of development: The Minnesota symposia on child psychol $o g y$, vol. 15, ed. W. A. Collins, pp. 55-81. Lawrence Erlbaum Associates. [JJL]

Gibson, J. J. (1979) The ecological approach to visual perception. Houghton Mifflin. [JJL]

Gibson, K. R. (1993) Generative interplay between technical capacities, social relations, imitation and cognition. In: Tools, language and cognition in human evolution, eds K. R. Gibson \& T. Ingold, pp. 131-37. Cambridge University Press. [aFO]

Giraldeau, L. A., Valone, T. J. \& Templeton, J. J. (2002) Potential disadvantages of using socially acquired information. Philosophical Transactions of the Royal Society B 357:1559-66. [aFO]

Gobet, F. (2016) Understanding expertise: A multi-disciplinary approach. Palgrave. [aFO]

Goldenberg, G. (2013) Apraxia: The cognitive side of motor control. Oxford University Press. [aFO]

Goldenberg, G. \& Hagmann, S. (1998) Tool use and mechanical problem solving in apraxia. Neuropsychologia 36:581-89. [aFO]

Goldenberg, G., Hartmann-Schmid, K., Sürer, F., Daumüller, M. \& Hermsdörfer, J. (2007) The impact of dysexecutive syndrome on use of tools and technical devices. Cortex 43:424-35. [aFO]

Goldenberg, G. \& Spatt, J. (2009) The neural basis of tool use. Brain 132:1645-55. [aFO]

Goodall, J. (1968) Behaviour of free-living chimpanzees of the Gombe Stream area Animal Behaviour Monograph 1:163-311. [CB]

Goodall, J. (1970) Tool-using in primates and other vertebrates. In: Advances in the study of behavior, eds. D. S. Lehrmann, R. A. Hinde \& E. Shaw, vol. 3, pp. 195-249. Academic Press. [CB]

Gopnik, A. (2012) Scientific thinking in young children: Theoretical advances, empirical research, and policy implications. Science (New York, N.Y.) 337:1623-27. [ERRB]

Gopnik, A., Griffiths, T. L. \& Lucas, C. G. (2015) When younger learners can be better (or at least more open-minded) than older ones. Current Directions in Psychological Science 24:87-92. [ERRB]

Gopnik, A., O'Grady, S., Lucas, C. G., Griffiths, T. L., Wente, A., Bridgers, S., Aboody, R., Fung, H. \& Dahl, R. E. (2017) Changes in cognitive flexibility and hypothesis search across human life history from childhood to adolescence to adulthood. Proceedings of the National Academy of Sciences of the United States of America 114:7892-99. [ERRB]

Gopnik, A. \& Wellman, H. M. (1994) The theory. In: Mapping the mind: Domain specificity in cognition and culture, eds. L. A. Hirschfeld \& S. A. Gelman, pp. 257-93. Cambridge University Press. [NW]

Greif, H. (2017) What is the extension of the extended mind? Synthese 194(11):4311-36 doi: 10.1007/s11229-015-0799-9. [CK]

Gruber, R., Schiestl, M., Boeckle, M., Frohnwieser, A., Miller, R., Gray, R. D., Clayton, N. S. \& Taylor, A. H. (2019) New Caledonian crows use mental representations to solve metatool problems. Current Biology 29(4):686-92. [AHT, CR]

Gruber, T. (2016) Great apes do not learn novel tool use easily: Conservatism, functional fixedness, or cultural influence? International Journal of Primatology 37(2):296-316. doi: 10.1007/s10764-016-9902-4. [aFO, TG]

Gruber, T., Frick, A., Hirata, S., Adachi, I. \& Biro, D. (2019) Spontaneous categorization of tools based on observation in children and chimpanzees. Scientific Reports 9(1):1-12. [MH]

Gruber, T., Muller, M. N., Reynolds, V., Wrangham, R. W. \& Zuberbühler, K. (2011) Community-specific evaluation of tool affordances in wild chimpanzees. Scientific Reports 1:128. doi: 10.1038/srep00128. [aFO, TG]

Gruber, T., Muller, M. N., Strimling, P., Wrangham, R. W. \& Zuberbühler, K. (2009) Wild chimpanzees rely on cultural knowledge to solve an experimental honey acquisition task. Current Biology 19:1806-10. [rFO, TG]

Gruber, T., Zuberbühler, K., Clément, F. \& van Schaik, C. (2015) Apes have culture but may not know that they do. Frontiers in Psychology 6:91. [aFO]

Guilford, J. P. (1967) The nature of human intelligence. McGraw-Hill. [aFO]

Hackman, D. \& Farah, M. (2009) Socioeconomic status and the developing brain. Trends in Cognitive Science 13:65-73. [CB]

Haden, C. A. (2010) Talking about science in museums. Child Development 4(1):62-67. [ASH]

Haidle, M. N. (2010) Working-memory capacity and the evolution of modern cognitive potential. Current Anthropology 51:S149-66. [aFO]

Haidle, M. N. (2014) Building a bridge: An archaeologist's perspective on the evolution of causal cognition. Frontiers in Psychology 5:1472. [aFO]

Halford, G. S., Wilson, W. H. \& Phillips, S. (1998) Processing capacity defined by relational complexity: Implications for comparative, developmental, and cognitive psychology. Behavioral and Brain Sciences 21:803-65. [AM-R]

Hampton, J. (1987) Inheritance of attributes in natural concept conjunctions. Memory \& Cognition 15:55-71. [LG]

Hanner, E., Braham, E. J., Elliott, L. \& Libertus, M. E. (2019) Promoting math talk in adult-child interactions through grocery store signs. Mind, Brain, and Education 13(2):110-18. Available at: https://doi.org/10.1111/mbe.12195. [ASH]
Hanus, D., Mendes, N., Tennie, C. \& Call, J. (2011) Comparing the performances of apes (Gorilla gorilla, Pan troglodytes, Pongo pygmaeus) and human children (Homo sapiens) in the floating peanut task. PLoS One 6(6):e19555. doi: 10.1371/journal.pone. 0019555. [HM]

Happé, F. \& Frith, U., eds. (2010) Autism and talent, 364(1522). Oxford University Press. $[\mathrm{BJC}$

Hare, B. (2017) Survival of the friendliest: Homo sapiens evolved via selection for prosociality. Annual Review of Psychology 68:155-86. doi: 10.1146/annurev-psych-010416044201. [CK]

Hare, B., Plyusnina, I., Ignacio, N., Schepina, O., Stepika, A., Wrangham, R. \& Trut, L. (2005) Social cognitive evolution in captive foxes is a correlated by-product of experimental domestication. Current Biology 15(3):226-30. doi: 10.1016/j.cub.2005. 01.040. [CK]

Harris, P. L. (1991) The work of the imagination. In: Natural theories of mind: Evolution, simulation, and development of everyday mindreading, ed. A. Whiten, pp. 283-304. Basil Blackwell. [aFO]

Harris, P. L. (2012) Trusting what you're told: How children learn from others. The Belknap Press/Harvard University Press. [ASH]

Harris, P. L. \& Corriveau, K. H. (2011) Young children's selective trust in informants. Philosophical Transactions of the Royal Society B 366:1179-87. [aFO]

Harris, P. L. \& Corriveau, K. H. (2014) Learning from testimony about religion and science. In: Trust and skepticism: Children's selective learning from testimony, eds. S. Einav \& E. Robinson, pp. 28-41. Psychology Press. [ASH]

Harris, P. L. \& Koenig, M. (2006) Trust in testimony: How children learn about science and religion. Child Development 77(3):505-24. Available at: https://doi.org/10.1111/j. 1467-8624.2006.00886.x. [ASH]

Harris, P. L., Koenig, M. A., Corriveau, K. H. \& Jaswal, V. K. (2018) Cognitive foundations of learning from testimony. Annual Review of Psychology 69:251-73. Available at doi: 10.1146/annurev-psych-122216-011710. [ASH]

Hartmann, K., Goldenberg, G., Daumüller, M. \& Hermsdörfer, J. (2005) It takes the whole brain to make a cup of coffee: The neuropsychology of naturalistic actions involving technical devices. Neuropsychologia 43:625-27. [aFO]

Hauser, M. (2001) Elementary my dear chimpanzee. Science (New York, N.Y.) 291:44041. [CB]

Hauser, M. D., Chomsky, N. \& Fitch, W. T. (2002) The faculty of language: What is it, who has it and how did it evolve? Science (New York, N.Y.) 298:1569-79. [LG]

Hayashi, M., Kato, M., Igarashi, K. \& Kashima, H. (2008) Superior fluid intelligence in children with Asperger's disorder. Brain and Cognition 66(3):306-310. Available at: https://doi.org/10.1016/j.bandc.2007.09.008. [BJC]

Hecht, E. E., Gutman, D. A., Khreisheh, N., Taylor, S. V., Kilner, J., Faisal, A. A., Bradley, B. A., Chaminade, T. \& Stout, D. (2015) Acquisition of Paleolithic toolmaking abilities involves structural remodeling of inferior frontoparietal regions. Brain Structure and Function 220:2315-31. [aFO]

Hegarty, M. (2004) Mechanical reasoning by mental simulation. Trends in Cognitive Sciences 8:280-85. [aFO]

Heilman, K. M., Maher, L. M., Greenwald, M. L. \& Rothi, L. J. G. (1997) Conceptual apraxia from lateralized lesions. Neurology 49:457-64. [aFO]

Heilman, K. M., Rothi, L. J. \& Valenstein, E. (1982) Two forms of ideomotor apraxia. Neurology 32:342-46. [aFO]

Hennefield, L., Hwang, H. G., Weston, S. J. \& Povinelli, D. J. (2018) Meta-analytic techniques reveal that corvid causal reasoning in the Aesop's Fable paradigm is driven by trial-and-error learning. Animal Cognition 21:735-48. [CR]

Henrich, J. (2001) Cultural transmission and the diffusion of innovations: Adoption dynamics indicate that biased cultural transmission is the predominate force in behavioral change. American Anthropologist 103:992-1013. [aFO]

Henrich, J. (2009) The evolution of innovation-enhancing institutions. In: Innovation in cultural systems: Contributions from evolutionary anthropology, eds. S. J. Shennan \& M. J. O’Brian, pp. 99-120. MIT Press. [CK]

Henrich, J. (2016) The secret of our success: How culture is driving human evolution, domesticating our species, and making us smarter. Princeton University Press. [CK, EM, JT]

Henrich, J. (2017) The secret of our success: How culture is driving human evolution, domesticating our species, and making us smarter. Princeton: Princeton University Press. [JLT]

Henrich, J., Boyd, R. \& Richerson, P. J. (2008) Five misunderstandings about cultural evolution. Human Nature (Hawthorne, N.Y.) 19(2):119-37. doi: 10.1007/s12110-0089037-1. [CK]

Hernik, M. \& Csibra, G. (2009) Functional understanding facilitates learning about tools in human children. Current Opinion in Neurobiology 19(1):34-38. [aFO, MH]

Hernik, M. \& Csibra, G. (2015) Infants learn enduring functions of novel tools from action demonstrations. Journal of Experimental Child Psychology 130:176-92. [MH]

Henrich, J. \& Broesch, J. (2011) On the nature of cultural transmission networks: Evidence from Fijian villages for adaptive learning biases. Philosophical Transactions of the Royal Society B 336:1139-48. [aFO]

Henrich, J. \& Gil-White, F. J. (2001) The evolution of prestige: Freely conferred deference as a mechanism for enhancing the benefits of cultural transmission. Evolution and Human Behavior 22:165-96. [aFO, JLT] 
Henrich, J. \& Tennie, C. (2017) Cultural evolution in chimpanzees and humans. In: Chimpanzees and human evolution, eds. M. Muller, R. Wrangham \& D. Pilbeam. Harvard University Press. [AM-R]

Herrmann, E., Call, J., Hernandez-Lloreda, M., Hare, B. \& Tomasello, M. (2007) Humans have evolved specialized skills of social cognition: The cultural intelligence hypothesis. Science (New York, N.Y.) 317:1360-66. [aFO]

Hewlett, B. S., Fouts, H. N., Boyette, A. H. \& Hewlett, B. L. (2011) Social learning among Congo Basin hunter-gatherers. Philosophical Transactions of the Royal Society B 366:1168-78. [aFO]

Heyes, C. (2012) What's social about social learning? Journal of Comparative Psychology 126(2):193-202. doi: 10.1037/a0025180. [CK, EM]

Heyes, C. (2016) Who knows? Metacognitive social learning strategies. Trends in Cognitive Sciences 20:204-13. [aFO]

Heyes, C. (2018) Enquire within: Cultural evolution and cognitive science. Philosophical Transactions of the Royal Society B 373:20170051. [arFO]

Heyes, C. M. (1994) Social learning in animals: Categories and mechanisms. Biological Reviews 69:207-31. [aFO]

Heyes, C. M. (2012) What's social about social learning? Journal of Comparative Psychology 126:193-202. [aFO]

Heyes, C. M. \& Galef, B. G. Jr., eds. (1996) Social learning and the roots of culture. Academic Press. [MJO]

Ho, S.-Y., Tong, E. M. W. \& Jia, L. (2016) Authentic and hubristic pride: Differential effects on delay of gratification. Emotion (Washington, D.C.) 16:1147-56. [JLT]

Hodges, J. R., Bozeat, S., Lambon Ralph, M. A., Patterson, K. \& Spatt, J. (2000) The role of knowledge in object use: Evidence from semantic dementia. Brain 123:1913-25. [arFO]

Hodges, J. R., Spatt, J. \& Patterson, K. (1999) 'What' and 'how': Evidence for the dissociation of object knowledge and mechanical problem-solving skills in the human brain. Proceeding of the National Academy of Sciences USA 96:9444-48. [aFO]

Holzhaider, J. C., Hunt, G. R., Campbell, V. M. \& Gray, R. D. (2008) Do wild New Caledonian crows (Corvus moneduloides) attend to the functional properties of their tools? Animal Cognition 11:243-54. [CR]

Holzhaider, J. C., Hunt, G. R. \& Gray, R. D. (2010a) Social learning in New Caledonian crows. Learning \& Behavior 38:206-19. [CR]

Holzhaider, J. C., Hunt, G. R. \& Gray, R. D. (2010b) The development of pandanus tool manufacture in wild New Caledonian crows. Behaviour 147:553-86. [AHT]

Holzhaider, J. C., Sibley, M. D., Taylor, A. H., Singh, P. J., Gray, R. D. \& Hunt, G. R. (2011) The social structure of New Caledonian crows. Animal Behaviour 81(1):8392. [AHT]

Hopkins, W. D., Meguerditchian, A., Coulon, O., Misiura, M., Pope, S., Mareno, M. C. \& Schapiro, S. J. (2017) Motor skill for tool-use is associated with asymmetries in Broca's area and the motor hand area of the precentral gyrus in chimpanzees (Pan troglodytes). Behavioural Brain Research 318:71-81. Available at: http://doi.org/10.1016/j. bbr.2016.10.048. [TG]

Hopper, L. M., Lambeth, S. P., Schapiro, S. J. \& Whiten, A. (2008) Observational learning in chimpanzees and children studied through 'ghost' conditions. Proceedings of the Royal Society B 275(1636):835-40. [AM-R]

Hoppitt, W. \& Laland, K. N. (2013) Social learning: An introduction to mechanisms, methods, and models. Princeton University Press. [aFO, MJO]

Horner, V., Proctor, D., Bonnie, K. E., Whiten, A. \& de Waal, F. B. M. (2010) Prestige affects cultural learning in chimpanzees. PLoS One 5:e10625. [aFO]

Horner, V. \& Whiten, A. (2005) Causal knowledge and imitation/emulation switching in chimpanzees (Pan troglodytes) and children (Homo sapiens). Animal Cognition 8(3):164-81. [aFO, AM-R, AW]

Horner, V., Whiten, A., Flynn, E. \& de Waal, F. B. M. (2006) Faithful replication of foraging techniques along cultural transmission chains by chimpanzees and children. Proceedings of the National Academy of Sciences USA 103:13878-83. [arFO, AM-R]

Houkes, W. \& Vermaas, P. (2010) Technical functions: On the use and design of artefacts. Springer. [WH]

Hubert, H. \& Mauss, M. (1902) Esquisse d'une théorie générale de la magie. L’Année Sociologique 7:1-146. [aFO]

Humphrey, N. K. (1976) The social function of intellect. In: Growing points in ethology, eds. P. P. Bateson and R. Hinde, pp. 303-317. Cambridge University Press. [CB]

Humphreys, G. (2001) Objects, affordances... actions!. The Psychologist 14:408-12. [arFO]

Hunt, G. R. (1996) Manufacture and use of hook-tools by New Caledonian crows. Nature 379(6562):249-51. [aFO, AHT, CR]

Hunt, G. R. (2000) Human-like, population-level specialization in the manufacture of pandanus tools by New Caledonian crows Corvus moneduloides. Proceedings of the Royal Society of London. Series B: Biological Sciences 267(1441):403-13. [AHT]

Hunt, G. R., Holzhaider, J. C. \& Gray, R. D. (2012) Prolonged parental feeding in tool using New Caledonian crows. Ethology 118:423-30. [CR]

Hunt, G., Rutledge, R. \& Gray, R. (2006) The right tool for the job: What strategies do wild New Caledonian crows use? Animal Cognition 9:307-16. [CR]

Hunt, G. R. \& Gray, R. D. (2003) Diversification and cumulative evolution in New Caledonian crow tool manufacture. Proceedings of the Royal Society of London B: Biological Sciences 270(1517):867-74. [aFO, AHT, CR]
Hunt, G. R. \& Gray, R. D. (2004) The crafting of hook tools by wild New Caledonian crows. Proceedings of the Royal Society of London B: Biological Sciences 271:S88-90. [AHT]

Hunt, G. R. \& Uomini, N. (2016) A complex adaptive system may be essential for cumulative modifications in tool design. Japanese Journal of Animal Psychology 66:141-59. [CR]

Ingold, T. (1993) Tool-use, sociality and intelligence. In: Tools, language and cognition in human evolution, eds. K. R. Gibson \& T. Ingold, pp. 429-45. Cambridge University Press. [aFO]

Ingold, T. (1998) Comments on Boesch and Tomasello 'Chimpanzee and human cultures'. Current Anthropology 39:606-607. [aFO]

Inoue-Nakamura, N. \& Matsuzawa, T. (1997) Development of stone tool use by wild chimpanzees (Pan troglodytes). Journal of Comparative Psychology 111:159-73. [JJL]

Irish, M., Hodges, J. R. \& Piguet, O. (2014) Right anterior lobe dysfunction underlies theory of mind impairments in semantic dementia. Brain 137:1241-53. [rFO]

Isaacson, W. (2011) Steve Jobs. Simon \& Schuster. [BJC]

Jack, A. I., Dawson, A. J., Begany, K. L., Leckie, R. L., Barry, K. P., Ciccia, A. H. \& Snyder, A. Z. (2013) fMRI reveals reciprocal inhibition between social and physical cognitive domains. NeuroImage 66:385-401. Available at: https://doi.org/10.1016/j. neuroimage.2012.10.061. [BJC]

Jack, A. I. \& Gabriel, J. (2013) Individual and gender differences demonstrate a trade-off between empathetic concern and physical reasoning abilities. Department of Cognitive Science, Case Western University. Available at: http://tonyjack.org/files/2013\%20April \%20Poster\%20IRI-EC\%20vs.\%20IPT.pdf. [BJC]

Jacobs, I. F., von Bayern, A., Martin-Ordas, G., Rat-Fischer, L. \& Osvath, M. (2015) Corvids create novel causal interventions after all. Proceedings of the Royal Society B: Biological Sciences 282(1806):20142504. [AHT, CR]

James, W. (2007) The principles of psychology. Cosimo Classics (Original work published in 1890). [aFO]

Jarry, C., Osiurak, F., Delafuys, D., Chauviré, V., Etcharry-Bouyx, F. \& Le Gall, D. (2013) Apraxia of tool use: More evidence for the technical reasoning hypothesis. Cortex 49:2322-33. [aFO]

Jelbert, S. A., Hosking, R. J., Taylor, A. H. \& Gray, R. D. (2018) Mental template matching is a potential cultural transmission mechanism for New Caledonian crow tool manufacturing traditions. Scientific Reports 8:8956. [AHT, CR, rFO]

Jelbert, S. A., Miller, R., Schiestl, M., Boeckle, M., Cheke, L. G., Gray, R. D., ... \& Clayton, N. S. (2019) New Caledonian crows infer the weight of objects from observing their movements in a breeze. Proceedings of the Royal Society B: Biological Sciences 286 (1894):20182332. [AHT]

Jelbert, S. A., Singh, P. J., Gray, R. D. \& Taylor, A. H. (2015a) New Caledonian crows rapidly solve a collaborative problem without cooperative cognition. PLoS ONE 10(8). [AHT]

Jelbert, S. A., Taylor, A. H. \& Gray, R. D. (2015b) Reasoning by exclusion in New Caledonian crows (Corvus moneduloides) cannot be explained by avoidance of empty containers. Journal of Comparative Psychology 129(3):283. [AHT]

Jipson, J. L., Gülgöz, S. \& Gelman, S. A. (2016) Parent-child conversations regarding the ontological status of a robotic dog. Cognitive Development 39:21-35. Available at: https://doi.org/10.1016/j.cogdev.2016.03.001. [ASH]

Kahrs, B. A., Jung, W. P. \& Lockman, J. J. (2014) When does tool use become distinctively human? Hammering in young children. Child Development 85:1050-1061. [JJL]

Kelemen, D. \& Rosset, E. (2009) The human function compunction: Teleological explanation in adults. Cognition 111(1):138-43. [MH]

Keller, C. \& Keller, J. (1996) Cognition and tool use: The blacksmith at work. Cambridge University Press. [aFO]

Kemp, M. \& Mesoudi, A. (2014) An experimental demonstration of the effect of group size on cultural accumulation. Evolution and Human Behavior 35:285-90. [aFO]

Kendal, R. L. (2019) Explaining human technology. Nature Human Behaviour 3:422-23. [aFO]

Kendal, R. L., Boogert, N. J., Rendell, L., Laland, K. N., Webster, M. \& Jones, P. L. (2018) Social learning strategies: Bridge-building between fields. Trends in Cognitive Sciences 22:651-65. Available at: https://doi.org/10.1016/j.tics.2018.04.003. [aFO, AT]

Kendal, R. L., Coolen, I., van Bergen, Y. \& Laland, K. N. (2005) Trade-offs in the adaptive use of social and asocial learning. Advances in the Study of Behavior 35:333-79. [aFO]

Kendal, R., Hopper, L. M., Whiten, A., Brosnan, S. F., Lambeth, S. P., Schapiro, S. J. \& Hoppitt, W. (2015) Chimpanzees copy dominant and knowledgeable individuals: Implications for cultural diversity. Evolution and Human Behavior 36:65-72. [aFO]

Kenward, B., Rutz, C., Weir, A. A. S. \& Kacelnik, A. (2006) Development of tool use in New Caledonian crows: Inherited action patterns and social influences. Animal Behaviour 72:1329-43. [CR]

Kenward, B., Weir, A. A. S., Rutz, C. \& Kacelnik, A. (2005) Tool manufacture by naive juvenile crows. Nature 433(7022):121. [AHT, CR]

Kenward, B., Schloegl, C., Rutz, C., Weir, A. A. S., Bugnyar, T. \& Kacelnik, A. (2011) On the evolutionary and ontogenetic origins of tool-oriented behaviour in New Caledonian crows (Corvus moneduloides). Biological Journal of the Linnean Society 102:870-77. [JJL] 
Kern, A. \& Moll, H. (2017) On the transformative character of collective intentionality and the uniqueness of the human. Philosophical Psychology 30(3):319-37. doi: 10.1080/09515089.2017.1295648. [HM]

Kirby, S., Cornish, H. \& Smith, K. (2008) Cumulative cultural evolution in the laboratory: An experimental approach to the origins of structure in human language. Proceeding of the National Academy of Sciences of the United States of America 105(31):10681-86. [EM]

Kirchhoff, M. D. \& Robertson, I. (2018) Enactivism and predictive processing: A non-representational view. Philosophical Explorations 21(2):264-81. [IR]

Kline, M. A. (2015) How to learn about teaching: An evolutionary framework for the study of teaching behavior in humans and other animals. Behavioral and Brain Sciences 38:1-70. Available at: https://doi.org/10.1017/S0140525X14000090. [aFO, PG]

Knaebe, B., Taylor, A. H., Elliffe, D. M. \& Gray, R. D. (2017) New Caledonian crows show behavioural flexibility when manufacturing their tools. Behaviour 154:65-91. [CR]

Knaebe, B., Taylor, A. H., Miller, R. \& Gray, R. D. (2015) New Caledonian crows (Corvus moneduloides) attend to barb presence during pandanus tool manufacture and use. Behaviour 152:2107-2125. [CR]

Koenig, M. A. \& Woodward, A. L. (2010) 24-Month-olds' sensitivity to the prior inaccuracy of the source: Possible mechanisms. Developmental Psychology 46:815-26. [JLT]

Köhler, W. (1959) The mentality of apes. Vintage Books. [aFO]

Koops, K., Visalberghi, E. \& van Schaik, C. P. (2014) The ecology of primate material culture. Biology Letters 10:20140508. [aFO]

Kuczaj, S. A. (2017) Animal creativity and innovation. In: APA handbooks in psychology ${ }^{\varpi}$. APA handbook of comparative psychology: Perception, learning, and cognition. ed. J. Call, G. M. Burghardt, I. M. Pepperberg, C. T. Snowdon \& T. Zentall, pp. 627-41. American Psychological Association. https://doi.org/10.1037/0000012-028. [AW]

Kurkul, K., Castine, E., Leech, K. \& Corriveau, K. H. (under revised review) 'How does a switch work?' The role of mechanistic language in children's learning from parents Submitted to Journal of Applied Developmental Psychology. [ASH]

Kurkul, K. E. \& Corriveau, K. H. (2018) Question, explanation, follow-up: A mechanism for learning from others? Child Development 89(1):280-94. Available at: https://doi org/10.1111/cdev.12726. [ASH]

Kyaga, S., Lichtenstein, P., Boman, M., Hultman, C., Långström, N. \& Landén, M. (2011) Creativity and mental disorder: Family study of 300000 people with severe mental disorder. The British Journal of Psychiatry: The Journal of Mental Science 199:373-79. Available at: https://doi.org/10.1192/bjp.bp.110.085316. [BJC]

Laland, K. (2004) Social learning strategies. Learning and Behavior 32:4-14. [aFO]

Laland, K. N. (2017a) Darwin's unfinished symphony: How culture made the human mind. Princeton University Press. [EM]

Laland, K. N. (2017b) The origins of language in teaching. Psychonomic Bulletin and Review 24:225-31. Available at: https://doi.org/10.3758/s13423-016-1077-7. [AT]

Laland, K. N., Odling-Smee, J. \& Myles, S. (2010) How culture shaped the human genome: Bringing genetics and the human sciences together. Nature Reviews Genetics 11:137-48. [rFO]

Lambourne, K. \& Tomporowski, P. (2010) The effect of exercise-induced arousal on cog nitive task performance: A meta-regression analysis. Brain Research 1341:12-24. [CB]

Lancy, D. F. (2017) Raising children: Surprising insights from other cultures. Cambridge University Press. [JJL]

Lederman, S. J. \& Klatzky, R. L. (1987) Hand movements: A window into haptic object recognition. Cognitive Psychology 19:342-68. [JJL]

Lee, C. C., Czaja, S. J., Moxley, J. H., Sharit, J., Boot, W. R., Charness, N. \& Rogers, W. A. (2019) Attitudes toward computers across adulthood from 1994 to 2013. The Gerontologist 59(1):22-33. [JFB]

Leech, K. A., Haber, A. S., Jalkh, Y. \& Corriveau, K. H. (in press) Embedding explanations into storybooks impacts children's scientific discourse and learning. Frontiers in Psychology 11:1016. DOI:10.3389/fpsyg.2020.01016. [ASH]

Leeuwen, E., Mulenga, I. \& Chidester, D. (2014) Early social deprivation negatively affects social skill acquisition in chimpanzees (Pan troglodytes). Animal Cognition 17:407-14. [CB]

Legare, C. H. \& Nielsen, M. (2015) Imitation and innovation: The dual engines of cultural learning. Trends in Cognitive Sciences 19(11):688-99. Available at: https:// doi.org/10.1016/j.tics.2015.08.005. [aFO, BR]

Lesourd, M., Baumard, J., Jarry, C., Etcharry-Bouyx, F., Belliard, S., Moreaud, O., Croisile, B., Chauviré, V., Granjon, M., Le Gall, D. \& Osiurak, F. (2016) Mechanical problemsolving strategies in Alzheimer's disease and semantic dementia. Neuropsychology 30:612-23. [arFO]

Lesourd, M., Baumard, J., Jarry, C., Le Gall, D. \& Osiurak, F. (2017) A cognitive-based mode of tool use in normal aging. Aging, Neuropsychology, and Cognition 24:363-86. [aFO]

Levine, S. C., Suriyakham, L. W., Rowe, M. L., Huttenlocher, J. \& Gunderson, E. A. (2010) What counts in the development of young children's number knowledge? Developmental Psychology 46(5):1309-19. Available at: doi: 10.1037/a0019671. [ASH]

Lewis, H. M. \& Laland, K. N. (2012) Transmission fidelity is the key to the build-up of cumulative culture. Philosophical Transactions of the Royal Society B 367:2171-80. [aFO]

Liu, Q., Simpson, K., Izar, P., Ottoni, E., Visalberghi, E. \& Fragaszy, D. (2009) Kinematics and energetics of nut-cracking in wild capuchin monkeys (Cebus libidinosus) in Piauí, Brazil. American Journal of Physical Anthropology 188:210-20. [aFO]
Liu, S., Brooks, N. B. \& Spelke, E. S. (2019) Origins of the concepts cause, cost, and goal in prereaching infants. Proceedings of the National Academy of Sciences 116(36):1774752. $[\mathrm{MH}]$

Lockman, J. J. (2000) A perception-action perspective on tool use development. Child Development 71:137-44. [JJL]

Logan, C. J., Breen, A. J., Taylor, A. H., Gray, R. D. \& Hoppitt, W. J. S. E. (2016) How New Caledonian crows solve novel foraging problems and what it means for cumulative culture. Learning \& Behavior 44(1):18-28. [AHT, CR, rFO]

Lombard, M. \& Gärdenfors, P. (2017) Tracking the evolution of causal cognition in humans. Journal of Anthropological Sciences 95:1-18. [PG]

Lombard, M., Högberg, A. \& Haidle, M. N. (2019) Cognition: From Capuchin rock pounding to Lomekwian flake production. Cambridge Archaeological Journal 29(2):201-31. [PG]

Lombrozo, T., Bonawitz, E. B. \& Scalise, N. R. (2018) Young children's learning and generalization of teleological and mechanistic explanations. Journal of Cognition and Development 19(2):220-32. Available at: https://doi.org/10.1080/15248372.2018. 1427099. [ASH]

Lotem, A., Halpern, J. Y., Edelman, S. \& Kolodny, O. (2017) The evolution of cognitive mechanisms in response to cultural innovations. Proceedings of the National Academy of Science USA 114:7915-22. [aFO]

Lucas, A. J., Burdett, E. R., Burgess, V., McGuigan, N., Wood, L. A., Harris, P. L. \& Whiten, A. (2017) Children's selective copying of their mother versus an expert. Child Development 88:2026-42. [ERRB]

Luncz, L., Mundry, R. \& Boesch, C. (2012) Evidence for cultural differences between neighboring chimpanzee communities. Current Biology 22:922-26. [CB]

Luncz, L. V., Falótico, T., Pascual-Garrido, A., Corat, C., Monsley, H. \& Haslam, M. (2016) Wild capuchin monkeys adjust stone tools according to changing nut properties. Scientific Reports 6:33089. [aFO]

Lyons, D. E., Damrosch, D. H., Lin, J. K., Macris, D. M. \& Keil, F. C. (2011) The scope and limits of overimitation in the transmission of artefact culture. Philosophical Transactions of the Royal Society B 366:1158-67. [aFO]

Lyons, D. E., Young, A. G. \& Keil, F. C. (2007) The hidden structure of overimitation. Proceedings of the National Academy of Sciences USA 104(50):19751-56. doi: 10.1073/pnas.0704452104. [aFO, HM]

MacDonald, K. (2007) Cross-cultural comparison of learning in human hunting. Human Nature 18:386-402. [aFO]

Mannu, M. \& Ottoni, E. B. (2009) The enhanced tool-kit of two groups of wild bearded capuchin monkeys in the Caatinga: Tool making, associative use, and secondary tools. American Journal of Primatology 71:242-51. [aFO]

Maravita, A. \& Iriki, A. (2004) Tools for the body (schema). Trends in Cognitive Sciences 8:79-86. doi: 10.1016/j.tics.2003.12.008. [PG]

Marshall-Pescini, S. \& Whiten, A. (2008) Chimpanzees (Pan troglodytes) and the question of cumulative culture: An experimental approach. Animal Cognition 11:449-56. [AW]

Martens, J. P. \& Tracy, J. L. (2013) The emotional origins of a social learning bias: Does the pride expression cue copying? Social Psychological and Personality Science 4:49299. [JLT]

Martin, M., Beume, L., Kümmerer, D., Schmidt, C. S. M., Bormann, T., Dressing, A., Ludwig, V. M., Umarova, R. M., Mader, I., Rijntjes, M., Kaller, C. P. \& Weiller, C. (2016) Differential roles of ventral and dorsal streams for conceptual and production-related components of tool use in acute stroke patients. Cerebral Cortex 26:3754-71. [aFO]

Martin-Ordas, G., Call, J. \& Colmenares, F. (2008) Tubes, tables and traps: Great apes solve two functionally equivalent trap tasks but show no evidence of transfer across tasks. Animal Cognition 11:423-30. [aFO]

McCloskey, M. (1983) Intuitive physics. Scientific American 248:395-400. [aFO]

McGuigan, N. (2012) The role of transmission biases in the cultural diffusion of irrelevant actions. Journal of Comparative Psychology 126:150-60. [aFO]

McGuigan, N., Burdett, E., Burgess, V., Dean, L., Lucas, A., Vale, G. \& Whiten, A. (2017) Innovation and social transmission in experimental micro-societies: Exploring the scope of cumulative culture in young children. Philosophical Transactions of the Royal Society B: Biological Sciences 372:1735. [ERRB]

McGuigan, N., Makinson, J. \& Whiten, A. (2011) From overimitation to super-copying: Adults imitate irrelevant aspects of tool use with higher fidelity than young children. British Journal of Psychology 102:1-18. [aFO]

McGuigan, N. \& Whiten, A. (2009) Emulation and 'overemulation' in the social learning of causally opaque versus causally transparent tool use by 23- and 30-month-old children. Journal of Experimental Child Psychology 104:367-81. [aFO]

McGuigan, N., Whiten, A., Flynn, E. F. \& Horner, V. (2007) Imitation of causally opaque versus causally transparent tool use by 3 - and 5-year-old children. Cognitive Development 22(3):353-64. doi: 10.1016/j.cogdev.2007.01.001. [aFO, HM]

Mead, M. (1934) Mind, self, and society. University of Chicago Press. [aFO]

Meltzoff, A. N., Waismeyer, A. \& Gopnik, A. (2012) Learning about causes from people: Observational causal learning in 24-month-old infants. Developmental Psychology 48:1215-28. [MD]

Menary, R. (2010) The extended mind. MIT Press. [CK] 
Mesoudi, A. (2008) An experimental simulation of the 'copy-successful-individuals' cultural learning strategy: Adaptive landscapes, producer-scrounger dynamics, and informational access costs. Evolution and Human Behavior 29:350-63. [MJO]

Mesoudi, A. (2010) The experimental study of cultural innovation. In: Innovation in cultural systems: Contributions from evolutionary anthropology, eds. M. J. O’Brien \& S. J. Shennan, pp. 175-91. MIT Press. [MJO]

Mesoudi, A. (2011) Variable cultural acquisition costs constrain cumulative cultural evolution. PLoS One 6:e18239. [rFO]

Mesoudi, A. (2016) Cultural evolution: A review of theory, findings and controversies. Evolutionary Biology 43(4):481-97. [EM]

Mesoudi, A., Chang, L., Dall, S. R. X. \& Thornton, A. (2016) The evolution of individual and cultural variation in social learning. Trends in Ecology and Evolution 31(3):21525. [EM, MJO]

Mesoudi, A. \& O'Brien, M. J. (2008) The cultural transmission of Great Basin projectilepoint technology I: An experimental simulation. American Antiquity 73:3-28. [MJO]

Mesoudi, A. \& Thornton, A. (2018) What is cumulative cultural evolution? Proceedings of the Royal Society B 285:20180712. Available at: https://doi.org/10.1098/rspb.2018.0712. [aFO, AT, CR, MJO]

Mesoudi, A. \& Whiten, A. (2008) The multiple roles of cultural transmission experiments in understanding human cultural evolution. Philosophical Transactions of the Royal Society B 363:3489-3501. [aFO, MJO]

Middleton, L., Mitnitski, A., Fallah, N., Kirkland, S. \& Rockwood, K. (2008) Changes in cognition and mortality in relation to exercise in late life: A population based study. PLoS ONE 3:e3124. [CB]

Mithen, S. (1996a) The prehistory of the mind. Thames and Hudson. [AW]

Mithen, S. (1996b) The prehistory of the mind: A search for the origins of art, religion and science. Phoenix. [LG]

Miton, H. \& Charbonneau, M. (2018) Cumulative culture in the laboratory: Methodological and theoretical challenges. Proceedings of the Royal Society B: Biological Sciences 285(1879):20180677. [MD]

Moll, H. (2018) The transformative cultural intelligence hypothesis: Evidence from young children's problem-solving. Review of Philosophy and Psychology 9(1):161-75. doi: 10.1007/s13164-017-0342-7. [HM, rFO]

Moll, H. \& Tomasello, M. (2007) Cooperation and human cognition: The Vygotskian intelligence hypothesis. Philosophical Transactions of the Royal Society B 362:639-48. [aFO]

Morgan, T. J. H. (2016) Testing the cognitive and cultural niche theories of human evolution. Current Anthropology 57(3):370-77. [EM]

Morgan, T. J. H., Rendell, L. E., Ehn, M., Hoppitt, W. \& Laland, K. N. (2012) The evolutionary basis of human social learning. Proceedings of the Royal Society B 279:653-62. [aFO]

Morgan, T. J. H., Uomini, N. T., Rendell, L. E., Chouinard-Thuly, L., Street, S. E., Lewis, H. M., Cross, C. P., Evans, C., Kearney, R., de la Torre, I., Whiten, A. \& Laland, K. N. (2015) Experimental evidence for the co-evolution of hominin tool-making teaching and language. Nature Communications 6:1-8. Available at: https://doi.org/10.1038/ ncomms7029. [aFO, AT, NC, TG]

Morin, O. (2015) How traditions live and die. Oxford University Press. [aFO]

Morin, O. (2016) Reasons to be fussy about cultural evolution. Biology \& Philosophy 31:447-58. [aFO]

Mulcahy, N. J. \& Call, J. (2006) Apes save tools for future use. Science (New York, N.Y.) 312(5776):1038-40. [MH]

Musgrave, S., Lonsdorf, E., Morgan, D., Prestipino, M., Bernstein-Kurtycz, L., Mundry, R. \& Sanz, C. (2020) Teaching varies with task complexity in wild chimpanzees. Proceedings of the National Academy of Sciences of the United States of America 117(2):969-76. doi: 10.1073/pnas.1907476116. [BJC, CB, TG]

Musgrave, S., Morgan, D., Lonsdorf, E., Mundry, R. \& Sanz, C. (2016) Tool transfers are a form of teaching among chimpanzees. Scientific Reports 6:34783. doi: 10.1038/ srep34783. [CB]

Muthukrishna, M. \& Henrich, J. (2016) Innovation in the collective brain. Philosophical Transactions of the Royal Society B: Biological Sciences 371(1690):20150192. Available at: https://doi.org/10.1098/rstb.2015.0192. [BR, EM]

Muthukrishna, M., Shulman, B. W., Vasilescu, V. \& Henrich, J. (2014) Sociality influences cultural complexity. Proceedings of the Royal Society B: Biological Sciences 281(1774):20132511. doi: 10.1098/rspb.2013.2511. [aFO, CK]

Nagell, K., Olguin, R. S. \& Tomasello, M. (1993) Processes of social learning in the tool use of chimpanzees (Pan troglodytes) and human children (Homo sapiens). Journal of Comparative Psychology 107:174-86. [aFO]

Needham, A. \& Baillargeon, R. (1993) Intuitions about support in 4.5-month-old infants. Cognition 47:121-48. [aFO]

Negri, G. A., Lunardelli, A., Reverberi, C., Gigli, G. L. \& Rumiati, R. I. (2007) Degraded semantic knowledge and accurate object use. Cortex 43:376-88. [aFO]

Neilands, P. D., Jelbert, S. A., Breen, A. J., Schiestl, M. \& Taylor, A. H. (2016) How insightful is 'insight'? New Caledonian crows do not attend to object weight during spontaneous stone dropping. PLoS ONE 11(12):e0167419. [AHT]

Nettle, D. (2006) Schizotypy and mental health amongst poets, visual artists, and mathematicians. Journal of Research in Personality 40:876-90. Available at: https://doi.org/10.1016/j.jrp.2005.09.004. [BJC]
Neves, B. B., Franz, R., Judges, R., Beermann, C. \& Baecker, R. (2019) Can digital technology enhance social connectedness among older adults? A feasibility study. Journal of Applied Gerontology 38(1):49-72. [JFB]

Nielsen, M. (2013) Young children's imitative and innovative behaviour on the floating object task. Infant and Child Development 22(6):44-52. Available at: https://doi.org/ 10.1002/icd.1765. [AT, rFO]

Nielsen, M. \& Tomaselli, K. (2010) Over-imitation in Kalahari Bushman children and the origins of human cultural cognition. Psychological Science 21:729-36. [aFO]

Noble, K., Houston, S., Brito, N., Bartsch, H., Kan, E., Kuperman, J., Akshoomoff, N., Amaral, D., Bloss, C., Libiger, O., Schork, N., Murray, S., Casey, B., Chang, L., Ernst, T., Frazier, J., Gruen, J., Kennedy, D., Van Zijl, P., Mostofsky, S., Kaufmann, W., Kenet, T., Dale, A., Jernigan, T. \& Sowell, E. (2015) Family income, parental education and brain structure in children and adolescents. Nature Neuroscience 18:77380. [CB]

Normand, E., Ban, S. \& Boesch, C. (2009) Forest chimpanzees (Pan troglodytes verus) remember the location of numerous fruit trees. Animal Cognition 12:797-807. [CB]

Normand, E. \& Boesch, C. (2009) Sophisticated Euclidian maps in forest chimpanzees. Animal Behaviour 77:1195-201. [CB]

Oakley, B. F., Brewer, R., Bird, G. \& Catmur, C. (2016) Theory of mind is not theory of emotion: A cautionary note on the Reading the Mind in the Eyes Test. Journal of Abnormal Psychology 125(6):818. [NW]

O'Brien, M. J. (2019) More on Clovis learning: Individual-level processes aggregate to form population-level patterns. PaleoAmerica 5:157-68. [MJO]

O’Brien, M. J. \& Buchanan, B. (2017) Cultural learning and the Clovis colonization of North America. Evolutionary Anthropology 26:270-84. [MJO]

Orban, G. A. \& Caruana, F. (2014) The neural basis of human tool use. Frontiers in Psychology 5:310. [aFO]

Osherson, D. \& Smith, E. (1981) On the adequacy of prototype theory as a theory of concepts. Cognition 9:35-58. [LG]

Osiurak, F. (2014) What neuropsychology tells us about human tool use? The four constraints theory (4CT): Mechanics, space, time and effort. Neuropsychology Review 24:88-115. [arFO]

Osiurak, F. (2017) Cognitive paleoanthropology and technology: Toward a parsimonious theory (PATH). Review of General Psychology 21:292-307. [arFO]

Osiurak, F., Aubin, G., Allain, P., Jarry, C., Richard, I. \& Le Gall, D. (2008) Object utilization versus object usage: A single-case study. Neurocase 14:169-83. [arFO]

Osiurak, F. \& Badets, A. (2014) Pliers, not fingers: Tool-action effect in motor intention. Cognition 130:66-73. [aFO]

Osiurak, F. \& Badets, A. (2016) Tool use and affordance: Manipulation-based versus reasoning-based approaches. Psychological Review 123:534-68. [arFO]

Osiurak, F. \& Badets, A. (2017) Use of tools and misuse of embodied cognition. Psychological Review 124:361-68. [aFO]

Osiurak, F., Cretel, C., Duhau-Marmon, N., Fournier, I., Marignier, L., De Oliveira, E., Navarro, J. \& Reynaud, E. (in press) The pedagogue, the engineer, and the friend: From whom do we learn? Human Nature. [arFO]

Osiurak, F., De Oliveira, E., Navarro, J., Lesourd, M., Claidière, N. \& Reynaud, E. (2016) Physical intelligence does matter to cumulative technological culture. Journal of Experimental Psychology: General 145(8):941-48. [arFO, NW]

Osiurak, F., De Oliveira, E., Navarro, J. \& Reynaud, E. (2020a) The castaway island: Distinct roles of theory of mind and technical reasoning in cumulative technological culture. Journal of Experimental Psychology: General 149(1):58-66. Available at: https://doi.org/10.1037/xge0000614. [arFO, BJC]

Osiurak, F. \& Heinke, D. (2018) Looking for Intoolligence: A unified framework for the cognitive study of human tool use and technology. American Psychologist 73:169-85. [arFO]

Osiurak, F., Jarry, C., Allain, P., Aubin, G., Etcharry-Bouyx, F., Richard, I., Bernard, I. \& Le Gall, D. (2009) Unusual use of objects after unilateral brain damage: The technical reasoning model. Cortex 45:769-83. [aFO]

Osiurak, F., Jarry, C. \& Le Gall, D. (2010) Grasping the affordances, understanding the reasoning: Toward a dialectical theory of human tool use. Psychological Review 117:517-40. [arFO]

Osiurak, F., Jarry, C., Lesourd, M., Baumard, J. \& Le Gall, D. (2013) Mechanical problemsolving in left brain-damaged patients and apraxia of tool use. Neuropsychologia 51:1964-72. [aFO]

Osiurak, F., Lesourd, M., Navarro, J. \& Reynaud, E. (2020b) Technition: When tools come out of the closet. Perspectives on Psychological Science 15:880-97. [rFO]

Osiurak, F., Morgado, N., Vallet, G. T., Drot, M. \& Palluel-Germain, R. (2014) Getting a tool gives wings: Underestimation of effort for tool use. Psychological Research 78:1-9. [aFO] Osiurak, F. \& Rossetti, Y. (2017) Definition: Limb apraxia. Cortex 93:228. [rFO]

Osiurak, F., Rossetti, Y. \& Badets, A. (2017) What is an affordance? 40 years later. Neuroscience \& BioBehavioral Reviews 77:403-17. [arFO]

Overmann \& Coolidge, eds. (2019) Squeezing minds from stones: Cognitive archaeology and the evolution of the human mind. Oxford University Press. [LG]

Penn, D. C., Holyoak, K. J. \& Povinelli, D. J. (2008a) Darwin's mistake: Explaining the discontinuity between human and nonhuman minds. Behavioral and Brain Sciences 31(2):109-78. [LG] 
Penn, D. C., Holyoak, K. J. \& Povinelli, D. J. (2008b) Darwin's mistake: Explaining the discontinuity between human and nonhuman minds. Behavioral and Brain Sciences 31(2):109-30. [aFO, NW, LG]

Penn, D. C. \& Povinelli, D. J. (2007) Causal cognition in human and nonhuman animals A comparative, critical review. Annual Review of Psychology 58:97-118. [aFO, NW, PG]

Pew Research Center. (2018) Millennials standout for their technology use. https://www pewresearch.org/fact-tank/2018/05/02/millennials-stand-out-for-their-technology-usebut-older-generations-also-embrace-digital-life/ (accessed May 28, 2019). [JFB]

Piaget, J. (1932) The moral judgment of the child. Routledge \& Kegan Paul. [aFO]

Pike, T., Ramsey, M. \& Wilkinson, A. (2018) Environmentally induced changes to brain morphology predict cognitive performance. Philosophical Transactions of the Royal Society B: Biological Sciences 373:20170287. [CB]

Pinker, S. (2010) The cognitive niche: Coevolution of intelligence, sociality, and language. Proceedings of the National Academy of Science of the USA 107(Suppl. 2):8993-99. [aFO, IR, PG]

Plancher, G. \& Barrouillet, P. (2020) On some of the main criticisms of the modal model: Reappraisal from a TBRS perspective. Memory \& Cognition 48:455-68. [rFO]

Povinelli, D. (2000) Folk physics for apes: The chimpanzee's theory of how the world works. Oxford University Press. [CB]

Povinelli, D. (2012) World without weight: Perspectives on an Alien mind. Oxford University Press. [CB]

Povinelli, D. J. \& Frey, S. H. (2016) Constraints on the exploitation of the functional properties of objects in expert tool-using chimpanzees (Pan troglodytes). Cortex 82:11-23. [aFO]

Premack, D. (1988) 'Does the chimpanzee have a theory of mind?' revisited. In: Machiavellian intelligence: Social expertise and the evolution of intellect in monkeys, apes, and humans, eds. R. Byrne \& A. Whiten, pp. 160-79. Oxford University Press. [aFO]

Premack, D. \& Premack, A. (1983) The mind of an ape. Norton. [CB]

Price, E. E., Lambeth, S. P., Schapiro, S. J. \& Whiten, A. (2009) A potent effect of observational learning on chimpanzee tool construction. Philosophical Transactions of the Royal Society B 276:3377-83. [aFO]

Putt, S. S., Woods, A. D. \& Franciscus, R. D. (2014) The role of verbal interaction during experimental bifacial stone tool manufacture. Lithic Technology 39:96-112. [aFO]

Quesque, F. \& Rossetti, Y. (2020) What do theory-of-mind tasks actually measure? Theory and practice. Perspectives on Psychological Science 15:384-96. [rFO]

Rachwani, J., Tamis-LeMonda, C. S., Lockman, J. J., Karasik, L. B. \& Adolph, K. E. (2020) Learning the designed actions of everyday objects. Journal of Experimental Psychology: General 149:67-78. [JJL]

Ramani, G. B., Rowe, M. L., Eason, S. H. \& Leech, K. A. (2015) Math talk during informal learning activities in head start families. Cognitive Development 35:15-33. doi: 10 1016/j.cogdev.2014.11.002. [ASH]

Ramsey, G., Bastian, M. L. \& van Schaik, C. (2007) Animal innovation defined and operationalized. Behavioral and Brain Sciences 30:393-437. [aFO, BR]

Reader, S. M. \& Laland, K. N. (2002) Social intelligence, innovation, and enhanced brain size in primates. Proceedings of the National Academy of Science USA 99:4436-41. [aFO]

Reindle, E., Apperly, I. A., Beck, S. R. \& Tennie, C. (2017) Young children copy cumulative technological design in the absence of action information. Scientific Reports 7:1788. [aFO, ERRB]

Reindle, E. \& Tennie, C. (2018) Young children fail to generate an additive ratchet effect in an open-ended construction task. PLoS ONE 13:e0197828. [aFO, ERRB]

Remigereau, C., Roy, A., Costini, O., Osiurak, F., Jarry, C. \& Le Gall, D. (2016) Involvement of technical reasoning more than functional knowledge in development of tool use in childhood. Frontiers in Psychology 7:1625. [aFO]

Rendell, L., Boyd, R., Cownden, D., Enquist, M., Eriksson, K., Feldman, M. W., Fogarty, L., Ghirlanda, S., Lillicrap, T. \& Laland, K. N. (2010) Why copy others? Insights from the social learning strategies tournament. Science (New York, N.Y.) 328(5975):208-13. doi: 10.1126/science.1184719. [aFO, NC]

Resende, B. D., Ottoni, E. B. \& Fragaszy, D. M. (2008) Ontogeny of manipulative behavior and nut-cracking in young tufted capuchin monkeys (Cebus apella): A perception-action perspective. Developmental Science 11:828-40. [JJL]

Reynaud, E., Lesourd, M., Navarro, J. \& Osiurak, F. (2016) On the neurocognitive origins of human tool use. A critical review of neuroimaging data. Neuroscience \& BioBehavioral Reviews 64:421-37. [aFO]

Reynaud, E., Navarro, J., Lesourd, M. \& Osiurak, F. (2019) To watch is to work: A critical review of neuroimaging data on Tool-use Observation Network (ToON). Neuropsychology Review 29:484-97. [arFO]

Reynolds, P. C. (1993) The complementation theory of language and tool use. In: Tools, language and cognition in human evolution, eds. K. R. Gibson \& T. Ingold, pp. 405-29. Cambridge University Press. [aFO]

Richerson, P. J. \& Boyd, R. (2005) Not by genes alone: How culture transformed human evolution. University of Chicago Press. [aFO, NC, TG]

Riede, F., Johannsen, N. N., Högberg, A., Nowell, A. \& Lombard, M. (2018) The role of play objects and object play in human cognitive evolution and innovation. Evolutionary Anthropology 27:46-59. [PG]
Rochat, M. J., Serra, E., Fadiga, L. \& Gallese, V. (2008) The evolution of social cognition: Goal familiarity shapes monkeys' action understanding. Current Biology 18(3):227-32. $[\mathrm{MH}]$

Roelfsema, M. T., Hoekstra, R. A., Allison, C., Wheelwright, S., Brayne, C., Matthews, F. E., et al. (2012) Are autism spectrum conditions more prevalent in an informationtechnology region? A school-based study of three regions in the Netherlands. Journal of Autism and Developmental Disorders 42:734-39. Available at: https://doi. org/10.1007/s10803-011-1302-1. [BJC]

Rogers, A. R. (1988) Does biology constrain culture? American Anthropologist 90:819-31. [aFO]

Rogers, E. M. (1995) Diffusion of innovations. Free Press. [aFO]

Ronfard, S., Zambrana, I. M., Hermansen, T. K. \& Kelemen, D. (2018) Question-asking in childhood: A review of the literature and a framework for understanding its development. Developmental Review 49:101-20. Available at: https://doi.org/10.1016/j.dr.2018. 05.002. [ASH]

Rose, D. \& Nichols, S. (2019) Teleological essentialism. Cognitive Science 43(4):e12725. [MH]

Rothi, L. J. G., Ochipa, C. \& Heilman, K. M. (1991) A cognitive neuropsychological model of limb praxis. Cognitive Neuropsychology 8:443-58. [aFO]

Rowe, C. \& Healy, S. (2014) Measuring variation in cognition. Behavioral Ecology 25:1287-92. [CB]

Russell, J., Lyn, H., Schaeffer, J. \& Hopkins, W. (2011) The role of socio-communicative rearing environment in the development of social and physical cognition in apes. Developmental Science 14:1459-70. [CB]

Rutherford, M. D. \& Subiaul, F. (2016) Children with autism spectrum disorder have an exceptional explanatory drive. Autism 20(6):744-53. Available at: https://doi.org/10. 1177/1362361315605973. [BJC]

Rutz, C. (unpublished manuscript) Tool innovation in crows. Cambridge University Press. [CR]

Rutz, C., Burns, Z. T., James, R., Ismar, S., Burt, J., Otis, B., Bowen, J. \& St Clair, J. J. H. (2012) Automated mapping of social networks in wild birds. Current Biology 22: R669-71. [CR]

Rutz, C., Hunt, G. R. \& St Clair, J. J. H. (2018) Corvid technologies: How do New Caledonian crows get their tool designs? Current Biology 28:R1109-11. [CR]

Rutz, C., Klump, B. C., Komarczyk, L., Leighton, R., Kramer, J., Wischnewski, S., Sugasawa, S., Morrissey, M. B., James, R., St Clair, J. J. H., Switzer, R. A. \& Masuda, B. M. (2016a) Discovery of species-wide tool use in the Hawaiian crow. Nature 537:403-407. [CR]

Rutz, C. \& St Clair, J. J. H. (2012) The evolutionary origins and ecological context of tool use in New Caledonian crows. Behavioural Processes 89:153-65. [CR]

Rutz, C., Sugasawa, S., van der Wal, J. E. M., Klump, B. C. \& St Clair, J. J. H. (2016b) Tool bending in New Caledonian crows. Royal Society Open Science 3:160439. [CR]

Salazar-Lopez, E., Schwaiger, B. J. \& Hermsdörfer, J. (2016) Lesion correlates of impairments in actual tool use following unilateral brain damage. Neuropsychologia 84:16780. $[\mathrm{aFO}]$

Saldana, C., Fagot, J., Kirby, S., Smith, K. \& Claidière, N. (2019) High-fidelity copying is not necessarily the key to cumulative cultural evolution: A study in monkeys and children. Proceedings of the Royal Society B: Biological Sciences 286:20190729. Available at: https://doi.org/10.1098/rspb.2019.0729. [AT]

Sallet, J., Mars, R., Noonan, M., Andersson, J., O’Reilly, J., Jbabdi, S., Croxson, P., Jenkinson, M., Miller, K. \& Rushworth, M. (2011) Social network size affects neural circuits in macaques. Science (New York, N.Y.) 334:697-700. [CB]

Salvanes, A., Moberg, O., Ebbesson, L. O. E., Nilsen, T., Jensen, K. \& Braithwaite, V. (2013) Environmental enrichment promotes neural plasticity and cognitive ability in fish. Proceedings of the Royal Society B: Biological Sciences 280:20131331. [CB]

Sanz, C. M., Call, J. \& Morgan, D. (2009) Design complexity in termite-fishing tools of chimpanzees (Pan troglodytes). Biology Letters 5:293-96. [CB, TG]

Sanz, C. \& Morgan, D. (2007) Chimpanzee tool technology in the Goualougo Triangle, Republic of Congo. Journal of Human Evolution 52:420-33. [CB]

Sanz, C. \& Morgan, D. (2009) Flexible and persistent tool-using strategies in honeygathering by wild chimpanzees. International Journal of Primatology 30:411-27. [CB]

Sanz, C. M. \& Morgan, D. B. (2013) Ecological and social correlates of chimpanzee tool use. Philosophical Transactions of the Royal Society B 368:20120416. [aFO]

Sanz, C., Morgan, D. \& Gulick, S. (2004) New insights into chimpanzees, tools, and termites from the Congo Basin. American Naturalist 164:567-81. [CB]

Sasaki, T. \& Biro, D. (2017) Cumulative culture can emerge from collective intelligence in animal groups. Nature Communications 8(1):15049. doi: 10.1038/ncomms15049. [AT, $\mathrm{NC}]$

Schillinger, K., Mesoudi, A. \& Lycett, S. (2015) The impact of imitative versus emulative learning mechanisms on artifactual variation: Implications for the evolution of material culture. Evolution and Human Behavior 36:446-55. [aFO]

Schillinger, K., Mesoudi, A. \& Lycett, S. J. (2014) Copying error and the cultural evolution of 'additive' vs. 'reductive' material traditions: An experimental assessment. American Antiquity 79:128-43. [MJO]

Schrauf, C., Huber, L. \& Visalberghi, E. (2008) Do capuchin monkeys use weight to select hammer tools? Animal Cognition 11:413-22. [aFO] 
Scotney, V. S., Schwartz, J., Carbert, N., Saab, A. \& Gabora, L. (2020) The form of a 'halfbaked' creative idea: Empirical explorations into the structure of ill-defined mental representations. Acta Psychologica 203:102981. [LG]

Seed, A. \& Mayer, C. (2017) Problem-solving. In: APA handbook of comparative psychology vol. 2: Perception, learning and cognition. ed. J. Call, pp. 601-25. American Psychological Association. [AW]

Segall, M., Dasen, P., Berry, J. \& Poortinga, Y. (1999) Human behavior in global perspective: An introduction to cross-cultural psychology, 2nd ed. Pergamon Press. [CB]

Shariff, A. F. \& Tracy, J. L. (2009) Knowing who's boss: Implicit perceptions of status from the nonverbal expression of pride. Emotion (Washington, D.C.) 9:631-39. [JLT]

Shea, J. J. (2017) Occasional, obligatory, and habitual stone tool use in hominin evolution. Evolutionary Anthropology. Available at: https://doi.org/10.1002/evan.21547. [PG]

Shea, N., Boldt, A., Bang, D., Yeung, N., Heyes, C. \& Frith, C. D. (2014) Supra-personal cognitive control and metacognition. Trends in Cognitive Sciences 18:186-93. [aFO]

Shennan, S. J. (2008) Evolution in archaeology. Annual Review of Anthropology 37:75-91. [MJO]

Shipton, C. \& Nielsen, M. (2015) Before cumulative culture: The evolutionary origins of overimitation and shared intentionality. Human Nature 26:33145. [aFO]

Shumaker, R. W., Walkup, K. R. \& Beck, B. B. (2011) Animal tool behavior. Johns Hopkins University Press. [aFO]

Silveri, M. C. \& Ciccarelli, N. (2009) Semantic memory in object use. Neuropsychologia 47:2634-41. [arFO]

Simms, N. K., Frausel, R. R. \& Richland, L. E. (2018) Working memory predicts children's analogical reasoning. Journal of Experimental Child Psychology 166:160-77. [AM-R]

Simonton, D. K. (2000) Creativity: Cognitive, personal, developmental, and social aspects. American Psychologist 55:151-58. [arFO]

Sirianni, G., Mundry, R. \& Boesch, C. (2015) When to choose which tool: Multidimensional and conditional selection of nut-cracking hammers in wild chimpanzees. Animal Behaviour 100:152-65. [aFO, CB]

Sirianni, G., Wittig, R. M., Gratton, P., Mundry, R., Schuler, A. \& Boesch, C. (2018) Do chimpanzees anticipate an object's weight? A field experiment on the kinematics of hammer-lifting movements in the nut-cracking Tai chimpanzees. Animal Cognition 21:109-18. [aFO, CB]

Sirigu, A., Duhamel, J. R. \& Poncet, M. (1991) The role of sensorimotor experience in object recognition. Brain 114:2555-73. [rFO]

Slater, P. J. B. (1983) Bird song learning: Theme and variations. Perspectives in Ornithology 12:475-99. [AHT]

Smith, C., Gabora, L. \& Gardner-O'Kearny, W. (2018) The extended evolutionary synthesis facilitates evolutionary models of culture change. Cliodynamics: The Journal of Quantitative History and Cultural Evolution 9(2):84-107. [LG]

Smulders, T., Gould, K. \& Leaver, L. (2010) Using ecology to guide the study of cognitive and neural mechanisms of different aspects of spatial memory in food-hoarding animals. Philosophical Transactions of the Royal Society B: Biological Sciences 365:883-900. [CB]

Sohlberg, M. M. \& Mateer, C. A. (2017) Cognitive rehabilitation: An integrative neuropsychological approach. Guilford Publications. [JFB]

Southgate, V. \& Csibra, G. (2009) Inferring the outcome of an ongoing novel action at 13 months. Developmental Psychology 45(6):1794-98. [MH]

Spagnoletti, N., Visalberghi, E., Ottoni, E., Izar, P. \& Fragaszy, D. (2011) Stone tool use by adult wild bearded capuchin monkeys (Cebus libidinosus). Frequency, efficiency, and tool selectivity. Journal of Human Evolution 61:97-107. [aFO]

Span, M., Hettinga, M., Vernooij-Dassen, M., Eefsting, J. \& Smits, C. (2013) Involving people with dementia in the development of supportive IT applications: A systematic review. Ageing Research Reviews 12(2):535-51. [JFB]

Spek, A. A. \& Velderman, E. (2013) Examining the relationship between autism spectrum disorders and technical professions in high functioning adults. Research in Autism Spectrum Disorders 7:606-12. Available at: https://doi.org/10.1016/j.rasd.2013.02.002. [BJC]

Sperber, D. (1996) Explaining culture: A naturalistic approach. Blackwell. [aFO]

Sperber, D. \& Hirschfeld, L. A. (2004) The cognitive foundations of cultural stability and diversity. Trends in Cognitive Sciences 8:40-46. [aFO]

Spunt, R. P. \& Lieberman, M. D. (2012) Dissociating modality-specific and supramodel neural systems for action understanding. The Journal of Neuroscience 32:3575-83. [rFO]

St Clair, J. J. H., Burns, Z. T., Bettaney, E., Morrissey, M. B., Otis, B., Ryder, B., Fleischer, R. C., James, R. \& Rutz, C. (2015) Experimental resource pulses influence socialnetwork dynamics and the potential for information flow in tool-using crows. Nature Communications 6:7197. [CR]

St Clair, J. J. H., Klump, B. C., Sugasawa, S., Higgott, C. G., Colegrave, N. \& Rutz, C. (2018) Hook innovation boosts foraging efficiency in tool-using crows. Nature Ecology and Evolution 2(3):441-44. [AHT, CR]

St Clair, J. J. H., Klump, B. C., van der Wal, J. E. M., Sugasawa, S. \& Rutz, C. (2016) Strong between-site variation in New Caledonian crows' use of hook-tool-making materials. Biological Journal of the Linnean Society 118:226-32. [CR]

St Clair, J. J. H. \& Rutz, C. (2013) New Caledonian crows attend to multiple functional properties of complex tools. Philosophical Transactions of the Royal Society B 368:20120415. [CR]
Sterelny, K. (2016) Cumulative cultural evolution and the origins of language. Biological Theory 11(3):173-86. [NW]

Storms, G., De Boeck, P., Van Mechelen, I. \& Ruts, W. (1998) Not guppies, nor goldfish, but tumble dryers, Noriega, Jesse Jackson, panties, car crashes, bird books, and Stevie Wonder. Memory \& Cognition 26(1):143-45. [LG]

Stout, D. \& Chaminade, T. (2007) The evolutionary neuroscience of tool making. Neuropsychologia 45:1091-100. [aFO]

Stout, D. \& Hecht, E. E. (2017) Evolutionary neuroscience of cumulative culture Proceedings of the National Academy of Science USA 114:7861-68. [aFO]

Stout, D., Passingham, R., Frith, C., Ape, J. \& Chaminade, T. (2011) Technology, expertise and social cognition in human evolution. European Journal of Neuroscience 33:1328-38. [aFO]

Stout, D., Rogers, M. J., Jaeggi, A. V. \& Semaw, S. (2019) Archaeology and the origins of human cumulative culture. A case study from the earliest Oldowan at Gona, Ethiopia. Current Anthropology 60:309-40. [aFO, AW, MJO]

Stuart-Fox, M. (2014) The origins of causal cognition in early hominins. Biology and Philosophy 30:247-66. [PG]

Sugasawa, S., Klump, B. C., St Clair, J. J. H. \& Rutz, C. (2017) Causes and consequences of tool shape variation in New Caledonian crows. Current Biology 27:3885-90. [CR]

Sugiyama, Y. (1994) Tool use by wild chimpanzees. Science (New York, N.Y.) 367:327. [CB]

Sugiyama, Y. \& Koman, J. (1979) Tool-using and -making behavior in wild chimpanzees at Bossou, Guinea. Primates 20:513-24. [CB]

Tabors, P. O., Roach, K. A. \& Snow, C. E. (2001) Home language and literacy environment: Final results. In: Beginning literacy with language: Young children learning at home and school, eds. D. K. Dickinson \& P. O. Tabors, pp. 111-38. Paul H Brookes Publishing. [ASH]

Talanquer, V. (2007) Explanations and teleology in chemistry education. International Journal of Science Education 29(7):853-70. [MH]

Taylor, A. H. (2014) Corvid cognition. Wiley Interdisciplinary Reviews: Cognitive Science 5(3):361-72. [AHT]

Taylor, A. H., Cheke, L. G., Waismeyer, A., Meltzoff, A. N., Miller, R., Gopnik, A., Clayton, N. S. \& Gray, R. D. (2014) Of babies and birds: Complex tool behaviours are not sufficient for the evolution of the ability to create a novel causal intervention. Proceedings of the Royal Society B: Biological Sciences 281(1787):20140837. [AHT, CR]

Taylor, A. H., Cheke, L. G., Waismeyer, A., Meltzoff, A., Miller, R., Gopnik, A., .. \& Gray, R. D. (2015) No conclusive evidence that corvids can create novel causal interventions. Proceedings of the Royal Society B: Biological Sciences 282(1813):20150796. [AHT]

Taylor, A. H., Elliffe, D. M., Hunt, G. R., Emery, N. J., Clayton, N. S. \& Gray, R. D. (2011) New Caledonian crows learn the functional properties of novel tool types. PLoS One 6 : e26887. [CR]

Taylor, A. H., Elliffe, D., Hunt, G. R. \& Gray, R. D. (2010) Complex cognition and behavioural innovation in New Caledonian crows. Proceedings of the Royal Society $B$ 277:2637-43. [CR]

Taylor, A. H., Hunt, G. R. \& Gray, R. D. (2012a) Context-dependent tool use in New Caledonian crows. Biology Letters 8(2):205-207. [AHT]

Taylor, A. H., Hunt, G. R., Holzhaider, J. C. \& Gray, R. D. (2007) Spontaneous metatool use by New Caledonian crows. Current Biology 17:1504-507. [CR, AHT]

Taylor, A. H., Hunt, G. R., Medina, F. S. \& Gray, R. D. (2009) Do New Caledonian crows solve physical problems through causal reasoning? Proceedings of the Royal Society B: Biological Sciences 276(1655):247-54. [aFO, AHT]

Taylor, A. H., Miller, R. \& Gray, R. D. (2012b) New Caledonian crows reason about hidden causal agents. Proceedings of the National Academy of Sciences of the United States of America 109(40):16389-91. [AHT, CR]

Taylor, A. H., Miller, R., Gray, R. D. (2013a) Reply to Boogert et al.: The devil is unlikely to be in association or distraction. Proceedings of the National Academy of Sciences of the United States of America 110(4):E274. [AHT]

Taylor, A. H., Miller, R., Gray, R. D. (2013b) Reply to Dymond et al.: Clear evidence of habituation counters counterbalancing. Proceedings of the National Academy of Sciences of the United States of America 110(5):E337. [AHT]

Tecwyn, E. C., Thorpe, S. K. S. \& Chappell, J. (2014) Development of planning in 4- to 10 -year-old children: Reducing inhibitory demands does not improve performance. Journal of Experimental Child Psychology 125:85-101. [ERRB]

Tenney, E. R., Meikle, N. L., Hunsaker, D., Moore, D. A. \& Anderson, C. (2019) Is overconfidence a social liability? The effect of verbal versus nonverbal expressions of confidence. Journal of Personality and Social Psychology 116:396-415. [JLT]

Tennie, C., Braun, D. R., Premo, L. S. \& McPherron, S. P. (2016a) The island test for cumulative culture in Paleolithic cultures. In: The nature of culture: Based on an interdisciplinary symposium 'the nature of culture', eds. M. N. Haidle, N. J. Conard, \& M. Bolus, pp. 121-33. Springer. [aFO]

Tennie, C., Call, J. \& Tomasello, M. (2009) Ratcheting up the ratchet: On the evolution of cumulative culture. Philosophical Transactions of the Royal Society of London B 364:2405-15. [aFO, AM-R, TG]

Tennie, C., Call, J. \& Tomasello, M. (2010) Evidence for emulation in chimpanzees in social settings using the floating peanut task. PLoS One 5:e10544. [arFO, AM-R, AW] Tennie, C., Call, J. \& Tomasello, M. (2012) Untrained chimpanzees (Pan troglodytes schweinfurthii) fail to imitate novel actions. PLoS One 7:e41548. [aFO, AM-R] 
Tennie, C., Jensen, K. \& Call, J. (2016b) The nature of prosociality in chimpanzees. Nature Communications 7:13915. [aFO]

Tennie, C., Premo, L. S., Braun, D. R. \& McPherron, S. P. (2017) Early stone tools and cultural transmission: Resetting the null hypothesis. Current Anthropology 58(5):652-72. doi: 10.1086/693846. [TG]

Thornton, A. \& Clutton-Brock, T. (2011) Social learning and the development of individual and group behaviour in mammal societies. Philosophical Transactions of the Royal Society B 366:978-87. Available at: https://doi.org/10.1098/rstb.2010.0312. [AT]

Thornton, A. \& Lukas, D. (2012) Individual variation in cognitive performance: Developmental and evolutionary perspectives. Philosophical Transactions of the Royal Society B: Biological Sciences 367:2773-83. [CB]

Thornton, A. \& McAuliffe, K. (2006) Teaching in wild meerkats. Science (New York, N.Y.) 313:227-29. [aFO]

Thornton, A. \& Raihani, N. (2008) The evolution of teaching. Animal Behaviour 75:1823-36. [aFO, AT]

Tomasello, M. (1999) The human adaptation for culture. Annual Review of Anthropology 28:509-29. [aFO]

Tomasello, M. (2016) Cultural learning redux. Child Development 87(3):643-53. [NW]

Tomasello, M. (2019a) Becoming human: A theory of ontogeny. Belknap Press. [HM]

Tomasello, M. (2019b) On becoming human: A theory of ontogeny. Harvard University Press. [CB]

Tomasello, M. \& Call, J. (1997) Primate cognition. Oxford University Press. [aFO]

Tomasello, M., Carpenter, M., Call, J., Behne, T. \& Moll, H. (2005) Understanding and sharing intentions: The origins of cultural cognition. Behavioral and Brain Sciences 28(5):675-735. [aFO, TG]

Tomasello, M., Davis-Dasilva, M., Camak, L. \& Bard, K. (1987) Observational learning of tool-use by young chimpanzees. Human Evolution 2:175-83. [aFO]

Tomasello, M., Kruger, A. C. \& Ratner, H. H. (1993) Cultural learning. Behavioral and Brain Sciences 16:495-552. [aFO, AT]

Tooby, J. \& DeVore, I. (1987) The reconstruction of hominid behavioral evolution through strategic modeling. In: The evolution of human behavior, ed. W. G. Kinzey, pp. 183-237. University of New York Press. [aFO]

Toth, N. (1985) The Oldowan reassessed: A close look at early stone artifacts. Journal of Archaeological Science 12:101-20. [rFO]

Toyoshima, M., Yamada, K., Sugita, M. \& Ichitani, Y. (2018) Social enrichment improves social recognition memory in male rats. Animal Cognition 21:345-51. [CB]

Tracy, J. L. (2016) Take pride: Why the deadliest sin holds the secret to human success. Houghton Mifflin Harcourt. [JLT]

Tracy, J. L. \& Matsumoto, D. (2008) The spontaneous expression of pride and shame: Evidence for biologically innate nonverbal displays. Proceedings of the National Academy of Sciences 105:11655-60. [JLT]

Tracy, J. L., Mercadante, E., Witkower, Z. \& Cheng, J. T. (in press) The evolution of pride and social hierarchy. Advances in Experimental Social Psychology. [JLT]

Tracy, J. L. \& Robins, R. W. (2004) Putting the self into self-conscious emotions: A theoretical model. Psychological Inquiry 15:103-25. [JLT]

Tracy, J. L. \& Robins, R. W. (2008) The nonverbal expression of pride: Evidence for crosscultural recognition. Journal of Personality and Social Psychology 94:516-30. [JLT]

Tracy, J. L., Shariff, A. F. \& Cheng, J. T. (2010) A naturalist's view of pride. Emotion Review 2:163-77. [JLT]

Tracy, J. L., Shariff, A. F., Zhao, W. \& Henrich, J. (2013) Cross-cultural evidence that the nonverbal expression of pride is an automatic status signal. Journal of Experimental Psychology: General 142:163-80. [JLT]

Turner, C. \& Flynn, E. (2016) Learning by diffusion: Using diffusion experiments and social network analysis to understand the dynamics of cultural evolution. In: Shared representations, eds. S. S. Obhi \& E. S. Cross, pp. 503-24. Cambridge University Press. Available at: https://doi.org/10.1017/CBO9781107279353.025. [BR]

Vaesen, K. (2012) The cognitive bases of human tool use. Behavioral and Brain Sciences 35:203-18. [arFO, TG]

Vaesen, K., Collard, M., Cosgrove, R. \& Roebroeks, W. (2016) Population size does not explain past changes in cultural complexity. Proceedings of the National Academy of Science USA 113:E2241-47. [aFO]

Vale, G. L., Davis, S. J., Lambeth, S. P., Schapiro, S. J. \& Whiten, A. (2017) Acquisition of a socially learned tool use sequence in chimpanzees: Implications for cumulative culture. Evolution and Human Behavior 38:635-644. [AW]

Vale, G. L., Flynn, E. G. \& Kendal, R. L. (2012) Cumulative culture and future thinking: Is mental time travel a prerequisite to cumulative cultural evolution? Learning and Motivation 43:220-30. [aFO]

Van Der Schalk, J., Bruder, M. \& Manstead, A. (2012) Regulating emotion in the context of interpersonal decisions: The role of anticipated pride and regret. Frontiers in Psychology 3. [JLT]

van Elk, M., van Schie, H. \& Bekkering, H. (2014) Action semantics: A unifying conceptual framework for the selective use of multimodal and modality-specific object knowledge. Physics of Life Reviews 11:220-50. [aFO]

van Horik, J. O. \& Emery, N. J. (2016) Transfer of physical understanding in a non-tool-using parrot. Animal Cognition 19:1195-203. [aFO]
Van Overwalle, F. \& Baetens, K. (2009) Understanding others' actions and goals by mirror and mentalizing systems: A meta-analysis. NeuroImage 48:564-84. [rFO]

Van Schaik, C. P., Deaner, R. O. \& Merrill, M. Y. (1999) The conditions for tool use in primates: Implications for the evolution of material culture. Journal of Human Evolution 36:719-41. [rFO]

Vasilyeva, N. \& Lombrozo, T. (2018) When and how children use explanations to guide generalizations. In: Proceedings of the 40th Annual Conference of the Cognitive Science Society, eds. T. T. Rogers, M. Rau, X. Zhu \& C. W. Kalish, pp. 2069-614. Cognitive Science Society. [ASH]

Veloz, T., Temkin, I. \& Gabora, L. (2012) A conceptual network-based approach to inferring the cultural evolutionary history of the Baltic psaltery. In: Proceedings of the 34th annual meeting of the cognitive science society, eds. N. Miyake, D. Peebles \& R P. Cooper, pp. 2487-92. Cognitive Science Society. [LG]

Virgo, J., Pillon, J., Navarro, J., Reynaud, E. \& Osiurak, F. (2017) Are you sure you're faster when using a cognitive tool? American Journal of Psychology 130:493-503. [aFO]

Visalberghi, E., Addessi, E., Truppa, V., Spagnoletti, N., Ottoni, E., Izar, P. \& Fragaszy, D. (2009a) Selection of effective stone tools by wild bearded capuchin monkeys. Current Biology 19:213-17. [aFO, JJL]

Visalberghi, E., Sabbatini, G., Taylor, A. H. \& Hunt, G. R. (2017) Cognitive insights from tool use in nonhuman animals. In: APA handbook of comparative psychology vol. 2: Perception, learning and cognition, ed. J. Call, pp. 673-701. American Psychological Association. [AW]

Visalberghi, E., Sirianni, G., Fragaszy, D. \& Boesch, C. (2015) Percussive tool use by Taï western chimpanzees and Fazenda Boa Vista bearded capuchin monkeys: A comparison. Philosophical transactions of the Royal Society B 370:20140351. [aFO]

Visalberghi, E., Spagnoletti, N., Ramos da Silva, E. D., Andrade, F. R. D., Ottoni, E., Izar, P. \& Fragaszy, D. (2009b) Distribution of potential suitable hammers and transport of hammer tools and nuts by wild capuchin monkeys. Primates 50:95-104. [aFO]

Voigt, B., Pauen, S. \& Bechtel-Kuehne, S. (2019) Getting the mouse out of the box: Tool innovation in preschoolers. Journal of Experimental Child Psychology 184:65-81. [ERRB]

Völter, C. J. \& Call, J. (2017) Causal and inferential reasoning in animals. In: APA handbook of comparative psychology vol. 2: Perception, learning and cognition, ed. J. Call, pp. 643-71. American Psychological Association. [AW]

von Bayern, A. M. P., Danel, S., Auersperg, A. M. I., Mioduszewska, B. \& Kacelnik, A. (2018) Compound tool construction by New Caledonian crows. Scientific Reports 8(1):15676. [AHT]

von Bayern, A. M. P., Heathcote, R. J. P., Rutz, C. \& Kacelnik, A. (2009) The role of experience in problem solving and innovative tool use in crows. Current Biology 19:1965-68. [CR]

Vonk, J. (2019) Sticks and stones: Associative learning alone? Learning \& Behavior. Available at: https://doi.org/10.3758/s13420-019-00387-4. [CR]

Vygotsky, L. S. (1978) Mind in society: Development of higher psychological processes. Harvard University Press. [arFO, ASH]

Wasielewski, H. (2014) Imitation is necessary for cumulative cultural evolution in an unfamiliar, opaque task. Human Nature 25:161-79. [aFO]

Watson, S. K., Botting, J., Whiten, A. \& van de Waal, E. (2018) Culture and selective social learning in wild and captive primates. In: Evolution of primate social cognition, eds. L. D. Di Paolo, F. Di Vincenzo \& F. De Petrillo, pp. 211-30. Springer. [MJO]

Wei, X., Jennifer, W. Y., Shattuck, P., McCracken, M. \& Blackorby, J. (2013) Science, technology, engineering, and mathematics (STEM) participation among college students with an autism spectrum disorder. Journal of Autism and Developmental Disorders 43:1539-46. Available at: https://doi.org/10.1007/s10803-012-1700-z. [BJC]

Weidman, A. C., Tracy, J. L. \& Elliot, A. J. (2016) The benefits of following your pride: Authentic pride promotes achievement. Journal of Personality 84:607-22. [JLT]

Weir, A. A. S., Chappell, J. \& Kacelnik, A. (2002) Shaping of hooks in New Caledonian crows. Science 297:981. [CR]

Wellman, H. M. \& Gelman, S. A. (1992) Cognitive development: Foundational theories of core domains. Annual Review of Psychology 43(1):337-75. [NW]

Whalley, C. I., Cutting, N. \& Beck, S. R. (2017) The effect of prior experience on children's tool innovation. Journal of Child Experimental Psychology 161:81-94. [ERRB]

Whitehead, H. \& Rendell, L. (2015) The cultural lives of whales and dolphins. University of Chicago Press. [MJO]

Whiten, A., ed. (1991) Natural theories of mind: Evolution, simulation, and development of everyday mindreading. Basil Blackwell. [aFO]

Whiten, A. (1998) Imitation of the sequential structure of actions by chimpanzees (Pan troglodytes). Journal of Comparative Psychology 112:270-81. [aFO]

Whiten, A. (2001) Tool tests challenge chimpanzees. Nature 409:133. [CB]

Whiten, A. (2015) Experimental studies illuminate the cultural transmission of percussive technologies in Homo and Pan. Philosophical Transactions of the Royal Society B 370:20140359. [aFO]

Whiten, A. (2019a) Cultural evolution in animals. Annual Review of Ecology, Evolution and Systematics 50:1-22. Available at: https://doi.org/10.1146/annurev-ecolsys110218-025040. [AT]

Whiten, A. (2019b) Culture in the stone age. Current Anthropology 60:333-34. [aFO] 
Whiten, A. (2020) Wild chimpanzees scaffold youngsters' learning in a high-tech community. Proceedings of the National Academy of Sciences 117(2):802-804. Available at: https://doi.org/10.1073/pnas.1920430117. [BJC]

Whiten, A. \& Erdal, D. (2012) The human socio-cognitive niche and its evolutionary origins. Philosophical Transactions of the Royal Society B 367:2119-29. [aFO]

Whiten, A., Goodall, J., McGrew, W. C., Nishida, T., Reynolds, V., Sugiyama, Y., Tutin, C. E. G., Wrangham, R. W. \& Boesch, C. (1999) Cultures in chimpanzees. Nature 399:682-85. [aFO]

Whiten, A. \& Ham, R. (1992) On the nature and evolution of imitation in the animal kingdom: Reappraisal of a century of research. Advances in the Study of Behavior 21:239-83. [aFO]

Whiten, A., Horner, V. \& de Waal, F. B. M. (2005) Conformity to cultural norms of tool use in chimpanzees. Nature 437:737-40. [aFO, AM-R]

Whiten, A., Horner, V. \& Marshall-Pescini, S. R. J. (2003) Cultural panthropology. Evolutionary Anthropolology 12:92-105. [aFO, AW]

Whiten, A., McGuigan, H., Marshall-Pescini, S. \& Hopper, L. M. (2009) Imitation, overimitation, emulation and the scope of culture for child and chimpanzee. Philosophical Transactions of the Royal Society B 364:2417-28. [aFO, AW]

Whiten, A., Spiteri, A., Horner, V., Bonnie, K. E., Lambeth, S. P., Schapiro, S. J. \& de Waal, F. B. M. (2007) Transmission of multiple traditions within and between chimpanzee groups. Current Biology 17:1038-43. [aFO]

Whiten, A. \& van de Waal, E. (2017) Social learning, culture and the 'socio-cultural brain' of human and non-human primates. Neuroscience \& Biobehavioral Reviews 82:58-75. [aFO, MJO]

Whiten, A. \& van Schaik, C. (2007) The evolution of animal 'cultures' and social intelligence. Philosophical Transactions of the Royal Society B: Biological Sciences 362(1480):603. [NC]

Willard, A. K., Busch, J. T. A., Cullum, K. A., Letourneau, S. M., Sobel, D. M., Callanan, M. \& Legare, C. H. (2019) Explain this, explore that: A study of parent-child interaction in a children's museum. Child Development 90(5):e598-e617. Available at: https://doi.org/ 10.1111/cdev.13232. [ASH]

Willey, G. R. \& Phillips, P. (1958) Method and theory in American archaeology. University of Chicago Press. [MJO]

Williams, L. A. \& DeSteno, D. (2008) Pride and perseverance: The motivational role of pride. Journal of Personality and Social Psychology 94:1007-1017. [JLT]

Wimpenny, J. H., Weir, A. A. S., Clayton, L., Rutz, C. \& Kacelnik, A. (2009) Cognitive processes associated with sequential tool use in New Caledonian crows. PLoS One 4:e6471. [CR]
Wimpenny, J. H., Weir, A. A. \& Kacelnik, A. (2011) New Caledonian crows use tools for non-foraging activities. Animal Cognition 14(3):459-64. [AHT]

Windham, G. C., Fessel, K. \& Grether, J. K. (2009) Autism spectrum disorders in relation to parental occupation in technical fields. Autism Research 2:183-91. Available at: https://doi.org/10.1002/aur.84. [BJC]

Witkower, Z., Tracy, J. L., Cheng, J. T. \& Henrich, J. (2019) Two signals of social rank: Prestige and dominance are associated with distinct nonverbal displays. Journal of Personality and Social Psychology. [JLT]

Wohlschläger, A., Gattis, M. \& Bekkering, H. (2003) Action generation and action perception in imitation: An instance of the ideomotor principle. Philosophical Transactions of the Royal Society B 358:501-15. [aFO]

Wolpert, L. (2003) Causal belief and the origins of technology. Philosophical Transactions of the Royal Society A 361:1709-19. [aFO]

Wozniak, S. (2007) Iwoz: Computer geek to cult icon. WW Norton \& Company. [BJC]

Wu, Y.-H., Lewis, M. \& Rigaud, A.-S. (2019) Cognitive function and digital device use in older adults attending a memory clinic. Gerontology and Geriatric Medicine 5. [JFB]

Wynn, T. (1993) Layers of thinking in tool behavior. In: Tools, language and cognition in human evolution, eds. K. R. Gibson \& T. Ingold, pp. 389-406. Cambridge University Press. [aFO]

Wynn, T. \& Coolidge, F. L. (2007) Did a small but significant enhancement in workingmemory capacity power the evolution of modern thinking? In: Rethinking the human revolution: New behavioural and biological perspectives on the origin and dispersal of modern humans, eds. P. Mellars, K. Boyle, O. Bar-Yosef \& C. Stringer, pp. 79-90. McDonald Institute Monographs. [aFO, AM-R]

Wynn, T. \& Coolidge, F. L. (2014) Technical cognition, working memory and creativity. Pragmatics \& Cognition 22:45-63. [arFO, AM-R]

Wynn, T., Haidle, M. N., Lombard, M. \& Coolidge, F. L. (2017) The expert cognition model in human evolutionary studies. In: Cognitive models in paleolithic archaeology, eds. T. Wynn \& F. L. Coolidge, pp. 21-44. Oxford University Press. [aFO]

Yellen, J. E., Brooks, A. S., Cornelissen, E., Mehlman, M. J. \& Stewart, K. (1995) A middle stone age worked bone industry from Katanda, Upper Semliki Valley, Zaire. Science (New York, N.Y.) 268(5210):553-56. [AHT]

Zilhāo, J. (2019) Commentary. Tar adhesives, Neanderthals, and the tyranny of the discontinuous mind. Proceedings of the National Academy of Sciences. Available at: https://doi.org/10.1073/pnas.1916116116. [PG]

Zwirner, E. \& Thornton, A. (2015) Cognitive requirements of cumulative culture: Teaching is useful but not essential. Scientific Reports 5:16781. Available at: https:// doi.org/10.1038/srep16781. [aFO, AT] 